\title{
MECHANISMS OF IUMMUNITY AND INFLAMMATION IN BRUCELLA-INDUCED ARTHRITIS
}

\author{
A Dissertation \\ Presented to \\ The Faculty of the Graduate School \\ at the \\ UNIVERSITY OF MISSOURI \\ In Partial Fulfillment \\ of the Requirements for the Degree \\ Doctor of Philosophy \\ in Microbiology
}

By

CAROLYN ANN LACEY

Dr. Jerod Alan Skyberg, Dissertation Advisor

May 2018 
The undersigned, appointed by the dean of the Graduate School, have examined the dissertation entitled:

\section{MECHANISMS OF IMMUNITY AND INFLAMMATION IN BRUCELLA-INDUCED ARTHRITIS}

Presented by Carolyn A. Lacey,

a candidate for the degree of doctor of philosophy,

and hereby certify that, in their opinion, is worthy of acceptance,

Certified

by

Jerod A. Skyberg

Assistant Professor of Veterinary Pathology

Accepted

by

Deborah M. Anderson

Associate Professor of Veterinary Pathology

Accepted

by

Charles R. Brown

Professor of Veterinary Pathology

Accepted

by

Sheila A. Grant

Professor of Bioengineering

Accepted

by

Craig L. Franklin

Professor of Veterinary Pathology 


\section{DEDICATION}

To Mom and Dad and Grandma and Grandpa Lacey, who fostered my curiosity and creativity as a child so I could become a creative and curious adult. 


\section{ACKNOWLEDGEMENTS}

I am grateful to all of those with whom I have had the pleasure to work with during these and other related projects. Assistance provided by the excellent staff at the University of Missouri, Laboratory for Infectious Disease Research was greatly appreciated. My special thanks are extended to Travis McCarthy for his constant support when I worked many long days in the BSL-3, and for always being willing to help when I had to radio out of the lab when I forgot something.

I would also like to express my gratitude to Dr. William Mitchell who scored the histology slides seen in the works here, and without whose work would not have made this dissertation possible. Thanks for teaching me about joint histology and answering all of my many questions.

I would like to acknowledge each of the members of my Dissertation Committee, Dr. Deborah Anderson, Charles Brown, Sheila Grant and Craig Franklin, as each of them has guided me to succeed in these scientific endeavors, and has provided me with a great deal of advice and encouragement. I would like to give a special thanks to Dr. Charles Brown for being there in moments of crisis and always being full of career and life advice. Thanks for encouraging me to "run with the big dogs." You certainly kept my ambitious spirit alive through this process.

I would also like to thank my fellow graduate students in Connaway Hall. Without everyone's commitment to scientific learning we would not have developed such a strong group. Thanks for sharing ideas, reagents and most of all friendship. A special thanks to Carrie Lasky (Williams) for helping me get 
adapted to graduate school and encouraging me to do a lab rotation in Connaway Hall. Additionally, I would like to thank Alexis Dadelahi for her edits and comments during my comprehensive exam process and throughout this dissertation.

Finally, I would like to express my very great appreciation to my advisor and mentor Dr. Jerod Skyberg for taking a chance on me as a graduate student. I look up to you more than you know. This journey has challenged me further than I ever thought imaginable, and I am glad I had you as an advisor guiding me through the process. Thank you for your time in teaching me how to use the BSL-3 lab, editing several of my manuscripts, exposing me to the grant writing process and the review process, and helping me to prepare several formal presentations. I feel more than prepared for my next scientific journey because of you. 


\section{TABLE OF CONTENTS}

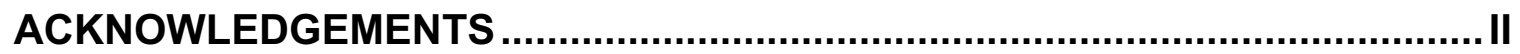

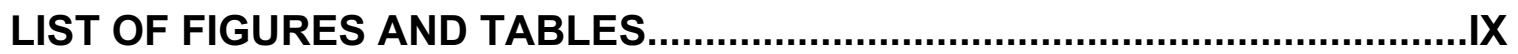

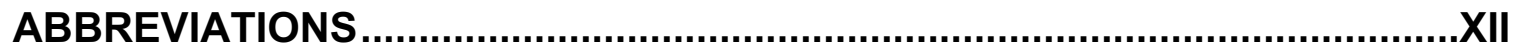

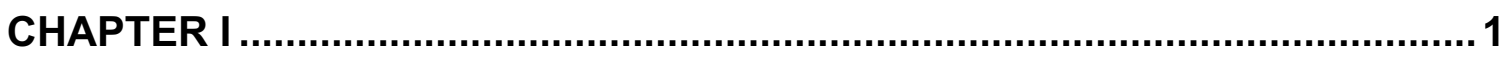

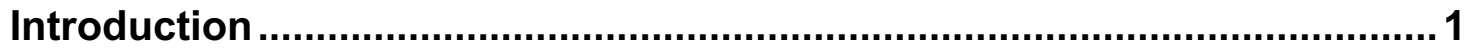

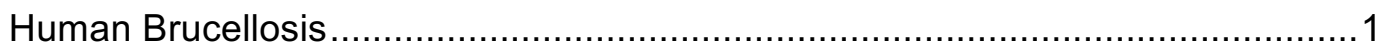

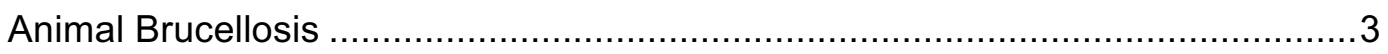

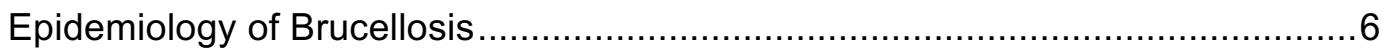

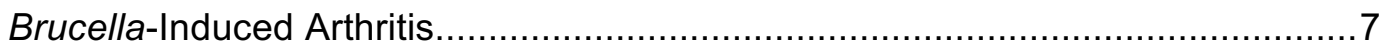

Models of Brucellosis and Brucella Induced Arthritis ..................................... 8

Inflammation and the Inflammatory Response to Brucella .................................

Brucella's Evasive Intracellular Life Cycle .................................................13

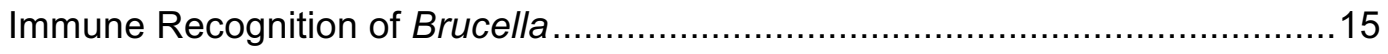

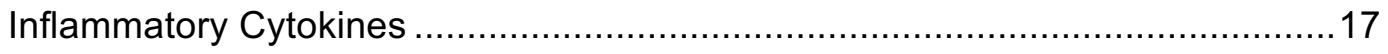

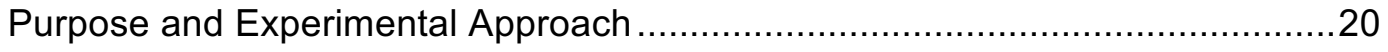

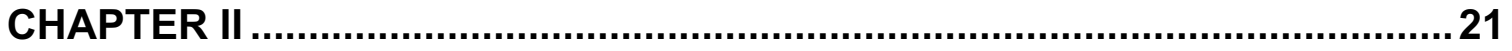

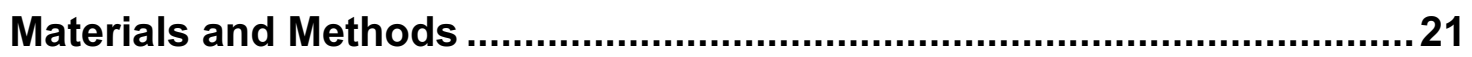

Bacterial strains and growth conditions ……..............................................21

Deletion and complementation of the B. melitensis L-lactate dehydrogenase

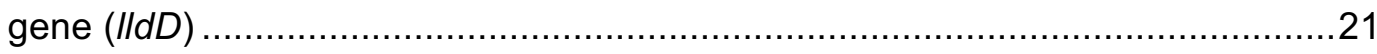

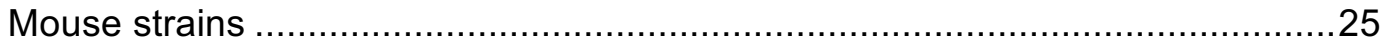

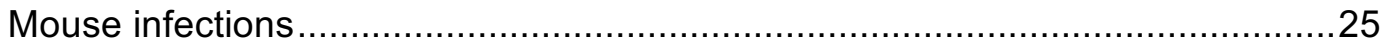




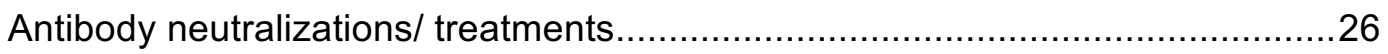

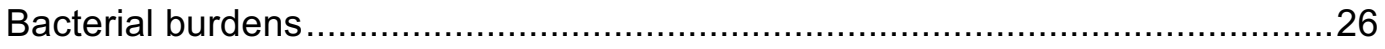

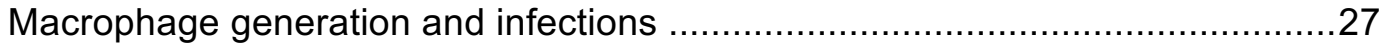

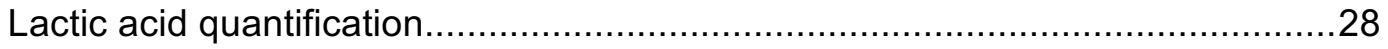

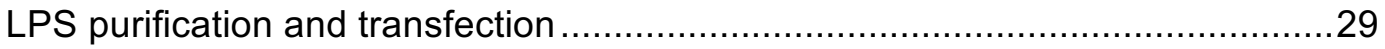

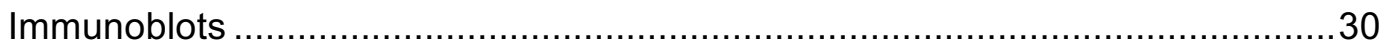

Extraction of RNA and RT-PCR analysis of joint homogenates .........................30

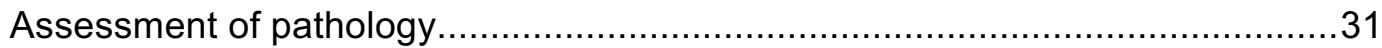

Ankle processing for cytokine measurements ...............................................34

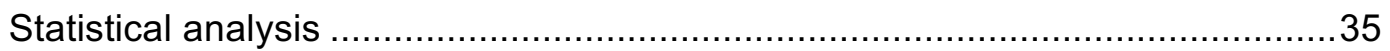

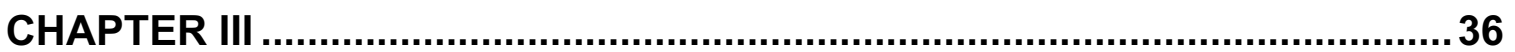

CXCR2 Mediates Brucella-Induced Arthritis in IFN-Y- Deficient Mice ${ }^{A}$......36

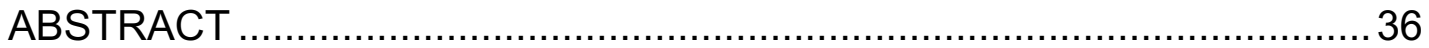

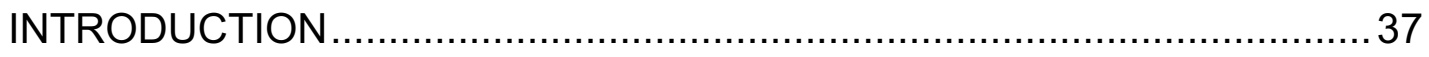

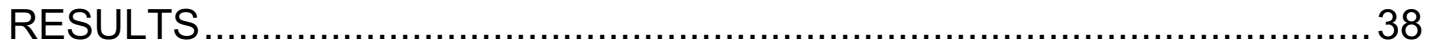

Inflamed B. melitensis-infected joints have increased levels of neutrophils ........38

T and B cells are not required for Brucella-induced focal inflammation ..............40

CCR2 and CXCR2 ligands are up-regulated in inflamed, B. melitensis-infected

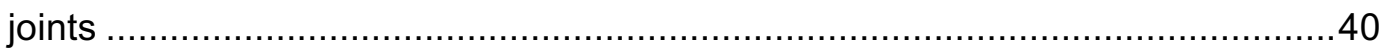

CCR2 is not a critical mediator of Brucella-induced focal inflammation ..............42

$\mathrm{CXCR2}^{-/-}$mice display reduced clinical signs of Brucella-induced inflammation .42 CXCR2 mediates neutrophil infiltration in musculoskeletal tissue ......................45

Reduced TNF- $\alpha$ and CCL3 levels in IFN- $\gamma^{-1 /} / C_{X C R 2}{ }^{-1}$ mice .............................48

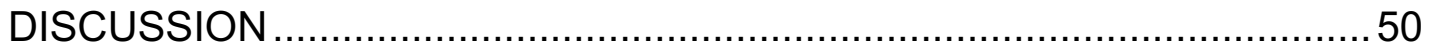

CHAPTER IV 


\section{Temporal Role for MyD88 in a Model of Brucella-Induced Arthritis and}

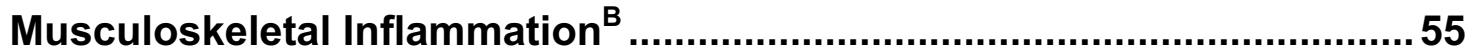

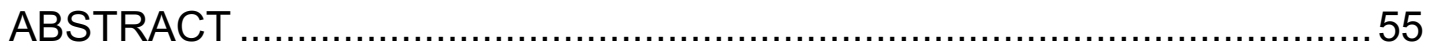

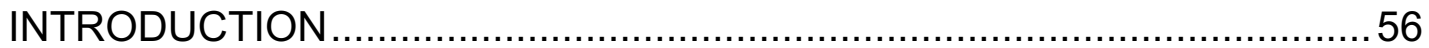

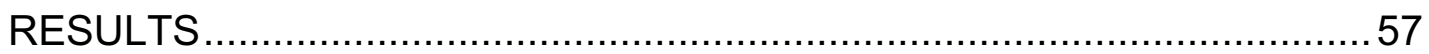

Brucella infection of the footpad results in arthritis and musculoskeletal

inflammation in wild-type mice.

Footpad inoculation of mice with $B$. melitensis, $B$. suis or $B$. abortus results in dose dependent joint swelling, arthritis and musculoskeletal inflammation.

Adaptive immune responses mediate resolution of inflammation, but are not required for development of joint swelling.

MyD88 initiates early joint swelling, but is also required for clearance of Brucella

from the joint and resolution of joint swelling

MyD88 initiates early recruitment of inflammatory infiltrates following $B$.

melitensis footpad infection.

MyD88 mediates inflammatory cytokine production in B. melitensis-infected mouse joints early in infection

MyD88 aids in systemic Brucella clearance and T cell production of IFN-Y

DISCUSSION

CHAPTER V.

Inflammasomes confer protection via IL-18 and pyroptosis, and are negatively regulated by IFN-Y-dependent nitric oxide during Brucella infection $^{\mathrm{C}}$ 


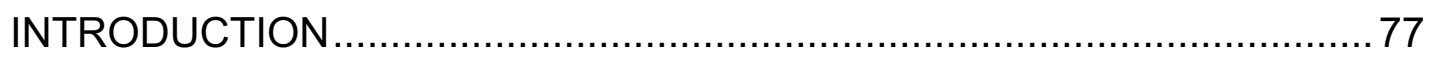

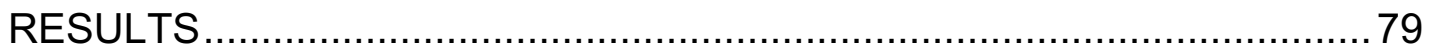

Inflammasomes have IL-1-independent effects on inflammation and control of

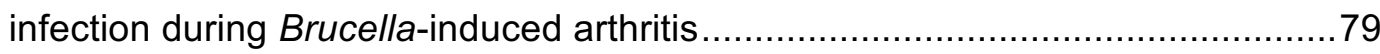

Caspase-11 contributes to Brucella-induced arthritis and restricts Brucella

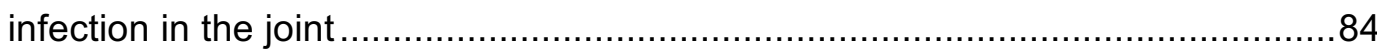

Caspase1/11-dependent IL-18 mediates joint swelling and Brucella clearance

from the joint.

Eicosanoids and perforin-mediated cytotoxicity are dispensable for control of

Brucella infection in the joint

Caspase-1 and caspase-11 induce cell death in response to Brucella and restrict infection in macrophages.

.91

Inflammasomes do not restrain Brucella infection due to their ability to restrict

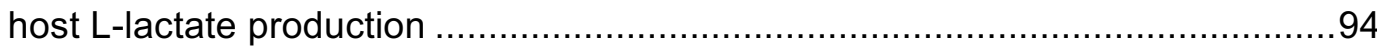

IFN- $\gamma$ restrains Caspase-1/11-induced joint inflammation .............................96

Adaptive immune cell IFN- $\gamma$ production contributes to Brucella clearance ........103

NLRP3 and Caspase-11 are partially responsible for inducing inflammation in

IFN- $\gamma$ deficient mice.

IFN- $\gamma$ induces the upregulation of iNOS, and iNOS deficiency enhances joint

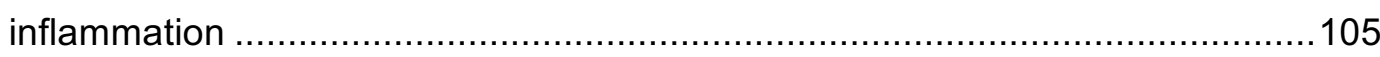

Nitric oxide inhibits Brucella-induced caspase -1 activation ..........................108

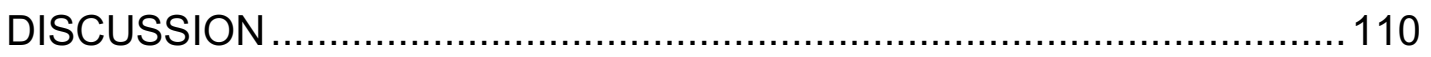

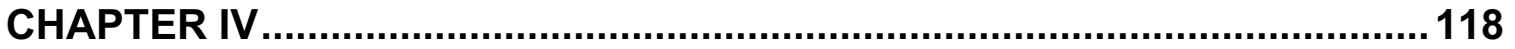

Discussion ......................................................................................... 118 
VITA 168

\section{Footnotes:}

A. Chapter II. This work was published as in the Journal of Infectious Diseases in 2016 with Carolyn A. Lacey as the first author, followed in authorship order by Lauren L. Keleher, William J. Mitchell, Charles R. Brown, and Jerod A. Skyberg. CAL conceived the project, designed, performed, analyzed experiments, and wrote the paper. LLK assisted with experiments. WJM scored histopathology. CRB analyzed experiments, and edited the paper. JAS conceived the project, designed, performed, and assisted with experiments, and edited the paper.

B. Chapter III. This work was published as in Infection and Immunity in 2017 with Carolyn A. Lacey as the first author, followed in authorship order by William J. Mitchell, Charles R. Brown, and Jerod A. Skyberg. CAL conceived the project, designed, performed, analyzed experiments, and wrote the paper. WJM scored histopathology. CRB analyzed experiments, and edited the paper. JAS conceived the project, designed, performed, and assisted with experiments, and edited the paper.

C. Chapter IV. This work was submitted for publication in 2018 with Carolyn A. Lacey as the first author, followed in authorship order by William J. Mitchell, Alexis S. Dadelahi, and Jerod A. Skyberg. CAL conceived the project, designed, performed, analyzed experiments, and wrote the paper. WJM scored histopathology. ASD provided reagents. JAS conceived the project, designed, performed, and assisted with experiments, and edited the paper. 


\section{LIST OF FIGURES AND TABLES}

\section{FIGURES}

Figure 1. T and B cells are not required for Brucella-induced focal inflammation.

Figure 2. CCR2 and CXCR2 ligands are up-regulated in inflamed, B. melitensisinfected joints.

Figure 3. CCR2 is not a critical mediator of Brucella-induced focal inflammation.

Figure 4. Reduced focal inflammation in Brucella- infected, anti-IFN- $\gamma$-treated, CXCR2 $2^{-/-}$

mice

Figure 5. CXCR2 mediates Brucella-induced inflammation in IFN- $\gamma^{-/-}$mice.

Figure 6. CXCR2 mediates musculoskeletal neutrophil recruitment in Brucella-infected

mice.

Figure 7. Reduced levels of TNF- $\alpha$ and CCL3 in IFN- $\gamma^{-/-}$mice lacking CXCR2.

Figure 8. Model of Brucella Induced Arthritis in i.p. infected IFN-ץ deficient mice.

Figure 9. WT mice develop arthritis and musculoskeletal inflammation following footpad

infection

Figure 10. WT mice infected via the footpad with $B$. melitensis, $B$. abortus or $B$. suis develop arthritis and musculoskeletal inflammation.

Figure 11. Adaptive immune responses are needed for inflammation resolution but not required for inflammation onset.

Figure 12. MyD88 ${ }^{-/-}$mice have delayed joint swelling but increased joint Brucella burden. 65

Figure 13. MyD88 mediates early arthritis and musculoskeletal inflammation 67 
Figure 14. MyD88 ${ }^{-/}$joints have delayed cytokine production following footpad infection with $B$. 68

Figure 15. MyD88 aids in systemic Brucella clearance and T cell IFN-y production ... 70

Figure 16. Caspase-1/11 induces inflammation and controls joint Brucella burden ....80

Figure 17. Caspase1/11 deficiency results in delayed resolution of joint swelling.......82

Figure 18. Inflammasomes mediate pro-inflammatory cytokine production in Brucellainfected joints

Figure 19. Caspase-11 is inflammatory and limits Brucella infection in the joints. 85

Figure 20. Caspase-11 contributes to inflammasome dependent pro-inflammatory cytokine production in Brucella-infected joints

Figure 21. IL-18 contributes to inflammasome dependent joint inflammation and Brucella clearance.

Figure 22. Eicosanoids and perforin are dispensable for control of Brucella infection in the joint

Figure 23. Brucella induces caspase-1 and caspase-11 dependent pyroptosis.

Figure 24. Brucella infection induces caspase-1/11-dependent cell death in vivo, and transfection of Brucella LPS induces caspase-11 dependent cell death in vitro

Figure 25. Increased lactate production by Caspase- $1 / 11^{-/}$macrophages does not promote Brucella infection

Figure 26. 2-DG prevents swelling during Brucella infection.

Figure 27. IL-18 contributes to IFN-y production in Brucella infected joints.

Figure 28. IFN-y deficiency results in severe caspase-1/11-dependent inflammation 
Figure 29. IFN-y deficiency leads to increased myeloid cell recruitment in Brucella infected joints

Figure 30. IFN-y deficiency leads to increased inflammasome dependent IL-1 $\beta$ in vivo.

Figure 31. Adaptive immune cell IFN- $\gamma$ production is important for Brucella clearance.

Figure 32. NLRP3 and Caspase-11 induce inflammation in the absence of IFN- $\gamma \ldots 106$

Figure 33. Nitric oxide inhibits Brucella-induced caspase-1 activation and IL-1 $\beta$

production.

Figure 34. In vivo inhibition of iNOS with AGHS results in enhanced joint inflammation

Figure 35. Possible mechanisms of inflammation during Brucella induced arthritis. . 140

\section{TABLES}

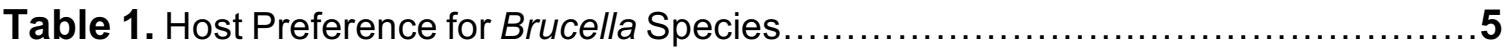

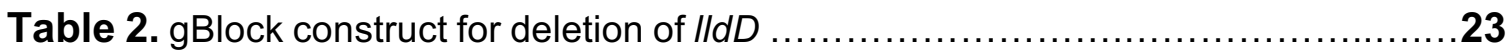

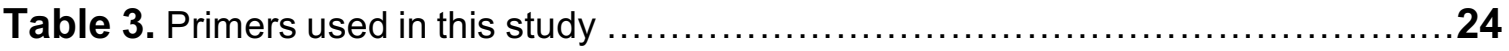

Table 4. Advantages of the murine models of Brucella-induced arthritis...............120 


\section{ABBREVIATIONS}

2-DG

A AAMs

AGHS

AIM2

ASC

B B.a.

B.m.

B.s.

$\mathrm{Ba}$

BCA

BCV

BMDMs

BSL-3

C CAMs

Casp1

Casp11

CDC

cDNA

CFUs

CM

CPG DNA

D DAMPS

DNA

E ELISA

ER

F f.p.

FACs

FBS

FLS
2-Deoxy-glucose

alternatively activated macrophages

aminoguanidine hemisulfate salt

absent in melanoma 2

apoptosis-associated speck like protein

Brucella abortus

Brucella melatinsis

Brucella suis

Brucella agar

bicinchoninic acid assay

Brucella containing vacuole

bone marrow derived Macrophages

biosafety Level-3

classically activated macrophages

caspase-1

caspase-11

Centers for Disease Control and Prevention

complementary deoxyribonucleic acid

colony-forming units

Complete Media

cytidine-phosphateguanosine deoxyribonucleic acid

damage associated molecular patterns

deoxyribonucleic acid

enzyme-linked immunosorbent assay

endoplasmic reticulum

footpad

fluorescence-activated cells sorting

fetal bovine serum

fibroblast-like synoviocytes 


$\begin{array}{lll}\text { G } & \text { GAPDH } & \text { glyceraldehyde 3-phosphate dehydrogenase } \\ & \text { GBP } & \text { guanylate binding proteins } \\ \text { H } & \text { H\&E } & \text { Haemotoxylin and Eosin } \\ & \text { HMGB1 } & \text { high mobility group box 1 } \\ & \text { i.p. } & \text { intraperitoneal } \\ & \text { IFN } & \text { interferon } \\ & \text { IFNAR } & \text { interferon alpha/beta receptor } \\ & \text { IHC } & \text { immunohistochemistry } \\ & \text { IL-18R } & \text { interleukin-18 receptor } \\ & \text { IL-18RA } & \text { interleukin-18 receptor antagonist } \\ & \text { IL-1R } & \text { interleukin-1 receptor } \\ & \text { ILCs } & \text { innate lymphoid cells } \\ \text { iNOS } & \text { inducible nitric oxide synthase } \\ \text { K } & \text { KanR } & \text { kanamycin resistance } \\ \text { L } & \text { LAMP1 } & \text { lysosome-associated membrane protein } \\ & \text { LDH } & \text { lactate dehydrogenase } \\ \text { IIdD } & \text { lactate dehydrogenase (gene) } \\ \text { LPS } & \text { lipopolysaccharide }\end{array}$

M M-CSF macrophage colony-stimulating factor

MMPs matrix metalloproteinase

mRNA messenger ribonucleic acid

MWCO molecular weight cut-off

MyD88 myeloid differentiation factor 88

N NETs neutrophil extracellular traps

NK cells natural killer cells

NLRP3 NACHT, LLR and PYD domains-containing protein 3

NLRs nucleotide-binding oligomerization domain-like receptors

NO nitric oxide

NOS2 nitric oxide synthase 2 (inducible)

O OD optical density

P PAMPs pathogen associated molecular patterns 
PBS

PCR

Perf

PMN

PRRs

R Rag1

RNA

ROS

RPMI medium

S SD

SEM

SNAP

SPBS

spp.

SYK

T T4SS

TAK1

TcpB

TLR

TNFR

TRIF

TUNEL

V VLCFAs

W WT

T TCRa phosphate buffered saline

polymerase chain reaction

perforin

polymorphonuclear

pathogen recognition receptors

recombination activating gene 1

ribonucleic acid

reactive oxygen species

Roswell Park Memorial Institute medium

standard deviation

standard error of the mean

S-nitroso-N-acetyl-penicillamine

sterile phosphate buffered saline

species

spleen tyrosine kinase

type IV secretion system

transforming growth factor- $\beta$-activated kinase-1

Brucella TIR-domain-containing protein

Toll-like receptor

tumor necrosis factor receptor

TIR domain-containing adaptor-inducing inferferron- $\beta$

terminal deoxynucleotidyl transferase nick-end

labeling

very long chain fatty acid

wildtype

T-cell receptor alpha chain 


\section{CHAPTER I}

\section{Introduction}

\section{Human Brucellosis}

The genus Brucella is a group of Gram-negative facultative intracellular cocobacilli that cause the zoonotic disease brucellosis. Human brucellosis is a systemic disease often referred to as undulant fever due to the characteristic interspersed fevers often displayed in infected patients (2). Brucella infects phagocytic cells of the reticuloendothelial system, particularly macrophages, as well as placental trophoblasts in pregnant animals (3). Clinical presentation in humans varies but generally consists of flu like symptoms such as malaise, chills and headaches, and, in acute forms, hepatomegaly and splenomegaly $(4,5)$. Disease manifestations typically present between 5 and 60 days post infection but brucellosis can also be asymptomatic in humans $(6,7)$. Brucella can infect almost any tissue and cause disease with relapse and lifelong complications, including arthritis, liver abscesses, and possible neurological disorders. While generally not life-threatening Brucella infection can cause endocarditis, which is a significant contributor to the $5 \%$ fatality rate associated with disease $(1,4,8,9)$. The most prevalent species infectious to humans are $B$. melitensis, $B$. abortus and $B$. suis which have preferred domestic animal reservoirs, but are not limited to, ruminants, bovine and swine, respectively $(10,11) . B$. melitensis is the most virulent species in humans and is most frequently reported as the cause of human disease (12). The genome of Brucella spp. are very similar to one another, exhibiting greater 
than $99 \%$ amino acid identity between orthologs (13). Brucella's genetic conservation and diverse range of hosts indicates these organisms are highly evolved for survival within mammals, although it remains unclear why there is such great diversity in host preference $(14,15)$.

Transmission to humans typically occurs through the consumption of unpasteurized milk or aerosol following contact with contaminated meat products, but may also spread through skin abrasions $(16,17)$. Brucella spp. infect a variety of wild and domestic livestock providing occupational hazards for veterinarians, meat processors and other individuals repeatedly exposed to animals $(7,17,18)$. Brucellosis seldom spreads from person to person, and over $50 \%$ of human-tohuman transfer cases are in patients less than one year old, indicating dissemination due to breastfeeding or vertical transmission. Rare cases of disease passage between humans through blood transfusions, bone marrow transplants and sexual transmission have also been reported. $(12,19,20)$. Brucellosis is also highly contagious with an infectious dose as low as 10 bacteria, making brucellosis the top laboratory acquired infection in the world (21).

Due to Brucella's ability to easily infect humans through aerosol and the potential to exhaust healthcare and agricultural resources, Brucella poses a major threat to public health and safety. Weaponizing Brucella may be of potential interest to terrorist organizations, thus Brucella has been designated a potential biological warfare threat. In fact, Brucella was the first biological agent to be weaponized by the United States, and the former Soviet Union tested Brucella in their offensive biological weapons program $(22,23)$. Thus, Brucella spp. have 
been named non-Tier 1 select agents by the Centers for Disease Control (CDC) (24).

There are currently no human vaccines licensed to prevent human bucellosis and diagnosis can be difficult. The gold standard for Brucella diagnostics is through culture, however blood samples are often false negatives and growth is slow. Thus, serology is often used in conjunction with culture for rapid and accurate diagnosis (25). Uncomplicated cases of brucellosis are generally treated with a rigorous course of antibiotics, typically 6 weeks of doxycycline in combination with 2-3 weeks of an aminoglycoside (such as streptomycin or gentamycin), or 6 weeks of rifampin $(26,27)$. Despite antibiotic therapy resistant abscesses may still persist, and relapse of infection is common leading to chronic arthritis and endocarditis (5, 28, 29). Additionally, Brucella DNA can be detected in the patient's blood long after clinical manifestations have subsided, and some patients never clear infection which can lead to lifelong complications (28).

\section{Animal Brucellosis}

Brucella spp. are highly adapted to surviving in a variety of hosts. B. canis which preferentially infects dogs can cause brucellosis in humans; however, transmission is only mild when compared with other Brucella spp. Additionally, there are other species within the genus Brucella that have modest to no reported human transmission cases, including B. ovis, B. neotomae and B. microti (30). In fact, some species even infect marine mammals. B. ceti has been isolated in cetaceans such as dolphins, whales and porpoises and B. pinnipediae infects 
pinnipeds which include sea lions and seals. Although rare, there is evidence that these marine species can also infect humans (Table 1) (31, 32). Despite Brucella's diverse host tropism, agricultural animals are the main source of human infections. Brucellosis incidence in many countries has been reduced due to the use of liveattenuated vaccines in livestock; these consist of $B$. abortus vaccines, S19 and RB51 and B. melitensis vaccine Rev1. Although vaccination of animals reduces human disease incidence (33), these vaccines often present risks for veterinarians and animal caretakers as they cause disease in humans upon incidental exposure (34-36).

There is also risk for reintroduction of brucellosis to livestock as free-range wildlife can carry the disease. Populations of bison and elk often harbor $B$. abortus in the greater Yellowstone area, some feral swine in the southern United States test positive for $B$. suis and Alaskan caribou and reindeer also exhibit brucellosis positive titers $(37,38)$. Although brucellosis in animals is not associated with a unique set of symptoms, disease can present as mastitis, lameness and lymphadenitis (39). Additionally, there are rare cases of $B$. melitensis infected goats and sheep developing arthritis, infected horses can display local abscesses in bursae and dogs with $B$. canis can develop discospondylitis $(40,41)$ Nonetheless, the best characterized manifestations associated with animal brucellosis are placentitis followed by spontaneous abortion in pregnant females, generally in the $3^{\text {rd }}$ trimester, and epididymitis and/or orchitis, which can lead to sterility in males $(25,40)$. Due to the abundance of brucellae in aborted fetal material, contact with birthing discharge or an infected fetus and/or placenta 


\begin{tabular}{|c|c|c|}
\hline Species & Zoonotic Potential & Host Preference \\
\hline Brucella melitensis & High & Sheep, Goat \\
\hline Brucella abortus & Moderate & Cattle, Bison, Elk \\
\hline Brucella suis & Moderate & Pig \\
\hline Brucella canis & Mild & Dog \\
\hline Brucella ovis & Absent & Sheep \\
\hline Brucella neotomae & Absent & $\begin{array}{c}\text { Desert wood rat } \\
\text { (Neotomae lepida) }\end{array}$ \\
\hline Brucella ceti & Mild & $\begin{array}{c}\text { Whales, Dolphins, } \\
\text { Porpoises }\end{array}$ \\
\hline Brucella pinnipedialis & Mild & Sealions, Seals \\
\hline Brucella microti & Absent & Common voles (microtus \\
\hline
\end{tabular}

Table 1. Host Preference for Brucella Species. Brucella species and their potential to infect/cause human disease (zoonotic potential), and the preferential host reservoir associated with each species. Adapted from Xavier et al, 2010.(1) 
remains the major source of natural host transmission, but animal to animal spread of disease can also occur through sex and consumption of infected milk or food (1).

\section{Epidemiology of Brucellosis}

With over 500,000 new cases annually, brucellosis is one of the most common zoonotic diseases worldwide, and has recently been designated as a neglected zoonotic disease by the World Health Organization (2, 42). Brucellosis remains an important global health issue, as it is endemic in the Mediterranean rim, Middle East, Central Asia, South America, and Africa and in some regions disease incidence exceeds 1,000 cases per year per 1 million inhabitants $(8,10$, $43,44)$. Frequency of disease has been drastically reduced in the United States and other countries with strong public and veterinary health programs (45). These policies often exercise vaccination laws, test-and-slaughter methods and surveillance programs to identify infected animals $(33,46)$. Elevated prevalence of brucellosis is generally associated with lower income countries which lack access to proper veterinary care and surveillance as well as compensatory policies for dealing with infected livestock. Additionally, endemic brucellosis represents a major economic destabilizer as the financial burden of infected animals affects the health, nutrition, and financial livelihood of many farmers in these regions (47).

The nonspecific presentation of brucellosis results in widespread misdiagnosis and underreporting of the disease, particularly in malaria-endemic areas where similarity in disease presentation results in infections often being ascribed to malaria (10). Delays in health-seeking behavior, variable performance 
of serological tests and the lack of sufficient knowledge about the disease amongst health care staff in endemic areas also confounds diagnosis (10). Brucellosis in the United States is considered an imported disease with most cases arising from recent trips to Mexico $(2,8,43,48)$; however, several wild animal reservoirs remain in the United Sates and many regions around the world (38). Furthermore, brucellosis has been described as a re-emerging zoonosis and there are current social misconceptions about the nutritive quality of pasteurized milk (49-52). This has led to an increase in unpasteurized dairy consumption, potentially putting more individuals at risk for contracting zoonotic diseases (50-52).

\section{Brucella-Induced Arthritis}

Joint inflammation in humans is observed upon infection with all the Brucella species that typically infect humans (53-55). Osteoarticular and musculoskeletal inflammation are the most common focal complications of human brucellosis and occur in $40-80 \%$ of Brucella-infected patients $(56,57)$. Arthritis is thought to arise from the hematogenous spread of Brucella to the joints, as viable brucellae can be found within the synovial fluid of infected patients $(58,59)$. Arthritis and osteoarticular complications can manifest as peripheral arthritis, sacroiliitis (inflammation of the lower spine), and spondylitis (inflammation of the vertebrae), which are most common in children, young adults and older adults, respectively $(56,57,60,61)$. Inflammation of the joint synovium is prevalent in infected individuals, with polymorphonuclear and mononuclear leukocytes found in and around synovial tissue (62). Brucella-induced arthritis is commonly treated with antibiotics; however, resolution of arthritis is often protracted and the disease can 
relapse $(57,63)$. Untreated articular brucellosis can result in synovial rupture, bone destruction, cartilage degradation osteopenia and/or bursitis with the potential to cause permanent disability $(61,64-66)$. Additionally, articular brucellosis cases refractive to antibiotic treatment may require arthroscopic debridement and/or drainage of the joint (67). Bone erosion is often rapid and radiographic changes can be difficult to identify, thus individuals are typically diagnosed via radiological examination, MRI or bone scan, but may need surgery for proper diagnosis (68, $69)$.

\section{Models of Brucellosis and Brucella Induced Arthritis}

Mechanisms underlying arthritic responses induced by Brucella have been hindered by the lack of relevant mouse models, as wild-type mice infected with conventional doses $\left(10^{4}-10^{6}\right.$ colony forming units [CFUs]) by systemic routes do not develop joint inflammation $(58,70)$. Although mice are the best studied model of brucellosis, they are naturally resistant to Brucella infection and develop disease differently than humans or ruminants. One study suggests that upon systemic infection, $B$. melitensis is the most virulent Brucella spp. in mice followed by $B$. abortus with $B$. suis remaining the least virulent (71). In contrast to human brucellosis, mice tend to develop a transient acute infection that peaks around 23 weeks. Mice will naturally resolve infection, but it may persist for over 6 months. $(72,73)$. Additionally, much of the sequelae observed in chronic human brucellosis has not been reported in WT murine infection models (73). Rats, guinea pigs and monkeys have also been used as laboratory research models of Brucella infection, 
but to our knowledge do not develop arthritis (11). Furthermore, young cattle can develop arthritic complications upon administration of vaccine strain S19 directly into their joints (74). However, it is difficult to measure immune responses in cattle due to the limited availability of genetically similar animals, and logistical and financial complications inherent to working with cattle under BSL-3 conditions. Others have observed that WT BALB/c mice infected intraperitoneally (i.p.) with $1 \times 10^{7}$ CFUs of $B$. melitensis can develop articular brucellosis at 26 weeks postinfection. Studying immune responses in this model is challenging as arthritis development is sporadic, and it is difficult to experimentally modulate immune responses past 4 weeks post-infection (75). Additionally, detectable Brucellae were found in the bones and limbs of this model but obvious Brucella joint burdens were not evident, thus making it difficult to determine the effects of immune responses on Brucella clearance (75). Moreover, i.p. Brucella infection of IFN- $\gamma$ deficient mice results in arthritis of ankle joints as early as 15 days post-infection. It is suspected that IFN- $\gamma$ deficiency allows for the hematogenous spread of Brucella, resulting in infection and subsequent inflammation of the joints (58).

\section{Inflammation and the Inflammatory Response to Brucella}

Inflammation is a natural process induced by the body's immune system in response to cellular damage or foreign antigen, and is a vital part of tissue homeostasis as it eliminates microbial pathogens, clears cellular debris and initiates tissue repair. During an inflammatory stimulus, such as Brucella infection, immune cells like neutrophils and monocytes are recruited to the site of the irritant 
and secrete cytotoxic molecules. This is toxic to bacterial pathogens but also leads to tissue damage and release of damage associated molecular patterns (DAMPS) which can initiate increased recruitment of inflammatory infiltrates. Thus, inflammation has to be tightly regulated in order to prevent this vicious cycle of tissue debilitating immune cell recruitment (76). In the case of arthritis, inflammation is reoccurring leading to pathology of the joint.

The common attributes of inflammation are described as redness, warmth, pain, recruitment/activation of leukocytes, and localized leakage of plasmaprotein-rich fluid into inflamed tissues (swelling) (77). Upon contact with an inflammatory stimulus, cytokines such as IL-1 and TNF- $\alpha$ are released by tissue resident cells surrounding the antigen. IL-1 and TNF- $\alpha$ signal through endothelial IL-1R and TNFR to induce rearrangement of actin and tubulin, allowing endothelial cells to open up gaps between tight junctions. This allows leakage of plasma proteins into the affected tissues. Furthermore, IL-1 and TNF- $\alpha$ production activate endothelial tissues to vasodilate and upregulate E-selectin and P-selectin (77). Upregulation of these molecules causes weak tethering of circulating leukocytes to the endothelium inducing a slow rolling motion and finally adhesion to endothelial integrins (78). Along with tissue activation, increased blood flow due to vasodilation culminates in leukocyte extravasation $(78,79)$. When the cell is stationary, it can migrate into the tissue when the proper signals are presented. This process not only aids in cell entry into the tissues but also helps activate inflammatory cells such as neutrophils (80).

As neutrophils adhere to the epithelial wall, migration into the tissue, or 
diapedesis, is initiated through contact with neutrophil CXCR2 receptor ligands such as CXCL1 and CXCL2 $(78,79)$. Although circulating neutrophils are continuously present in the blood, the majority of neutrophils and neutrophil precursors are stored in the bone marrow. Thus, during an infection robust neutrophil egress from the bone marrow needs to occur. In addition to CXCR2 ligand release at or near the epithelium, these chemokines are also released into the blood and signal to the bone marrow to promote neutrophil maturation and release. Following passage through the epithelium, inflammatory cells such as neutrophils migrate to sites of infection or tissue damage guided by a chemokine gradient (78). Inflammatory cells can also follow other chemotactic gradients like those established by lipid mediators such as eicosanoids $(81,82)$.

Once in the tissue, bacteria can be eliminated or restricted by neutrophil phagocytosis, and or by trapping them in extruded DNA termed neutrophil extracellular traps (NETs) (83). Neutrophils can also elicit bactericidal activity by releasing reactive oxygen species (ROS), matrix metalloproteinase (MMPs) and granules such as myeloperoxidase, elastase, defensins, NO and $\beta$-glucuronidase. These proteins are typically released during neutrophil degranulation; however, lytic cell death, such as netosis, pyroptosis or necrosis results in robust granule and inflammatory protein release along with DAMPS. While in the tissue, neutrophils can also secrete additional chemokines and cytokines to amplify inflammatory cell recruitment $(84,85)$. Thus, it is critical for neutrophils to undergo apoptosis in order to turn off the inflammatory response and prevent pathology.

Monocyte migration is similar to neutrophils as chemotactic signals, 
selectins and integrins promote their transmigration into infected, inflamed and/or damaged tissues. CCL2 is the most common chemokine involved in monocyte recruitment, consequently causing monocyte release from the bone marrow, diapedesis into affected tissues, and differentiation into macrophages (86). While in affected tissues macrophages, especially those primed with IFN- $\gamma$, phagocytose bacteria and make bactericidal molecules such as reactive nitrogen intermediates, ROS, and phagolysosomal enzymes (86-88). Macrophages are also strong producers of TNF- $\alpha$, IL- 6 and type I interferons and are thus inflammatory. Alternatively, macrophages also play an anti-inflammatory role as they are responsible for clearing dead and dying cells including apoptotic neutrophils (89). Macrophage efferocytosis of dead/dying cells not only reduces inflammatory DAMPs but leads to production of anti-inflammatory lipoxins, resolvins and protectins (90). Hence, inflammation is necessary for pathogen clearance but must be tightly regulated, as an endless cascade of inflammatory pathology can lead to disease such as rheumatoid arthritis (91).

While most pathogens are quickly identified and cleared by the innate immune system, Brucella evades extracellular immune recognition and lacks obvious pathogen associated molecular patterns (PAMPs). Therefore, an effective innate inflammatory response able to clear the pathogen is never mounted. Additionally, Brucella does not express proteins that directly harm host cells such as exotoxins, exoproteases or cytolysins; thus, pathology in most tissues is probably driven by a prolonged low-grade inflammatory response initiated by weak recognition of Brucella PAMPs (92). Brucella can however, induce robust 
inflammatory responses in some tissues such as the placenta and the joints (93). Despite systemic studies and some investigations that involve the placenta, examination of tissue specific inflammatory responses to Brucella are rare. Considering different tissues respond more robustly to Brucella antigen, it is vital to understand how Brucella spp. evade and are recognized by the immune system and whether inflammation induced by these stimuli are helpful for the clearance of Brucella in various tissues. Understanding the immune response to Brucella could lead to new therapies or treatments for Brucella-induced arthritis and inflammatory disease in general.

\section{Brucella's Evasive Intracellular Life Cycle}

Brucella is a stealthy pathogen equipped with many mechanisms to evade recognition and clearance by the host's immune system. One of Brucella's most effective strategies of evasion begins by establishing itself in an intracellular niche. Host macrophages and monocytes typically phagocytose Brucella via zipper-like phagocytosis and through lipid rafts $(94,95)$. Over $90 \%$ of the brucellae are quickly killed by these macrophages, but some bacteria are able to resist killing and work to establish an intracellular niche capable of replication (96). Brucella can also invade non-phagocytic cells, such as B-cells and trophoblasts, by utilization of Rho GTPases (97) While in the cell's phagosome, Brucella expresses a VirB type IV secretion system (T4SS) that secretes important effector proteins that modulate the endocytic pathway. Expression of the T4SS is induced upon acidification of the phagosome; thus, rather than phagosomal fusion with the lysosome and 
subsequent degradation of endosomal inhabitants, Brucella proteins reduce the lysosomal trafficking protein LAMP1 preventing phagolysosomal fusion $(98,99)$. After divergence from the phagolysosome fate, the phagosome containing Brucella traffics to the endoplasmic reticulum (ER), where it exchanges membrane components. This interaction makes the phagosomal membrane indistinguishable from the ER and other host cell organelles, allowing the development of a specialized compartment termed the Brucella containing vacuole capable of evading cytosolic detection. This vacuole then breaks free from the ER allowing safeguarded intracellular replication of the bacteria (99). Finally, Brucella sequesters glucose, lactate and other nutrients from the host cell in order to maintain its survival and replication $(100,101)$.

During chronic infection, Brucella preferentially replicates in alternatively activated macrophages (AAMs) which are cells typically involved in tissue repair. AAMs preferentially oxidize fatty acids as their energy source, allowing Brucella to exploit unutilized host cell glucose (100). Brucella may also replicate in classically activated macrophages (CAMs), or inflammatory macrophages which prefer glycolytic metabolism $(100,102,103)$. However, during acute infection it is unknown if Brucella prefers cells undergoing glycolysis or oxidative phosphorylation. Inflammatory macrophages, or macrophages activated by IFN- $\gamma$, are required for Brucella clearance, but considering the bacterium may exploit these glycolytic cells to establish a niche of replication, macrophages and Brucella have a paradoxical relationship (104). Hence, it is vital that intracellular host 
receptors recognize and mount an effective immune response in order to clear Brucella.

\section{Immune Recognition of Brucella}

Although Brucella evades the host immune system through intracellular invasion of phagocytes, pathogen recognition receptors (PRRs) are important initial responders to infection. PRRs recognize similar moieties on pathogens and therefore quickly signal and activate transcription factors to induce secretion of inflammatory cytokines, such as IL-1 and TNF- $\alpha$. Toll-like receptors (TLRs) are some of the best studied PRRs, but there is discrepancy in the current literature as to what TLRs control Brucella infection (105). This may be due in part to Brucella's ability to evade TLR activation by secreting proteins that block TLR signaling $(106,107)$. However, it is generally agreed that TLR2, TLR4, TLR6 and TLR9 can play an inflammatory role (108-112). TLR2 and TLR6 recognize Brucella lipoprotein (110), TLR4 can identify brucellar LPS (111) and TLR9 can recognize Brucella CpG DNA (109). These TLRs typically signal through the adaptor protein MyD88 in order to induce an inflammatory cytokine response (113). This robust inflammatory activity can be detrimental to the host as MyD88 signaling can result in arthritis and osteoclastogenesis in murine models of bacterium induced arthritis (114-116). However, it remains unknown if MyD88 or TLR signaling are involved in the induction of Brucella-induced arthritis.

Another important pathogen recognition system, especially for identifying intracellular pathogens, are inflammasomes. Inflammasomes use intracellular 
PRRs such as AIM2 (absent in melanoma 2) and NLRP3 (NACHT, LRR and PYD domains-containing protein 3) to detect cytosolic threats. Upon recognition of pathogen associated molecular patterns (PAMP), or some DAMPS, the ASC (apoptosis-associated speck-like protein containing a CARD) adaptor protein is recruited and forms a complex known as the inflammasome. Inflammasomes proteolytically cleave the central protein caspase- 1 into its active form. Caspase- 1 then activates pro-inflammatory cytokines IL-1 $\beta$ and IL-18 into their functional secreted structures, and can also induce a programmed form of lytic cell death called pyroptosis. Secretion of IL-1 $\beta$ and IL-18 are known to contribute to systemic Brucella restriction, however the role of pyroptosis during brucellosis remains unknown (117). Inflammasomes can recognize intracellular Brucella and other vacuolar pathogens by utilizing host guanylate binding proteins (GBPs) (118). GBPs perturb the membrane of the pathogen containing vacuole therefore exposing antigen to the cytosol which may then be recognized by inflammasomes (119). Caspase-11, otherwise known as the non-canonical inflammasome, directly recognizes bacterial LPS in the host cytosol (120). Like caspase-1, caspase-11 can induce pyroptosis and subsequent DAMPS which can indirectly activate the canonical inflammasome $(120,121)$. Similar to Brucella's weak stimulation of TLR4, Brucella LPS is not thought to strongly activate caspase-11 (122).

Considering MyD88 and caspase-11 can both act through Gram-negative LPS recognition, bacterial LPS is generally a strong activator of innate immune responses. However, due to Brucella's unconventional LPS structure, it is considered a virulence factor that allows Brucella to evade immune recognition 
$(123,124)$. Unlike enterobacteria which present a hexa-acylated lipid A and are therefore recognized by TLR4/MyD88 and caspase-11, Brucella expresses a hepta-acylated lipid $A$ and contains unusual very long chain fatty acids (VLCFAs). Additionally, Brucella LPS has a core oligosaccharide which acts as a shield to block LPS lipid A identification (125). Brucella LPS also contains an O polysaccharide which results in smooth colony formation and reduced host cell death, allowing survival of the infected host cell reservoir (126).

\section{Inflammatory Cytokines}

Brucella induced arthritis is characterized by heavy recruitment of inflammatory cells to the joint and the musculoskeletal structures surrounding the articular space (62). Similarly, neutrophils are the most abundant cells in the synovial fluid in murine models of rheumatoid arthritis $(127,128)$. Upon Brucella infection, macrophages and resident cells such as osteoblasts can produce vigorous chemoattractants such as IL-6 which recruits T-cells, and CCL2 which recruits monocytes. Additionally, these cells can also manufacture CXCR2 ligands, such as CXCL1 and CXCL2 which stimulate migration of PMNs (66, 129-131). It is not known, however, if these cytokine/chemokines lead to increased pathogenesis in the joints of brucellosis patients. Unlike rheumatoid arthritis where autoantibodies and autoreactive T-cells mediate pro-inflammatory cell recruitment (132), our group previously demonstrated joint Brucella infection in IFN- $\gamma$ deficient mice does not induce collagen auto reactivity. Rather, it is the host's immune response to Brucella that mediates focal inflammation (58). Although auto 
reactivity to collagen was not observed, it is unknown if adaptive immune cells such as B and T cells play a role in Brucella-induced arthritis or if they are needed for mediating resolution.

Inflammasome activated IL-1 $\beta$ and IL-18 can also induce cell mediated inflammation (120). IL-1 $\beta$ is known to recruit and activate inflammatory macrophages and other inflammatory cells (133), while IL-18 stimulates cytotoxicity in NK cells and can prime T-cell IFN- $\gamma$ production (134). IL-1 $\beta$ and IL18 are protective against systemic Brucellla burdens but they may also elicit overwhelming joint inflammation, $(117,135)$ as they contribute to the development of rheumatoid arthritis (136-138). IL-1 $\beta$ is elevated in the joints of brucellosis patients and can also induce inflammation in septic arthritis $(66,139)$. Additionally, IL-1 is partially responsible for joint inflammation in IFN- $\gamma$-deficient mice intraperitoneally infected with Brucella, but is also required for systemic Brucella clearance (58). It is currently unknown if IL-1 $\beta$ and IL-18 induce Brucella clearance in the joints or if they mediate arthritis in WT infected mice.

IFN- $\gamma$ is one of the best documented cytokines known to aid in the clearance of brucellosis $(58,140-142)$, as IFN- $\gamma$ promotes macrophages production of ROS and nitric oxide (NO) both which work to eradicate intracellular Brucella (143). Tcells are thought to be the primary producers of IFN- $\gamma$ during Brucella infection and are thus vital for the restriction of Brucella replication. Polymorphisms in IFN- $\gamma$ are associated with an increased risk of contracting brucellosis and developing Brucella-induced arthritis (144-147), and T-cell exhaustion, seen during chronic brucellosis correlates with diminished IFN- $\gamma$ production $(148,149)$. Similarly, 
IFN- $\gamma \mathrm{R}^{-/-}$and T-cell-deficient mice are individually unable to control systemic Brucella burdens (142). T-cell and/or NK cell induction of IFN- $\gamma$ is often induced by the synergistic signaling of IL-12 and IL-18 through their respective receptors (150, 151); however, it is not known if this is the protective mechanism of IL-18 seen during systemic infection. Additionally, not much is known about the role of IFN- $\gamma$ in the joint. Intraperitoneally infected IFN- $\gamma$-deficient mice develop arthritis but it is unclear if ablation of IFN- $\gamma$ allows Brucella to access the joint, as it is protective in systemic infection, or if there is a local role for IFN- $\gamma$ within joint tissues (58).

Apoptosis is typically the preferred mode of clearance of host cells infected with intracellular pathogens, as it can kill the infected host cell and the bacteria harbored inside while resulting in minimal damage to host tissues (152). Apoptosis can be a result of Brucella infected osteoblasts $(152,153)$; however, there are additional reports that Brucella infection enhances macrophage survival in order to maintain its intracellular niche (154). In vitro Brucella infection can also promote prolonged cell survival of synoviocytes, (155) a phenomenon also seen during rheumatoid arthritis (156). Although Brucella may inhibit host cell death to evade immune recognition, Brucella may need to induce host death in order to disseminate. Studies suggest some natural dissociation of Brucella LPS from smooth to rough occurs, promoting macrophage egress and allowing dissemination of smooth Brucella which did not dissociate $(126,157)$. Conflicting reports on Brucella's induction of host cell survival indicates Brucella may only induce cell death in a tissue or cell specific manner and the nature of cell death induced by Brucella remains largely unexplored. 


\section{Purpose and Experimental Approach}

Treatment of unresolved sterile arthritis such as rheumatoid arthritis or osteoarthritis involves eliminating or dampening host inflammatory responses. While inflammation may inflict severe articular pathology to the host during infectious arthritis, inflammation may also be vital for pathogen restriction. This creates a paradox when identifying appropriate treatments for brucellar arthritis as administration of anti-inflammatories may increase bacterial replication in the host. Thus, it is vital to characterize what inflammatory responses are helpful and harmful to the host. Thus, the focus of this research was to understand the inflammatory mediators inducing inflammation and arthritis, and to determine what inflammatory responses are needed in order to clear Brucella during brucellosis. We describe experimental Brucella-induced arthritis as an effective tool in understanding both adaptive and innate inflammatory responses to Brucella as swelling correlates with inflammation and can be monitored in longitudinal studies. 


\section{CHAPTER II}

\section{Materials and Methods}

\section{Bacterial strains and growth conditions}

All experiments with live Brucella were performed in biosafety level 3 (BSL3) facilities. B. melitensis $16 \mathrm{M}$ and B. abortus 2308 , obtained from Montana State University (Bozeman, Montana), and B. suis 1330, obtained from BEI Resources (Mannasas, VA), were grown for 3 days at $37^{\circ} \mathrm{C} / 5 \% \mathrm{CO}_{2}$ on Brucella agar (Ba) (Becton Dickinson). Colonies were picked from $\mathrm{Ba}$ plates, and strains were cultured in Brucella broth (Becton Dickinson) overnight at $37^{\circ} \mathrm{C}$ with shaking. The overnight Brucella concentration was estimated by measuring the OD at $600 \mathrm{~nm}$, and the inoculum was diluted to the appropriate concentration in sterile phosphatebuffered saline (sPBS). Actual viable titer was confirmed by serial dilution of the Brucella inoculum onto Ba plates.

\section{Deletion and complementation of the $B$. melitensis L-lactate dehydrogenase gene (IIdD)}

The IIdD gene in B. melitensis 16M (BMEII0377) was mutated using a nonpolar, unmarked gene excision strategy. Briefly, a 1250 bp gBlock fragment was synthesized by IDTDNA containing from 5'-3': 600 bp upstream of $/ I d D$, then the first and last three codons of $I I d D$, followed by $\sim 600$ bp downstream of $I / d D$ (Table 2). In addition, the 5 ' end of the gBlock fragment contained $30 \mathrm{bp}$ 
homologous to $30 \mathrm{bp}$ upstream of the BamHI site in pNTPS139 and the 3' end of the gBlock contained $30 \mathrm{bp}$ homologous to the $30 \mathrm{bp}$ downstream of the Sall site of pNTPS139. The suicide plasmid pNTPS139 (158) was digested with BamHI and Sall. The gBlock fragment and digested pNPTS139 were then ligated using the NEB Hi-Fi DNA Assembly Kit according to manufacturer's instructions (New England Biolabs). This plasmid was then introduced into B. melitensis $16 \mathrm{M}$, and merodiploid transformants were obtained by selection on Brucella agar plus 25 $\mu \mathrm{g} / \mathrm{ml}$ kanamycin. A single kanamycin-resistant clone was grown overnight in Brucella broth and then plated onto Brucella agar containing 6\% sucrose. Genomic DNA from sucrose-resistant, kanamycin-sensitive colonies was isolated and screened by PCR using primers (IIdD screen forward, IIdD screen reverse) in Table 3 for loss of the IIdD gene. Genetic complementation of the IIdD mutation was performed by expressing wild-type $/ I d D$ from its native promoter in pBBR1MCS-2 (159). The IIdD gene, along with the native promoter, was amplified from $B$. melitensis $16 \mathrm{M}$ genomic DNA by PCR using primers (IIdD clone forward and IIdD clone reverse) in Table 3. These primers also contained overlapping sequences to the Sacl and Xhol sites of pBBR1MCS-2. The resulting PCR fragment was then ligated with Sacl/Xhol digested pBBR1MCS-2 via the NEB Hi-Fi Assembly kit. This construct was sequenced and then introduced into $B$. melitensis $\Delta / / d D$ by conjugation. 


\begin{abstract}
GCGCCAGAAAGCTTCCTGCAGGATATCGTGCTTCATATTTGTCCGTTGCCGTGTTGTAGCGCATGGG ATGGGTAAGGCGCCCCGTATTTTCCAGATCGTAATCCGTCCATTCCCGAAGCTTGGAAACCGTGTGT TCGCGAAAAAAGGCAGGCGTAGTGCGCCGGTCCGTTGCCTCCCAGGCAACGGCCTTGACGCCGTT TTCACAAAATTCAAAAGACGAGCCATGTTCGGGATCACCCCATGCACAACCGGGACAATCGAACCCA TCCGGCTGATTGGCTTTCAAAAGCGCACGGGCGCCAGCCAGCGGTGCACCGCTGGCCAGCAATTG CTGGCCACAGCTTTTCAGCGCACCCCATCCGCCTGCCGCGCTGGAACGTTTGCCGATAAAACCGGT TTTTTTACCCTTGCTCATCTCTTCATCCTGTAAGCACAATGCCTGGCCGCCATGACCTGCCAGCAAAT TGTGCCGGGGCGGGAGCCAATAGCATAAGAAGAAAAGTGCACAGCTTCAATTAACCGCGAGCCATT TGTTATCGAATTGCTTGTTGGGACTTGATTTCAAGCGCGCGATCCGTCATTCGATTGGTAAAGAAAAC TGACCACAAAGGGTGGGATGATGTCCGATTTCTGATCTATCCACCAACAGATATAAGGCCCGATAAT AGTCGGGCCTTATTCCAGCTTTTTTAATAAATATCTTGGTTTATTATGCCTGCGACTGAGTTAACACCA GAAGCCGCGCAGCGATTAAGCTTTTTCAGGGCATAATACGCTAGCATCTTGGCAACTCTAACATAAT GAAATTTTAAGTTAACACTTATATAAAATAACCAATATTTAAGCATATCAGTTTACATCTCCGACCGAG CGGTATGAAAACCGTATATTTTTTTTGGGCTGGAGTGAAACACATGAAACGCGGTTGCGCCATTGCGG TCATGATATGCGGTTTAATCACAAGCGTGAGCGCGGCATCAGCCGCTGACCTCATAGTTCAGGAACC CGTTTTTGAGCCTTTACCCCAGCCAGCCCTTGCGGGCTGGTATCTCCGGGGCGATATCGGCTACAA TTTTAAATCGAAAACCGGCGGCAAGTGGGATTTCTGGAACCAGTTTGAAGAACCCTATCGCGGGGTT GACGACACCTTCAACTATGATGATTTCTCGCTGAAAGGCGGAGCCTCCTATGGGGTCGGCGTCGGC TATCGCTTCAACGACATCCCACTAGTGAGTCGTATTACGTAGCTTGG
\end{abstract}

Table 2. gBlock construct for deletion of IIdD.

Underlined regions denote homology to pNPTS139. Underlined/bold region denotes first 3 codons of $I / d D$. Bold/italics region denotes last 3 codons of $I / d D$. 


\begin{tabular}{|l|l|l|}
\hline Primer name & 5'-3' sequence & Purpose \\
\hline GAPDH-F & $\begin{array}{l}\text { GTG GAC CTC ATG GCC TAC } \\
\text { AT }\end{array}$ & qRT-PCR \\
\hline GAPDH-R & $\begin{array}{l}\text { GGG TGC AGC GAA CTT TAT } \\
\text { TG }\end{array}$ & qRT-PCR \\
\hline iNOS-F & $\begin{array}{l}\text { TCC TGG ACA TTA CGA CCC } \\
\text { CT }\end{array}$ & qRT-PCR \\
\hline iNOS-R & $\begin{array}{l}\text { CTC TGA GGG CTG ACA CAA } \\
\text { GG }\end{array}$ & qRT-PCR \\
\hline IIdD screen-F & $\begin{array}{l}\text { ATT TGT CCG TTG CCG TGT } \\
\text { TG }\end{array}$ & $\begin{array}{l}\text { Confirming deletion of } \\
\text { IldD }\end{array}$ \\
\hline IIdD clone-F* & $\begin{array}{l}\text { TCC GCC TTT CAG CGA GAA } \\
\text { AT }\end{array}$ & $\begin{array}{l}\text { Confirming deletion of } \\
\text { IldD }\end{array}$ \\
\hline $\begin{array}{ll}\text { ATT GTG CCG GGG CGG GAG } \\
\text { CC }\end{array}$ & Complementation of IIdD \\
\hline IIdD clone-R* & $\begin{array}{l}\text { GTA CCG GGC CCC CCC GAT } \\
\text { CAG AAA TCG ATC GAA TGG }\end{array}$ & Complementation of IIdD \\
\hline
\end{tabular}

Table 3. Primers used in this study.

*Underlined regions denote homology to pBBR1MCS-2 


\section{Mouse strains}

Experiments were conducted using 6- to 12-week-old age- and sexmatched mice on a C57BL/6 or BALB/c background. Breeding pairs of MyD88 ${ }^{-/}$, caspase- $1 / 11^{-/-}$, caspase- $11^{-/-}, \mathrm{IL}-1 \mathrm{R}^{-/-}, \mathrm{IL}-18^{-/-}, \mathrm{NLRP3}^{-/-}$and $\mathrm{AIM} 2^{-/-}$mice (on C57BL/6 backgrounds) and $\mathrm{CXCR} 2^{-/-}$mice (on a BALB/c background) were obtained from the Jackson Laboratory (Bar Harbor, ME). Breeding pairs of IFN-Yَ 1- and heterozygous $\mathrm{CXCR}^{+/-}$mice (on C57BL/6 backgrounds) were obtained from Jackson Laboratory. Heterozygous $\mathrm{CXCR}^{+/-}$mice were intercrossed with IFN- $\mathrm{Y}^{-/-}$ mice to obtain IFN- $\gamma^{-/-} / \mathrm{CXCR} 2^{-/-}$mice. Breeding pairs of IL-1R/IL-18 $8^{-/-}$mice were gifted by Dr. Edward Miao (University of North Carolina, Chapel Hill, NC). Rag $1^{-/ /}$, $\mu \mathrm{MT}^{-/-}, \mathrm{CCR}^{-/-}, \mathrm{Perf}^{--}$and Nos2 ${ }^{-/-}$mice on a C57BL/6 background were also purchased from Jackson Laboratory.

\section{Mouse infections}

In some experiments mice were infected i.p. with $200 \mu \mathrm{l}$ of $\sim 1 \times 10^{5} \mathrm{CFUs}$ of B. melitensis $16 \mathrm{M}$ in sPBS. In other studies mice were infected in both rear footpads with a volume of $50 \mu$ containing $1 \times 10^{3}, 1 \times 10^{4}, 1 \times 10^{5}$ or $1 \times 10^{6} \mathrm{CFU}$ of $B$. melitensis, $B$. abortus or B. suis (160). After infection, all mice were maintained in individually ventilated cages under high-efficiency particulate air-filtered barrier conditions of $12 \mathrm{~h}$ of light and $12 \mathrm{~h}$ of darkness in animal BSL3 facilities and were provided with sterile food and water. All studies were conducted in accordance with University of Missouri Animal Care and Use Committee guidelines. 


\section{Antibody neutralizations/ treatments}

In order to neutralize IFN- $\gamma$, in i.p. infected mice, mice were treated with antiIFN-y antibody (clone XMG1.2, BioXCell) via intraperitoneal administration of 0.25 mg 1 day before infection and 3 times per week thereafter. Mice neutralized of IFN$\gamma$ during footpad infections were treated with anti-IFN- $\gamma$ antibody (clone XMG1.2, BioXCell) via intraperitoneal administration of $0.5 \mathrm{mg} 1$ day prior and 3 days after Brucella infection. To neutralize IL-18 mice were administered $2.5 \mathrm{mg}$ of anti-IL18 antibody (clone YIGIF74-IG7, BioXCell) into the peritoneum one day prior to infection. NK cells and $ү \delta$ T-cells were neutralized via i.p. administration of $0.5 \mathrm{mg}$ of anti-NK-cell antibody (clone PK136, BioXCell) or anti-үठ T-cell antibody (clone UC7-13D5, BioXCell) respectively, 1 day prior and 3 days after Brucella infection. Control mice for antibody depletion experiments received Rat IgG (Southern Biotech) as an isotype control. For eicosanoid experiments mice received intraperitoneal delivery of $2 \mathrm{mg} / \mathrm{kg}$ indomethacin (Sigma) on day -1 and daily thereafter. To inhibit glycolysis in vivo mice were administered $100 \mathrm{mg} / \mathrm{kg}$ of 2deoxyglucose i.p. (2-dG) on days 1-6 post-infection. For in vivo nitric oxide synthase inhibition, mice were given drinking water containing $2.5 \%$ aminoguanidine hemisulfate salt (AGHS, Sigma) (161) 14 days prior to infection and throughout the experimental time course.

\section{Bacterial burdens}

At various times after infection, mice were euthanized, and spleens and mouse paws (following skin removal) were harvested. Spleens and paws were 
homogenized mechanically in SPBS (160). A series of 10-fold serial dilutions were performed in triplicate in SPBS and plated onto Ba. Plates were incubated 3-4 days at $37^{\circ} \mathrm{C}$, colonies were enumerated, and the number of CFUs/tissue was calculated.

\section{Macrophage generation and infections}

Bone marrow derived macrophages (BMDMs) were derived by flushing the bone marrow from femurs and tibias of C57BL/6 mice with sPBS. Isolated bone marrow was then cultured in complete media (CM; RPMI 1640, 10\% fetal bovine serum (FBS), $10 \mathrm{mM}$ HEPES buffer, $10 \mathrm{mM}$ non-essential amino acids, $10 \mathrm{mM}$ sodium pyruvate) containing $30 \mathrm{ng} / \mathrm{ml}$ recombinant murine M-CSF (Shenandoah). After 3-4 days of culture fresh CM containing $30 \mathrm{ng} / \mathrm{ml} \mathrm{M-CSF}$ was added to culture flasks. Adherent cells were then collected at 6-7 days post-bone marrow harvest by adding $0.05 \%$ Trypsin (Life Technologies). Cells were plated at $1 \times 10^{6} \mathrm{cell} / \mathrm{ml}$ in fresh $\mathrm{CM}$ and left to adhere overnight. Cells were infected with a multiplicity of infection (MOI) of 100 B. melitensis 16M. Cells were infected for 6 hours, washed with sPBS, CM containing $50 \mu \mathrm{g} / \mathrm{ml}$ gentamicin was added, and cells were incubated for 30 mins. Cells were then washed with sPBS and left to incubate in CM containing $2.5 \mu \mathrm{g} / \mathrm{ml}$ of gentamicin for the remainder of the experiment. In some experiments 2-dG or SNAP was added as indicated. 10\% FBS was used for all studies unless cell death was assayed or western blots were performed (Fig 23A-D, Fig 30G, and Fig 24C), in which case $2 \%$ FBS was used. Supernatants were harvested and intracellular Brucella in lysates were enumerated at 24 or 48 
hours post-infection. Supernatants were used for L-Lactate quantification as described below. Measurements of IL-1 $\beta$ were obtained using a Mouse IL-1 $\beta$ ELISA Ready Set Go kit according to the manufacturer's instructions (Invitrogen) and LDH release was measured utilizing the Cytotoxicity Detection Kit plus (LDH) adhering to the vendor's protocols (Roche). Additionally, cell lysates and supernatants were used for western blots. To determine bacterial burdens BMDMs were washed with sPBS, lysed in $\mathrm{ddH}_{2} \mathrm{O}$ and $\mathrm{CFU}$ s enumerated by serial dilutions onto $\mathrm{Ba}$. For competitive index experiments, both $\Delta / / d D$ and the $I / d D$ complemented strain (containing kanamycin resistance $(\mathrm{KanR})$ ) were combined in a 1:1 ratio and titered at the time of infection by plating inoculum on regular $\mathrm{Ba}$ and Ba containing $25 \mu \mathrm{g} / \mathrm{ml}$ kanamycin. Phagocytosis was determined by plating cell lysate collected at $0 \mathrm{hr}$ post-infection on regular $\mathrm{Ba}$ and $\mathrm{Ba}$ with kanamycin. Cell lysates were also collected at 48 hours post-infection, and plated onto regular $\mathrm{Ba}$ and Ba with kanamycin then CFUs were enumerated. The final competitive index was calculated by comparing the relative ratio of the two strains at $48 \mathrm{hrs}$ postinfection relative to their phagocytosis.

\section{Lactic acid quantification}

In order to remove cell debris BMDM supernatants were centrifuged at $13,000 \mathrm{Xg}$ for $10 \mathrm{~min}$. Supernatants were then deproteinated using a Vivaspin 500 10K MWCO filter (Fisher) according to the manufacturer's instructions. L-Lactate was measured using the L-Lactate kit (Eton Bio) according to the manufacturer's instructions. 


\section{LPS purification and transfection}

LPS was purified from the $B$. abortus S19 vaccine strain (obtained from the University of Wyoming) using hot-phenol extraction (162) Briefly, S19 was suspended in deionized water at $66^{\circ} \mathrm{C}$ and then combined with $90 \%$ phenol (Sigma), preheated to $66^{\circ} \mathrm{C}$. The suspension was stirred for 20 minutes, followed by cooling in an ice bath before centrifugation. The phenol layer was aspirated using a 16 gauge cannula and cellular debris removed via filtration using a Whatman \#1 filter. The LPS was then precipitated via addition of methanol (Sigma) containing $1 \%$ methanol saturated with sodium acetate (Sigma) and allowed to incubate for $2 \mathrm{hr}$ at $4^{\circ} \mathrm{C}$ before centrifugation. The precipitate was stirred with deionized water for 18 hours at $4^{\circ} \mathrm{C}$. Following incubation, the LPS was centrifuged, the supernatant reserved and maintained at $4^{\circ} \mathrm{C}$, while the precipitate was resuspended in deionized water and stirred for 2 hours at $4^{\circ} \mathrm{C}$. The supernatant solution was recovered as above, and pooled with the reserved supernatant sample. Trichloroacetic acid (sigma) was added to the supernatant containing the crude LPS, stirred for 10 minutes, and the precipitate removed via centrifugation. The supernatant solutions were dialyzed against deionized water and then freeze dried.

Peritoneal macrophages were isolated by injecting mice with $1 \mathrm{ml}$ of aged thioglycolate (163). 4 days post-injection peritoneal macrophages were isolated by washing the peritoneal cavity with $10 \mathrm{mls}$ of sPBS. Macrophages were resuspended in $\mathrm{CM}$ and cultured at $1 \times 10^{6} \mathrm{cells} / \mathrm{ml}$ and primed overnight with 1 $\mu \mathrm{g} / \mathrm{ml}$ Poly I:C (Invivogen). Cells were washed and then cultured in Opti-MEM 
reduced serum media (Thermo-Fisher). Purified Brucella LPS was packaged in Fugene 6 (Promega) at room temperature for 15 minutes according to manufacturer's instructions. Wells receiving $10 \mu \mathrm{g} / \mathrm{ml}$ LPS received a final concentration of $0.25 \%(\mathrm{v} / \mathrm{v})$ Fugene 6 , while wells receiving $100 \mu \mathrm{g} / \mathrm{ml}$ LPS received a final concentration of $2.5 \%$ Fugene. Cells were incubated for 9 hours at which time LDH release was measured as described above.

\section{Immunoblots}

Supernatants and BMDMs were evaluated at 24 hours post-infection. BMDMs were lysed in RIPA buffer (Thermo), harvested, and total protein normalized using BCA Protein Assay Kit according to the manufacturer's instructions (Thermo). Caspase-1 westerns were run on 15\% acrylamide gels. Blots were probed with anti-Caspase-1 (p20) (casper-1) (Adipogen) diluted 1:500 and then with Peroxidase-conjugate Goat Anti-Mouse IgG (Jackson Immuno Research) diluted 1:5000. Detection was performed with SuperSignal West Femto Maximum Sensitivity Substrate (Thermo).

\section{Extraction of RNA and RT-PCR analysis of joint homogenates}

Joints were homogenized in TRI reagent (Sigma). $100 \mu \mathrm{l}$ of chloroform (Sigma) was added to $500 \mu \mathrm{l}$ of TRI reagent, incubated for $10 \mathrm{mins}$, and centrifuged at $15000 \mathrm{RPM}$ for 15 mins at $4^{\circ} \mathrm{C}$ (163). The top RNA containing layer was harvested and mixed with $700 \mu \mathrm{l}$ of isopropanol (Acros organics). RNA was further purified with an RNeasy Mini Kit (Qiagen). cDNA was generated using the 
superscript III First Strand Synthesis System (Invitrogen) using oligodT primers. Primers for iNOS and GAPDH were designed using NCBI Primer Blast. Primer sequences are included in Table 3. Relative specific mRNA was quantified by measuring SYBR green incorporation during real-time quantitative reverse transcription-PCR (qRT-PCR) (performed in duplicate for each sample) using the comparative threshold method (164).

\section{Assessment of pathology}

Ankle swelling was evaluated in relation to basal joint measurements made prior to infection as have described (160). Ankle swelling was measured at various time points by collective measurements of both tibiotarsal joints. The difference of the recorded measurement from basal was reported as mean joint swelling. Histology was performed on both mouse ankles, in which skin was removed prior, and on mouse tails. Tissues were fixed in $10 \%$ buffered zinc formalin, decalcified in $15 \%$ formic acid, rinsed in tap water, dehydrated with an alcohol gradient and clearing agent then embedded in paraffin. Tissue sections $(5 \mu \mathrm{m})$ were mounted on glass slides, and serial sections were stained with hematoxylin and eosin (H\&E) and covered with a coverslip with aqueous mounting medium. Tissues were evaluated for inflammation (neutrophils, macrophages, lymphocytes) which involved multiple tissues (synovial membrane, bone, tendons, skeletal muscle). Two sections at different levels of each ankle joint and associated structures were evaluated for each mouse by histopathology and scored for lesion severity (inflammation). The following scale was used: $0=$ none (no inflammation); $1=$ 
minimal with inflammation involving $<5 \%$ of tissue; $2=$ moderate with focally extensive areas of inflammation (5\% to $25 \%$ of tissue and involving 1 or more tissues); 3 = moderate to severe with focally extensive areas of inflammation ( $>25 \%$ to $50 \%$ of tissue and involving multiple tissues and mild distention of tissues associated with joint); 4 = severe with large confluent areas of inflammation ( $>50 \%$ of tissue and involving all tissues and severe distention of tissues associated with joint) (160). For enzyme immunohistochemical (IHC) staining, mouse tail tissue sections $(5 \mu \mathrm{m})$ were mounted on Plus Charged glass slides and sections were deparaffinized using appropriate buffer and rinsing between each immunostaining step. Samples were rehydrated in xylene, absolute alcohol, 95\% alcohol, and rinsed with distilled water. Endogenous peroxidase was blocked with $3 \% \mathrm{H}_{2} \mathrm{O}_{2}, 5 \%$ BSA and slides were incubated with $4.7 \mu \mathrm{g} / \mathrm{ml}$ of rat anti-Ly-6G antibody (clone 1A8, BioXCell) for 60 minutes. Slides were then incubated with secondary biotinylated donkey-anti-rat antibody, followed with incubation in streptavidin-HRP (Dako). Slides were developed with diaminobenzidine (DAB+, Dako) and lightly counterstained with hematoxylin 1 and coverslipped with aqueous mounting media. Three sections of tail at different tissue levels (bone and associated structures) were evaluated and scored for each mouse by a histopathologist. For neutrophil infiltration (IHC), the following scoring system was used (labeling of neutrophils in normal bone marrow was excluded from evaluation): $0=$ none (no labeled cells); 1 = minimal numbers of labeled cells; 2 = moderate numbers of labeled cells; 3 = large numbers of labeled cells and skeletal muscle along with periarticular inflammation. 


\section{Flow cytometry}

Rear legs were de-gloved, dislocated at the hip, muscle trimmed, and put into a sterile 4-mL PBS solution containing $125 \mathrm{U} / \mathrm{mL}$ collagenase (Sigma), 2 $\mu \mathrm{L} / \mathrm{mL}$ of DNase (I U/mL; Thermo Scientific), and $4 \%$ fetal bovine serum (FBS; Sigma) and incubated for 1 hour at $37^{\circ} \mathrm{C}$ with shaking. Joint slurry was put into sterile dishes, stripped of surrounding tissue, strained through an $80-\mu \mathrm{M}$ mesh, and washed with complete medium. Cells were re-suspended in RPMI 1640 medium with $0.1 \mathrm{mM} \mathrm{HEPES}$, and red blood cells were lysed with lysis buffer (final concentration, $0.15 \mathrm{M} \mathrm{NH} 4 \mathrm{Cl}, \quad 10 \mathrm{mM} \mathrm{KHCO} 3$, and $0.1 \mathrm{mM}$ ethylenediaminetetraacetic acid) (70). Cells were then washed and stained in fluorescence-activated cell-sorting (FACS) buffer (PBS and 2\% $\mathrm{FBS}$ ). Immunofluorescence staining was performed for 30 minutes at $4^{\circ} \mathrm{C}$, using a combination of the following fluorochrome-labeled monoclonal antibodies from eBioscience: F4/80 (BM8), Ly-6G (1A8), CD11b (M1/70) and CD45.2 (104), CD4 (GK1.5), CD8a (2.43), and B220 (RA3-6B2). Stained cells were then washed with FACS buffer and fixed overnight in $4 \%$ paraformaldehyde at $4^{\circ} \mathrm{C}$. After fixation TUNEL staining was conducted on some samples using the Click-iT plus TUNEL assay kit (Life technologies) according to the manufacturer's instructions then reconstituted in FACS buffer. Fluorescence was acquired on a CyAn ADP Analyzer (Beckman Coulter), and FlowJo (Tree Star) software was used for analysis. The following gates were used to detect the following cell types: Macrophages CD $45.2^{+} / \mathrm{CD} 11 \mathrm{~b}^{+} / \mathrm{F} 4 / 80^{+}$, neutrophils CD $45.2^{+} / \mathrm{CD} 11 \mathrm{~b}^{+} / \mathrm{F} 4 / 80^{+}, \mathrm{CD} 4$ 
T-cells CD45.2 $2^{+} / \mathrm{CD}^{-} / \mathrm{CD}^{+}, \quad$ CD8 $\quad$ T-cells $\mathrm{CD}^{+} 5.2^{+} / \mathrm{CD}^{-} / \mathrm{CD} 8^{+}, \quad$ B-cells CD $45.2^{+} / \mathrm{B}^{2} 20^{+}$.

Spleens were processed for intracellular flow cytometry by homogenizing splenic tissue in $900 \mu \mathrm{l}$ of sterile PBS. Tissue homogenates were strained through an $80 \mu \mathrm{m}$ mesh and washed with complete medium. Cells were resuspended in RPMI medium with $0.1 \mathrm{mM}$ HEPES, and red blood cells were lysed. Spleen cells were then washed and resuspended in $\mathrm{CM}$ and cultured for 4 hours at $37^{\circ} \mathrm{C} / 5 \% \mathrm{CO}_{2}$ with a cell stimulation cocktail containing phorbol 12 -myristate 13 acetate (PMA), ionomycin, brefeldin A, and monensin (Ebioscience). Cells were then stained for surface markers (anti-CD4, clone GK1.5 anti-CD8, clone 2.43) and fixed in paraformaldehyde. Cells were permeabilized with $0.2 \%$ saponin prior to intracellular staining for IFN-y (clone XMG1.2). Fluorescence was acquired on a CyAn ADP Analyzer (Beckman Coulter), and FlowJo (Tree Star) software was used for analysis

\section{Ankle processing for cytokine measurements}

Following skin removal, ankles from each mouse were excised, combined, and then homogenized in toto in 3 to $4 \mathrm{ml}$ of sPBS treated with Halt protease inhibitor cocktail (Thermo Scientific) (160). Homogenized tissues were then centrifuged at $2,000 \mathrm{~g}$ for $5 \mathrm{~min}$, supernatants were filter sterilized $(0.2 \mu \mathrm{m}$ pore $)$ and stored at $-70^{\circ} \mathrm{C}$ until analysis using a Luminex MagPix instrument with Milliplex magnetic reagents according to the manufacturer's instructions (Millipore). Luminex data were analyzed with Milliplex Analyst software (Millipore) and 
normalized to total protein levels determined by a bicinchoninic acid (BCA) protein assay (Thermo Scientific).

\section{Statistical analysis}

Focal inflammation incidence was analyzed by log-rank analysis on incidence curves. Statistical analysis of the difference between 2 mean values was conducted using a two-tailed Student $t$ test with significance set at $P \leq .05$, while comparisons of $\geq 3$ mean values was done using ANOVA, followed by Tukey's test (when all means were compared to each other) or Dunnett's test (when samples means were only compared with a control group) as described in the Figure Legends with significance set at $P \leq .05$. The statistical significance of differences in histology scores was determined by a Mann-Whitney $U$ test for 2 groups, or an ANOVA on ranks for $\geq 3$ groups. 


\section{CHAPTER III}

\section{CXCR2 Mediates Brucella-Induced Arthritis in IFN-Y- Deficient Mice}

\section{ABSTRACT}

Brucella spp. are facultative intracellular gram negative bacteria that cause brucellosis, a common global zoonosis. Infection of the joints is the most common focal complication of brucellosis in humans. The purpose of this study was to identify mediators of focal inflammation during brucellosis. Wild-type (WT) mice are naturally resistant to Brucella infection; therefore, we infected anti-IFN- $\gamma$ treated, or IFN- $\gamma^{-/-}$mice with Brucella to induce osteoarticular and musculoskeletal inflammation as has been previously described. Mice were infected intraperitoneally with $B$. melitensis and the clinical course of disease, histopathologic changes, and cytokine levels were compared among groups. Rag $1^{-/-}$mice (B and T cell deficient) and $\mu \mathrm{MT}^{-/-}$mice (B cell deficient) developed paw inflammation at a similar rate and severity as wild-type mice following infection with B. melitensis and treatment with anti-IFN-ץ. Joints from B. melitensis- infected IFN- $\gamma^{-/-}$mice had markedly increased levels of CCR2 and CXCR2 ligands. While anti-IFN- - -treated $\mathrm{CCR}^{-/-}$and WT mice behaved similarly, anti-IFN- $\mathrm{Y}$-treated $\mathrm{CXCR} 2^{-/-}$or IFN $-y^{-1-} / \mathrm{CXCR} 2^{-/-}$mice had strikingly reduced focal swelling relative to anti-IFN- - -treated WT or IFN- ${ }^{-/-}$mice, respectively. Additionally, neutrophil recruitment was dependent on CXCR2. Collectively these data demonstrate adaptive immune cells and CCR2 are dispensable, while CXCR2 is necessary for Brucella-induced focal neutrophil recruitment and inflammation. 


\section{INTRODUCTION}

Investigation of articular brucellosis has been hindered by the lack of relevant models, as wild-type mice infected with conventional doses of Brucella do not develop disease in the same way as humans or ruminants $(165,166)$. Previous work, has shown that genetic or antibody-mediated ablation of IFN- $\gamma$ in mice results in joint colonization and development of arthritis within two to four weeks after intranasal or i.p. infection with $\sim 2 \times 10^{4}$ CFUs B. melitensis or B. abortus (165). While humans have an intact IFN-Y gene, a single nucleotide polymorphism $(+874 \mathrm{~A} / \mathrm{A})$ that potentially subverts optimal NF-KB binding to the IFN- $\gamma$ gene and correlates with diminished IFN- $\gamma$ expression was found to be a brucellosis risk factor $(141,145-147)$. In addition, another IFN- $\mathrm{Y}$ polymorphism (5644A) associated with low IFN-ץ production was found to be overrepresented in patients with focal complications of brucellosis (144). Human patients with chronic brucellosis also have an impaired IFN-y response to Brucella antigen relative to patients at the onset of disease (148). Thus, suboptimal IFN-y responses may predispose both humans and mice to focal brucellosis.

It has been reported that neutrophils are the most abundant cells in the synovial fluid of mice with experimental rheumatoid arthritis $(127,128)$. Examination of synovial fluid or tissue from brucellosis patients reveals a mixture of polymorphonuclear (PMN) and mononuclear leukocyte infiltrates $(29,57,67$, 167). Neutrophil activation and recruitment to the joints is stimulated by proinflammatory cytokine/chemokine secretion into the synovium, consequentially 
leading to neutrophil release of granule contents, reactive oxygen species and other pro-inflammatory products that may drive inflammation (127).

Little information exists on the composition of cytokines/chemokines in the synovial fluid of human brucellosis patients. Previously, our group found that IL$1 \mathrm{R}^{-/-}$mice have reduced musculoskeletal inflammation when infected with Brucella and depleted of IFN-ץ (165). In addition, elevated levels of IL-1 $\beta$, CCL2 and CXCR2 ligands have been found in the synovial bursa of a human brucellosis patient with osteoarticular complications(66). In order to characterize the induction of Brucella-induced focal inflammation, we investigated the role of adaptive immunity and chemokine production. Here, we show that adaptive immune cells are dispensable, but CXCR2 is critical for Brucella induced-arthritis.

\section{RESULTS}

\section{Inflamed $\underline{B}$. melitensis-infected joints have increased levels of neutrophils}

To characterize infiltrating cells, flow cytometry was performed on joints from naïve and $B$. melitensis-infected mice. A marked increase in hematopoietic cells $\left(\mathrm{CD} 45.2^{+}\right)$, and in particular neutrophils, $\left(\mathrm{CD} 45.2^{+} / \mathrm{L} y-6 \mathrm{G}^{\mathrm{hi}}\right)$ was observed in Brucella-infected IFN- $\gamma^{-/-}$mice (Fig 1A-B) relative to infected wild-type mice. To a lesser extent there was a slight, but statistically significant, increase in $\mathrm{CD} 4^{+} \mathrm{T}$ cells in Brucella-infected WT and IFN- $\gamma^{-/-}$mice relative to naïve controls (Fig 1B). A significant increase in the proportion of $\mathrm{CD} 45.2^{+} / \mathrm{CD} 11 \mathrm{~b}^{+} / \mathrm{Ly} 6-\mathrm{G}^{-} / \mathrm{F} 4 / 80^{-}$cells was also seen in infected IFN- $\gamma^{-/}$mice, relative to infected wild-type animals, which may indicate an influx of monocytes (data not shown). 
A.

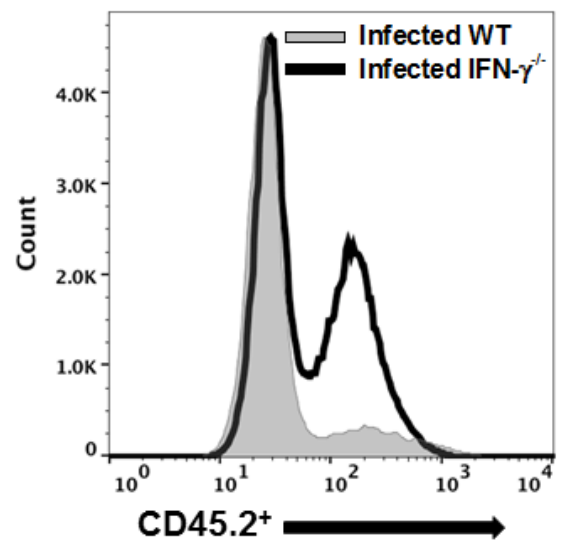

C.

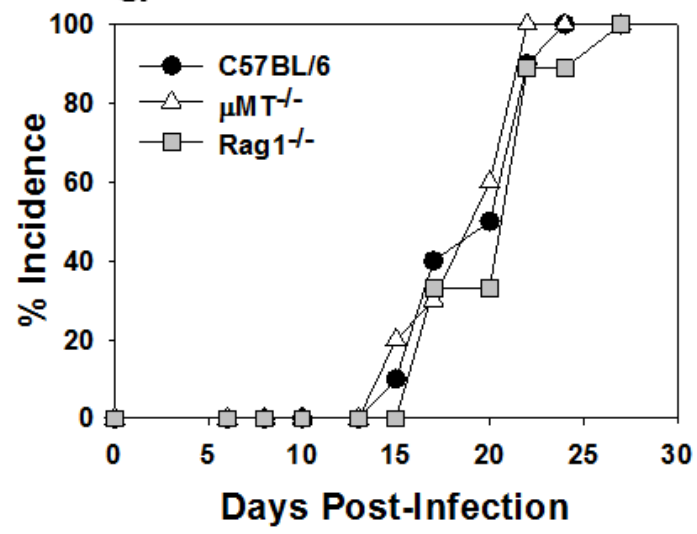

B.

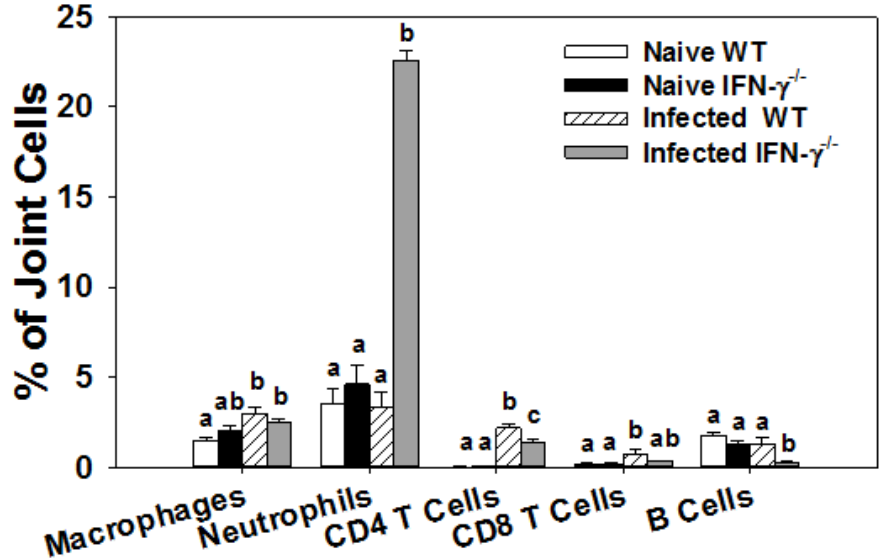

D.

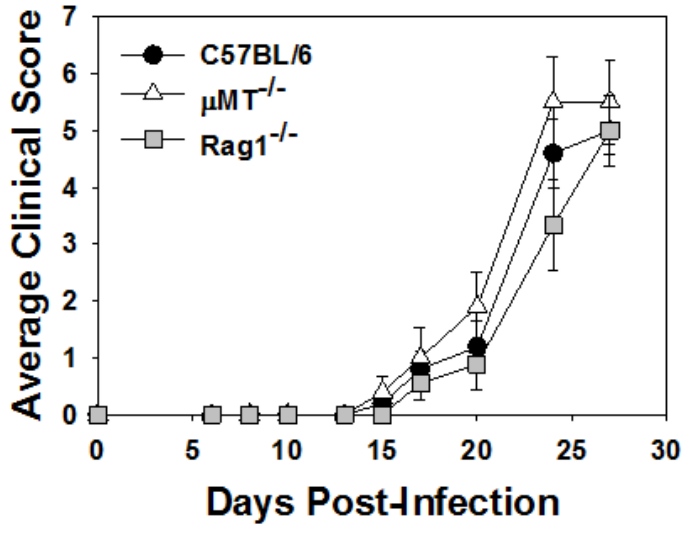

Figure 1. T and B cells are not required for Brucella-induced focal inflammation.

Flow cytometry was performed on joints from naïve C57BL/6 and IFN- $\gamma^{-1-}$ mice or from C57BL/6 and IFN- $\gamma^{-/-}$mice 28 days after i.p. infection with $\sim 1 \times 10^{5} \mathrm{CFUs}$ of Brucella melitensis $16 \mathrm{M}$ (A-B) ( $n=4-5$ mice/group). Within a cell type, means with the same letter are not significantly different from each other $P<0.05$. Error bars depict SEM. (C-D) Wild-type, $\mu \mathrm{MT}^{-/-}$and Rag $1^{-/-}$mice (all on a C57BL/6 background) were infected intraperitoneally (i.p.) with $\sim 1 \times 10^{5}$ CFUs of Brucella melitensis $16 \mathrm{M}$ and treated with anti-IFN- $\gamma$ ( $n=5-10$ mice/group). Incidence of paw swelling (C) and average clinical scores (D) were recorded over time. Error bars depict SEM. 


\section{$T$ and $B$ cells are not required for Brucella-induced focal inflammation}

To investigate a role for adaptive immune cells, wild-type, $\mu \mathrm{MT}^{-{ }^{--}}$(B cell deficient), and Rag $1^{-/-}$mice (B and T cell deficient) were infected i.p. with $\sim 1 \times 10^{5}$ CFUs of $B$. melitensis and treated with anti-IFN- $\gamma$ antibody as described in Methods. To assess inflammation, mice were monitored for swelling and redness at the tibiotarsal junction. Anti-IFN- $\gamma$-treated wild-type, $\mu \mathrm{MT}^{-{ }^{-/}}$, and Rag ${ }^{-{ }^{--}}$mice displayed similar incidence, degree of paw swelling and clinical scores (Fig 1C\&D). Brucella joint tissue loads and neutrophil infiltration, as measured by flow cytometry, were also similar between WT and $\mu \mathrm{MT}^{-/-}$mice at day 28 post-infection (data not shown). Tissue bacterial burdens and joint neutrophil infiltration was not measured in Rag $1^{-/-}$mice as $80 \%$ of animals succumbed to infection by day 28 (data not shown).

CCR2 and CXCR2 ligands are up-regulated in inflamed, B. melitensisinfected joints

To determine cytokines/chemokines that were induced during infection, paw homogenates from B. melitensis infected wild-type and IFN- $\gamma^{-/-}$mice were assessed for T-cell-associated cytokines, inflammatory cytokines and chemokines by Luminex at day 23 and 30 post-infection (Fig 2A-C). In infected wild-type mice, IL-13 and IL-2 were the only cytokines enhanced relative to infected IFN- ${ }^{-1-}$ animals (Fig 2A). Marked increases in the inflammatory cytokines, IL-1ß, IL-6 and TNF- $\alpha$ were seen in infected IFN- $\gamma^{-/-}$mice at days 23 and 30 post-infection (Fig 2B). Additionally, the chemokines CXCL2, CCL2, and CCL3 were also significantly 
A.

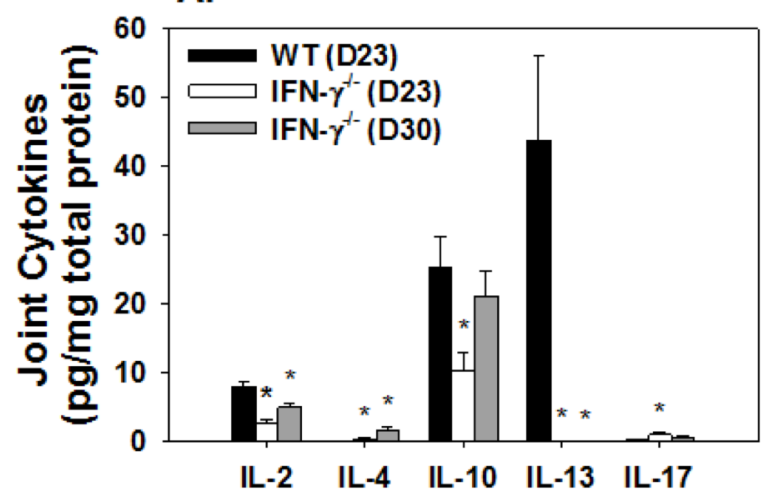

B.

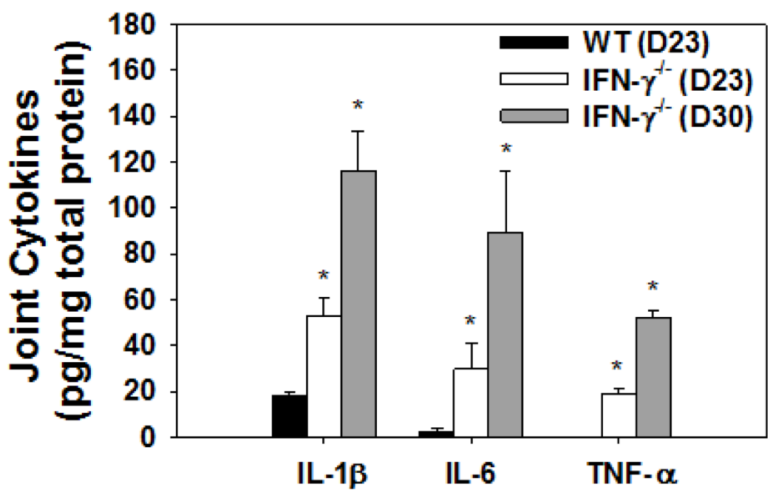

C.

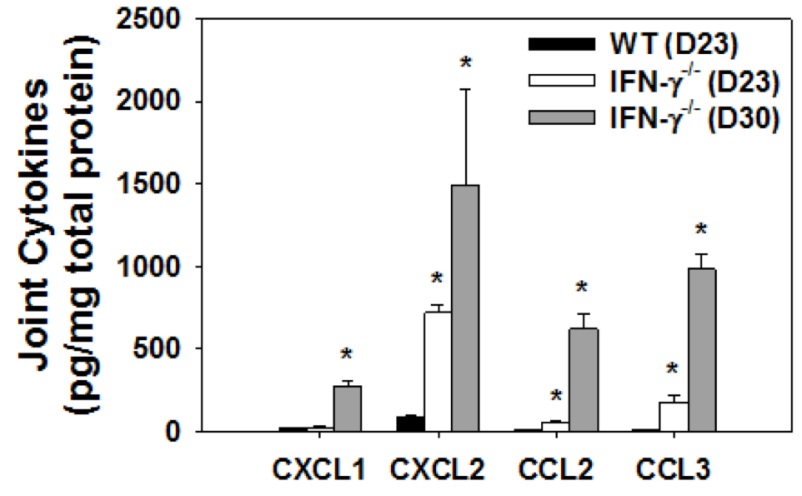

Figure 2. CCR2 and CXCR2 ligands are up-regulated in inflamed, $B$. melitensis-infected joints.

Joint homogenates ( $\mathrm{n}=5$ mice/group) from wild-type and IFN- $\gamma^{-/-}$mice infected i.p. with $\sim 1 \times 10^{5}$ CFUs $B$. melitensis $16 \mathrm{M}$ were assayed for T-cell-associated cytokines (A) inflammatory cytokines (B) and chemokines (C) by Luminex at 23 and 30 days post-infection and normalized by $\mathrm{BCA}$. ${ }^{*} \mathrm{P}<0.05$ as compared to WT mice. Error bars depicted SEM. 
increased on day 23 and 30 in IFN- $\gamma^{-/-}$versus WT mice, while CXCL1 was upregulated at day 30 post-infection (Fig 2C).

\section{CCR2 is not a critical mediator of Brucella-induced focal inflammation}

CCR2 is the primary receptor for CCL2 signaling and recruitment of inflammatory monocytes (168). CCL2 was elevated in joint homogenates from infected IFN- $\mathrm{Y}^{-1-}$ mice; therefore, we examined the role of CCR2 in Brucella-induced inflammation. Wild-type and CCR2 ${ }^{-/}$mice (C57BL/6 background) were treated with anti-IFN- $\gamma$, infected i.p. with B. melitensis and monitored over time. Incidence, clinical scores, and swelling were similar in wild-type and CCR2 ${ }^{-/}$mice Fig 3A-C). Additionally, tissue bacterial loads were comparable in $\mathrm{CCR}^{-/-}$and wild-type tissues (Fig 3D).

CXCR2 $^{--}$mice display reduced clinical signs of Brucella-induced inflammation

CXCL1 and CXCL2 were elevated in Brucella-infected joints and are both CXCR2 ligands that can recruit neutrophils to the site of infection (169). To elucidate the role of CXCR2 in Brucella-induced inflammation, wild-type BALB/C and $\mathrm{CXCR} 2^{-/}$mice (BALB/c background) were treated with anti-IFN-Y and infected i.p. with $\sim 1 \times 10^{5} \mathrm{CFUs}$ of $B$. melitensis. CXCR2 $2^{-/-}$mice displayed delayed incidence, reduced clinical scores and swelling compared to wild-type mice (Fig 4A-C). $\mathrm{CXCR}^{-/-}$mice did lose more weight than wild-type animals (data not shown), which may explain some of the decreased paw thickness in these mice (Fig 4C). 
A.

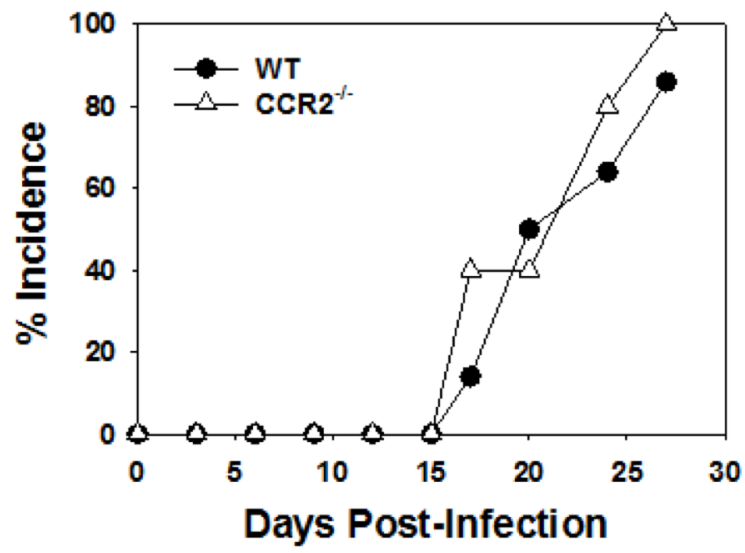

c.

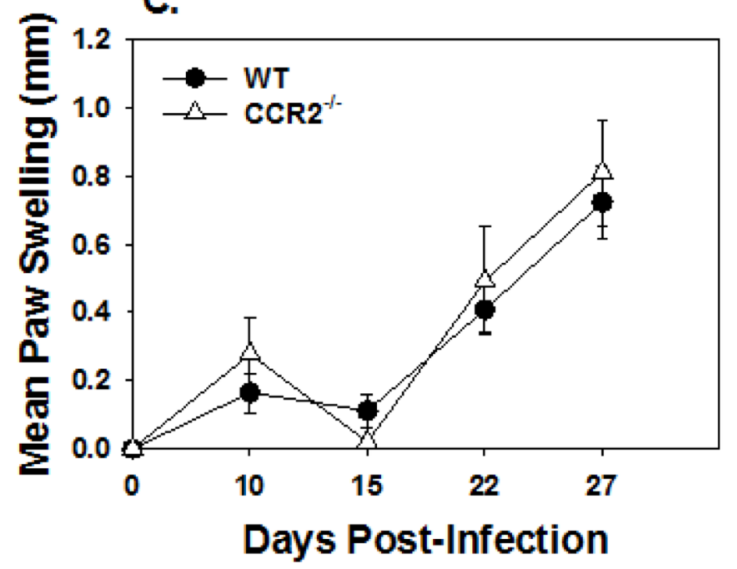

B.

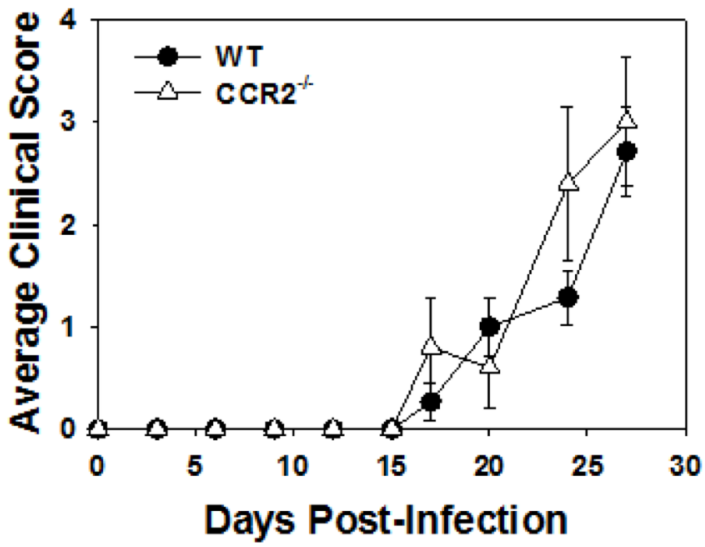

D.

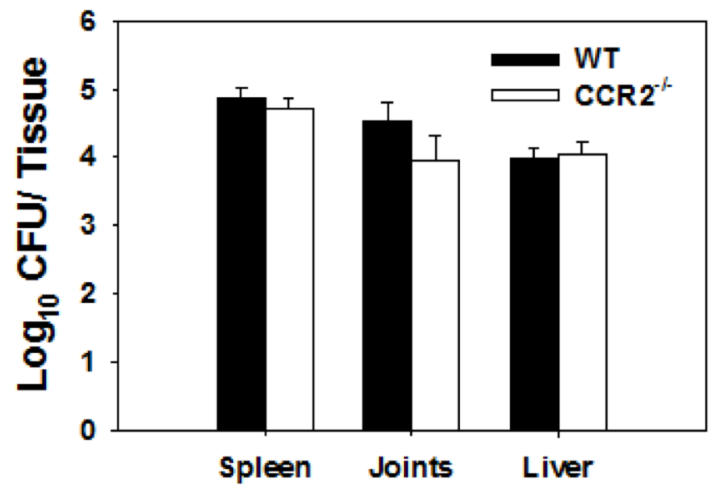

Figure 3. CCR2 is not a critical mediator of Brucella-induced focal inflammation.

Wild-type and CCR2 ${ }^{-/}$mice were infected i.p. with $\sim 1 \times 10^{5}$ CFUs of $B$. melitensis $16 \mathrm{M}$ and treated with anti-IFN- $\gamma(\mathrm{n}=5-10$ animals/group). Incidence of paw swelling (A), clinical scores (B), and mean swelling (C) were recorded over time. On day 28 post-infection mice were sacrificed and Brucella CFUs were enumerated (D). Error bars depict SEM. 
A.

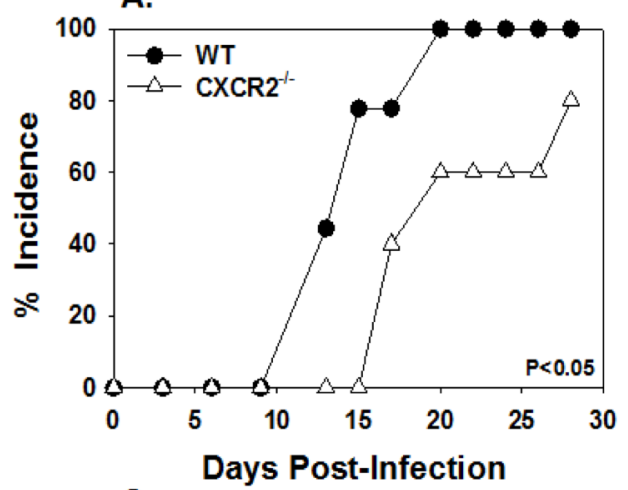

C.

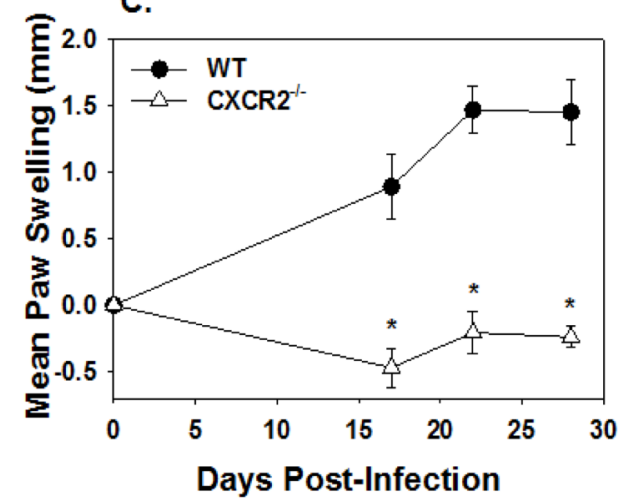

B.

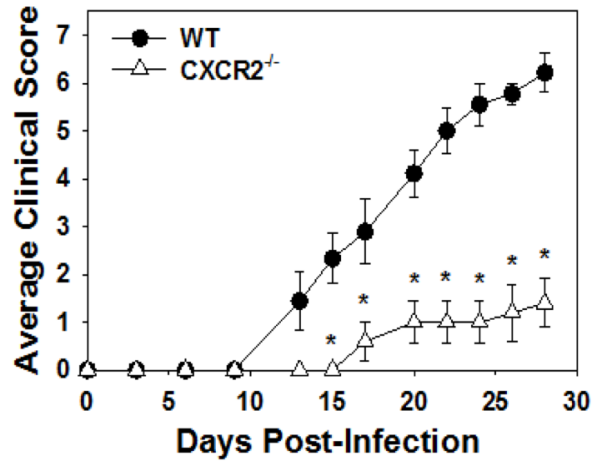

D.

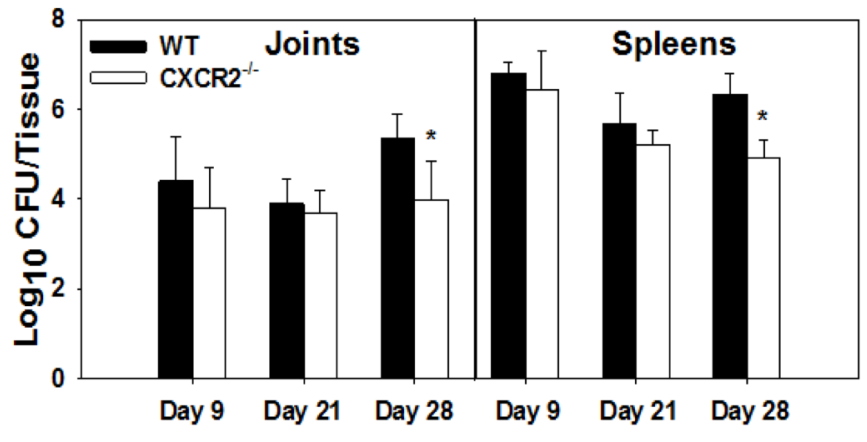

Figure 4. Reduced focal inflammation in Brucella- infected, anti-IFN-Ytreated, CXCR2 ${ }^{-/-}$mice.

Wild-type and $\mathrm{CXCR} 2^{-/-}$mice (both on a BALB/c background) were infected i.p. with $\sim 1 \times 10^{5}$ CFUs of $B$. melitensis $16 \mathrm{M}$ and treated with anti-IFN-Y. Incidence of paw swelling $(\mathbf{A})$, clinical scores $(\mathbf{B})$ and mean swelling $(\mathbf{C})$ were recorded over time. On days 9, 21 and 28 post-infection Brucella colonization was determined (D). ${ }^{*} \mathrm{P}<0.05$ as compared to WT mice. Error bars depict SEM. Data in A-C is from 5-10 mice/group and is representative of three independent experiments. CFU data in D) was obtained from 3-9 mice/aroun. Error bars depict SEM. 
Bacterial loads in the spleen and joints of wild-type and CXCR2 ${ }^{-/}$mice were similar at days 9 and 21 post-infection. However, at day 28, CXCR2 ${ }^{-/}$mice had significantly lower CFUs compared to wild-type mice in both the joints and spleen (Fig 4D). We also found that joints from CXCR2 ${ }^{-/}$mice had reduced levels of CCL2, IL-6, TNF- $\alpha$ and IL-1 $\beta$ compared to wild-type mice at 28 days post-infection (data not shown).

To confirm our results obtained by anti-IFN-y treatment, we generated IFN$\mathrm{Y}^{-1-} / \mathrm{CXCR} 2^{-1-}$ mice. Following infection with $B$. melitensis, IFN- $\mathrm{Y}^{-1-} / \mathrm{CXCR} 2^{-1-}$ mice had delayed arthritis onset with only $16.6 \%$ developing arthritis by day 19 compared to $100 \%$ of IFN- $\gamma^{-/-}$mice (Fig 5A). Additionally, IFN- $\gamma^{-1-} / \mathrm{CXCR} 2^{-/-}$mice had reduced clinical scores with an average score of 1 versus 8.87 for IFN- $\gamma^{-/-}$mice (Fig 5B). Swelling of the paws was also strikingly reduced in the IFN- $-\gamma^{-1-} / \mathrm{CXCR} 2^{-/-}$ mice (Fig 5C). IFN- $\gamma^{-1-} / \mathrm{CXCR}^{-/-}$mice did lose more weight compared to IFN- $\gamma^{-/-}$ mice; however, no significant change in bacterial loads in the spleen or joints between groups was noticed at days 18 and 25 post-infection (Fig 5D).

\section{CXCR2 mediates neutrophil infiltration in musculoskeletal tissue}

Gross swelling of mouse paws and tails was markedly reduced in IFN- $\gamma^{-1-}$ /CXCR2 $^{-/-}$mice relative to IFN- $\gamma^{-/-}$animals infected with B. melitensis (Fig 6A). Inflammation of mouse paws develops asymmetrically in Brucella-infected IFN- $\gamma^{-/-}$ mice, while tails typically develop multiple inflammatory foci (Fig 6A). Therefore to assess inflammation in the same tissue from all mice, histology was performed on mouse tails and scores were assigned as described in Methods. H\&E staining of 
A.

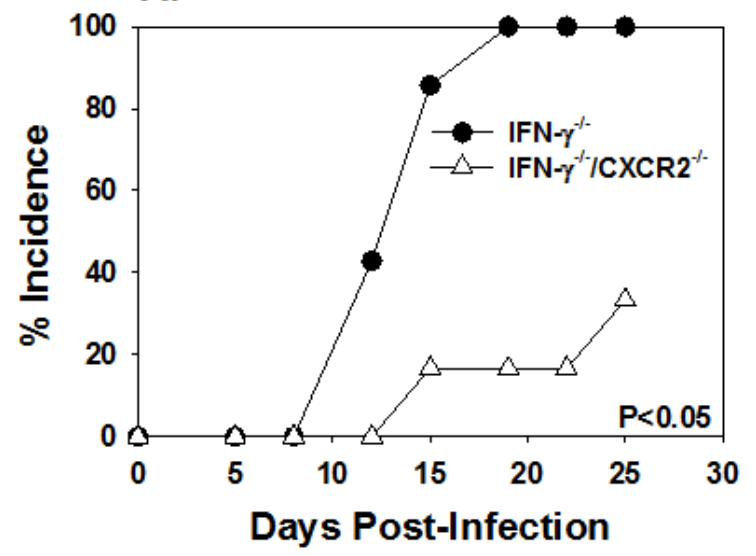

C.

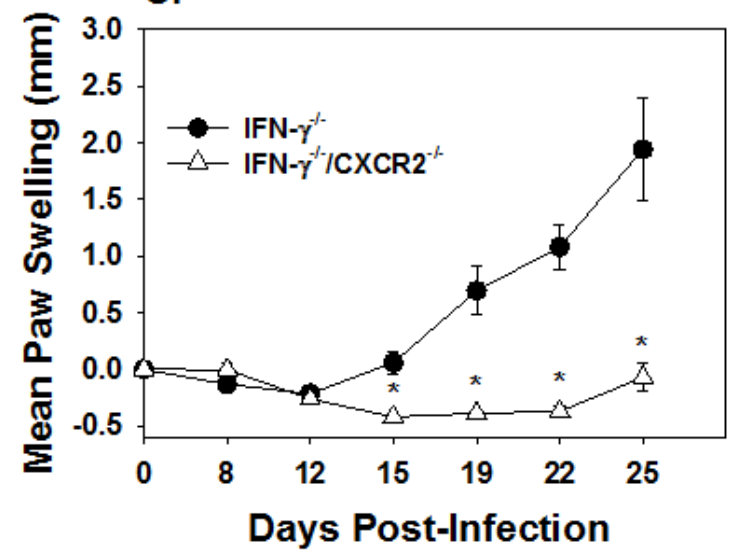

B.

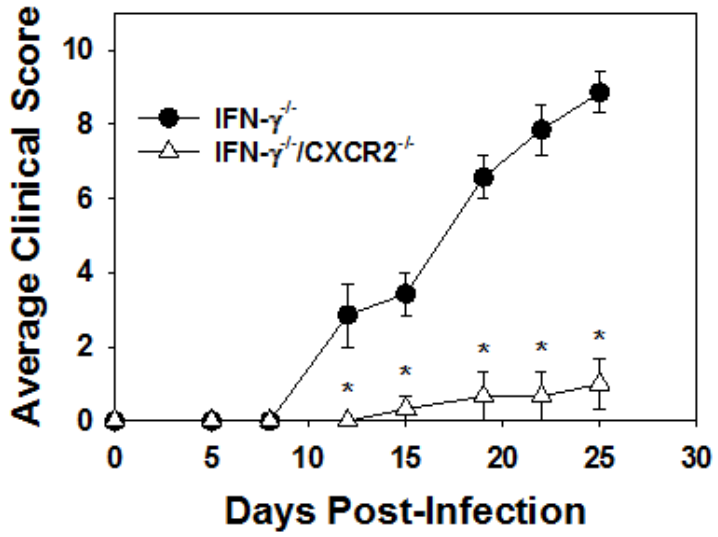

D.

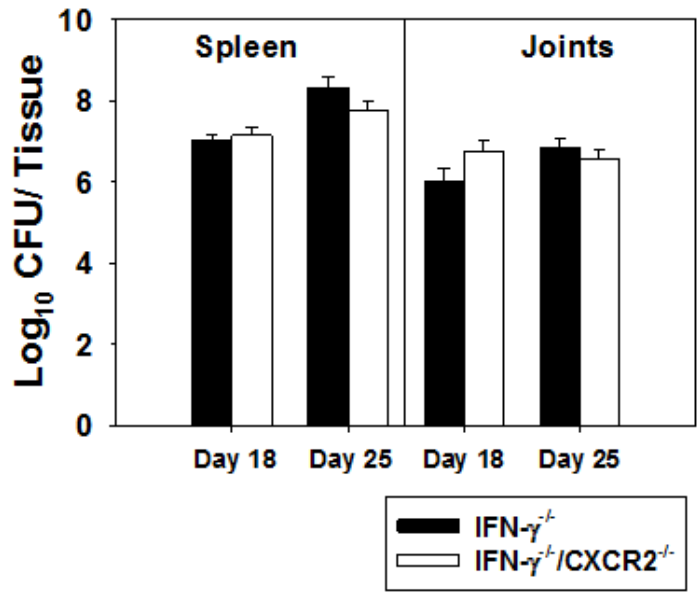

Figure 5. CXCR2 mediates Brucella-induced inflammation in IFN- $\gamma^{-/-}$mice. $\mathrm{IFN}-\mathrm{\gamma}^{-/-}$and IFN $-\gamma^{-/} / \mathrm{CXCR} 2^{-/-}$mice (on C57BL/6 background, $\mathrm{n}=4-7 /$ group) were infected i.p. with $\sim 1 \times 10^{5}$ CFUs $B$. melitensis. Incidence of paw swelling (A) clinical scores (B) and mean swelling (C) were recorded over time. Colonization of the joints and spleen was determined on day 18 and 25 post-infection (D). ${ }^{*} \mathrm{P}<0.05$ as compared to IFN- $\mathrm{F}^{-/}$mice. Data in (A-C) is representative of three independent experiments. Error bars depict SEM. 
A.

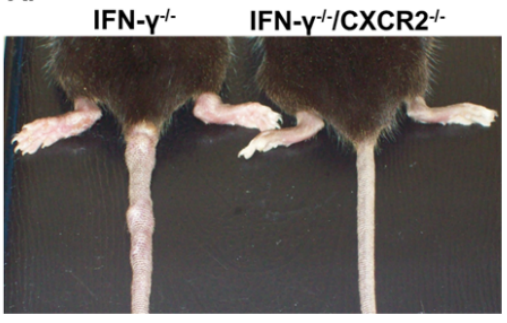

B.
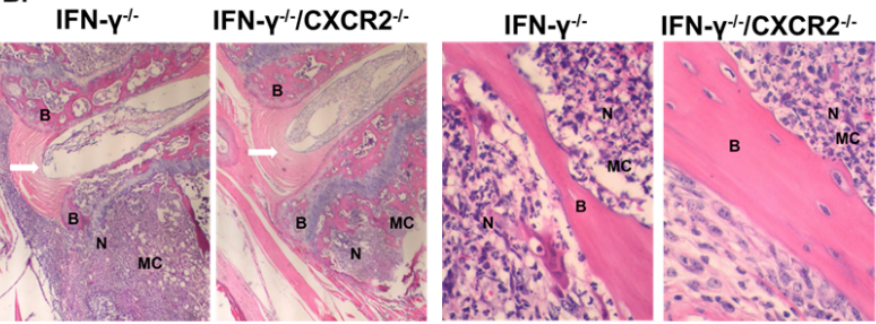

C. IFN- $\mathrm{Y}^{-1 .}$

IFN- $-\mathrm{Y}^{-1} / \mathrm{CXCR} 2^{-1-}$
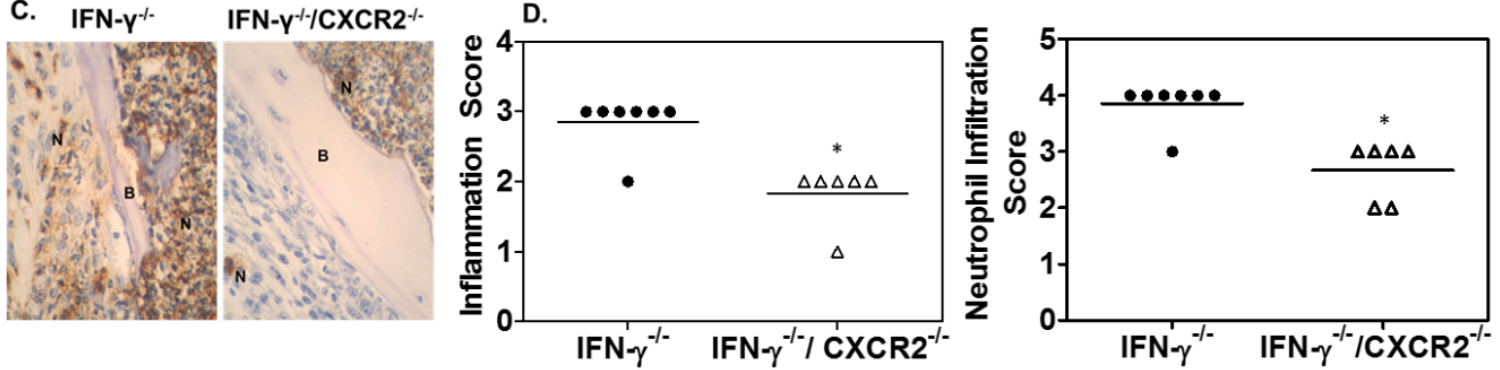

Figure 6. CXCR2 mediates musculoskeletal neutrophil recruitment in Brucella-infected mice.

IFN- $\gamma^{-/-}$and IFN- $\gamma^{-/ /} / \mathrm{CXCR}^{-/-}$mice were infected i.p. with $\sim 1 \times 10^{5} \mathrm{CFUs}$ of Brucella melitensis $16 \mathrm{M}$ and sacrificed at day 25 post-infection. Representative tail images comparing IFN- $\gamma^{-1 /}$ and IFN- $\gamma^{-1-} / \mathrm{CXCR}^{-1-}$ mice (A) Representative images of H\&E stained, B. melitensis -infected IFN- $\gamma^{-1-}$ and IFN- $\gamma^{-1-} / \mathrm{CXCR} 2^{-1-}$ tail vertebral sections using a $4 \mathrm{X}$ (left) and 40X (right) objective lens (B) Representative images of immunohistochemistry sections using anti-Ly6G, (clone 1A8) indicating neutrophil infiltration using a 40X objective (C) Scoring of histopathology H\&E (total inflammation) and immunohistochemistry (neutrophil infiltration) tail vertebra sections (6-7 mice/group) (D) (arrow) intervertebral disk, (B) bone, (MC) medullary cavity, (N) neutrophils ${ }^{*} \mathrm{P}<0.05$ as compared to sections from IFN- $\gamma^{-/-}$mice. 
mouse-tail sections from B. melitensis-infected IFN- $\gamma^{-/-}$mice revealed severe areas of inflammation that involved bone, medullary cavity of bone, tendons, skeletal muscle and periarticular inflammation (Fig 6B). Osteomyelitis was observed in the medullary cavity of $B$. melitensis-infected IFN- $\gamma^{-/-}$mice, which generally occurred near the growth plate, and in some instances may have led to erosion of the bone (Fig 6B). Osteomyelitis was also observed in $\mathrm{IFN}-\mathrm{Y}^{-1-} / \mathrm{CXCR} 2^{-/-}$mice infected with B. melitensis, but was much milder than that observed in IFN- $\gamma^{-1-}$ mice. Inflammation was sometimes localized to medullary cavity in IFN- $y^{-1 / C X C R 2} 2^{-1-}$ in contrast to IFN- $\gamma^{-/-}$mice that displayed extensive inflammation in bone, tendon, and skeletal muscle (Fig 6B\&D). Immunochemistry was also performed using the neutrophil specific Ly-6G antibody (clone 1A8). Neutrophil infiltration was seen in both groups, however IFN- $\gamma^{-/} / \mathrm{CXXR} 2^{-/-}$had significantly reduced neutrophil recruitment in inflamed tissues relative to $\mathrm{IFN}^{-\gamma^{-/}}$mice (Fig. 6C\& D). Flow cytometry also revealed a $\sim 40 \%$ reduction of neutrophil infiltration in joints from IFN- $\gamma^{-/-} / \mathrm{CXCR2} 2^{-/}$, relative to IFN- $\gamma^{-/-}$mice (data not shown).

\section{Reduced TNF- $\alpha$ and CCL3 levels in IFN- $\gamma^{-/-} /$CXCR2 $^{-/-}$mice}

Cytokines from Brucella-infected IFN- $\gamma^{-/-}$and IFN- $\gamma^{-/-} / \mathrm{CXCR}^{-{ }^{--}}$joints were evaluated by Luminex to determine signaling pathways that might be regulated by CXCR2. At day 25 post-infection, both TNF- $\alpha$ and CCL3 were significantly reduced in IFN- $\gamma^{-/} / \mathrm{CXCR}^{-/-}$compared to IFN- $\gamma^{-/}$joints (Fig 7A\&B). IL-1 $1 \beta$ levels were also lower, but not significantly in IFN- $\mathrm{Y}^{-1} / \mathrm{CXCR} 2^{-/-}$mice. Additionally, the anti- 

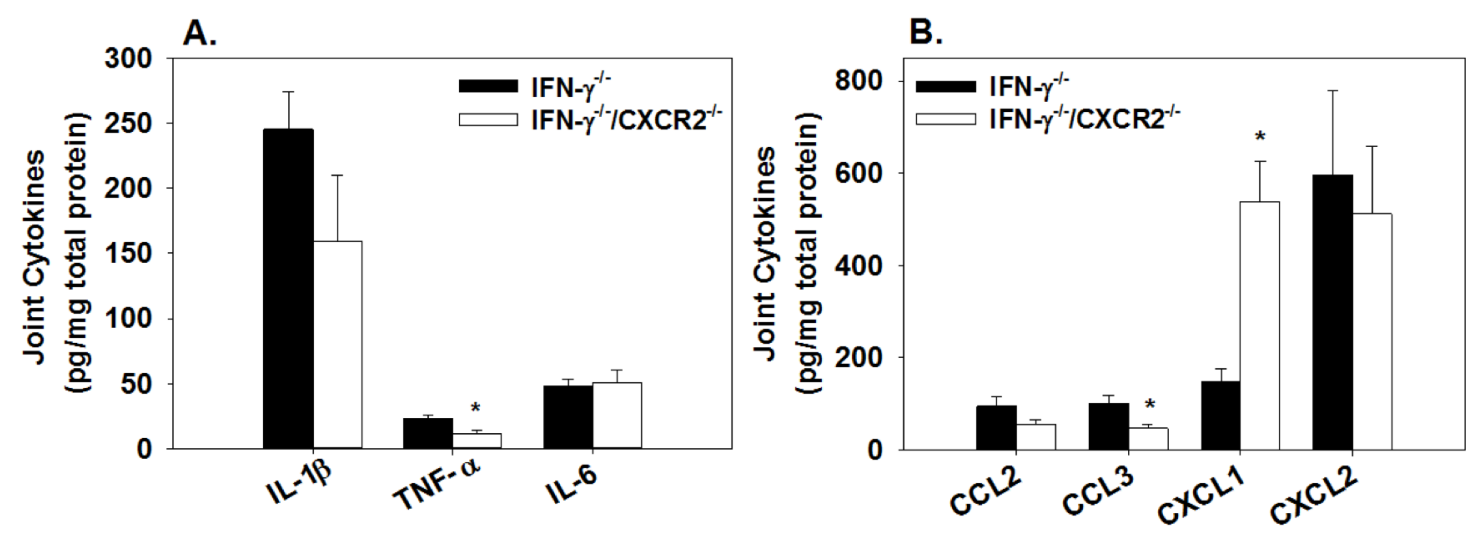

Figure 7. Reduced levels of TNF- $\alpha$ and CCL3 in IFN $-\gamma^{-1-}$ mice lacking CXCR2.

IFN- $\gamma^{-1-}$ and IFN- $\gamma^{-/} / \mathrm{CXCR} 2^{-/-}$mice were infected i.p. with $\sim 1 \times 10^{5} \mathrm{CFUs}$ of $B$. melitensis. Joint homogenates ( $n=6-7 /$ group) were assayed for inflammatory cytokines (A) and chemokines (B) at 25 days post-infection by Luminex and normalized by BCA. ${ }^{*} \mathrm{P}<0.05$ as compared to IFN- ${ }^{-1-}$ mice. Error bars depict SEM 
inflammatory cytokine IL-10 was slightly up-regulated in IFN- $\mathrm{y}^{-1-} / \mathrm{CXCR} 2^{-1-}$ mice on day 18 post-infection relative to IFN- $\gamma^{-1}$ mice (data not shown).

\section{DISCUSSION}

Brucellosis is one of the most common zoonotic diseases worldwide (8), and osteoarticular complications are the most frequent localized manifestations $(56,57)$. Here, we investigated mechanisms of inflammation during Brucella infection using our previously published mouse model (165). We found that T-and B-cell-deficient mice developed focal inflammation similarly to wild-type animals. We also did not observe a robust recruitment of $T$ and $B$ cells to infected joints. In contrast to rheumatoid arthritis, where auto-reactive $T$ cells and $B$ cells are the primary cause of pathogenesis $(170,171), T$ cell and antibody responses do not appear to be required for Brucella-induced inflammation, similar to what has been reported in other murine models of infectious arthritis, such as Lyme disease (172). While T and B cells were not required for the development of arthritis in our model, others have shown that Rag $1^{-/-}$mice are more susceptible to $B$. melitensis infection (173). B cell deficient mice are more resistant to Brucella infection (174); however, several other groups have shown that T cells mediate clearance of Brucella and are a major producer of IFN-ץ $(175,176)$. Thus, in future studies we will determine if transfer of wild-type T cells to IFN- $\gamma^{-/-}$mice can mediate resolution of Brucellainduced arthritis.

While adaptive immune cells are not required for Brucella-induced focal inflammation, we did find elevated levels of neutrophils in infected joints. CCR2, 
and its ligand CCL2 are typically involved in the recruitment of neutrophils and inflammatory monocytes $(128,177)$. In a model of antigen-induced arthritis, CCR2deficient mice developed significantly reduced arthritis and neutrophil infiltration within their joints, while administration of WT neutrophils to $\mathrm{CCR}^{-/-}$mice diminished protection (128). However, similar to what others have reported for Borrelia burgdorferi-induced arthritis (178), we did not find a prominent role for CCR2 in musculoskeletal brucellosis.

CXCR2 is important in neutrophil recruitment and subsequent antimicrobial functions including oxidative burst, degranulation, netosis, phagocytosis and is activated through the production of chemokines such as CXCL1 and CXCL2 (179, 180). Levels of CXCL1 and CXCL2 in Brucella-infected IFN- $\gamma^{-1-}$ joints correlated with the onset of inflammation. CXCR2 deficiency did not enhance susceptibility to infection as $B$. melitensis loads in the spleens and joints of $\mathrm{CXCR} 2^{--}$mice and control animals were similar. We also found that $\mathrm{CXCR} 2^{-/}$mice controlled Brucella infection as well as wild-type mice in the absence of anti-IFN-y treatment (data not shown). Therefore, CXCR2, under these conditions, was not required for the clearance of Brucella. Others have demonstrated that during chronic infection, neutrophils impede clearance of $B$. abortus, perhaps by reducing Th1 responses (181). Thus, reduced neutrophil recruitment in CXCR2-deficient animals is not likely to result in enhanced susceptibility to Brucella infection.

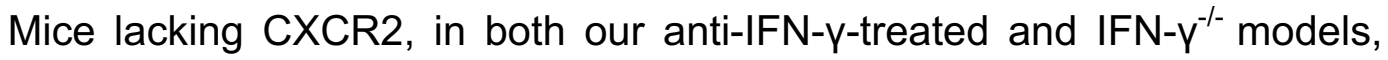
displayed markedly reduced symptoms of focal inflammation relative to control animals. These findings may be clinically relevant as elevated levels of CXCL8, 
a human chemokine that signals through CXCR2, were found in the synovial fluid of a human articular brucellosis patient (182). CXCR2 was required for neutrophil recruitment to Brucella-infected joints. Studies using collagen-induced, K/BXN, and LPS-induced models of arthritis suggest neutrophil depletion using anti-Gr1 antibodies is protective against disease $(183,184)$. However in experimental Lyme arthritis, neutrophil depletion, with RB6-8C5 antibody, results in early induction of arthritis and infiltration of PMN/leukocyte-like cells that do not express Gr-1 (185). Here we found that, CXCR2-deficient animals had reduced levels of CCL3 and TNF- $\alpha$, compared to control animals. Some evidence suggests neutrophil recruitment can be dependent on CXCL2 inducing the release of CCL3 with subsequent induction of TNF- $\alpha$ (186). Future studies will determine the role of neutrophils and the signaling pathways that recruit them in Brucella-induced focal inflammation.

The signaling pathways and cells that produce the CXCR2 ligands, CXCL1 and CXCL2, have yet to be determined in our murine model, however it has been demonstrated that TLR2 is required for alveolar macrophages to produce CXCL1 in response to $B$. abortus infection (108). In addition to professional phagocytes, cells native to the joint may also be producing CXCR2 ligands. One study showed that when human fibroblast-like synoviocytes are infected with $B$. abortus they produce IL-6, CCL2, and CXCL8, in a dose-dependent manner. Additionally, these supernatants recruited human PMNs and monocytes in vitro (187). Furthermore, human osteoblasts up-regulate their production of CCL2, IL-6 and CXCL8 upon co-culture with supernatant from $B$. abortus-infected monocytes. In turn, media 
conditioned by $B$. abortus-infected osteoblasts induced monocyte production of CXCL8, TNF- $\alpha$, IL-6 and IL-1 $\beta$ (129).

Collectively, our study suggests blocking or inhibition of CXCR2 may be a potential therapy for treating Brucella-induced inflammation. Genetic ablation of CXCR2 resulted in reduced arthritis in a mouse model of Lyme disease $(178,188)$. Similarly, blocking CXCR2 through various techniques has proven to be therapeutic in treating models of rheumatoid arthritis (189-191). In addition, treating mice with allosteric inhibitors of CXCR1 and CXCR2 reduced the number of neutrophils in the synovium in a mouse model of antigen-induced arthritis (192). Therefore, the targeting of CXCR2, or CXCR2 ligands, may have potential as a complementary therapy, in combination with antibiotics, for treating osteoarticular complications of brucellosis. 


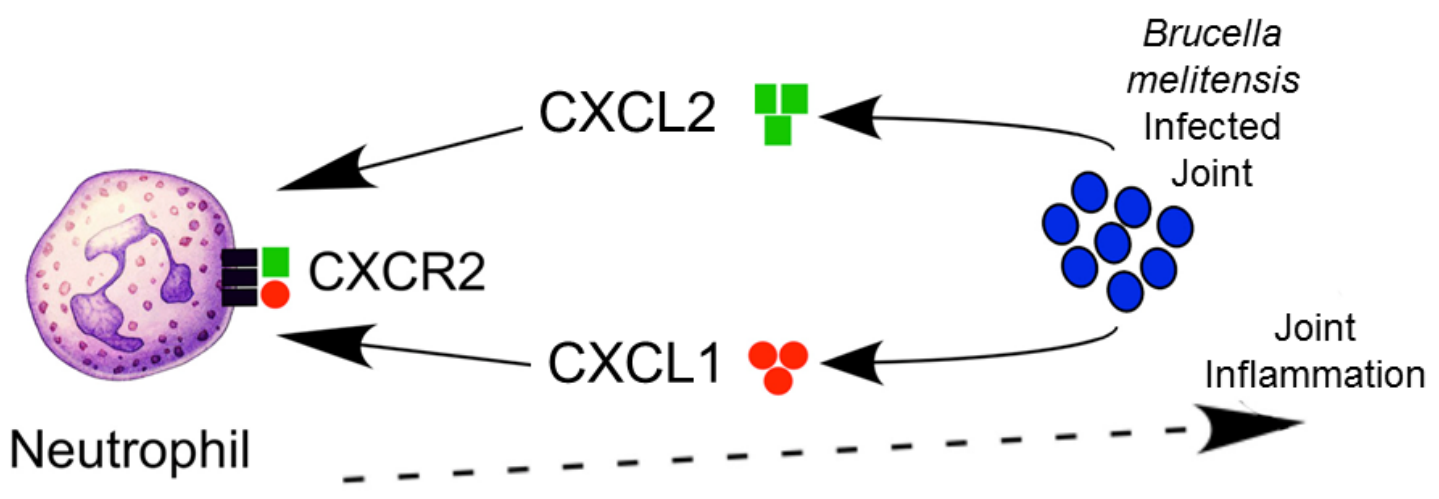

Figure 8. Model of Brucella Induced Arthritis in i.p. infected IFN- $\gamma$ deficient mice.

Brucella infects the joint space and local tissues produce CXCL1 and/or CXCL2. The neutrophil CXCR2 receptor is activated by ligands CXCL1 and CXCL2. Thus, neutrophils become activated and are recruited to the joint. 


\section{CHAPTER IV}

\section{Temporal Role for MyD88 in a Model of Brucella-Induced Arthritis and Musculoskeletal Inflammation}

\section{ABSTRACT}

Brucella spp. are facultative intracellular Gram-negative bacteria that cause the zoonotic disease brucellosis, one of the most common global zoonoses. Osteomyelitis, arthritis and musculoskeletal inflammation are common focal complications of brucellosis in humans; however, wild-type (WT) mice infected systemically with conventional doses of Brucella do not develop these complications. Here we report WT C57BL/6 mice infected via the footpad with $10^{3}$ $10^{6}$ CFUs of Brucella spp. display neutrophil and monocyte infiltration to the joint space and surrounding musculoskeletal tissue. Joint inflammation is detectable as early as one day post-infection and peaks one to two weeks later after which WT mice are able to slowly resolve inflammation. $\mathrm{B}$ and $\mathrm{T}$ cells were dispensable for the onset of swelling but required for resolution of joint inflammation and infection. At early time points, MyD88 ${ }^{-/-}$mice display decreased joint inflammation, swelling, and pro-inflammatory cytokine levels relative to WT mice. Subsequently, swelling of MyD88 $8^{-/}$joints surpassed WT joint swelling, and resolution of joint inflammation was prolonged. Joint bacterial loads in $\mathrm{MyD}^{-{ }^{--}}$mice were significantly greater than WT by day 3 post-infection and at all time points thereafter. In addition, MyD88 $^{-1-}$ joint inflammatory cytokine levels on day 3 and beyond were similar to WT. Collectively this data demonstrates MyD88 signaling mediates early inflammatory responses in the joint, but also contributes to subsequent clearance 
of Brucella and resolution of inflammation. This work also establishes a mouse model for studying Brucella-induced arthritis, musculoskeletal complications, and systemic responses, which will lead to a better understanding of focal complications of brucellosis.

\section{INTRODUCTION}

Mechanisms underlying arthritic responses induced by Brucella have been hindered by the lack of relevant mouse models, as wild-type mice infected with conventional doses $\left(10^{4}-10^{6}\right.$ colony forming units [CFUs]) by systemic routes do not develop joint inflammation $(58,70)$. One study showed that BALB/c mice infected intraperitoneally (i.p.) with $1 \times 10^{7} \mathrm{CFUs}$ of $B$. melitensis developed arthritis at 26 weeks post-infection (75). Additionally, others have previously reported that i.p. Brucella infection of IFN- $\gamma$-deficient mice resulted in arthritis of ankle joints as early as 15 days post-infection. Arthritis in this model was dependent on CXCR2 and IL-1R, but adaptive immune cells were not required for the induction of inflammation $(58,70)$. We suspected that IFN- $\gamma$ deficiency allowed for the hematogenous spread of Brucella, resulting in infection and inflammation of the joint (58). Other mouse models of infectious arthritis have utilized footpad inoculation to localize pathogens, such as Borrelia burgdorferi, Chikungunya virus, and Candida albicans, to the joint and surrounding tissue to induce inflammation $(172,193,194)$ Here we describe a model of Brucella-induced arthritis that is applicable in WT mice. Infection of the articular space and surrounding tissue is achieved by injecting Brucella adjacent to ankle joints, via footpad inoculation. 
Myeloid differentiation factor 88 (MyD88) is an adaptor protein that relays Toll-like receptor (TLR), IL-1R and IL-18R signaling (195, 196). Once activated, most TLRs signal through MyD88 to induce cytokine production (113). This signaling can result in arthritis and osteoclastogenesis in murine models of bacterial-induced focal complications (114-116). Host cells reportedly recognize Brucella through TLR2, TLR4, TLR6 and TLR9 (108-112). To determine if MyD88 signaling is important for Brucella-induced arthritis, we investigated joint inflammatory responses in MyD88 ${ }^{-/-}$mice following footpad infection with Brucella. We show MyD88 regulates early arthritis development, but is also required to control bacterial burden and resolve inflammation at later stages of infection.

\section{RESULTS}

\section{Brucella infection of the footpad results in arthritis and musculoskeletal inflammation in wild-type mice.}

The systemic spread of Brucella to the joints is thought to induce arthritis and musculoskeletal inflammation (58). Thus, we hypothesized that delivering Brucella adjacent to the joint would allow for bacterial colonization and subsequent arthritis. Therefore, we infected wild-type C57BL/6 mice (WT) in the rear footpads with $1 \times 10^{6} \mathrm{CFUs}$ of $B$. melitensis $16 \mathrm{M}$. H\&E staining was conducted on mockinfected mice at day 3, and Brucella-infected mice at days 1, 3, 7, 14 and 28 postinfection (Fig. 9A-F). All footpad-infected mice exhibited signs of arthritis and musculoskeletal inflammation in the joint and surrounding muscle and soft tissues 
A.

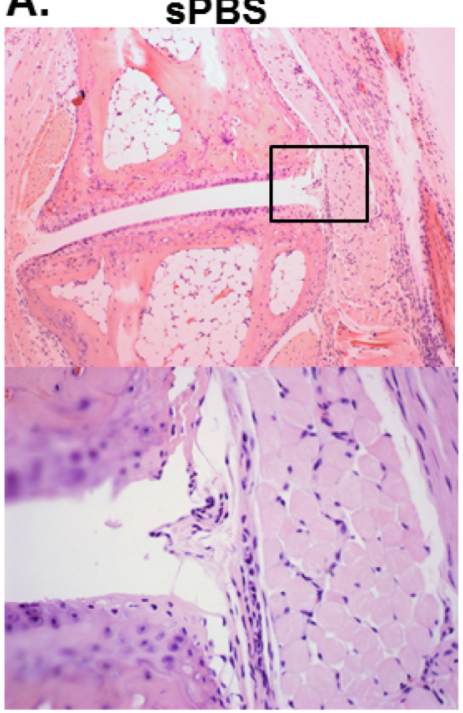

D.

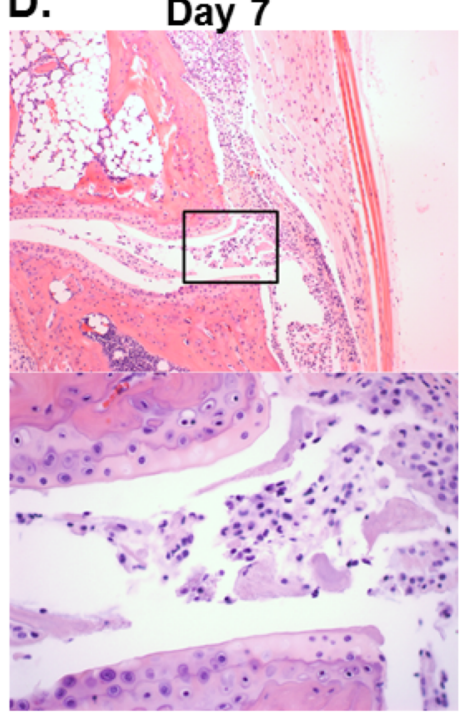

B.

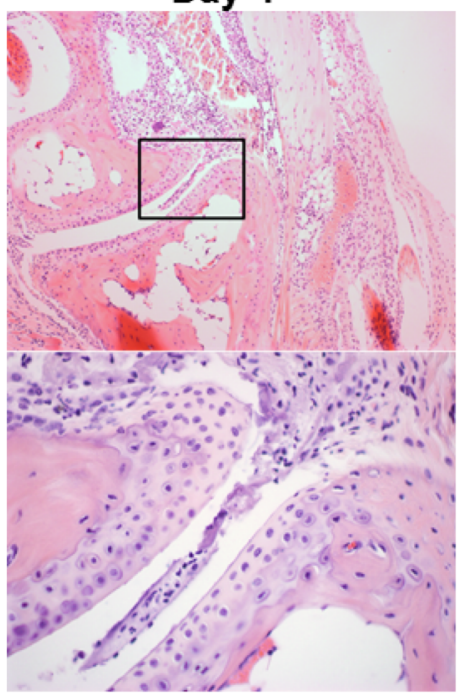

E.

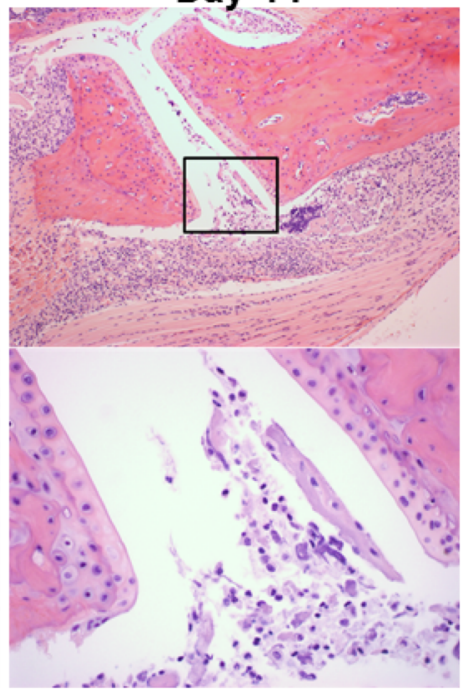

C. Day 3

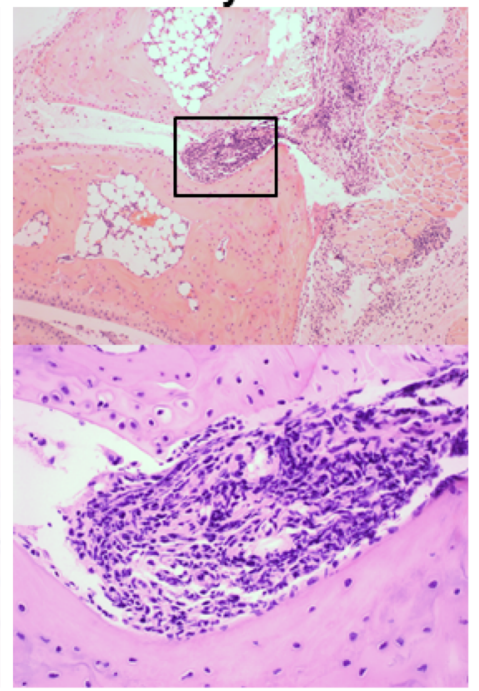

F.

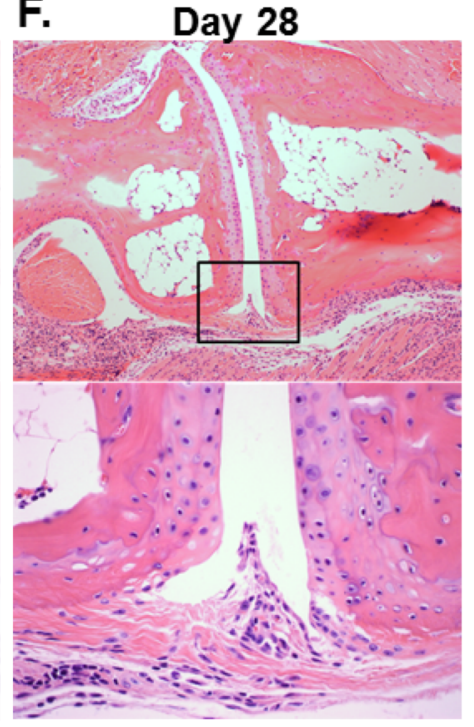

Figure 9. WT mice develop arthritis and musculoskeletal inflammation following footpad infection.

WT, C57BL/6, mice were either mock-infected in each rear footpad with sterile PBS (sPBS) or infected with $1 \times 10^{6}$ B. melitensis $16 \mathrm{M}$. Mock-infected mice were euthanized at day $3(\mathbf{A})$, and $B$. melitensis -infected mice at days 1 (B), 3 (C), 7 (D), 14 (E) and 28 (F) post-infection. H\&E staining was conducted on mouse joints. Representative images (100x) from mock treated, and infected mice are depicted. Amplified boxed regions (400x) showing inflammation within the joint space of $B$. melitensis-infected mice are displayed beneath the 100x image at each timepoint. Images are representative of joints from 4 to 5 mice per timepoint from one kinetic experiment. Similar pathology was observed at the day 3 and day 28 timepoints in 2-3 additional experiments. 
relative to mock-infected mice (Fig. 9B-F). Brucella-infected C57BL/6 mice demonstrated moderate to focally extensive areas of inflammation and large numbers of neutrophils, including neutrophils in the joint space, one day after infection (Fig. 9B). Joint arthritis and musculoskeletal inflammation peaked from days $3-14$, presenting with severe large confluent areas of inflammation (neutrophils, macrophages and fibrin in the joint space) (Fig. 9C-E). Inflammation began to resolve by day 28 , with moderate to severe pathology with focally extensive areas of inflammation including macrophages, fibrin and few neutrophils in the joint space (Fig. 9F).

\section{Footpad inoculation of mice with B. melitensis, B. suis or B. abortus results} in dose dependent joint swelling, arthritis and musculoskeletal inflammation.

To determine if footpad inoculation with a lower dose or other Brucella species pathogenic in humans could also induce arthritis, we injected $1 \times 10^{5} \mathrm{~B}$. melitensis $16 \mathrm{M}, B$. suis 1330 , or $B$. abortus 2308 into the footpads of wild-type C57BL/6 (WT) mice. Joint swelling was also measured as it is a characteristic manifestation of Brucella-induced arthritis and musculoskeletal inflammation (58, 70). Relative to B. melitensis-infected mice, swelling of joints was slightly, but significantly, greater in B. abortus-infected mice on day 2 , and in $B$. suis-infected mice on day 2 and 11. However, all Brucella species displayed similar swelling kinetics and induced robust swelling of mouse ankles by day 2 post-infection (Fig. 10A). Additionally, we investigated if $B$. melitensis $16 \mathrm{M}$ at a dose of $1 \times 10^{3}$ or $1 \times 10^{4}$ 

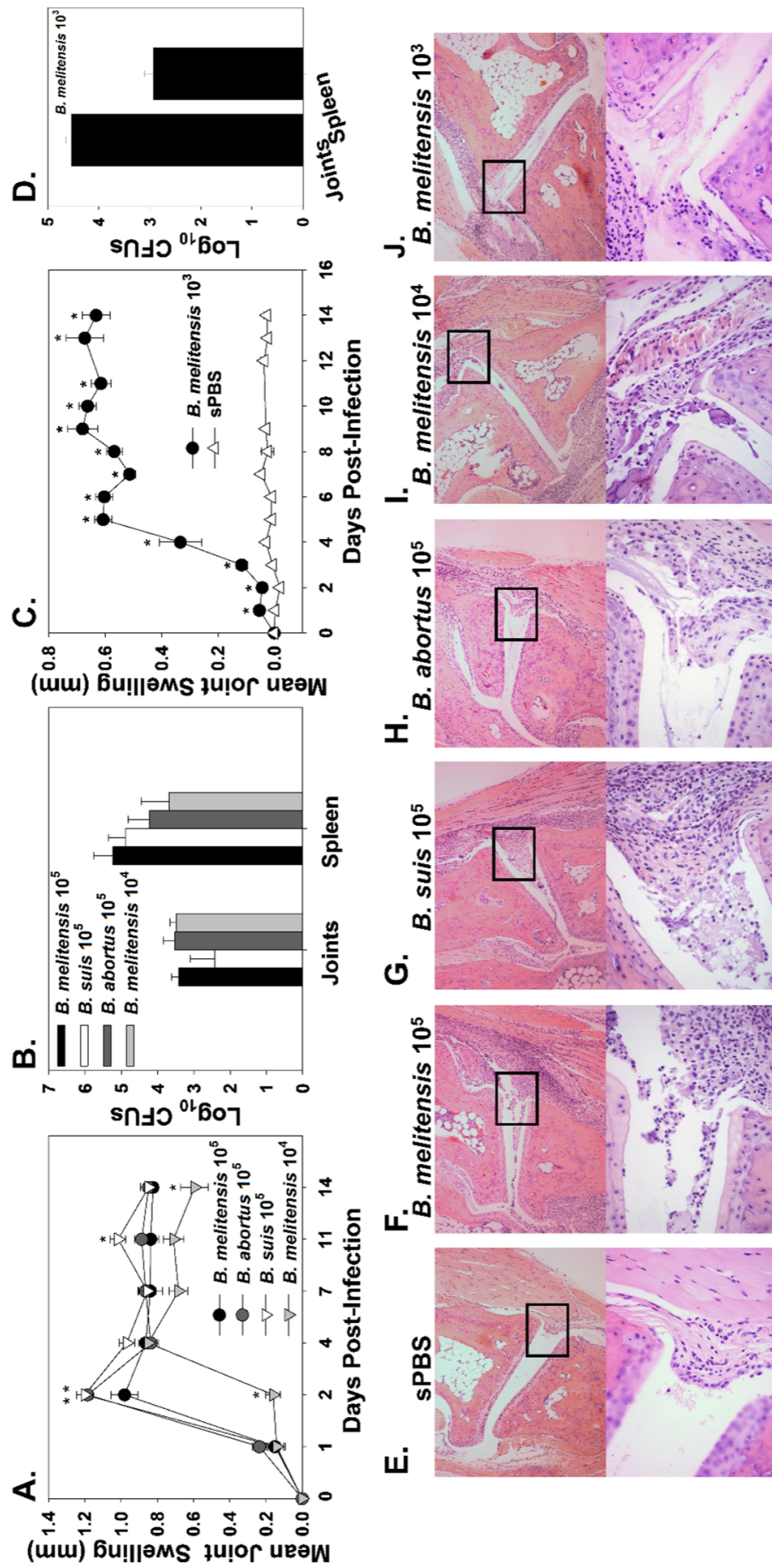

ய

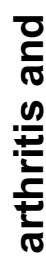

응

d

(n)

5

क

o

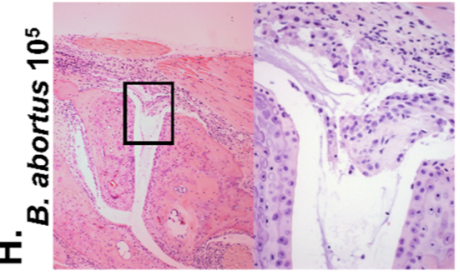

(ن)
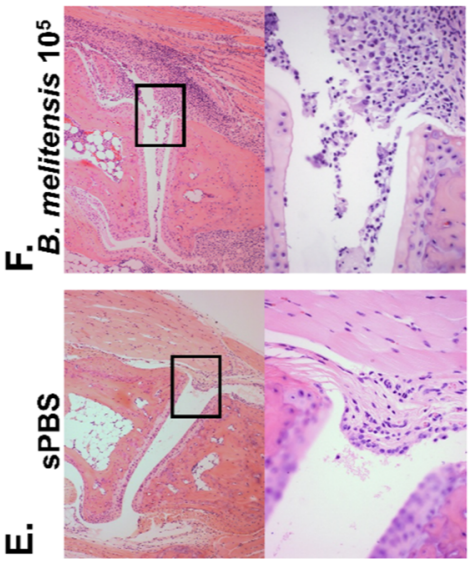

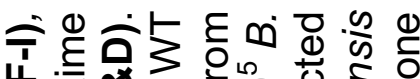

4.

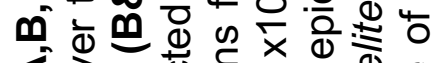

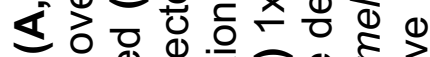

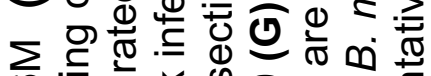

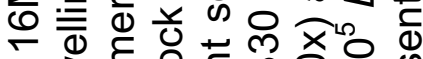
娄 क人 ब잉

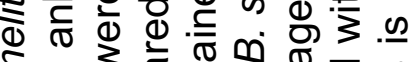
$\varepsilon \div 3 \pi \frac{\pi}{\omega} \infty \pi$ ต 능 응 는

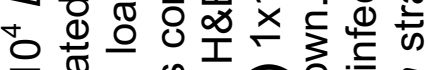

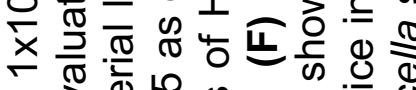

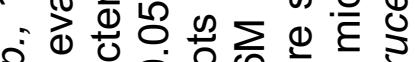

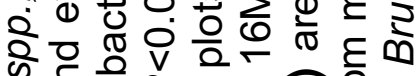

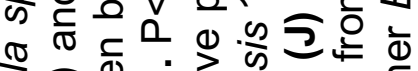
बे

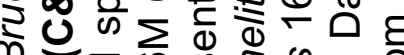

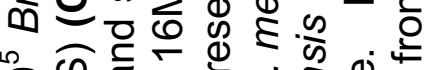
ம0 की

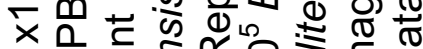
勿

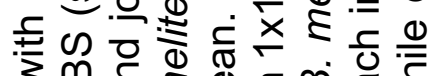

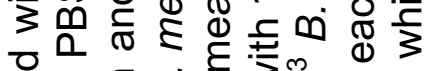
西

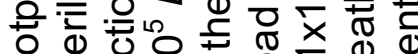
는

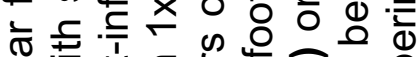

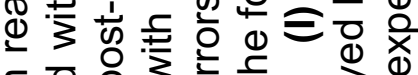

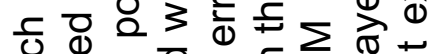
过 (1) .. वे 응 $\therefore$ 웡워

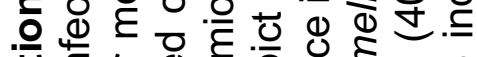

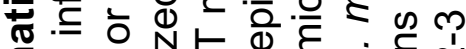

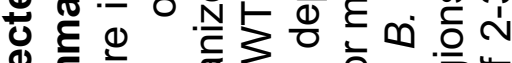
ब ह

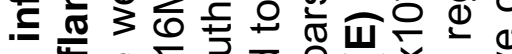
ฮั.

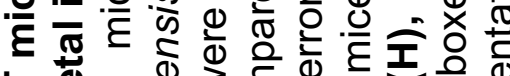

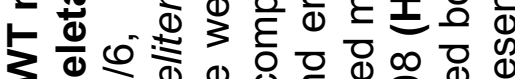

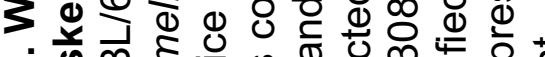

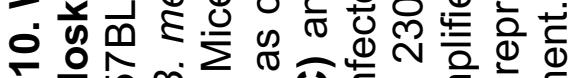

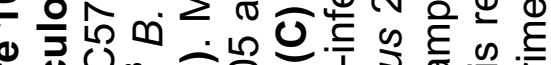

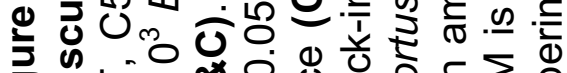

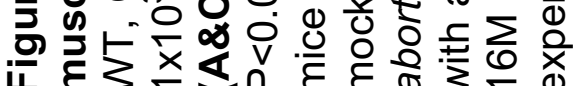


could induce inflammation via footpad infection (Fig 10A\&C). Although ankle swelling was evident, it was slightly delayed in mice infected with a dose of $1 \times 10^{4}$ CFUs when compared to mice infected with $1 \times 10^{5}$ B. melitensis (Fig. 10A). Similarly, mice infected with $1 \times 10^{3}$ B. melitensis also developed joint swelling, while no apparent swelling was in mice mock-infected with sterile PBS (Fig. 10C). Additionally, bacterial loads in joint homogenates were similar between mice infected with $1 \times 10^{5}$ CFUs of $B$. abortus, B. suis, or B. melitensis 14 days after infection (Fig. 10B). To determine if Brucella was able to colonize systemic tissues in this model, splenic bacterial loads were measured and B. melitensis, B. abortus, and $B$. suis were all found to similarly colonize the spleen following footpad inoculation with $1 \times 10^{5}$ CFUs. (Fig. 10B). Bacterial colonization was also detected in the joints and spleens of mice infected with $1 \times 10^{3}$ or $1 \times 10^{4}$ B. melitensis (Fig. 10B\&D). On day 14 post-infection, histology was conducted on joint sections from mock-infected mice, along with mice infected with $1 \times 10^{3}-1 \times 10^{5} \mathrm{~B}$. melitensis or with $1 \times 10^{5}$ B. suis or B. abortus (Fig. 10E-J). All Brucella-infected mice (Fig. 10FJ) developed arthritis and musculoskeletal inflammation while no pathology was evident in sterile PBS-inoculated animals (Fig. 10E). All mice infected with $1 \times 10^{5}$ Brucella spp. had a similar degree of neutrophil and macrophage infiltration in and around the joint space (Fig. 10F-H). Mice infected with lower doses of $B$. melitensis (Fig. 10I\&J) also developed arthritis and musculoskeletal inflammation. While doses of $1 \times 10^{3}-1 \times 10^{6} \mathrm{CFUs}$ all induced inflammation in this model, a dose of $1 \times 10^{6}$ was chosen for future studies as it was found to reliably generate detectable levels of joint cytokines in pilot studies (data not shown). 
Adaptive immune responses mediate resolution of inflammation, but are not required for development of joint swelling.

To investigate the role of adaptive immune responses during focal inflammation, wild-type C57BL/6 (WT) and $\mathrm{Rag}^{1 /-}$ (B and T cell deficient) mice were infected in each hind footpad with $1 \times 10^{6} \mathrm{CFUs}$ of $B$. melitensis $16 \mathrm{M}$, and ankle swelling was followed for 30 days post-infection. At days 1 and 3 postinfection both WT and Rag $1^{-/-}$mice had developed a similar degree of ankle swelling; however, at days 6 and $8 \operatorname{Rag} 1^{-/}$mice displayed a slight, but statistically significant, decrease in swelling compared to WT mice. After day 8 WT mice began to resolve joint swelling, but in Rag $1^{-/-}$mice swelling remained constant and was significantly greater than that present in WT mice by day 27 post-infection (Fig. 11A). Joint Brucella loads in Rag $1^{-/}$mice were more than 1,000 fold greater than those in WT mice by day 30 post-infection (Fig. 11B). Joint inflammation was higher at day 30 in $R a g 1^{-1-}$, relative to WT mice and joint levels of the proinflammatory cytokines CXCL1, IL-1 $\beta, C C L 2$, and IL-6 were also significantly greater in Rag $1^{-/-}$mice compared to WT mice (Fig. 11C-E). In addition, joints from WT mice had reduced amounts of fibrin relative to joints from Rag $1^{-/-}$mice (Fig. 11E). These data demonstrate that adaptive immunity contributes to, but is not absolutely required for, the initiation of joint swelling, and subsequently mediates bacterial clearance and resolution of joint inflammation. 
A.

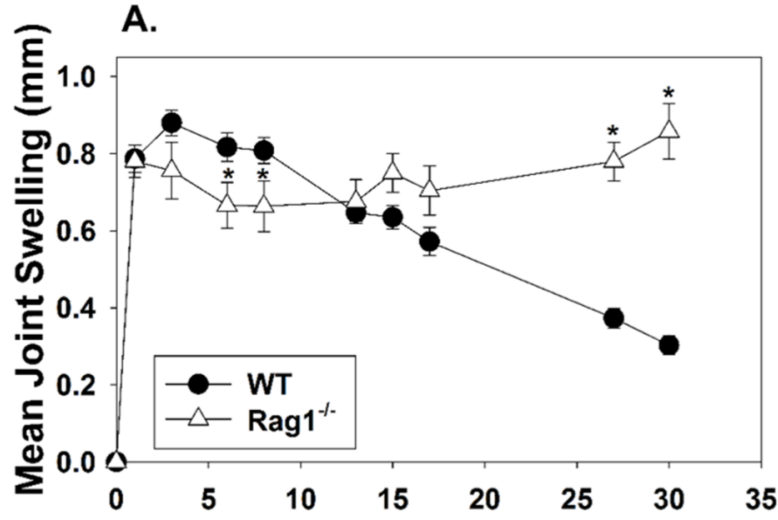

D. Days Post-Infection

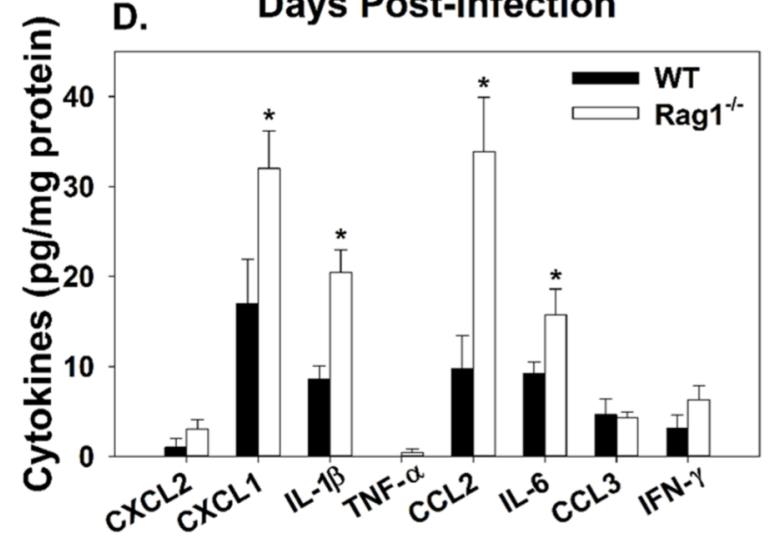

B.

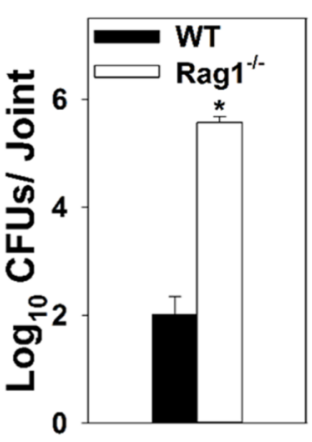

E.

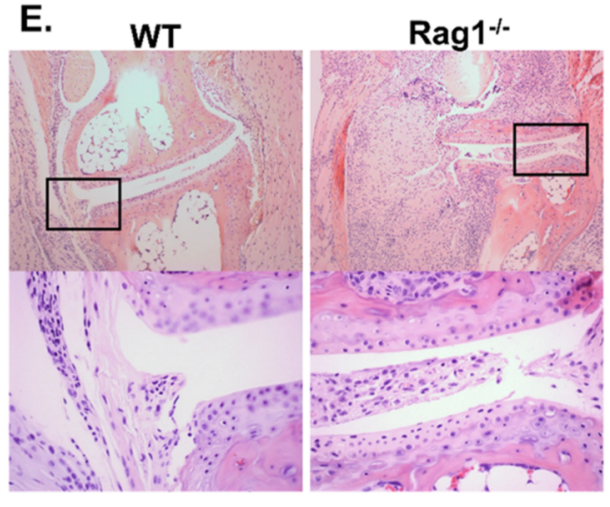

Figure 11. Adaptive immune responses are needed for inflammation resolution but not required for inflammation onset.

WT, C57BL/6, and Rag $1^{-1-}$ mice were infected in each rear footpad with $1 \times 10^{6}$ B. melitensis 16M. Joint swelling was recorded over time (5-10 mice/group) (A). Mice were euthanized at day 30 post-infection and ankle joint bacterial loads ( $n=5-10$ group) (B) and cytokine levels ( $n=4-5$ mice/group) (D) were enumerated. H\&E staining was conducted on joints at day 30 post-infection ( $n=5-6 /$ group) and scored for inflammation severity (C\&E). Representative images are depicted (100x) and amplified boxed regions are displayed beneath each image (400x) (E). $\quad P<0.05$ as compared to WT mice. Error bars depict standard errors of the mean. Data is representative of one experiment. 
MyD88 initiates early joint swelling, but is also required for clearance of Brucella from the joint and resolution of joint swelling

To determine if MyD88 was involved in the initiation of joint inflammation, swelling of wild-type C57BL/6 (WT) and MyD88 ${ }^{-/-}$mouse ankles was measured over 28 days post Brucella-infection. MyD88 ${ }^{-/-}$mice infected with $1 \times 10^{6} \mathrm{~B}$. melitensis $16 \mathrm{M}$ displayed reduced ankle swelling at days 1 and 3 post-infection (Fig. 12A). In contrast to the phenotype seen at day 1 and 3, MyD88 $^{-/-}$joints had increased swelling compared to WT joints by day 7 and at all subsequent timepoints post-infection (Fig. 12A). A similar effect of MyD88 on joint swelling was observed (albeit with delayed kinetics), when an infectious dose of $1 \times 10^{5}$ CFUs was used (Fig. 12B).

To explain the increase in swelling of MyD88 ${ }^{-/}$joints after day 3 postinfection, we hypothesized that MyD88 is important for mediating bacterial clearance in the joint. Thus, bacterial loads were examined in ankle homogenates on days $1,3,7,14$ and 28 post-infection with $1 \times 10^{6}$ B. melitensis (Fig. 12C). While joint CFUs were similar between groups at day 1 , by day 3 there were significantly greater bacterial loads in MyD88 ${ }^{-1-}$ joints when compared to WT mice. This trend continued throughout the experimental time course (Fig. 12C).

\section{MyD88 initiates early recruitment of inflammatory infiltrates following $B$. melitensis footpad infection.}

To assess the role of MyD88 in joint inflammation, H\&E staining was 

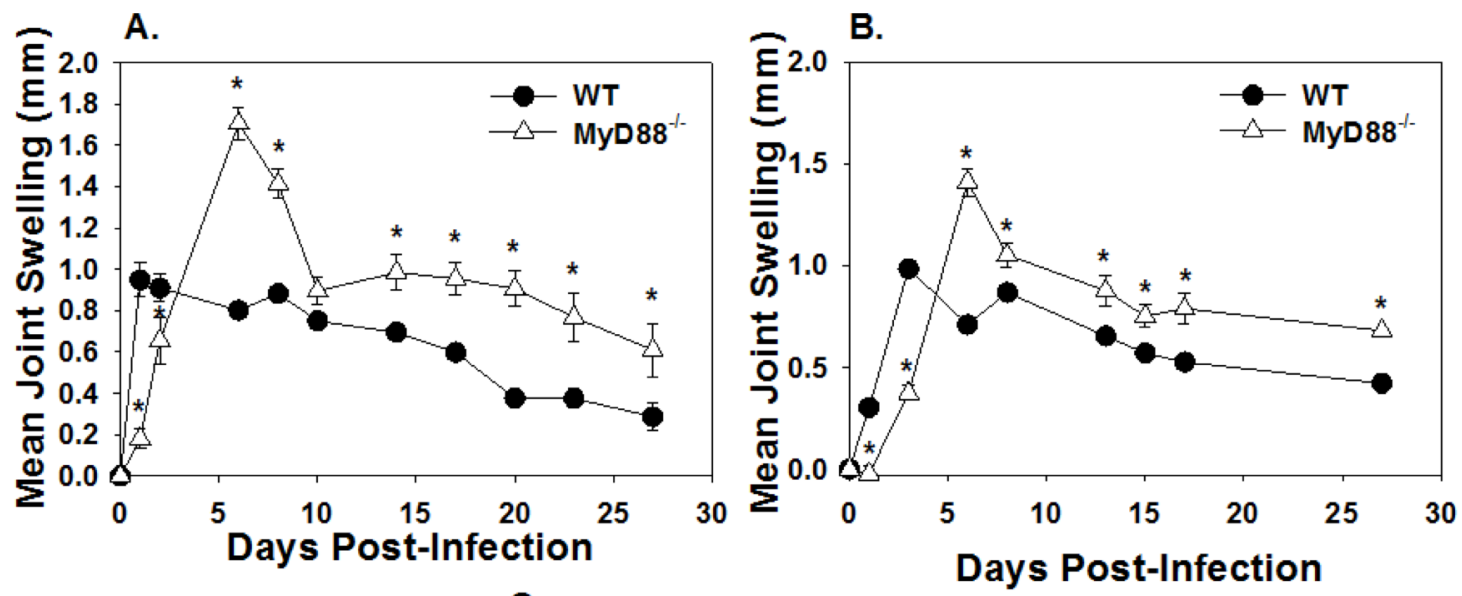

c.

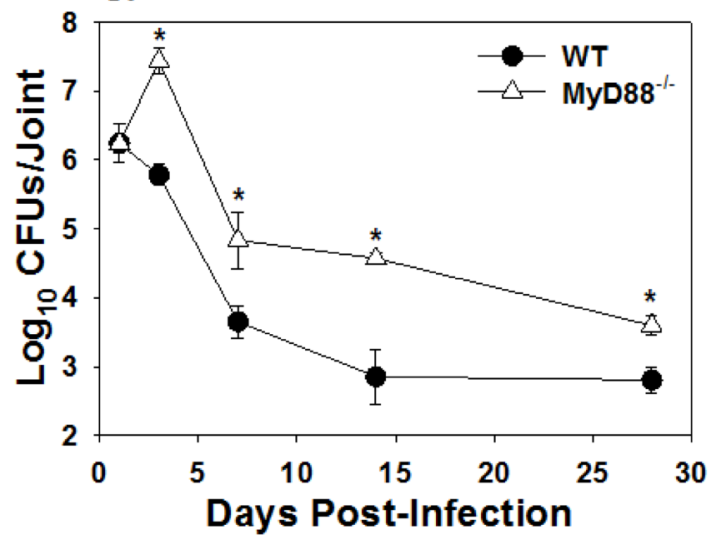

Figure 12. MyD88 ${ }^{-/-}$mice have delayed joint swelling but increased joint Brucella burden.

WT, C57BL/6, and MyD88 ${ }^{-/-}$mice were infected in each rear footpad with $1 \times 10^{6}$ (A) or with $1 \times 10^{5} \mathrm{~B}$. melitensis $16 \mathrm{M}$ (4-5 mice/group) (B) and swelling was recorded over time. At days $1,3,7,14$ and 28 , mice infected with $1 \times 10^{6} B$. melitensis were euthanized and bacterial loads were enumerated in the ankle joint (3-4 mice/group/timepoint) (C). P $<0.05$ as compared to WT mice. Error bars depict standard errors of the mean. CFU data in (C) is from one kinetic experiment. Swelling curves are representative of two independent experiments. 
conducted on wild-type C57BL/6 (WT) and MyD88 ${ }^{-1-}$ joints 3 days after infection with $1 \times 10^{6} \mathrm{CFUs}$ of B. melitensis $16 \mathrm{M}$ (Fig. 13A). Consistent with our swelling data,

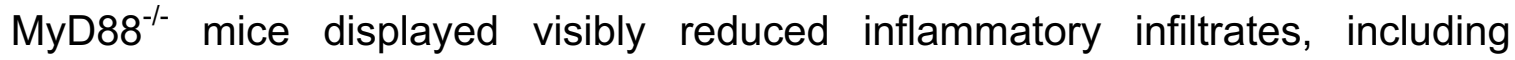
macrophages and neutrophils (Fig. 13A). Inflammation severity scores of MyD88${ }^{1-}$ joints at day 3 were significantly lower compared to infected WT mouse joints (Fig. 13B). Flow cytometry of infected joints also revealed reduced myeloid cell infiltration in MyD88 ${ }^{-/}$, relative to WT joints early in infection (data not shown). Despite reduced inflammation at early time points, MyD88 ${ }^{-/-}$mice developed similar pathology by day 7 and equaled or surpassed WT inflammation by day 14 and 28 post-infection (data not shown).

\section{MyD88 mediates inflammatory cytokine production in B. melitensis-infected mouse joints early in infection.}

The production of inflammatory cytokines can induce inflammation and arthritis $(197,198)$. To determine what inflammatory cytokines are MyD88dependent during B. melitensis-induced articular inflammation, cytokines were measured in ankle joint homogenates following footpad infection with $1 \times 10^{6} \mathrm{CFUs}$ of $B$. melitensis $16 \mathrm{M}$. At day 1 post-infection, the concentrations of the proinflammatory cytokines: CXCL2, CXCL1, IL-1 $\beta$, TNF- $\alpha$, CCL2, IL-6, CCL3 and IFNY were all significantly lower in MyD88 ${ }^{-/}$, compared to WT joint homogenates (Fig. 14A-H). However, by day 3 post-infection, TNF- $\alpha$ and IFN- $\gamma$ concentrations in MyD88 $^{-/-}$joints increased to WT levels (Fig. 14D\&H), while WT levels of CXCL1, CCL2 and IL-6 dropped and returned to similar levels as MyD88 ${ }^{-/-}$joints 


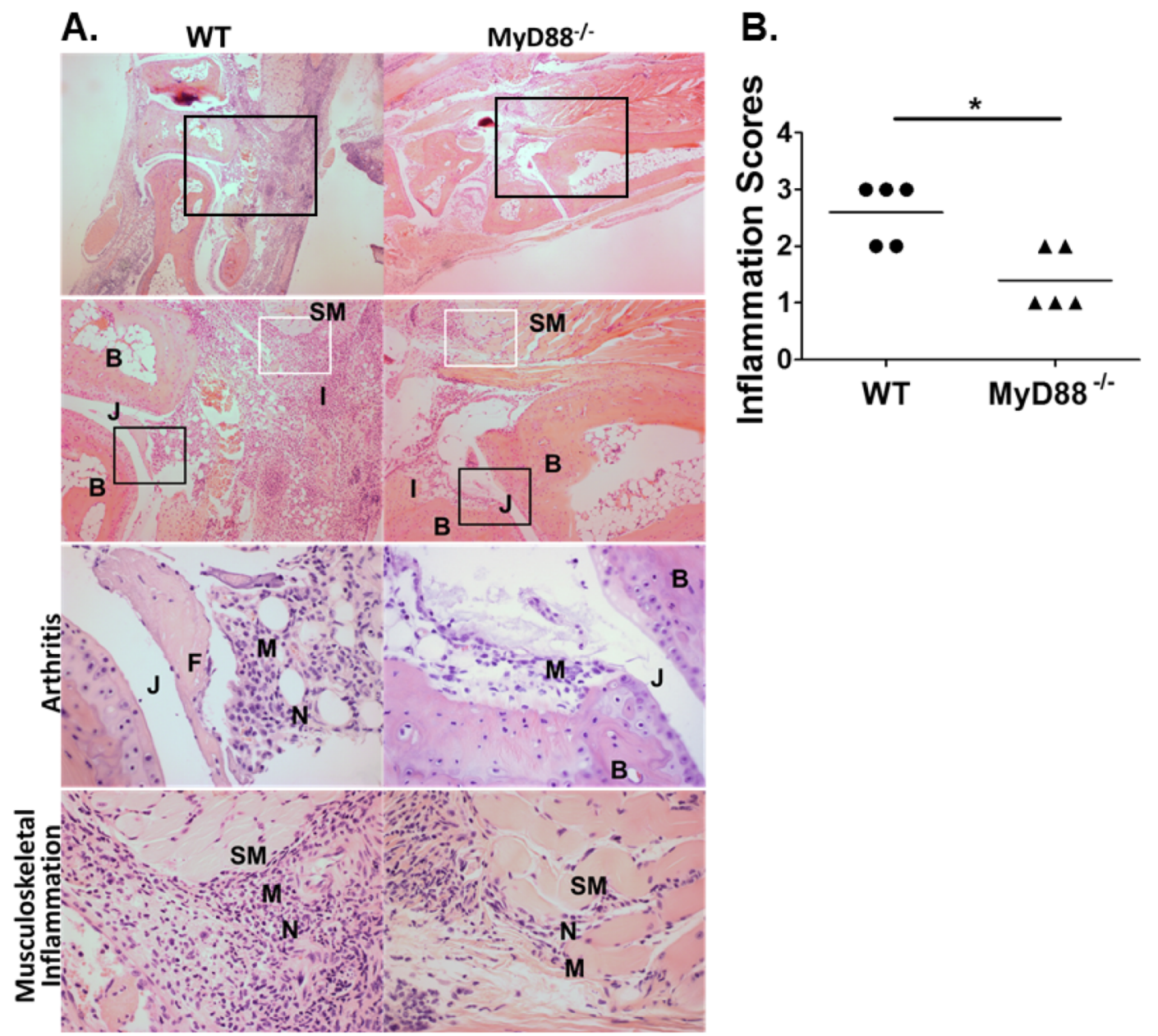

Figure 13. MyD88 mediates early arthritis and musculoskeletal inflammation.

WT, C57BL/6, and MyD88 ${ }^{-/-}$mice were infected in each rear footpad with $1 \times 10^{6}$ $B$. melitensis $16 \mathrm{M}$. Mice were euthanized 3 days post-infection and H\&E staining was conducted on mouse joints. Representative images of joint sections from WT and MyD88 ${ }^{-/-}$mice are depicted with amplified boxed regions displayed beneath each image (A). Magnification is as follows: row one $=40 \mathrm{x}$, row two $=100 x$, rows 3 and $4=400 x$. The black boxes indicate areas of arthritis or inflammation within and around the joint, and the white boxes indicate areas of musculoskeletal inflammation. Tissue architecture is indicated by: $\mathrm{B}=$ bone, $\mathrm{SM}=$ skeletal muscle and $\mathrm{J}=$ joint space. Areas of pathology are indicated by: $\mathrm{I}=$ inflammation, $\mathrm{M}=$ =macrophages, $\mathrm{N}=$ =neutrophils. Day $3 \mathrm{WT}$ and $\mathrm{MyD} 88^{-{ }^{-}}$ankle joint H\&E staining was scored for total inflammation (B). Histology scores are from one of two independent experiments (5 mice/group) from which sections were obtained. ${ }^{*} \mathrm{P}<0.05$ as compared to sections from WT mice. 

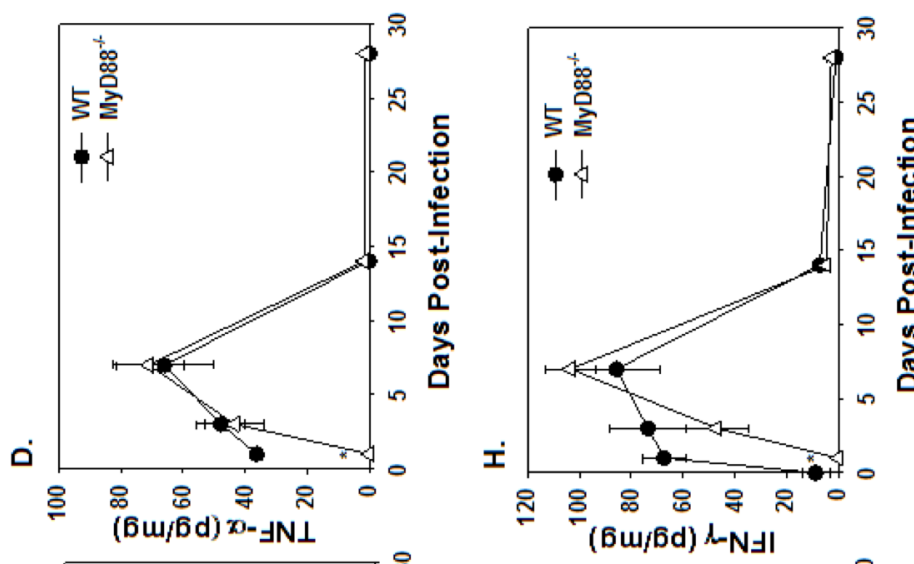

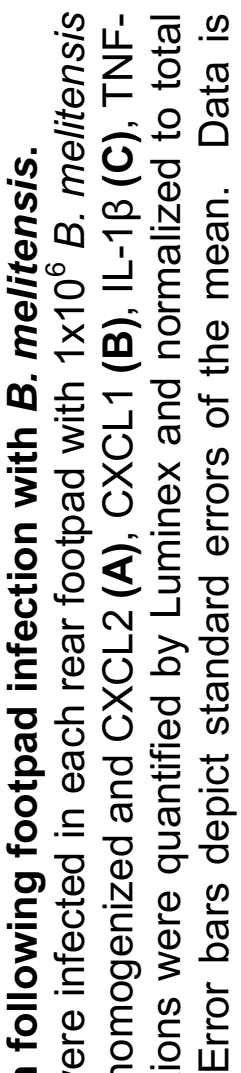

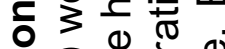
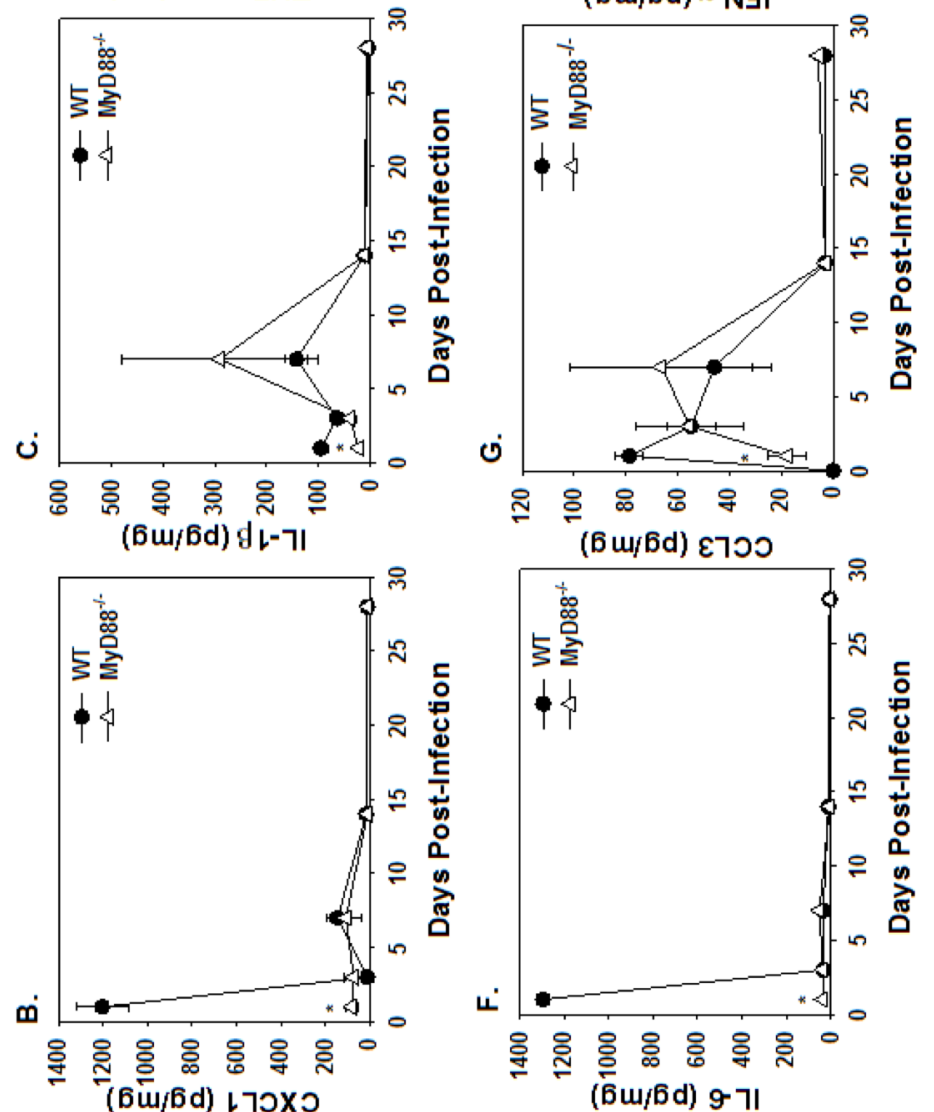

( $($ w/6d) عาวว

记

응 은

응 응

으음

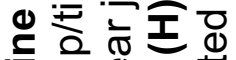

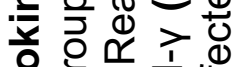

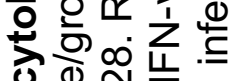

헝에

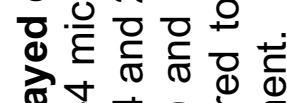

4.

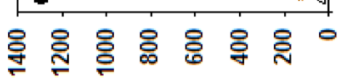

बे

ब이워

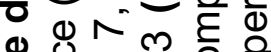

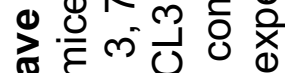

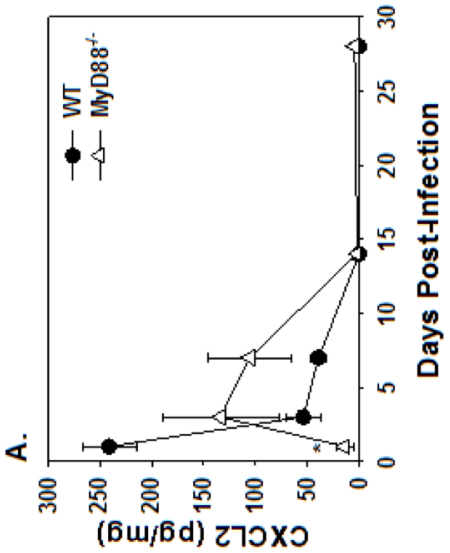

(5w/6d) 9 ר

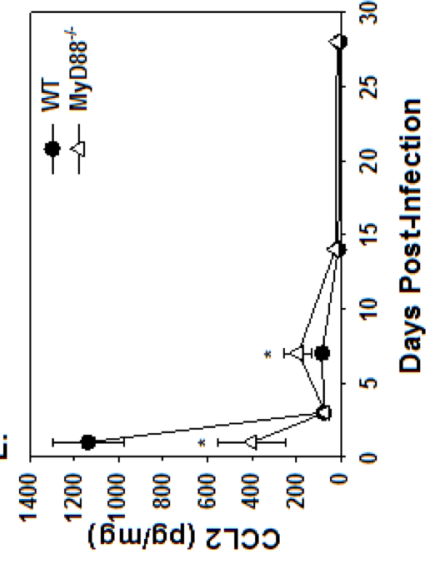

क⿻

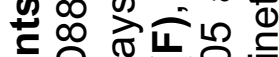

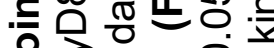

$\therefore \gtrless$

$\div$ 品口

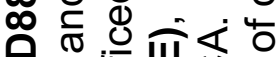

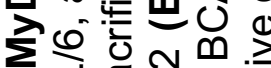

政

$\forall$ की

ఏ

站定

운 
(Fig. 14B\&E\&F). At day 3 post-infection IL-1 $\beta$, CCL3 and CXCL2 levels increased in MyD88 ${ }^{-/}$joints, while WT levels decreased (Fig. 14A,C,\&G). Eventually, all inflammatory cytokine concentrations became similar among groups by day 14 post-infection and at later time points.

\section{MyD88 aids in systemic Brucella clearance and T cell production of IFN-Y}

To determine if MyD88 is required for protection against systemic infection in this model, wild-type C57BL/6 (WT) and MyD88 ${ }^{-1-}$ mice were infected in the footpad with $1 \times 10^{6}$ CFUs of $B$. melitensis $16 \mathrm{M}$. Brucella spleen colonization in MyD88 $^{-1-}$ mice was similar to WT mice until day 14 ; however, at 28 days postinfection MyD88 ${ }^{-1-}$ mice had significantly increased splenic bacterial loads (Fig. 15A). To determine the role of MyD88 on systemic adaptive immune responses, $\mathrm{CD}^{+}$and $\mathrm{CD}^{+} \mathrm{T}$ cells from spleens of WT and MyD88 ${ }^{-/-}$mice were evaluated for IFN-y production. While the proportion of $\mathrm{CD}^{+}$and $\mathrm{CD}^{+} \mathrm{T}$ cells amongst splenocytes did not differ between WT and MyD88 ${ }^{-/-}$mice (data not shown), at days 7 and 14 post-infection, the percentage of IFN-ץ producing $C D 4^{+}$and $C D 8^{+}$ T cells was significantly lower in MyD88 ${ }^{-/}$mice compared to WT mice (Fig. 15B\&C). By day 28 post-infection, IFN-ץ production by T cells was similar between mouse strains (data not shown). 
A.

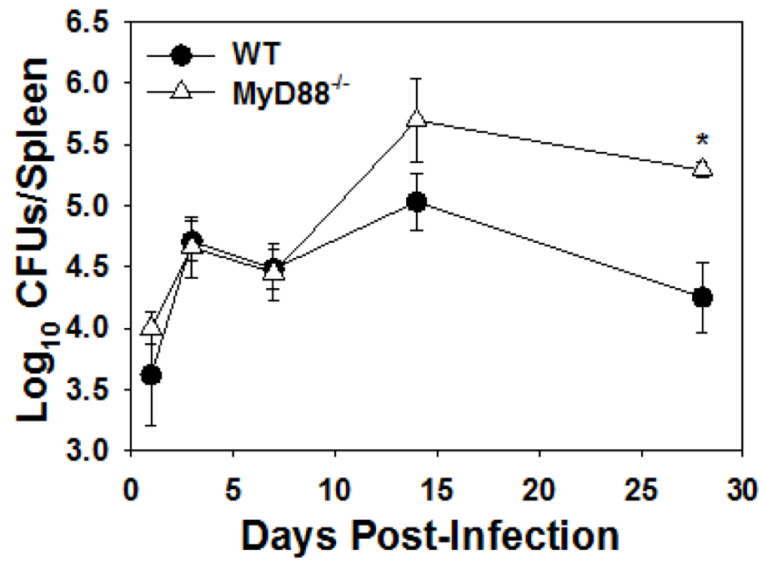

C.

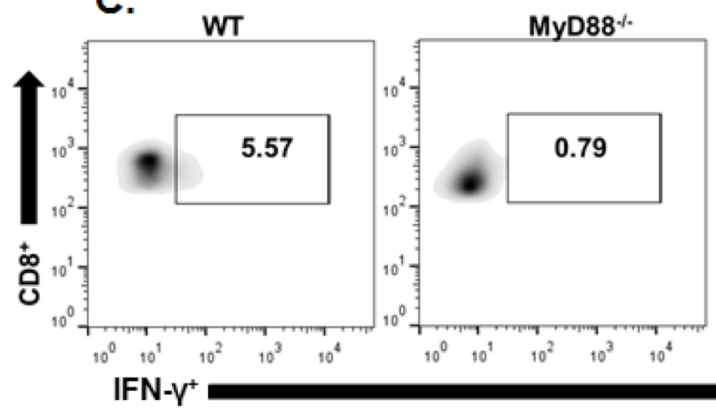

B.
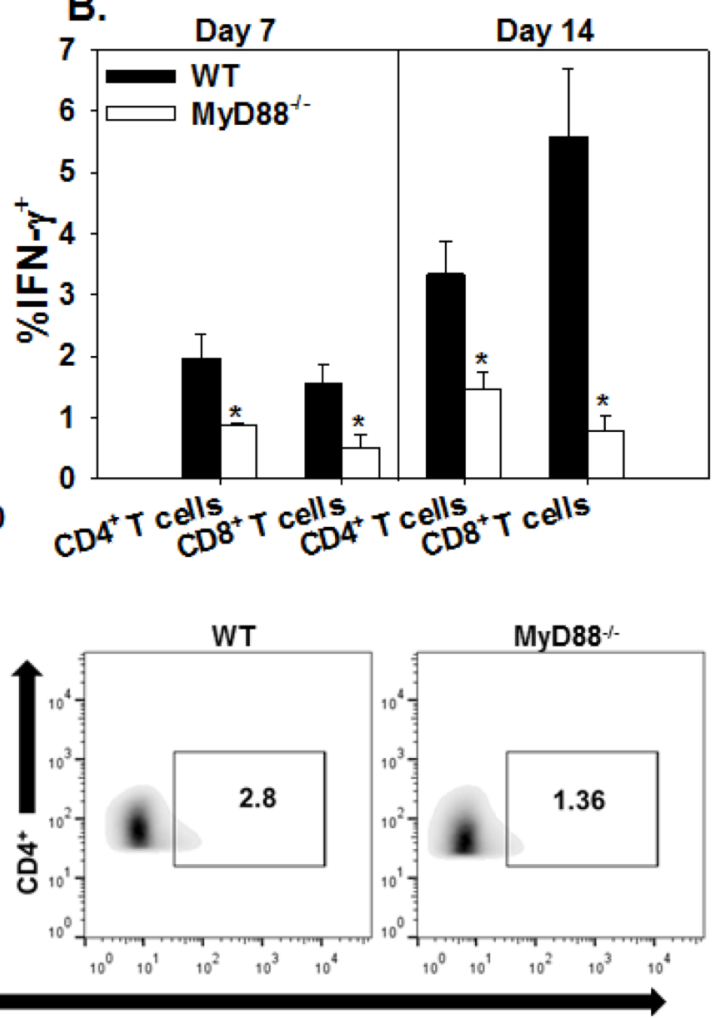

Figure 15. MyD88 aids in systemic Brucella clearance and T cell IFN-Y production.

WT, C57BL/6, and MyD88 ${ }^{-/-}$mice were infected in each rear footpad with $1 \times 10^{6}$ B. melitensis $16 \mathrm{M}$. At days $1,3,7,14$ and 28 , mice were euthanized and bacterial loads were enumerated in the spleen (3-4 mice/group/timepoint) (A). At days 7 and 14 flow cytometry was performed to determine IFN- $y$ production by $T$ cells from infected-mouse spleens (B). Representative plots showing IFNY production by gated $\mathrm{CD}^{+}$and $\mathrm{CD} 8^{+} \mathrm{T}$ cells from WT and MyD88 $8^{-/-}$mice 14 days post-infection (C). $\quad \mathrm{P}<0.05$ as compared to WT mice. Error bars depict standard errors of the mean. Data is representative of one kinetic experiment. 


\section{DISCUSSION}

With over 500,000 human cases reported each year, brucellosis is a globally important zoonotic disease (8). Arthritis and musculoskeletal inflammation are the most common focal symptoms of human brucellosis; $(7,8)$ however, the study of these manifestations has been hampered due to the absence of relevant mouse models. Intraperitoneal, pulmonary, or oral infection of wild-type, inbred, mouse stains with conventional doses of Brucella does not induce arthritis and musculoskeletal inflammation. Others have demonstrated that intra-articular inoculation of heat killed Brucella or Brucella lipoproteins into the knee induces osteoclastogenesis and recruitment of monocytes and neutrophils $(153,187)$. Footpad inoculation with Brucella spp. has been used to study Brucella dissemination and efficacy of vaccines (199-202), but inflammation in the joint and surrounding tissue was not described. Here we show that wild-type C57BL/6 mice inoculated via the footpad with $10^{3}-10^{6}$ CFUs of live Brucella develop arthritis and musculoskeletal inflammation. Similar to what has been described in human patients with articular brucellosis (63), and what we previously found in IFN- $\gamma^{-1-}$ mice, footpad infected mice also developed inflammation of musculoskeletal tissues surrounding the joint. To our knowledge this is the first description of a wild-type mouse strain developing arthritis and musculoskeletal inflammation when infected with a conventional dose of Brucella. We previously reported that IFN- $\gamma^{-1-}$ mice infected i.p. with B. melitensis developed exacerbated arthritis and joint colonization relative to IFN- $\gamma^{-/-}$mice infected i.p. with B. abortus (58). However, here we found that following footpad inoculation, B. melitensis, $B$. 
abortus, and B. suis-infected, mice all developed ankle swelling and exhibited similar joint bacterial loads and inflammation. Thus, the augmented arthritogenicity of $B$. melitensis observed in IFN- $\gamma^{-/-}$mice could be due to an enhanced ability to disseminate to the joint, rather than an enhanced ability to cause inflammation upon reaching the joint. Similar to what we previously reported in i.p.-infected IFN$\mathrm{Y}$-deficient mice (70), here we found that adaptive immune responses are not required for joint swelling in footpad inoculated mice. However, we did find that $B$ and/or T cells are requisite for resolution of joint infection and inflammation in this model. Thus, resolution of infection and inflammation in the footpad model could be due to adaptive immune cell production of IFN-Y.

Utilizing this footpad model of Brucella infection, we demonstrated MyD88 is important for eliciting early inflammatory responses within the first 3 days postinfection. Aspects of MyD88-dependent inflammation appear to be protective at later times, as MyD88--- mice had delayed resolution of inflammation. This may be due to increased bacterial loads as $\mathrm{MyD}^{-{ }^{--}}$mice had significantly higher joint bacterial burdens by day 3 post-infection compared to wild-type, C57BL/6 mice. We also demonstrated that MyD88 ${ }^{-/-}$mice had delayed expression of TNF- $\alpha$, IL$1 \beta$, CCL2, IFN- $\gamma$, and CXCR2 ligands. This is of interest as these cytokines were found at elevated levels in the synovial fluid from a human brucellosis patient with arthritic manifestations (66). MyD88 has previously been shown to be important for controlling systemic Brucella infection in mice (203). Likewise, MyD88 signaling has been reported to drive dendritic cell maturation and in turn T cell secretion of IFN- $\gamma$, which is protective against systemic Brucella infection $(141,204)$. In this 
model we demonstrated mice deficient in MyD88 have impaired $\mathrm{CD} 8^{+}$and $\mathrm{CD} 4^{+} \mathrm{T}$ cell production of IFN- $\gamma$ in their spleens. This suggests the footpad model of Brucella infection may be a useful tool for analyzing systemic control of inflammation and Brucella clearance. Another Brucella infection model demonstrated macrophage and dendritic cell production of IL-12 and TNF- $\alpha$ are MyD88 dependent during systemic infection (204). TLR2 and TLR6 can recognize and protect against systemic brucellosis, and there is varied evidence that TLR4 may also be involved $(108-111,203,205)$. TLR9 signaling can also increase the number of IFN- $\gamma^{+}$cells after i.p. infection (205). Furthermore, IL-1 and IL-18 signaling can utilize MyD88 for downstream neutrophil recruitment, NK and T cell activation, and production of inflammatory cytokines $(195,196)$. Thus, the early inflammation and joint cytokine production mediated by MyD88 are likely due to TLR activation and/or IL-1R/IL-18R relay which contribute to Brucella clearance at later times.

Although MyD88 promotes inflammation early after infection, another mechanism independent of MyD88 induces inflammation by day 7 . Joint levels of the inflammatory cytokines, TNF- $\alpha$, CXCL2, IL-1 $\beta$, CCL3 and IFN- $\gamma$, in mice lacking MyD88 elevated similarly to control animal levels by day 7, and swelling in

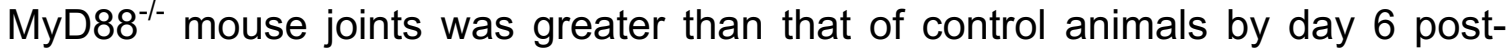
infection. This increase in joint inflammation in $M y D 88^{-/-}$mice may also be dependent on damage-associated molecular patterns induced by overwhelming bacterial burden, which could signal through MyD88 independent pathways (206). It is becoming increasingly clear that cytosolic signaling mechanisms may also 
induce inflammation upon Brucella infection. Although the MyD88-independent inflammatory pathway has yet to be determined in this model, one study demonstrated an important role for inflammasomes that require the apoptosisassociated speck-like protein containing a caspase-recruitment domain (ASC) in the host response to Brucella infection (117). Additionally, neutrophils infected with Brucella have been shown to induce TAK1 and SYK kinase-dependent oxidative bursts (207). Thus, the inflammasome, TAK1 and/or SYK kinase-dependent pathways could induce inflammation in mice lacking MyD88 in our model.

Although this is the first time MyD88 has been shown to be important for Brucella clearance in the joints, MyD88 is critical for joint pathogen clearance in Borrelia burgdorferi-induced arthritis. In contrast with Brucella infection, MyD88 does not appear to play a role in Lyme arthritis development $(208,209)$. In mice inoculated with E. coli or Streptococcus cell wall fragments, MyD88 is critical for the induction of arthritis and is dependent on TLR2 and TLR4, respectively (115, 116). Similarly, TLR4 antagonism can suppress autoimmune arthritis models, such as collagen-induced arthritis (210). It is clear that MyD88 is responding to Brucella infection of the joints, but the nature of the reacting cell types remain unknown. Numerous cell types in the joint, including macrophages, dendritic cells, synovial fibroblasts, chondrocytes, endothelial cells and fibroblast-like synoviocytes, are capable of responding to exogenous and endogenous TLR ligands and could be mediating inflammation in our model $(109,129,155,211-213)$.

In summary, we have described a footpad model of Brucella-infection that results in reproducible joint swelling, arthritis, and musculoskeletal inflammation 
following infection with B. melitensis, B. abortus or B. suis. One aspect of human infection that this model does not recapitulate is the dissemination of Brucella to the joint. However, local delivery of Brucella directly to the joint and surrounding tissue allows us to synchronize infection and inflammation. This synchronization allows us to investigate mechanisms of the host response that are specific to these tissues, whereas in a systemic infection, deficiencies in the host immune response could affect bacterial dissemination to the joint and thus make it difficult to determine if changes in joint inflammation are due to local immune responses, or due to a change in the dissemination of Brucella to the joint.

With this model we are also able to study Brucella-induced arthritis in the context of an intact IFN- $\gamma$ response. In IFN- $\gamma$-deficient mice, Brucella-induced focal inflammation does not resolve and the animals eventually succumb to infection after 4-8 weeks. In this footpad infection model, mice do not display systemic symptoms of disease (beyond colonization of the spleen and liver) and are able to slowly resolve inflammation, an effect that requires adaptive immunity. Thus, this novel model allows us to study both the initiation and resolution of focal inflammation due to Brucella infection. Here we utilized this footpad model and determined MyD88-dependent pathways are responsible for inducing early arthritis and musculoskeletal inflammation; however, MyD88-deficiency also leads to an increase in joint bacterial loads, which may delay resolution of articular inflammation. 


\title{
CHAPTER V
}

\section{Inflammasomes confer protection via IL-18 and pyroptosis, and are negatively regulated by IFN-y-dependent nitric oxide during Brucella infection}

\begin{abstract}
Brucellosis, caused by the intracellular bacterial pathogen Brucella, is a zoonotic disease for which arthritis is the most common focal complication in humans. Here we show caspase-1 and caspase-11 initiate Brucella-induced joint inflammation, but also restrict Brucella replication. In contrast, AIM2 and NLRP3 were dispensable for control of joint infection by Brucella. Both IL-1 and IL-18 promoted joint inflammation, however only IL-18 contributed to protection against Brucella infection of the joint. In vitro studies demonstrated caspase-1 and caspase-11 both induce pyroptosis which limited Brucella infection in macrophages. While early IFN-y production required caspase-1/11 and IL-18, caspase-1/11-dependent clearance of Brucella was not entirely IFN-y dependent. However, IFN-Y deficiency resulted in severe inflammation that was entirely inflammasome dependent and, in particular, reliant on NLRP3. IFN-y was vital for induction of the nitric oxide (NO) producing enzyme, iNOS, in infected joints, and NO inhibited caspase-1 activation in Brucella-infected macrophages in vitro. During Brucella infection in vivo, exacerbated joint inflammation was seen in iNOS deficient mice along with an increase in joint IL-1 $\beta$ compared to wild-type mice. Collectively these data demonstrate inflammasomes induce inflammation in an IL-18 and IL-1 dependent
\end{abstract}


manner, and inflammasome-dependent IL-18 and pyroptosis restrict Brucella infection. Moreover, IFN- $\gamma$ reduces inflammation by inhibiting excessive caspase1 activation through production of NO. Thus NO donors, in combination with antibiotics, may be an effective therapy for treating Brucella-induced inflammation.

\section{INTRODUCTION}

Identification of inflammatory responses contributing to articular brucellosis have been hindered by the lack of relevant mouse models. We have previously reported i.p. Brucella infection in IFN-y-deficient mice results in arthritis and musculoskeletal inflammation of ankle joints as early as 15 days post-infection. Arthritis in this systemic model was partially dependent on IL-1R and CXCR2, while adaptive immune cells were not required for inflammation $(70,165)$. It is currently unknown if IFN- $\gamma$ prevents arthritis by limiting Brucella dissemination to the joints, and/or if IFN- $\gamma$ acts locally in the joint to restrict infection and inflammation. Previously we demonstrated that mice inoculated with Brucella locally in footpads resulted in synchronized infection and joint inflammation in wild-type (WT) mice, allowing us to investigate mechanisms of the host response that are specific to the joint (160).

Inflammasomes are multi-protein structures that recognize threats in the host cell cytosol. Canonical inflammasomes are equipped with sensors that detect intracellular antigens and damage associated molecular patterns (DAMPs) (120). This initiates a cascade of protein oligomerization and recruitment resulting in inflammasome assembly. Upon activation, canonical inflammasomes recruit pro- 
caspase-1, a cysteine protease, and cleave it into its functional form, caspase-1 $(120,214)$. Caspase-1 can then activate pro-inflammatory cytokines as well as trigger a programmed inflammatory cell death known as pyroptosis $(120,215)$. Due to the inflammatory nature of inflammasomes they contribute to many models of inflammation including pathogen induced and sterile arthritis $(216,217)$.

Brucella infection in macrophages activates the canonical inflammasome cytosolic sensors: NLR family, pyrin domain containing 3 (NLRP3), and absent in melanoma 2 (AIM2). Studies have shown that the Brucella Type IV secretion system and mitochondrial damage can stimulate NLRP3, while Brucella DNA activates the AIM2 inflammasome pathway $(117,218,219)$. Following stimulation of the NLRP3 or AIM2 sensors and subsequent cleavage of caspase-1, active caspase-1 proteolyticly cleaves IL-1 $\beta$ and IL-18 into their functional/secreted forms. IL-1R and IL-18 are known to restrict Brucella burdens during chronic Brucella infection but may also lead to immunopathology, as they play a proinflammatory role in rheumatoid arthritis $(117,136,137,219)$. While NLRP3, AIM2 and caspases-1/11 are protective against chronic systemic Brucella infection in vivo (117), there are multiple studies indicating NLRP3 and/or AIM2 contribute to pathology in autoreactive arthritis models $(217,220,221)$. Thus, while inflammasomes contribute to restriction of systemic Brucella infection, they could also induce deleterious joint inflammation conferred by Brucella.

Unlike canonical inflammasomes, the non-canonical inflammasome, caspase-11, does not use additional cytosolic protein sensors to identify intracellular threats; rather, caspase-11 directly recognizes, and is activated by, 
cytosolic bacterial LPS. Like caspase-1, caspase-11 can also induce pyroptosis which can aid in the clearance of gram-negative intracellular pathogens (215). There is limited knowledge on Brucella's ability to activate caspase-11. In fact, one study showed the Brucella effector protein TcpB, can induce degradation of caspase-11 (122). However to our knowledge the role of caspase-11 in response to Brucella in vivo has not been directly examined. Here we describe a role for both caspase-1 and caspase-11 in Brucella induced arthritis and define the inflammasome effectors responsible for inflammation and control of infection. Additionally, we reveal IFN- $\gamma$ induced NO is able to suppress inflammasome activation.

\section{RESULTS}

\section{Inflammasomes have IL-1-independent effects on inflammation and control of infection during Brucella-induced arthritis}

Following intraperitoneal Brucella infection, IL-1R contributes to the development of Brucella induced arthritis in IFN-Y-deficient mice (165). Thus, to examine if inflammasome dependent IL-1 contributes to joint inflammation during local B. melitensis infection we infected caspase- $1 / 11^{-/-}$and IL-1 $\mathrm{R}^{-/-}$mice in the rear

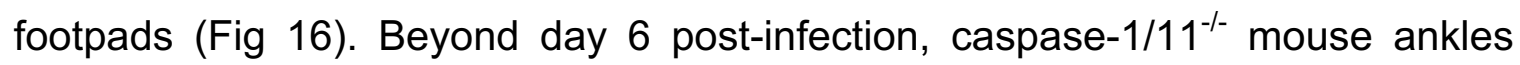
displayed variable but slightly increased swelling compared to WT animals, a trend which was also evident when a higher dose of Brucella was used for infection (Fig 

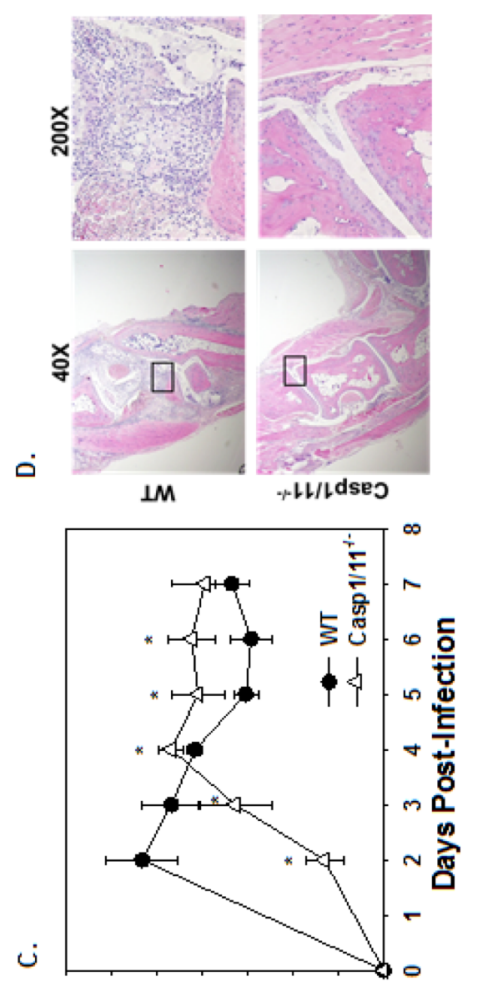

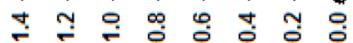
(uw) 6u!ा|әмs łu!̣or ueaw
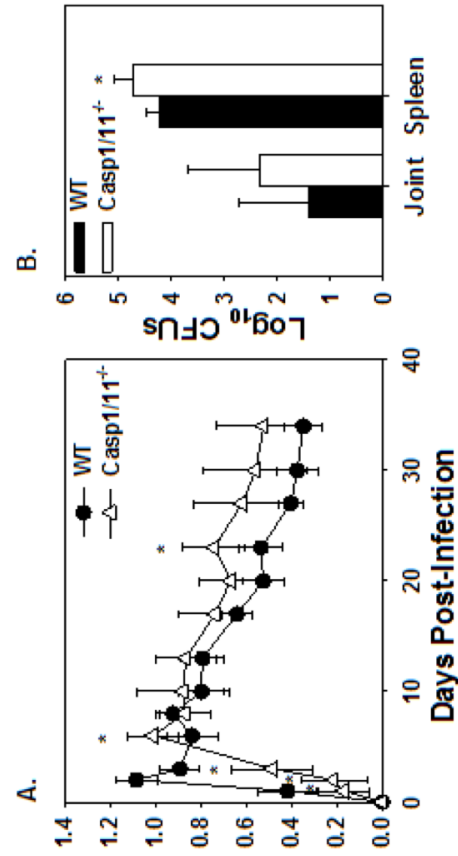

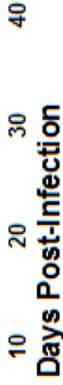

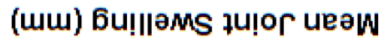

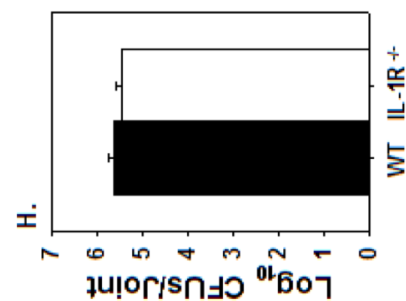

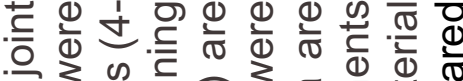
凶

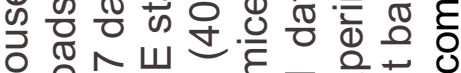

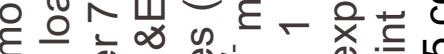

즈워

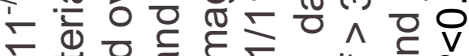

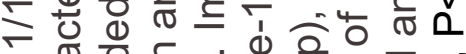

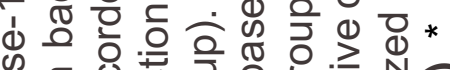
đิ

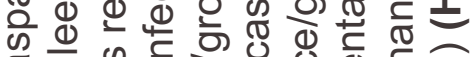

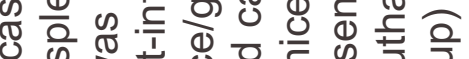
ऽ

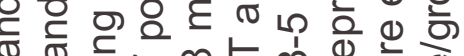

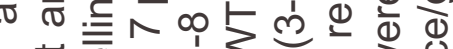
:

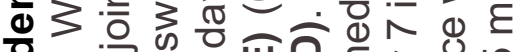

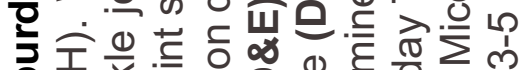

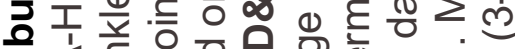
ब过完

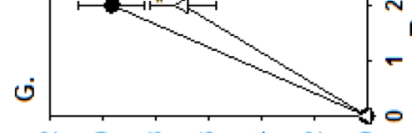

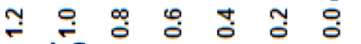

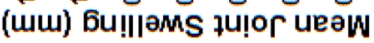

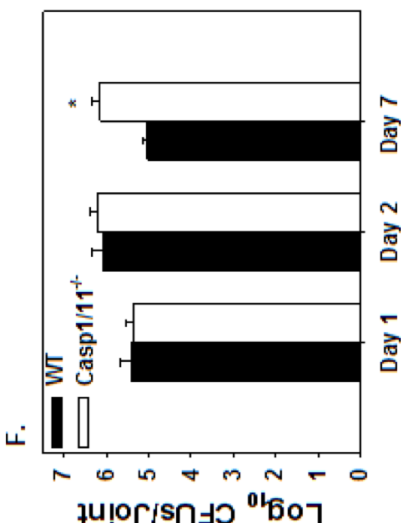

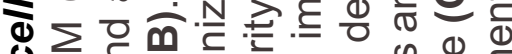
ง ต. .

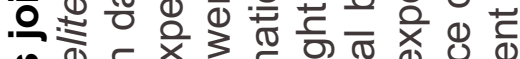

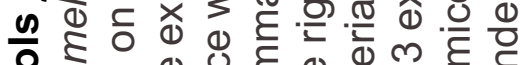

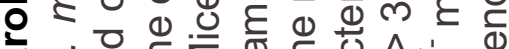
我

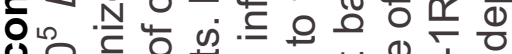

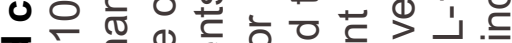

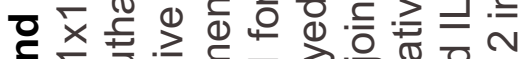

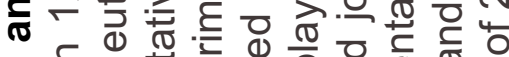

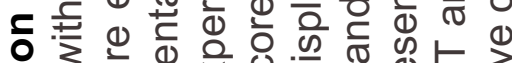

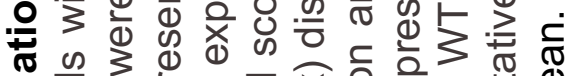

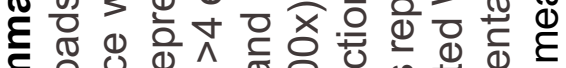
윤은 సั

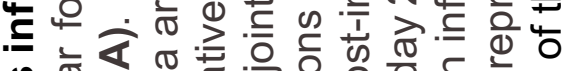
ต ঠั

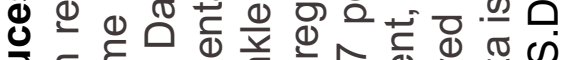
ग्ञ 응 윽웡 F.

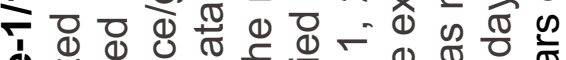

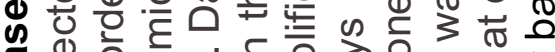
ชัญ

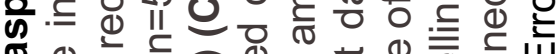
ง 닌 0 3 3 过

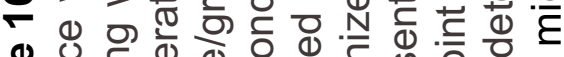
ญ

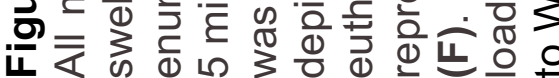


$16 \mathrm{~A}$ and Fig 17A). This long-term study revealed a minor role of caspase-1/11 in systemic clearance of Brucella from the spleen but only when a dose of $1 \times 10^{5} \mathrm{~B}$. melitensis was used for infection (Fig 16B and Fig 17B). Interestingly, caspase$1 / 11^{-/-}$mice exhibited delayed swelling with significantly reduced ankle diameters at days 2 and 3 compared to WT mice (Fig 16C). Consistent with our swelling data, H\&E staining revealed decreased inflammation in caspase- $1 / 11^{-/-}$joints at day 2 post-infection (Fig 16D-E). Additionally, caspase-1/11 $1^{-/-}$mice exhibited lower levels of inflammatory cytokines in their joints compared to WT mice (Fig 18A-F). In contrast to the phenotype observed at days 2 and 3 , caspase- $-1 / 11^{-/-}$mice had increased swelling by days 4-5 when compared to WT animals (Fig 16C). To explain this escalation of swelling in caspase- $1 / 11^{-/-}$mice we hypothesized inflammasomes may be important for mediating bacterial clearance in the joints. When joint bacterial loads were examined, caspase- $1 / 11^{-/-}$mice had similar CFUs compared to WT at days 1 and 2 post-infection; however, by day 7 caspase-1/11 I- animals had significantly increased Brucella loads (Fig 16F). To examine whether IL-1 was inducing this arthritis and musculoskeletal inflammation, swelling of WT and $\mathrm{IL}-1 \mathrm{R}^{-/-}$mouse ankles was recorded over 7 days post-Brucella infection. Interestingly, IL-1 $\mathrm{R}^{-/-}$mice only displayed slightly reduced swelling at day 2 , and Brucella loads at day 7 were similar among groups (Fig 16G\&H). This disparity in

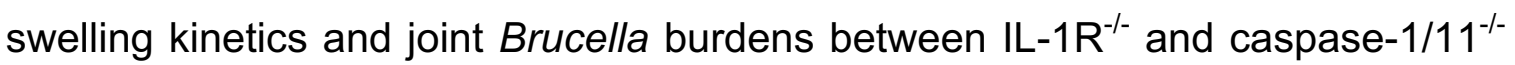
mice suggests inflammasomes have IL-1R independent effectors that contribute to Brucella-induced arthritis and control of infection. 
A.

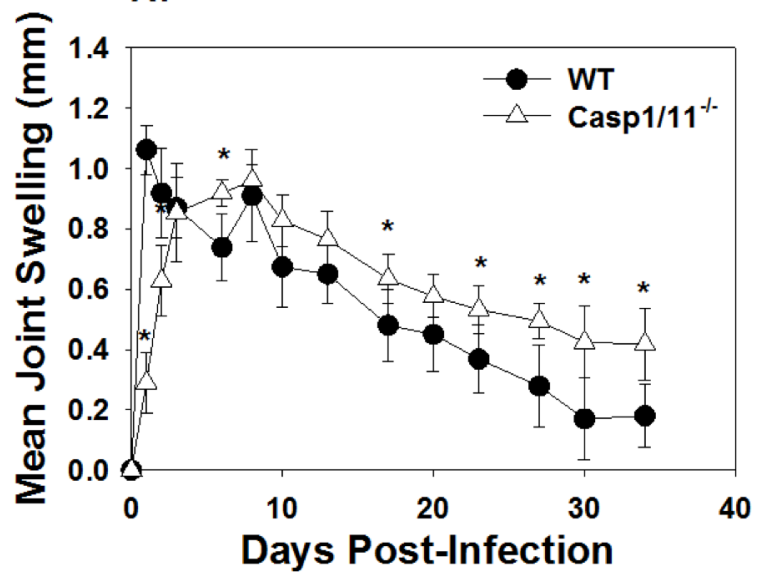

B.

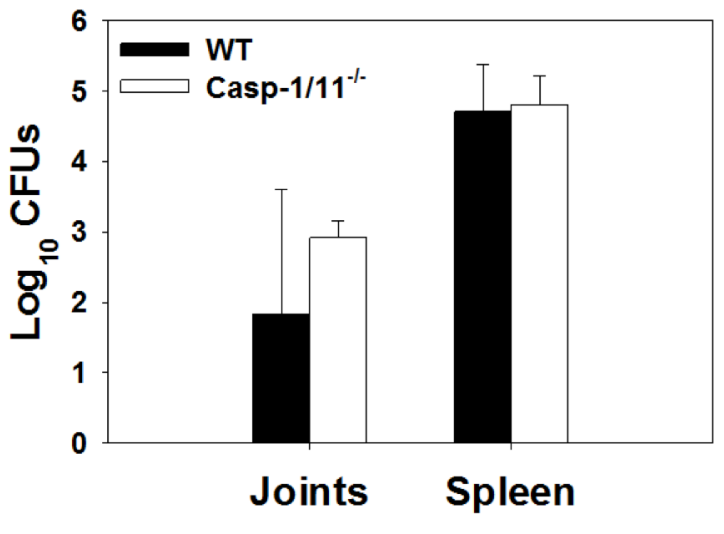

Figure 17. Caspase1/11 deficiency results in delayed resolution of joint swelling.

WT and caspase- $1 / 11^{-/-}$mice were infected in each rear footpad with $1 \times 10^{6} B$. melitensis $16 \mathrm{M}$ and swelling was monitored (A). Mice were euthanized on day 34 post-infection and spleen and joint Brucella burdens were enumerated (5 mice/group) (B). ${ }^{*}, \mathrm{P}<0.05$ compared to WT mice. Error bars depict S.D. of the mean. Data are representative of one experiment. 
A.

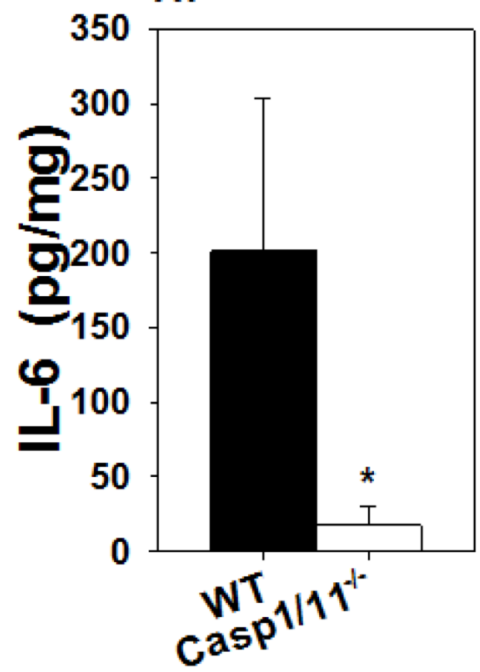

D.

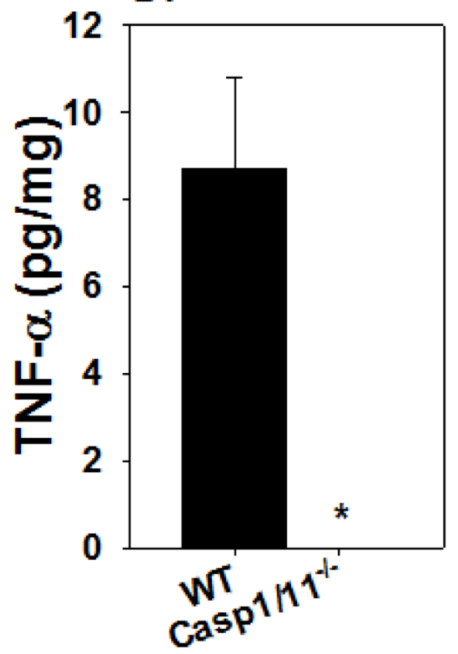

B.

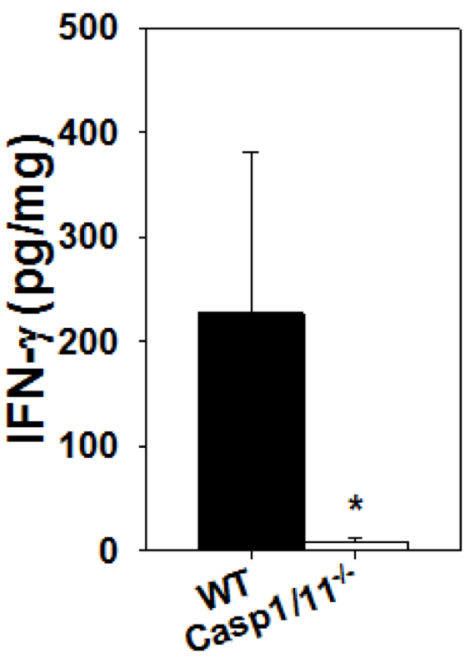

E.

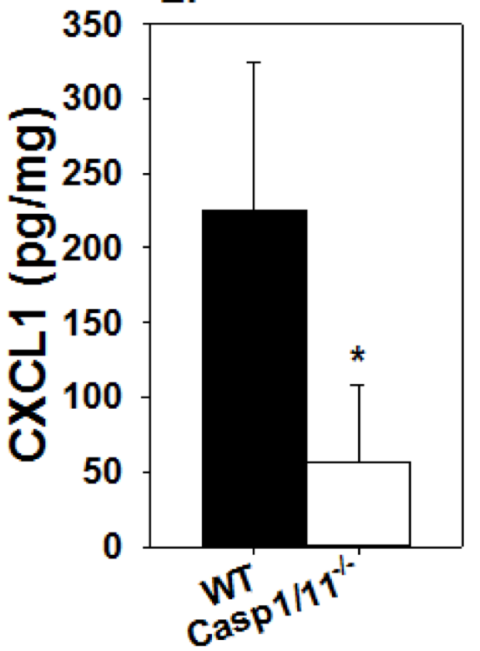

C.

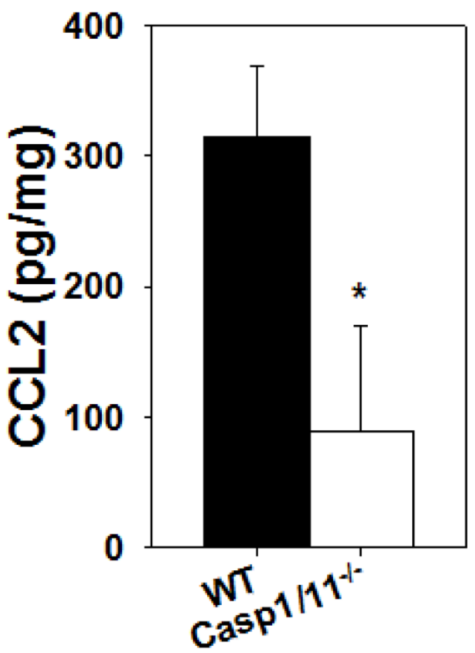

F.

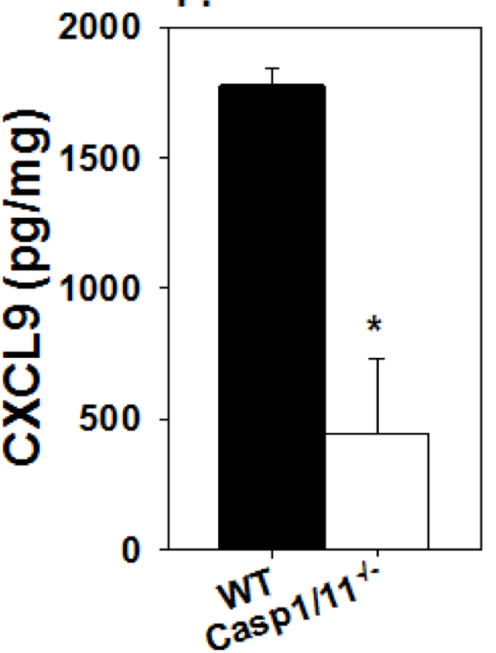

Figure 18. Inflammasomes mediate pro-inflammatory cytokine production in Brucella-infected joints.

WT and caspase- $1 / 11^{-/-}$mice were infected in each rear footpad with $1 \times 10^{5} \mathrm{~B}$. melitensis $16 \mathrm{M}$. Mice were euthanized at day 2 post-infection and levels of joint cytokines were determined. Data are representative of two independent experiments. ( $\mathrm{n}=3$ mice/group) (A-F). ${ }^{*}, \mathrm{P}<0.05$ compared to WT mice. Error bars depict the S.D. of the mean. 


\section{Caspase-11 contributes to Brucella-induced arthritis and restricts Brucella infection in the joint}

Many studies utilize caspase- $1 / 11^{-/-}$mice to study the role of caspase- 1 despite the fact that these animals also lack functional caspase-11. To determine if the non-canonical inflammasome, caspase-11, contributes to the initiation of inflammation and/or the clearance of Brucella from the joint, WT and caspase-11'

${ }^{1-}$ mice were infected in the rear footpads with $B$. melitensis. Indeed caspase- $11^{-1-}$ mice displayed reduced swelling at day 2 post-infection, while day 2 joint bacterial loads remained similar to WT (Fig 19A and data not shown). The reduction of joint swelling in caspase- $11^{-1-}$ mice relative to WT animals was not to the same magnitude as that seen in caspase- $1 / 11^{-/-}$mice, suggesting a partial role of caspase-11 in inflammasome dependent induction of swelling. Histopathology analysis at day 2 post-infection confirmed caspase-11's inflammatory role, as caspase-11/- joints had reduced inflammation compared to WT (Fig 19B\&C). Additionally, IL-6 and TNF- $\alpha$ levels were significantly decreased in caspase- $11^{-/-}$ joints at day 2 post-infection (Fig 20A-F). Caspase- $11^{-/-}$mice also displayed increased joint swelling by day 5 post-infection (Fig 19A). To examine the effects of caspase-11 on bacterial clearance, we measured joint bacterial burdens on day

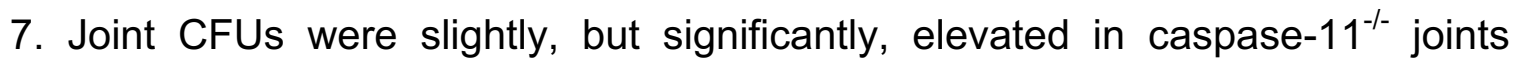
compared to WT mice (Fig 19D). Similar to our swelling data, the difference in CFUs between caspase- $11^{-/-}$and WT mice was not to the same magnitude as the 

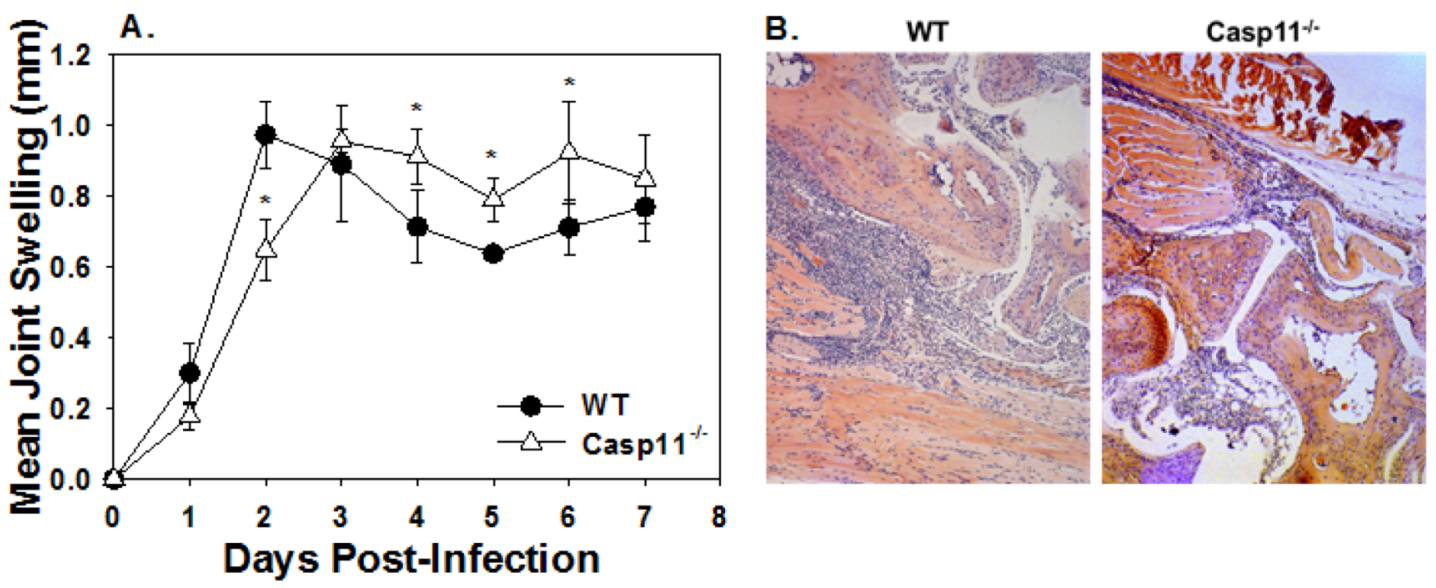

C.

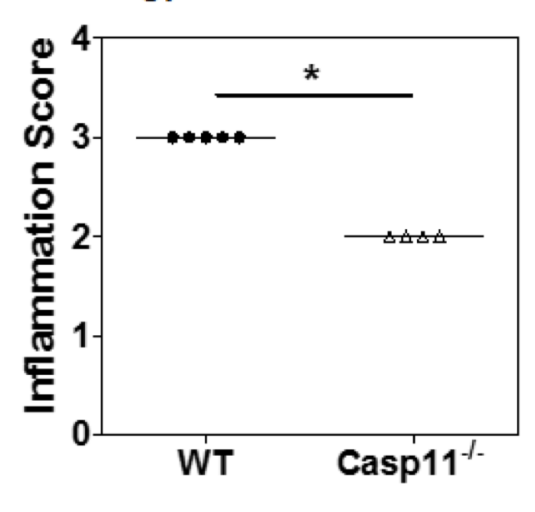

D.

E.
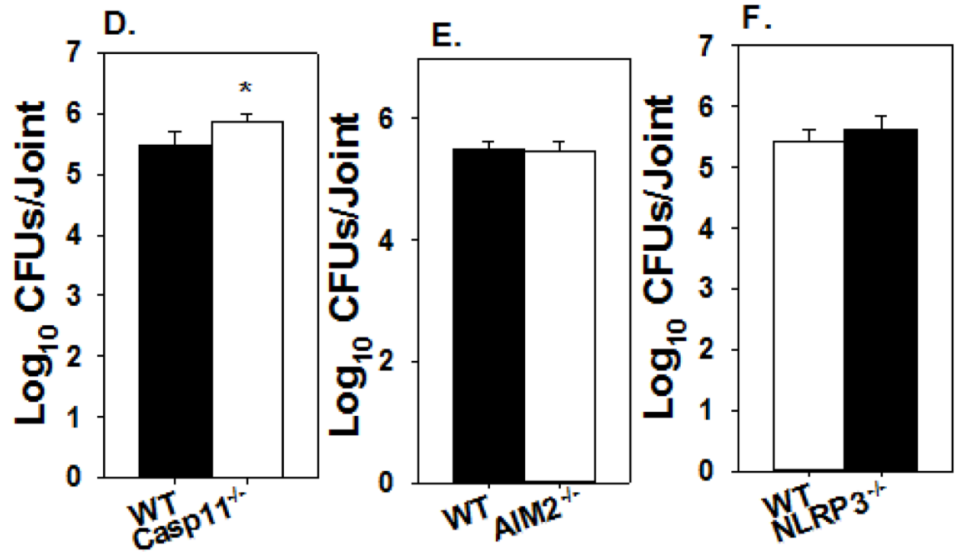

Figure 19. Caspase-11 is inflammatory and limits Brucella infection in the joints.

All mice were infected in each rear footpad with $1 \times 10^{5} \mathrm{~B}$. melitensis $16 \mathrm{M}$. Joint swelling was measured over time in WT and caspase- $11^{-/}$mice. Data representative of 3 independent experiments (4-5 mice/group) (A). Mice were euthanized at day 2 post-infection and H\&E staining was conducted on mouse ankles. Representative images of joint sections from WT and Caspase- $11^{-1-}$ mice are depicted (100x) (B). Severity of pathology was then scored. Histology scores are from one of two independent experiments (4-5 mice/group) from which sections were obtained (C). Mice were euthanized on day 7 post-infection and joint Brucella loads were enumerated. Data are representative of 2 independent experiments (4-5 mice/group) (D). $\mathrm{NLRP}^{-1-}$ and $\mathrm{AIM} 2^{-/-}$mice were evaluated for joint Brucella burdens at day 7 post-infection. Data are representative of 2 independent experiments (4-5 mice group) (E\&F). ${ }^{*}, P<0.05$ compared to WT mice. Error bars depict S.D. of the mean. 
A.

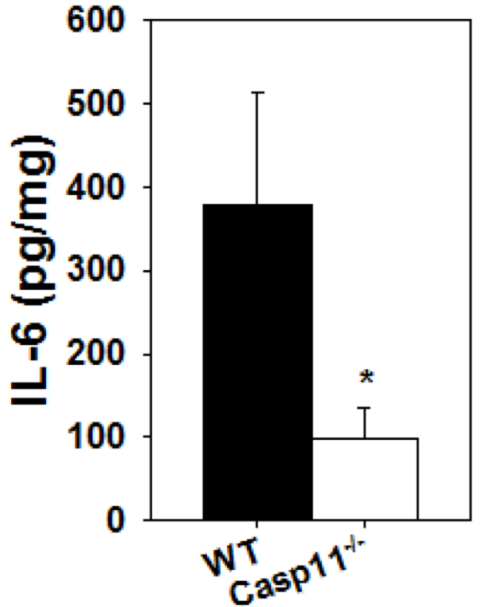

D.

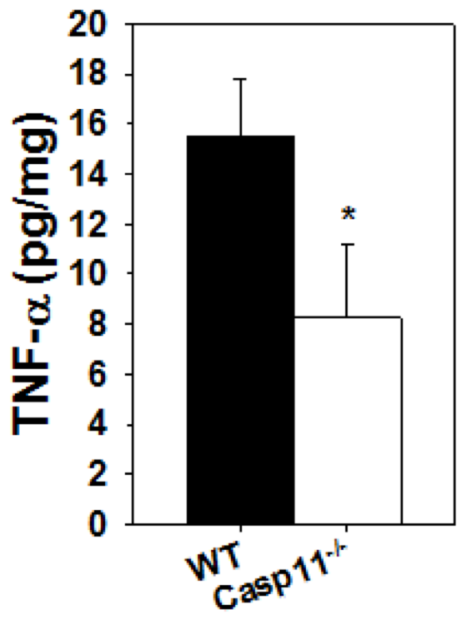

B.

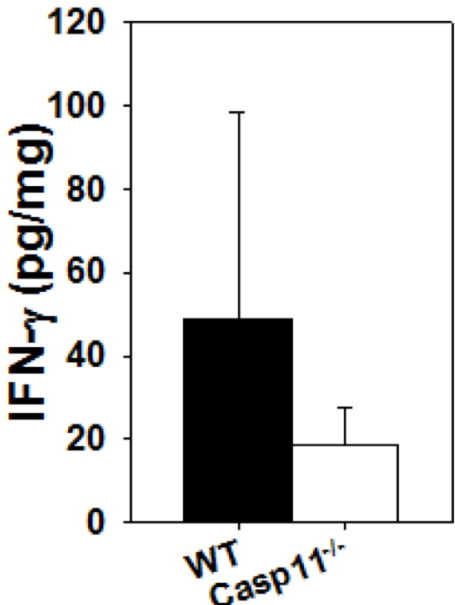

E.

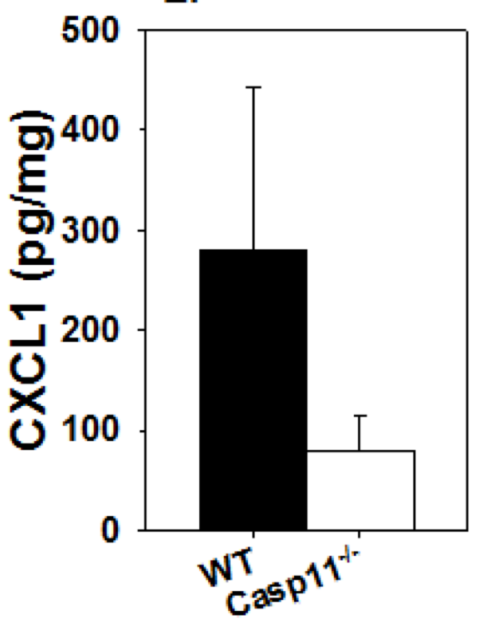

C.

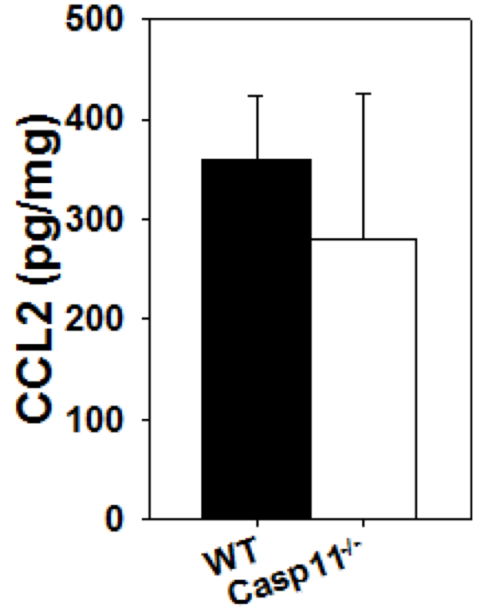

F.

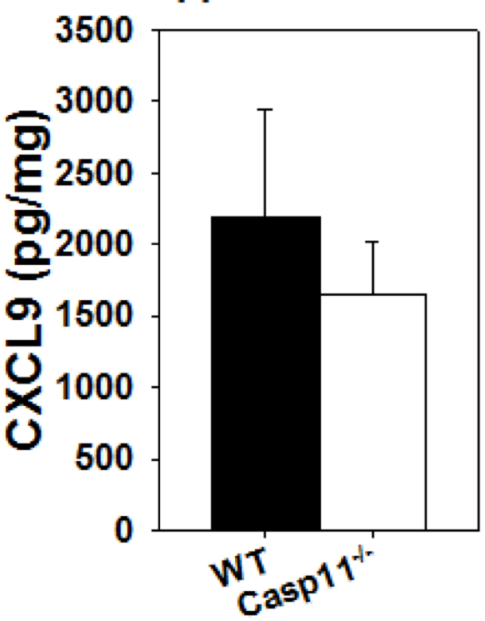

Figure 20. Caspase-11 contributes to inflammasome dependent proinflammatory cytokine production in Brucella-infected joints.

WT and caspase- $11^{-1-}$ mice were infected in each rear footpad with $1 \times 10^{5} \mathrm{~B}$. melitensis $16 \mathrm{M}$. Mice were euthanized at day 2 post-infection and levels of joint cytokines were determined. Data are representative of one experiments. ( $n=3$ mice/group) (A-F). * $P<0.05$ compared to WT mice. Error bars depict the S.D. of the mean. 
difference between caspase $-1 / 11^{-/-}$and WT mice. To assay what canonical inflammasome could be involved in caspase-1 dependent control of Brucella infection, $\mathrm{NLRP}^{-/-}$and $\mathrm{AIM}^{-/-}$mice were infected and evaluated for Brucella burdens at day 7 post-infection. Interestingly, $\mathrm{NLRP}^{-/-}$nor AIM2 ${ }^{-1-}$ mice displayed impaired ability to control joint Brucella burdens (Fig 19E\&F).

\section{Caspase1/11-dependent IL-18 mediates joint swelling and Brucella clearance from the joint}

In addition to IL-1 $\beta$, inflammasomes also activate IL-18. To investigate if IL18 is an inflammasome-dependent effector of Brucella-induced arthritis, IL-18 was neutralized in WT and caspase-1/11 $1^{-/}$mice and the impact on arthritis assessed. Joint swelling 2 days post-infection was reduced in anti-IL-18-treated mice compared to WT animals, but IL-18 neutralization did not have as great of an effect

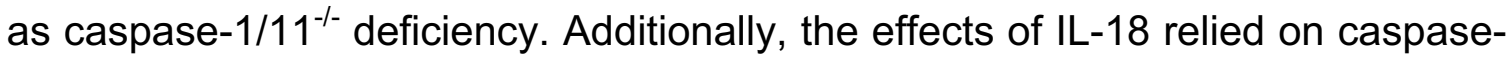
$1 / 11$, as swelling in caspase $-1 / 11^{-/-}$mice was similar to caspase- $-1 / 11^{-/}$-anti-IL-18 treated animals (Fig 21A). Bacterial loads at day 2 post-infection were similar

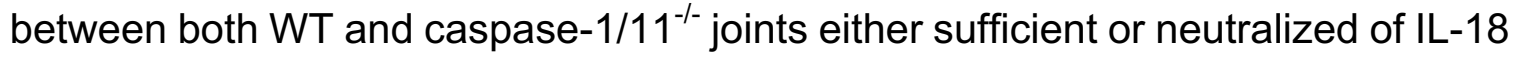
(Fig 21B). To examine if IL-18 contributes to swelling or joint bacterial restriction beyond the initial initiation of inflammation, swelling in $\mathrm{IL}-18^{-/-}$mice was monitored over the course of 7 days. IL-18 ${ }^{-/} / / \mathrm{L}-1 \mathrm{R}^{-/-}$mice were also included in our study to further examine the role of IL-1R in our model. IL-18 ${ }^{-/-}$mice only had reduced joint swelling at day 2 post-infection while $\mathrm{IL}-18^{-/} / \mathrm{IL}-1 \mathrm{R}^{-/-}$mice had significantly reduced 
A.

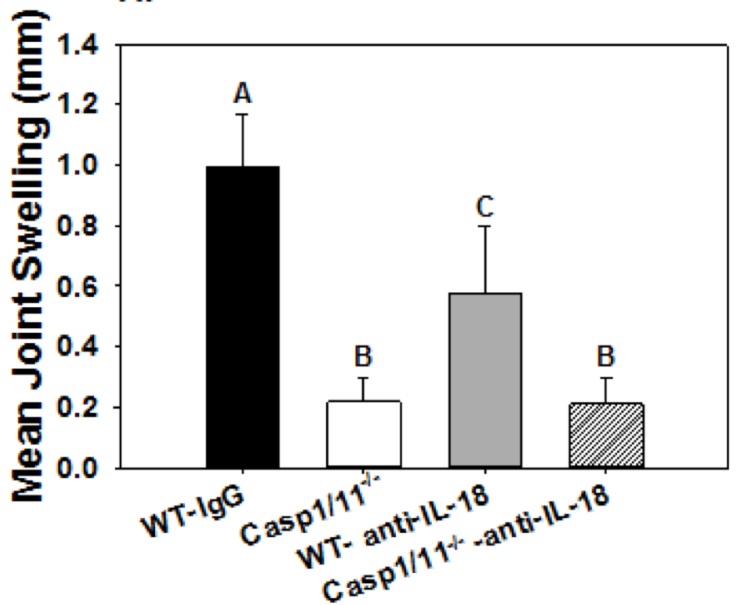

C.

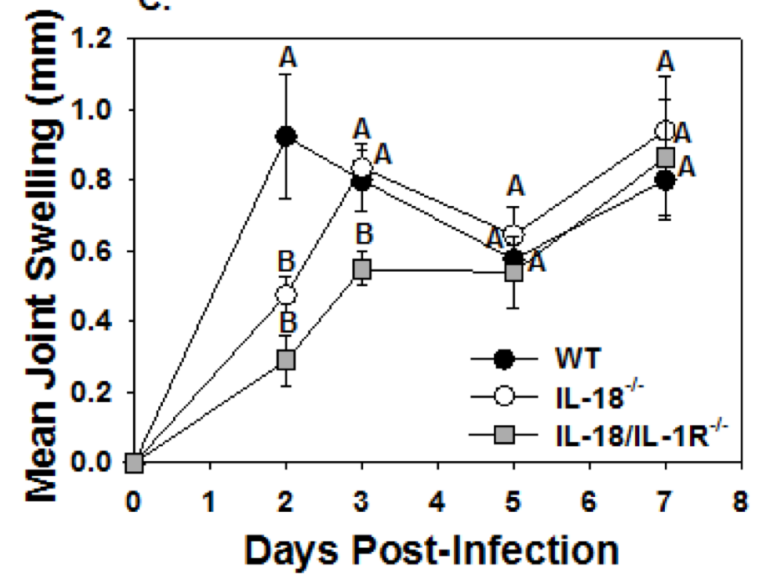

B.

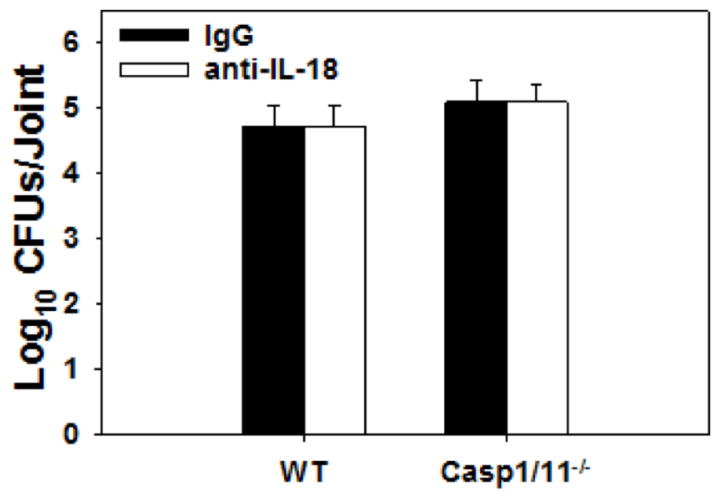

D.

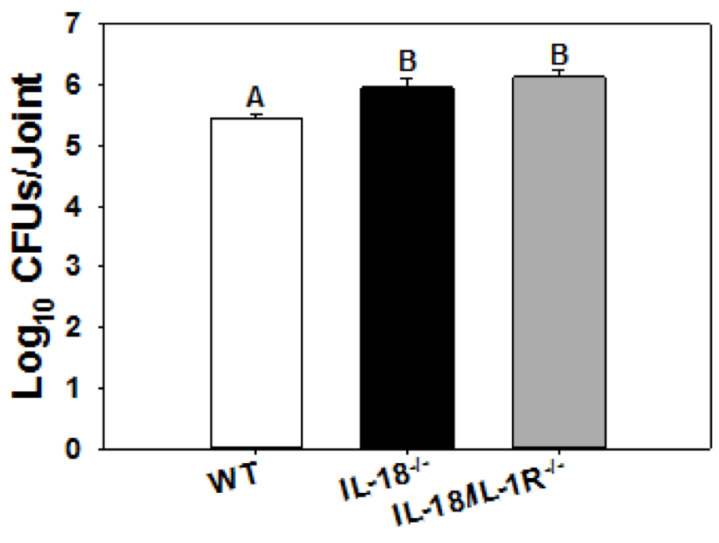

Figure 21. IL-18 contributes to inflammasome dependent joint inflammation and Brucella clearance.

WT or caspase- $1 / 11^{-/-}$mice were treated with an IgG isotype control or anti-IL18 then infected in each rear footpad with $1 \times 10^{5}$ B. melitensis $16 \mathrm{M}$ (A-B). At day 2 post-infection ankle swelling was recorded (A) and joint CFUs were enumerated. Data are combined from 2 separate experiments 8-10 mice/group total (B). WT, IL-18 $8^{-/-}$and IL-1R/IL-18 $8^{-/-}$mice were infected in the rear footpad with $1 \times 10^{5} \mathrm{~B}$. melitensis $16 \mathrm{M}$ and swelling was recorded over time (C). Mice were euthanized on day 7 post-infection and joint bacterial burdens determined. Data are representative of 2 independent experiments (4-5 mice/group) (D). Means with the same letter are not statistically different from each other as determined by ANOVA followed by Tukey's test. Error bars depict S.D. of the mean. 
ankle widths at day 2 and 3 when compared to WT mice (Fig 21C). By day 7 postinfection, IL-18 deficiency resulted in increased joint Brucella burdens, however IL$1 \mathrm{R}$ deficiency in IL-18 ${ }^{-/}$mice had no additive effect on control of joint infection (Fig 21D). These data indicate IL-18 and IL-1R are involved in the early induction of swelling during Brucella-induced arthritis, but IL-18 alone is required for restriction of Brucella infection in the joint. Relative to WT mice however, caspase-1/11 deficiency resulted in increased bacterial burdens and had a greater effect on joint swelling than IL-18/IL-1R deficiency. This suggests IL-18 and IL-1 are not the sole effectors of caspase-1/11.

\section{Eicosanoids and perforin-mediated cytotoxicity are dispensable for control of Brucella infection in the joint}

Although inflammasome activation of $\mathrm{IL}-1 \beta$ and $\mathrm{IL}-18$ are the best characterized modes of caspase-1/11-dependent antimicrobial activity, inflammasomes can protect against infection via other means, including inducing eicosanoid storms (222). Thus, we treated Brucella infected mice with the eicosanoid inhibitor, indomethacin. Indomethacin treatment reduced joint swelling at day 2 post-infection, but no significant difference in joint Brucella loads was observed at day 7 post-infection relative to WT mice (Fig 22A\&B). In fact, there was a trend of reduced Brucella burdens in indomethacin treated mice indicating eicosanoids are not bactericidal but actually may aid in Brucella colonization of the joint (Fig 22B). Additionally, IL-18 has recently been shown to activate perforinmediated cytotoxicity as a mode of intracellular bacterial clearance (134). Therefore, the role of cytotoxicity was evaluated using perforin ${ }^{-/}\left(\right.$perf $\left.^{-/}\right)$mice, 
A.

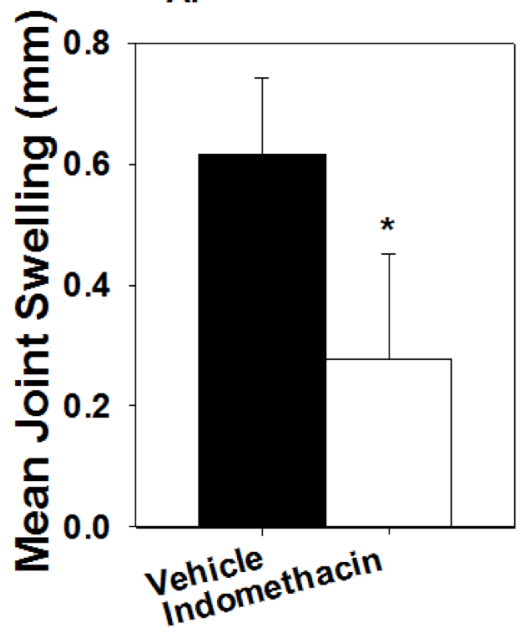

B.

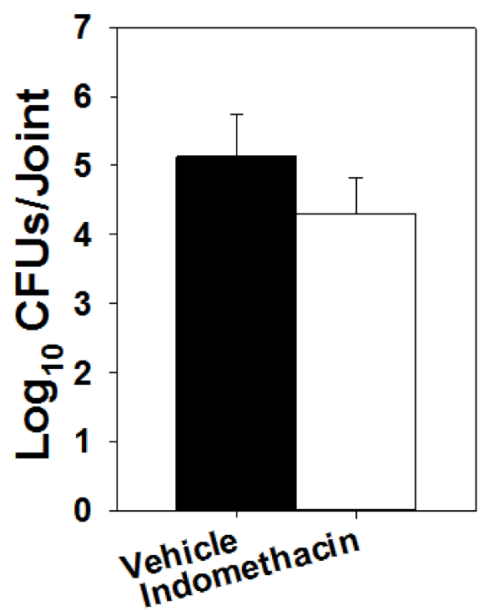

C.

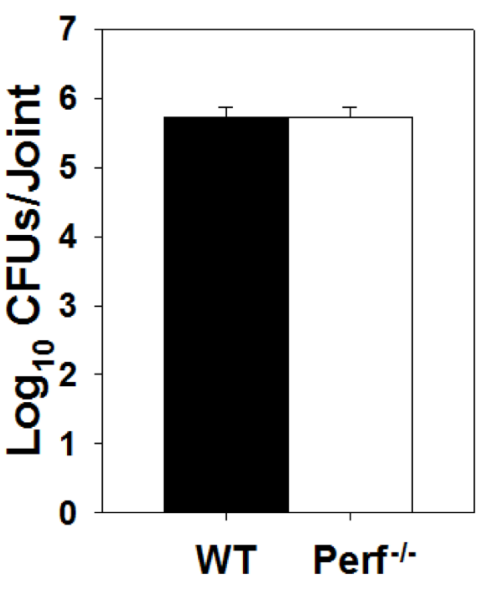

Figure 22. Eicosanoids and perforin are dispensable for control of Brucella infection in the joint.

Mice were either treated with indomethacin or a vehicle and were infected in each rear footpad with $1 \times 10^{5} \mathrm{~B}$. melitensis $16 \mathrm{M}$. At day 2 post-infection ankle swelling was measured (A). Mice were euthanized at day 7 post-infection and joint CFUs were enumerated (5 mice/group) (B). .,$P<0.05$ compared to vehicletreated mice. Data are representative of 2 independent experiments. WT and perf $^{-/}$mice were infected in each rear footpad with $1 \times 10^{6}$ B. melitensis $16 \mathrm{M}$. Mice were euthanized at day 6 post-infection and joint Brucella burdens were evaluated (5 mice/group) (C). Data are representative of one experiment. Error bars depict S.D. of the mean. 
but no notable changes in joint Brucella loads was observed (Fig 22C).

\section{Caspase-1 and caspase-11 induce cell death in response to Brucella and restrict infection in macrophages.}

Pyroptosis and subsequent efferocytosis of dead host cells containing bacteria can be an important antimicrobial mechanism of inflammasomes (215). To demonstrate if pyroptosis occurs during Brucella infection, BMDMs were infected with B. melitensis and evaluated for cell death at 24 and 48 hours postinfection. Brucella induced robust cell death in WT macrophages, while cell death was reduced in caspase- $11^{-/-}$macrophages and abolished in caspase- $1 / 11^{-/-}$cells at both 24 and 48 hours post-infection, indicating both caspase- 1 and caspase-11 contribute to Brucella-induced macrophage death. Interestingly $\mathrm{NLRP}^{-{ }^{--}}$and $\mathrm{AIM}^{-/-}$mice had similar amounts of cell death as WT macrophages, suggesting NLRP3 or AIM2 alone are not required to induce pyroptosis (Fig 23A\&B). We also observed elevated Brucella levels in wells with caspase $-1 / 11^{-/-}$and caspase- $11^{-/-}$ BMDMs at 24 and 48 hours post infection (Fig 23C\&D). Similarly, joint cells from footpad infected caspase-1/11 ${ }^{-/-}$mice displayed reduced Terminal Deoxynucleotidyl Transferase dUTP Nick-End Labeling (TUNEL), which identifies DNA fragmentation during late stage apoptosis or pyroptosis, compared to WT mice (223) (Fig 24A-B). To determine if caspase-11 could be directly activated by Brucella LPS, we treated peritoneal macrophages with purified Brucella abortus S19 LPS, which has a similar structure to $B$. melitensis LPS (224). We found that Brucella LPS was able to induce caspase-11-dependent cell death, 
A.

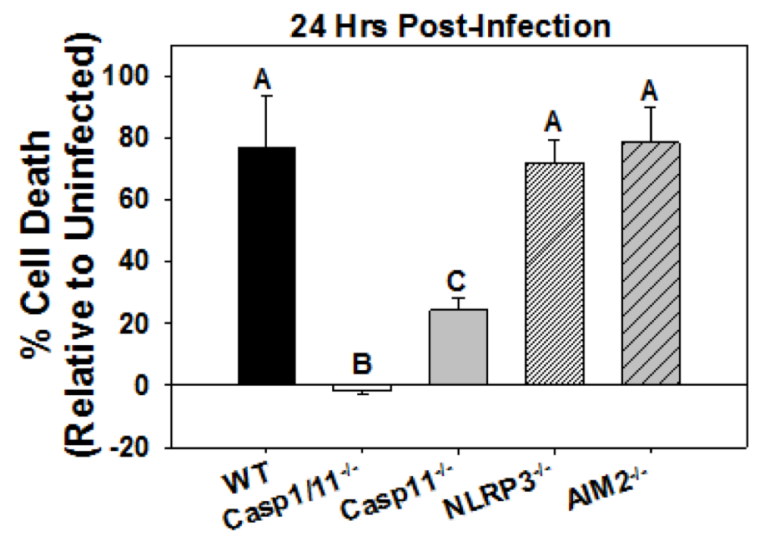

c.

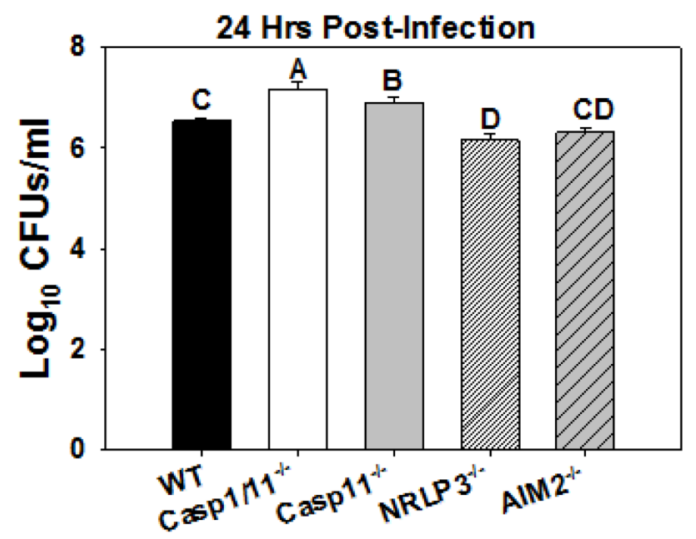

B.

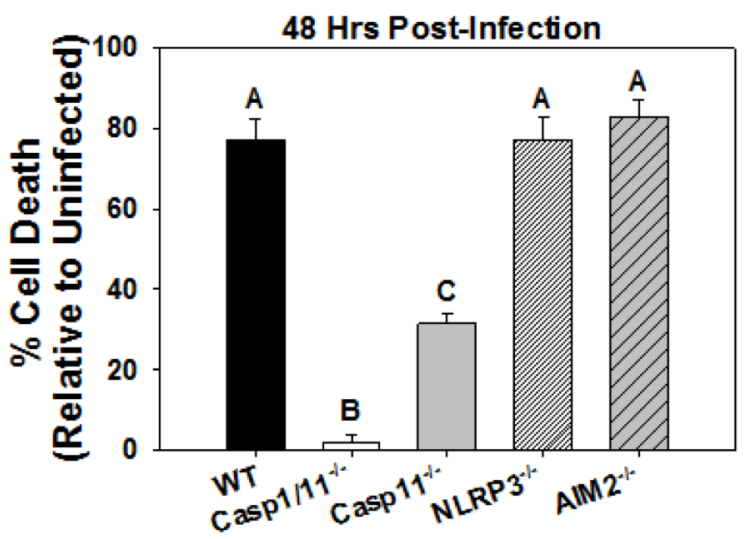

D.

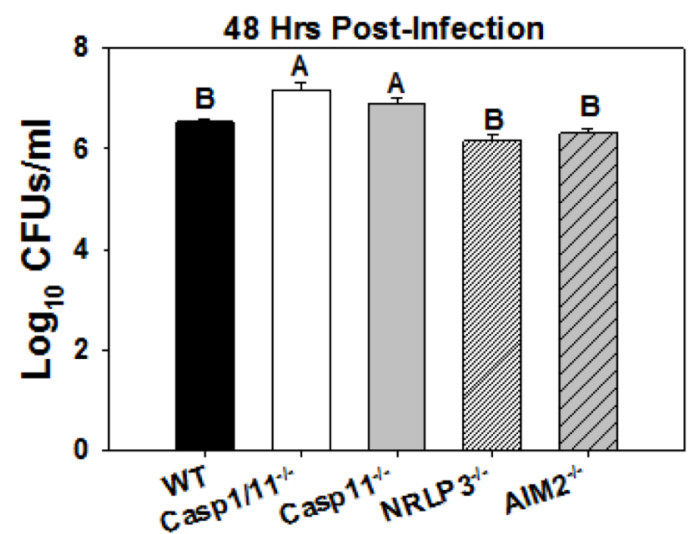

Figure 23. Brucella induces caspase-1 and caspase-11 dependent pyroptosis.

WT, caspase- $1 / 11^{-/}$, caspase- $11^{-/}$, NLRP3 $3^{-/-}$and AIM2 $2^{-/-}$BMDMs were infected with $B$. melitensis $16 \mathrm{M}$ at an $\mathrm{MOI}$ of 100 (A-D). Supernatants were harvested at 24 and 48 hours and \% cell death was determined by LDH release (A\&B). Remaining cells were lysed and Brucella loads determined (C\&D). Data are representative of 2 independent experiments (4 wells/group). Means with the same letter are not statistically different from each other as determined by ANOVA followed by Tukey's test. Error bars depict S.D. of the mean. 
A.

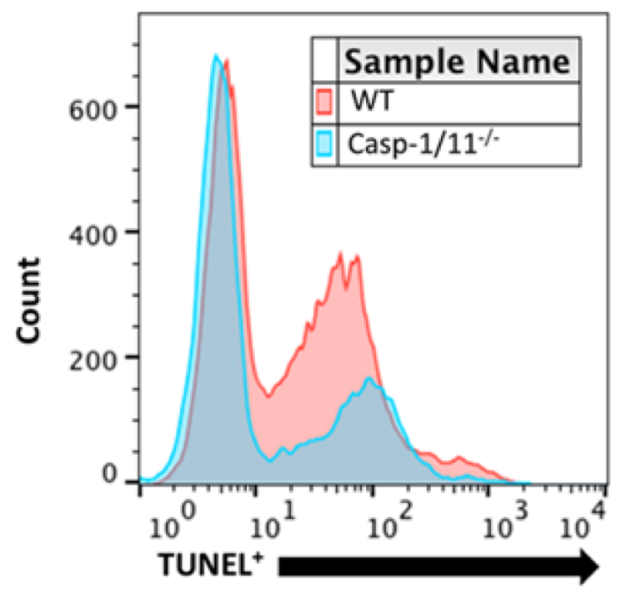

C.

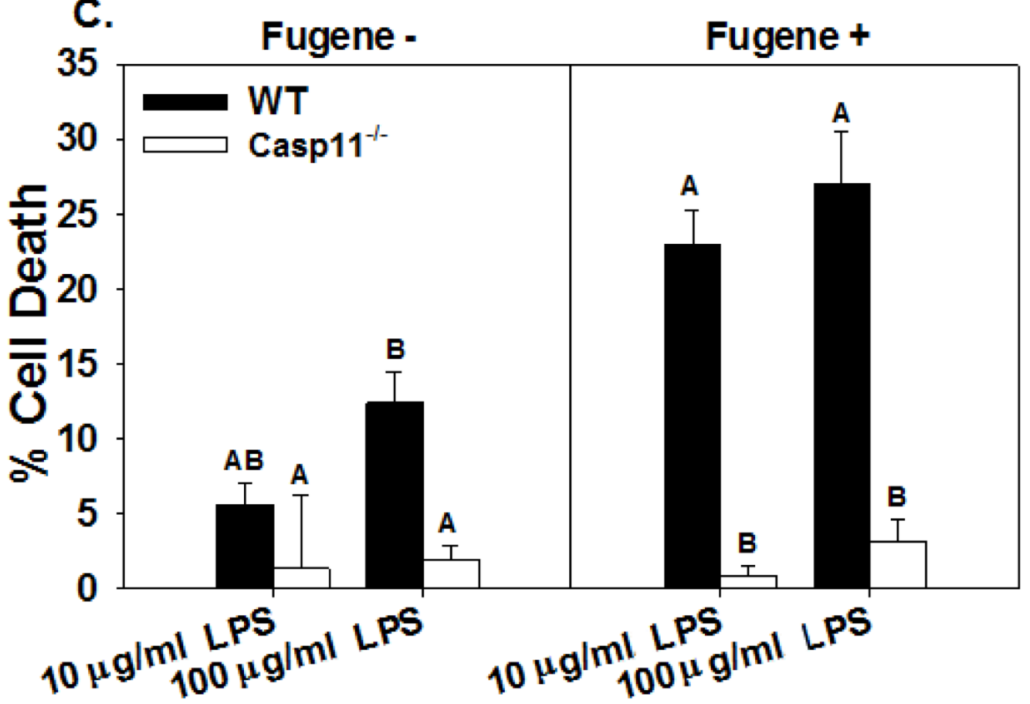

B.

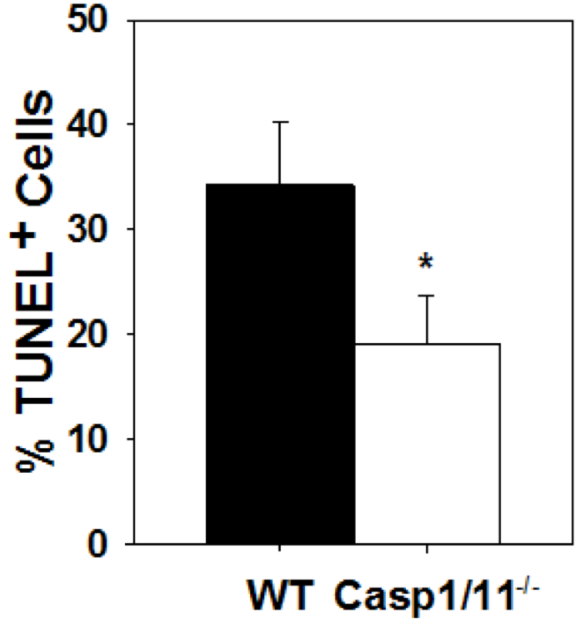

Fugene +

Figure 24. Brucella infection induces caspase-1/11-dependent cell death in vivo, and transfection of Brucella LPS induces caspase-11 dependent cell death in vitro.

WT and caspase- $1 / 11^{-/-}$mice were infected in each rear footpad with $1 \times 10^{5} \mathrm{~B}$. melitensis $16 \mathrm{M}$. Mice were euthanized at day 2 post-infection and joints were evaluated for TUNEL staining via flow cytometry. Depicted is a representative histogram of the proportion of TUNEL ${ }^{+}$cells in WT versus caspase- $1 / 11^{-1-}$ mice (A), and the percentage of TUNEL positive cells ( $n=3-5$ mice/group) in each mouse strain (B). *, $\mathrm{P}<0.05$ compared to WT mice. Data are representative of one experiment. WT or caspase- $11^{-/-}$macrophages were treated with Brucella LPS alone, or Brucella LPS packaged in Fugene. After 9 hours of culture supernatant was harvested and assessed for cell death. Data are representative of one experiment ( 3 wells/group) (C). Means with the same letter are not statistically different from each other as determined by ANOVA followed by Tukey's test. Error bars depict S.D. of the mean. 
and this phenotype was enhanced upon the addition of the transfection reagent Fugene, confirming cytosolic Brucella LPS is capable of inducing caspase-11dependent cell death (Fig 24C). These data suggest caspase-11 and caspase-1 induced pyroptosis is protective against Brucella infection.

\section{Inflammasomes do not restrain Brucella infection due to their ability to restrict host L-lactate production}

Previous studies have demonstrated that $B$. abortus thrives in glycolytic cells via $B$. abortus' ability to utilize host lactate via the Brucella L-lactate dehydrogenase gene $(I / d D)(101,225)$. However, inflammasome activation resulting in pyroptosis, releases host cell lactate dehydrogenase which can degrade L-lactate (226). Additionally, other studies suggest active caspase-1 can directly inhibit glycolysis and subsequent production of L-lactate via cleavage of glycolytic enzymes (227). To determine if caspase-1/11 was protective against Brucella via its ability to restrict L-lactate, WT and caspase- $1 / 11^{-1-}$ bone marrow derived macrophages (BMDMs) were infected with B. melitensis, and L-lactate in cell supernatant was measured. L-lactate production was induced during $B$. melitensis infection in both WT and caspase-1/11-/- macrophages; however, Llactate levels in wells containing caspase $-1 / 11^{-/-}$cells were elevated compared to WT (Fig 25A). While WT and caspase- $1 / 11^{-/-}$cells had similar rates of Brucella phagocytosis (data not shown), caspase- $1 / 11^{-/-}$BMDMs had increased Brucella burden at 48 hours post-infection (Fig 25B). In order to determine if glycolysis and subsequent production of L-lactate aided in Brucella survival, we inhibited glycolysis using 2-deoxy-glucose (2-DG) in B. melitensis infected BMDMs. At 
A.
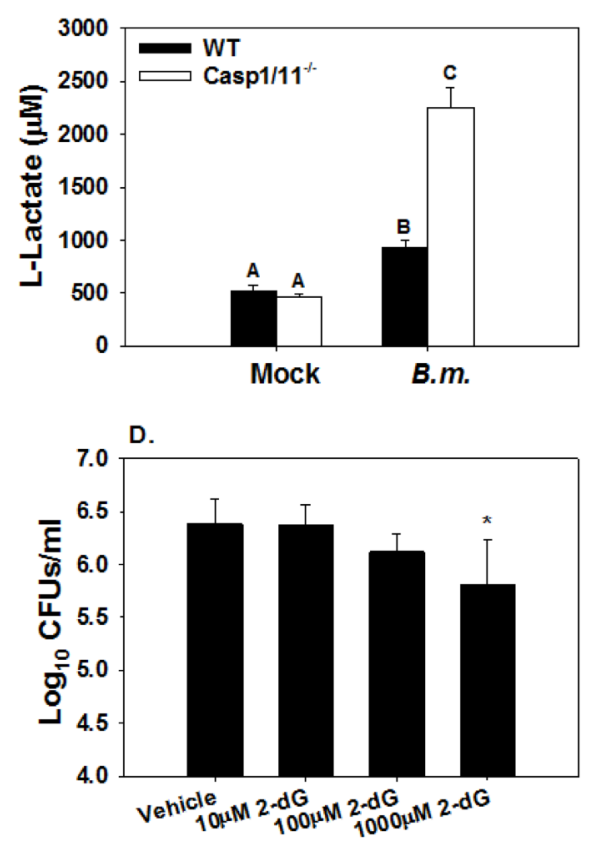

B.
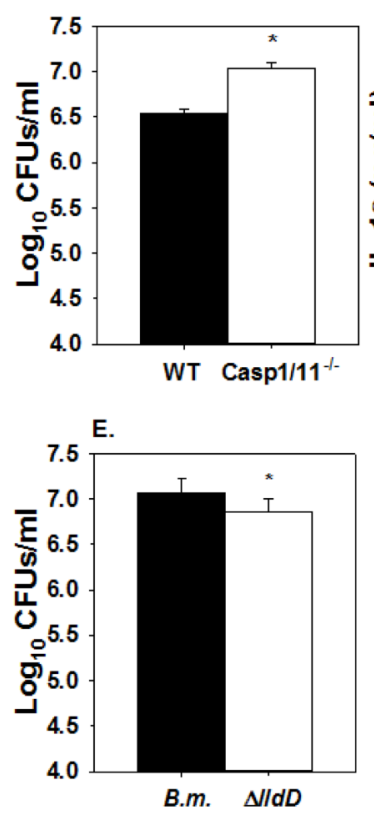

C.
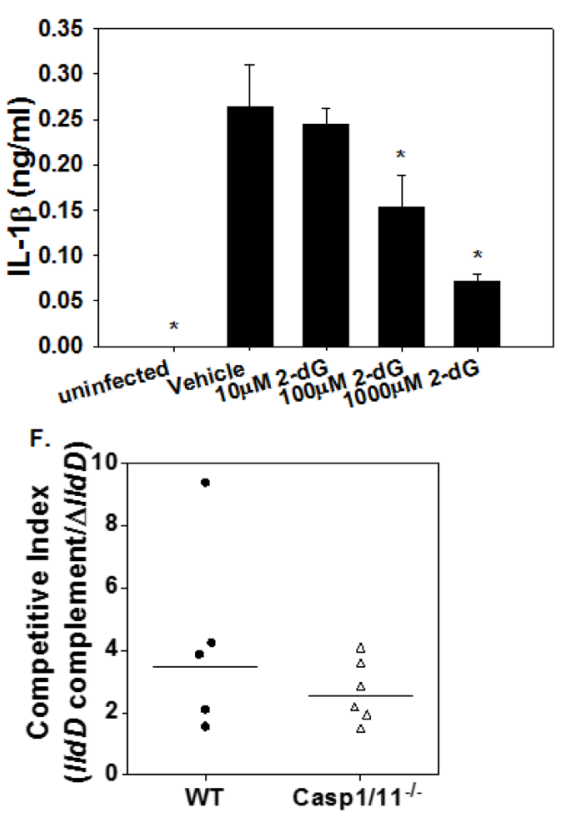

Figure 25. Increased lactate production by Caspase $-1 / 11^{-/-}$macrophages does not promote Brucella infection.

BMDM strains were infected for 6 hours with B. melitensis $16 \mathrm{M}$ at and $\mathrm{MOI}$ of 100 as described in Materials and Methods (A-F). At 48 hours post-infection Llactate was measured in cell supernatant (A). Means with the same letter are not statistically different from each other as determined by ANOVA followed by Tukey's test. At 48 hours post-infection cells were lysed and Brucella loads were determined. Data representative of 2 independent experiments with 6-8 wells/group. *, $\mathrm{P}<0.05$ compared to WT infected BMDMs (B). Some WT wells were treated with 2-DG following infection. At 48 hours IL-1 $\beta$ was measured in cell supernatants (C) and Brucella loads were determined (D). Data are representative of 2 independent experiments (3-8 wells/group) ${ }^{*}, P<0.05$ compared to WT as determined by ANOVA followed by Dunnett's test. WT BMDMs were infected with either B. melitensis or B. melitensis $\Delta / l d D$. At 48 hours post-infection intracellular Brucella burdens was determined. Data are representative of one of two independent experiments (6 wells/group) ${ }^{*}, P<0.05$ compared to WT B. melitensis $(\mathrm{E})$. BMDMs were co-infected with a 1:1 ratio of $\Delta / / d D$ and an $\Delta / / d D$ complemented strain of $B$. melitensis. 48 hours post-infection cells were lysed and the competitive index was determined in each BMDM strain. Data are representative of two independent experiments 5 wells/group (F). Error bars depict S.D. of the mean. 
$1000 \mu \mathrm{M}, 2-\mathrm{DG}$ reduced both intracellular $B$. melitensis and IL-1 $\beta$ release, while $100 \mu \mathrm{M}$ 2-DG only inhibited IL-1 $\beta$ release (Fig 25C\&D). Thus, 2-DG may inhibit inflammasome-dependent IL-1 $\beta$ production, and intracellular survival of Brucella. Interestingly, Brucella infected mice treated with 2-DG exhibited unaltered Brucella loads, but reduced swelling, relative to vehicle treated mice (Fig 26A\&B), suggesting 2-DG may also inhibit inflammasome dependent inflammation in vivo.

As an alternative to targeting host cell metabolism, we made a $B$. melitensis L-lactate dehydrogenase mutant $(\Delta / / d D)$, to inhibit Brucella's ability to utilize free L-lactate (101). $\Delta / / d D$ was attenuated in WT macrophages compared to WT $B$. melitensis, (Fig 25E). Therefore, we investigated whether IIdD conferred a greater competitive advantage in caspase-1/11 $1^{-/}$cells than in WT macrophages due to enhanced lactate production. Thus, we performed competition experiments between $\Delta / l d D$ and a complemented $\Delta / l d D$ strain in both WT and caspase $1 / 11^{-/-}$ cells. We found that our complemented strain out-competed our mutant in both WT and caspase-1/11/- macrophages; however, the competitive index of the IIdDcomplemented strain compared to $\Delta / l d D$ was not significantly different between macrophage strains. These data indicates caspases $1 / 11$ does not restrict Brucella survival via their ability to reduce L-lactate production (Fig $25 \mathrm{~F}$ ).

\section{IFN- $\gamma$ restrains Caspase-1/11-induced joint inflammation}

We previously demonstrated IFN-ץ prevents inflammation and joint infection in a systemic model of Brucella-induced arthritis (70). In addition, we have found that caspase-1 dependent IL-18 induces protective IFN- $\gamma$ in response to 


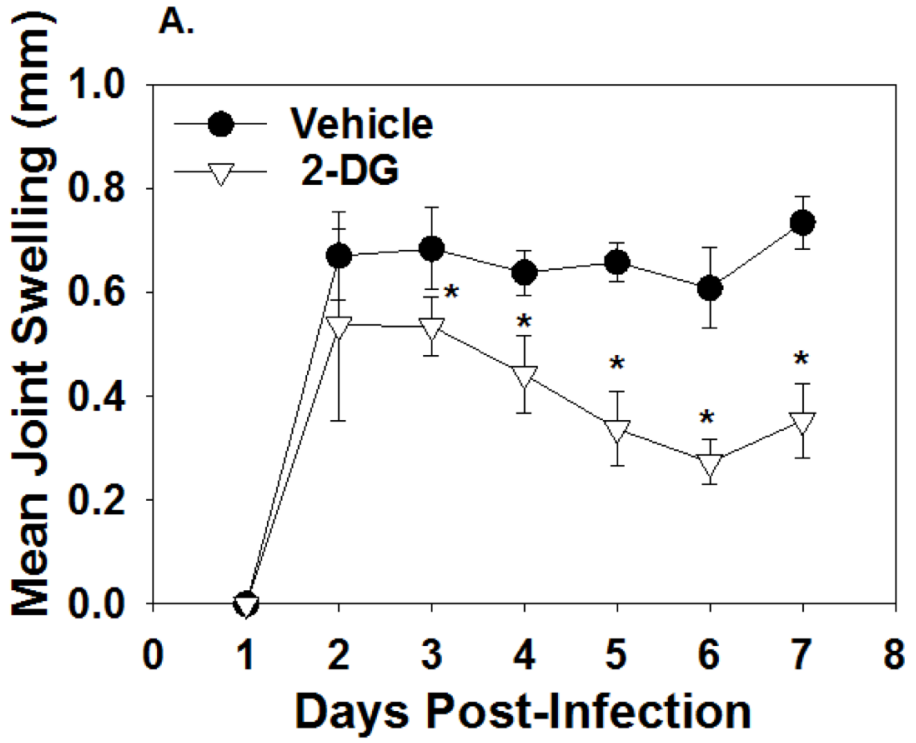

B.

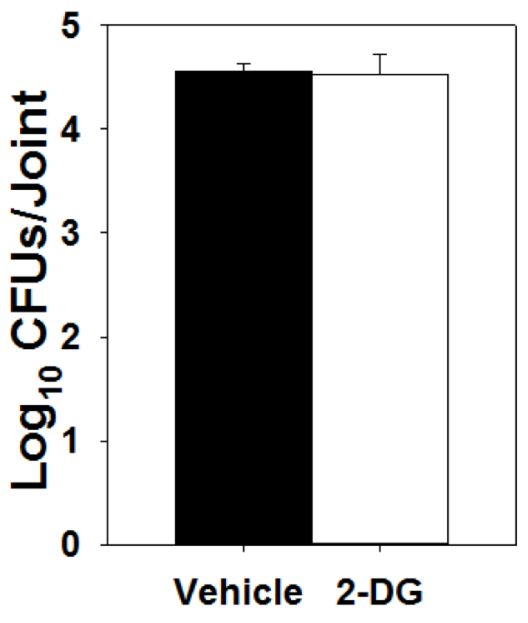

Figure 26. 2-DG prevents swelling during Brucella infection.

Mice were infected in both rear footpads with $B$. melitensis $16 \mathrm{M}$ and were treated i.p. with 2-DG on days 1 and 3 post-infection. Swelling was recorded over time (A) and joint Brucella burdens were measured at day 7 post-infection (B). Data is reported as the difference in swelling from the start of 2-dG treatment (day 1 postinfection). Data is representative of one experiment 4-5 mice/group. ${ }^{*}, \mathrm{P}<0.05$ compared to WT mice. Error bars depict S.D. of the mean. 
Francisella tularensis (228). Therefore, the effects of inflammasomes on IFN-Y production in Brucella-induced arthritis were investigated. At day 2 post-Brucella infection, IFN-y levels were elevated in WT joints compared to caspase- $1 / 11^{-1-}$ mice (Fig 18B). IL-18 deficiency also resulted in significantly reduced joint IFN-y levels, suggesting caspase-1/11-dependent IL-18 could control Brucella colonization in the joint (Fig 27). To investigate whether IFN- $\gamma$ was protective during focal infection and if this protection was inflammasome dependent, we treated WT and caspase-1/11 ${ }^{-/}$mice with anti-IFN-y or an isotype control. IFN-y neutralized WT mice displayed markedly enhanced joint swelling by day 3 post-infection and everyday thereafter compared to all other groups (Fig 28A). This severe joint swelling was associated with an increase in myeloid cell recruitment including a robust increase in neutrophils and macrophages (Fig 29A\&B). In contrast, IFN- $\gamma$ neutralization in caspase-1/11 $1^{-/}$mice did not result in enhanced joint swelling; rather, it reduced swelling at days 3-4 post-infection. Histological analysis at day 7 post-infection demonstrated caspase- $1 / 11^{-/-}$-anti-IFN- $\gamma$ treated mice had similar joint inflammation scores as WT and caspase-1/11 ${ }^{-/-}$IgG-treated mice. In contrast, WT-anti-IFN-y treated mice had significantly greater inflammation scores compared to all other groups examined (Fig 28B\&C). IL-1 $\beta$ was also elevated in joint homogenates of WT-anti-IFN- $\gamma$ treated mice compared to all other groups, possibly indicating increased caspase-1 activation in the absence of IFN- $\gamma$ (Fig 30). At day 7 post infection, we found mice deficient in either IFN- $\gamma$ or caspase-1/11 had increased Brucella burdens; however, caspase- $1 / 11^{-/-}$mice neutralized of IFN- $\gamma$ had higher Brucella joint colonization than WT mice neutralized of IFN- $\gamma$. 


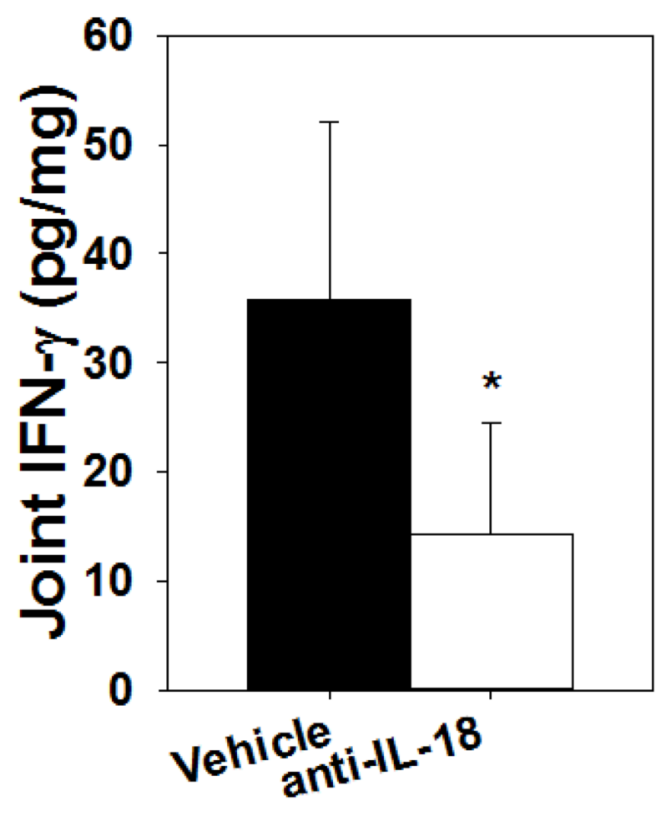

Figure 27. IL-18 contributes to IFN-y production in Brucella infected joints. WT mice were treated with anti-IL-18 or a vehicle control, then mice were infected in each rear footpad with $B$. melitensis $16 \mathrm{M}$. Mice were euthanized at day 2 post-infection and joint IFN- $\gamma$ was determined ( $n=5$ mice/group) Data are representative of one experiment. * $\mathrm{P}<0.05$ compared to vehicle-treated mice. Error bars depict S.D. of the mean. 
A.

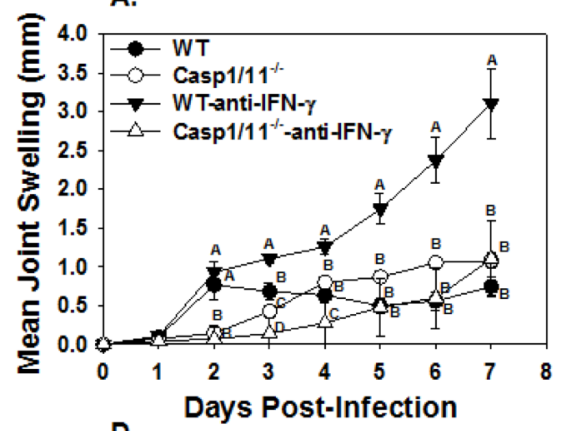

D.

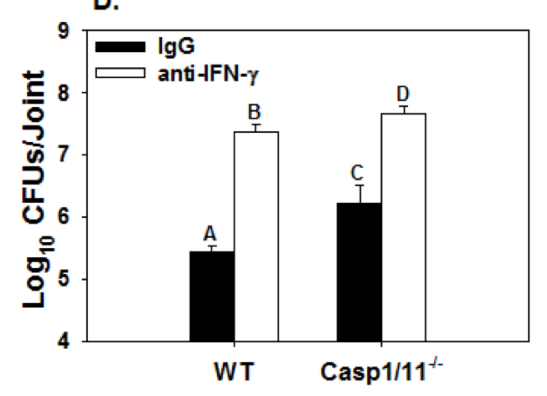

B.
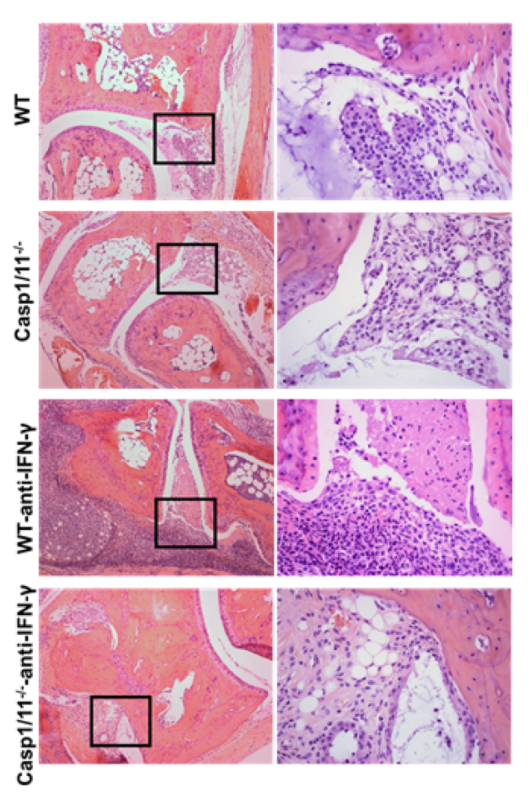

c.

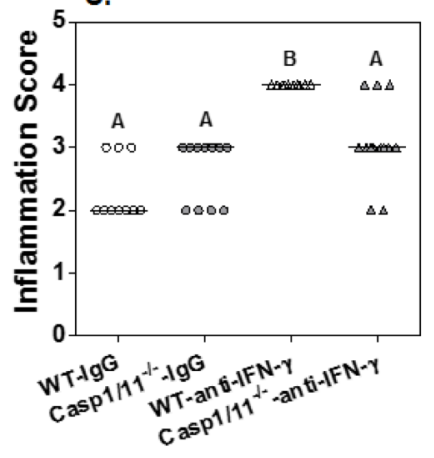

Figure 28. IFN- $y$ deficiency results in severe caspase-1/11-dependent inflammation.

WT and caspase- $1 / 11^{-/-}$mice were treated with anti-IFN-y or an isotype control, then infected in both rear footpads with $1 \times 10^{5} \mathrm{~B}$. melitensis $16 \mathrm{M}$. Joint swelling was measured throughout the experimental time course (A). Mice were euthanized at day 7 post-infection and H\&E staining was conducted and slides were scored for inflammation (B\&C). Depicted are representative images (100X) from each group with amplified boxed regions (400X) displayed to their right (B). Joint Brucella burdens were also enumerated 7 days post-infection (D). Data are representative of 3 independent experiments with 3-5 mice/group except for panel C, in which data was combined from 2 experiments with 10-13 mice/group. Means/medians with the same letter are not statistically different from each other as determined by ANOVA followed by Tukey's test or ANOVA on ranks. Error bars depict S.D. of the mean. 
A.

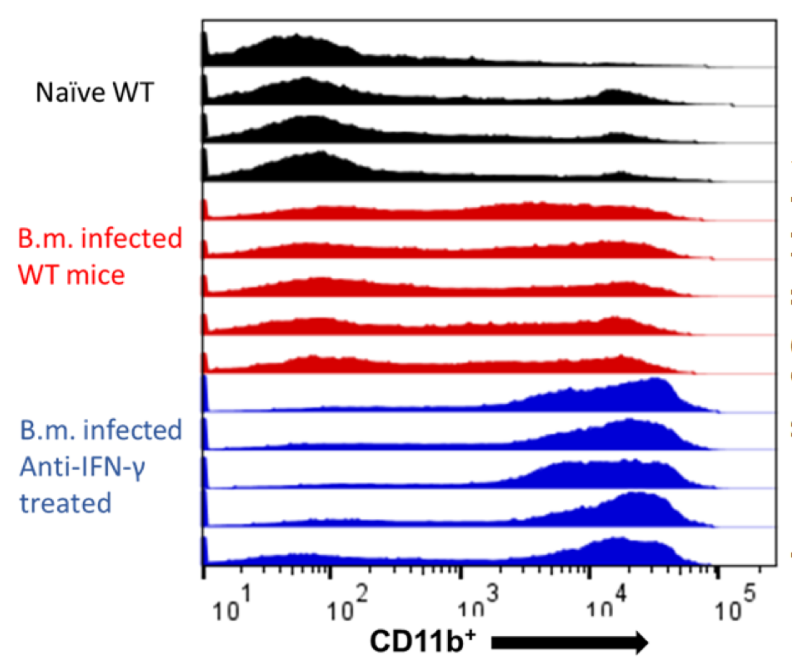

B.

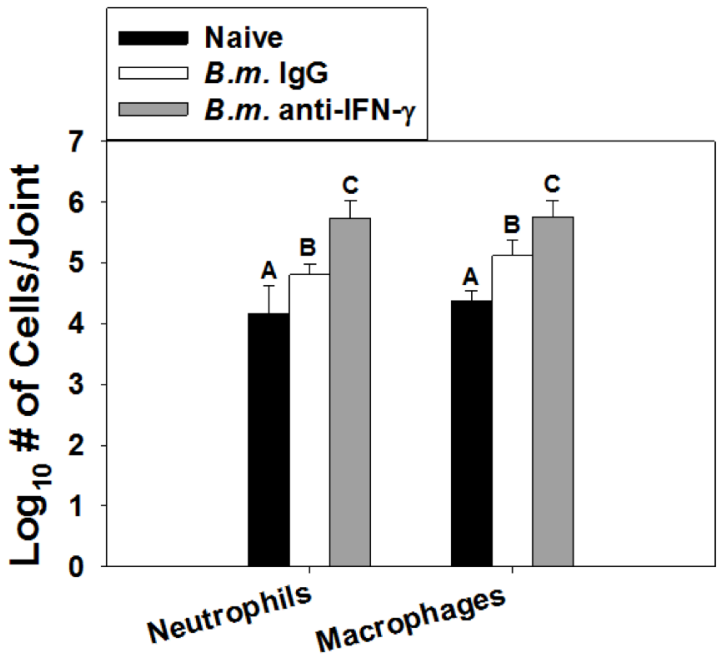

Figure 29. IFN- $\gamma$ deficiency leads to increased myeloid cell recruitment in Brucella infected joints.

Select mice were treated with an anti-IFN- $\gamma$ depleting antibody. Then, mice were infected in each rear footpad with $1 \times 10^{5} \mathrm{~B}$. melitensis $16 \mathrm{M}$. Mice were euthanized at 7 days post-infection and flow cytometry was conducted on joints from infected mice and naïve controls (A\&B). Depicted are histograms of $\mathrm{CD} 11 \mathrm{~b}^{+}$cells in individual mice (A), and bar charts indicating the average number of neutrophils or macrophages in each group ( $n=4-5$ mice/group) (B). Means with the same letter are not statistically different from each other as determined by ANOVA followed by Tukey's test. Error bars depict S.D. of the mean. Data are representative of one experiment. 


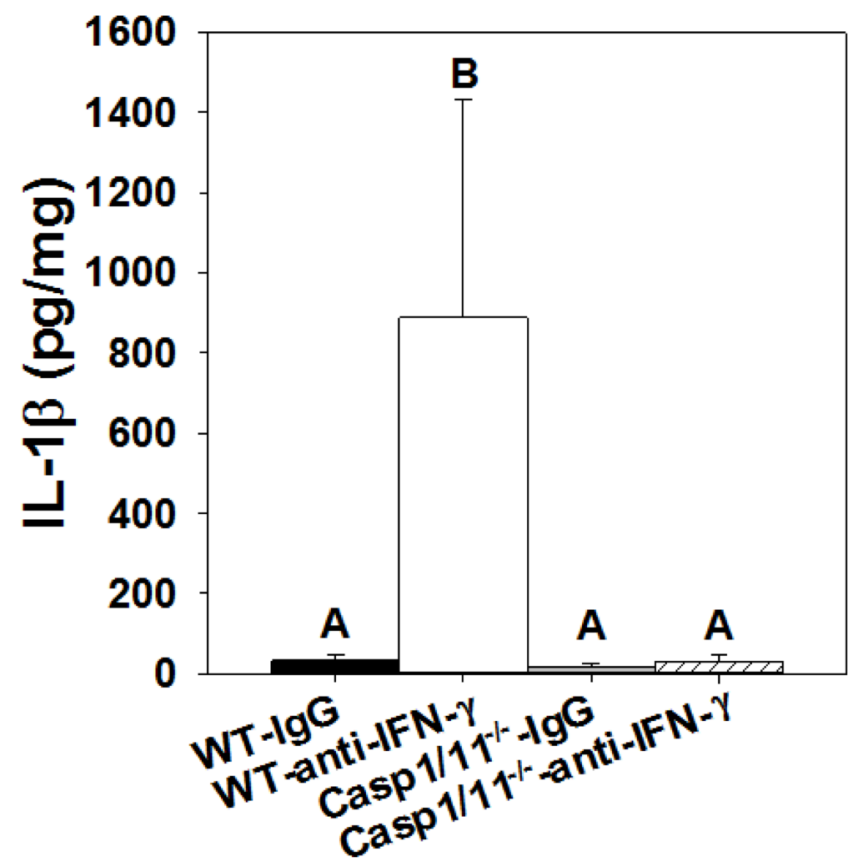

Figure 30. IFN- $\gamma$ deficiency leads to increased inflammasome dependent $\mathrm{IL}-1 \beta$ in vivo.

WT and caspase-1/11 $11^{-/}$mice were treated with anti-IFN-y or an isotype control, then infected in both rear footpads with $1 \times 10^{5} \mathrm{~B}$. melitensis $16 \mathrm{M}$. Mice were euthanized at day 7 post-infection. IL-1 $\beta$ was measured from joint homogenates (4-5 mice/group). Data are representative of 2 independent experiments. Means with the same letter are not statistically different from each other as determined via ANOVA followed by Tukey's test. Error bars depict S.D. of the mean. 
This indicates that while IL-18 induced IFN- $\gamma$ may be partially responsible for inflammasome-dependent protection, caspase-1/11 has additional IFN- $\gamma$ independent means of restricting Brucella infection (Fig 28D).

\section{Adaptive immune cell IFN- $\gamma$ production contributes to Brucella clearance}

We have previously demonstrated that T-cells make IFN- $\gamma$ upon footpad infection with Brucella. Additionally, NK cells can be important producers of IFN- $\gamma$ and $\gamma \delta$ T-cells have been reported to make IFN- $\gamma$ upon Brucella infection (163, 229). In order to determine what cells were making protective IFN- $\gamma$ in our model we neutralized NK cells or $\gamma \delta$ T-cells from WT mice (Fig 31A\&B). Both NK-cell neutralized mice and $\gamma \delta$ T-cell neutralized mice displayed similar swelling and joint Brucella burdens at day 7 post-infection, indicating NK-cells or $\gamma \delta$ T-cell are not essential for protective IFN- $\gamma$ in our model. Similarly, we compared control WT and Rag $1^{-/-}$mice to those neutralized of IFN- $\gamma$ and evaluated joint CFUs at day 7 postinfection. Rag $1^{-/-}$mice only had an effect on joint Brucella burdens in mice sufficient in IFN- $\gamma$, indicating adaptive immune cell IFN- $\gamma$ is important for joint Brucella clearance (Fig 31 C\&D).

\section{NLRP3 and Caspase-11 are partially responsible for inducing inflammation in IFN- $\gamma$ deficient mice.}

In order to identify what inflammasomes contribute to arthritis and if these phenotypes are altered in the absence of IFN- $\gamma$, we treated WT and NLRP3 ${ }^{-1-}$ mice 
A.

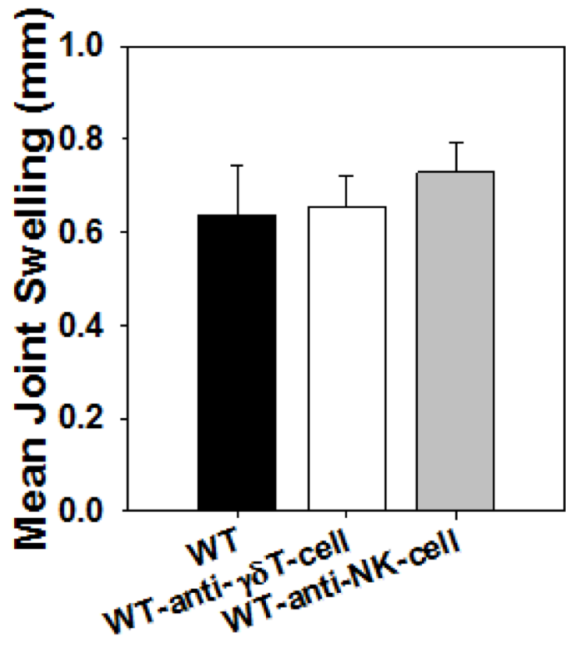

c.

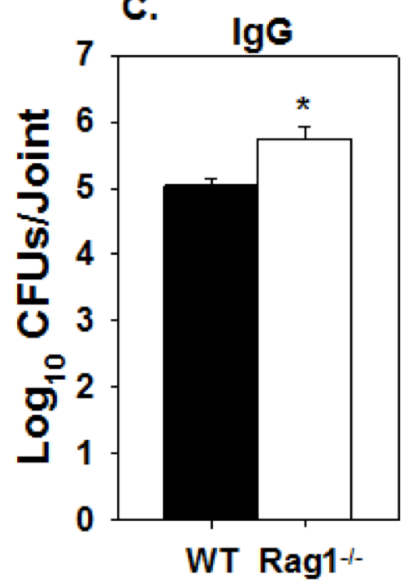

B.

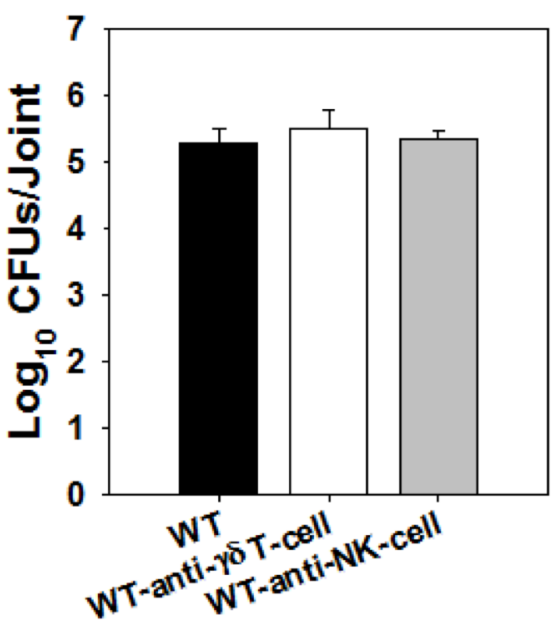

D.

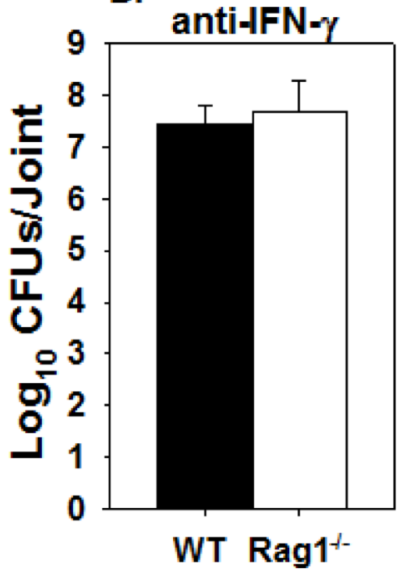

Figure 31. Adaptive immune cell IFN- $\gamma$ production is important for Brucella clearance.

Mice were treated i.p. with anti-NK-cell antibody or anti- $\gamma \delta \mathrm{T}$-cell antibody 1 day prior and 3 days after infection in both rear footpads with $1 \times 10^{5}$ B. melitensis $16 \mathrm{M}$. Joint swelling $(\mathbf{A})$ and CFUs (B) were evaluated 7 days post-infection. Data are representative of one experiment 5 mice/group. In a separate study WT and Rag ${ }^{-1-}$ mice were treated with anti-IFN- $\gamma$ or an isotype control, then infected in each rear footpad with $1 \times 10^{5} \mathrm{~B}$. melitensis $16 \mathrm{M}$. At 7 days postinfection mice were euthanized and joint CFUs were enumerated (C\&D). Data are representative of 1 experiment 3-5 mice/group. *, $P<0.05$ compared to WT mice. Error bars depict S.D. of the mean. 
with anti-IFN-y or an isotype control. Only a mild reduction in swelling was seen in the isotype-treated $\mathrm{NLRP}^{-/-}$mice compared to $\mathrm{WT}$ isotype controls at day 2 post infection. Interestingly, in the absence of IFN- $\gamma$, NLRP3 deficiency reduced joint swelling throughout infection (Fig 32A). We also examined AIM2's role in inducing joint inflammation; however, AIM2 appeared to be dispensable for the induction of swelling both in the presence and absence of IFN- $\gamma$ (Fig 32B). Swelling in IFN- $\gamma$ neutralized mice was partially attributed to the non-canonical inflammasome, as caspase- $11^{-/-}$-anti-IFN- $\gamma$ treated mice had reduced joint swelling at days 5 and 7 compared to WT, IFN- $\gamma$ neutralized mice (Fig 32C).

IFN- $\gamma$ induces the upregulation of iNOS, and iNOS deficiency enhances joint inflammation

To explain why NLRP3 plays a major role in swelling only in the absence of IFN- $\gamma$ and why inflammation in IFN- $\gamma$-deficient mice is caspase1/11-dependent, we considered IFN- $\gamma$ may be inhibiting inflammasome activation. Others have found that IFN-y induced nitric oxide (NO) can inhibit NLRP3 and caspase-1 (161, 230, 231). Thus, we hypothesized the NO producing enzyme, inducible nitric oxide synthase (iNOS), may be enhanced by IFN- $\gamma$ during Brucella infection of the joint. Hence, we examined iNOS transcript in joints from infected mice treated with antiIFN- $\gamma$ or an isotype control. At day 2 post-infection, isotype control mice displayed increased iNOS transcript compared to IFN- $\gamma$-deficient mice (Fig 33A). IFN- $\gamma$ was also critical for iNOS mRNA expression at later time points (data not shown). To determine if iNOS was protective against swelling, ankle widths of iNOS deficient 
A.

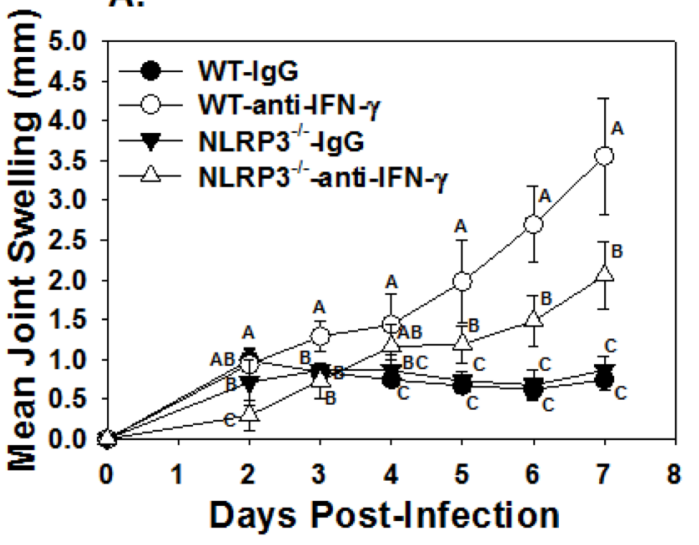

B.

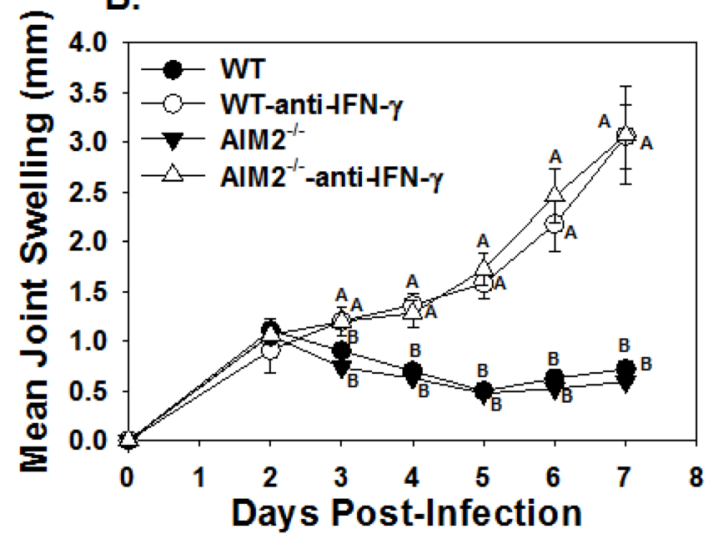

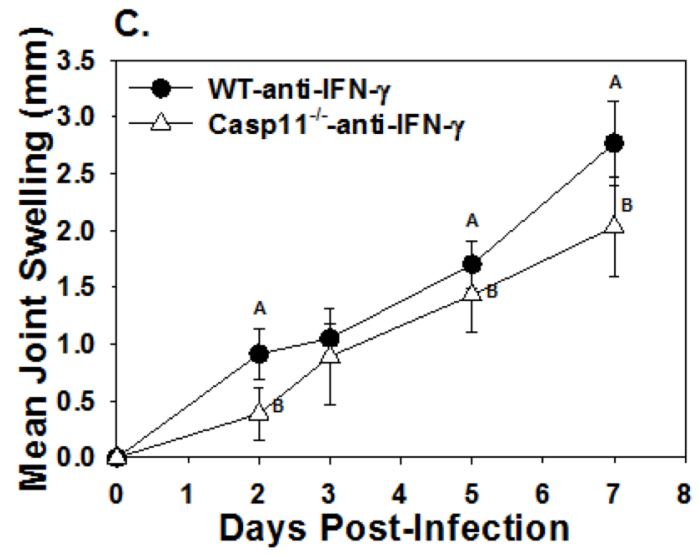

Figure 32. NLRP3 and Caspase-11 induce inflammation in the absence of IFN- $\gamma$.

WT and NLRP3 ${ }^{-/}$mice were treated with anti-IFN- $\gamma$ or an isotype control, then infected in their rear footpads with $1 \times 10^{5} \mathrm{~B}$. melitensis $16 \mathrm{M}$. Rear ankle joint swelling was measured overtime. Data are combined from 2 independent experiments (9-10 mice/group total) (A). WT and AIM2 ${ }^{-/-}$mice were treated with anti-IFN- $\gamma$ or sPBS, then infected in the rear footpads with $1 \times 10^{5} B$. melitensis and ankle swelling was monitored overtime. Data are representative of 2 independent experiments (4-5 mice/group) (B). WT and Caspase $-11^{-/}$mice were both treated with anti-IFN- $\gamma$ then infected with $1 \times 10^{5}$ B. melitensis $16 \mathrm{M}$ in both rear footpads. Ankle swelling was evaluated for 7 days. Data are combined from 3 separate experiments (13-18 mice/group total) (C). Means with the same letter are not statistically different from each other as determined by ANOVA followed by Tukey's test. Error bars depict S.D. of the mean. 

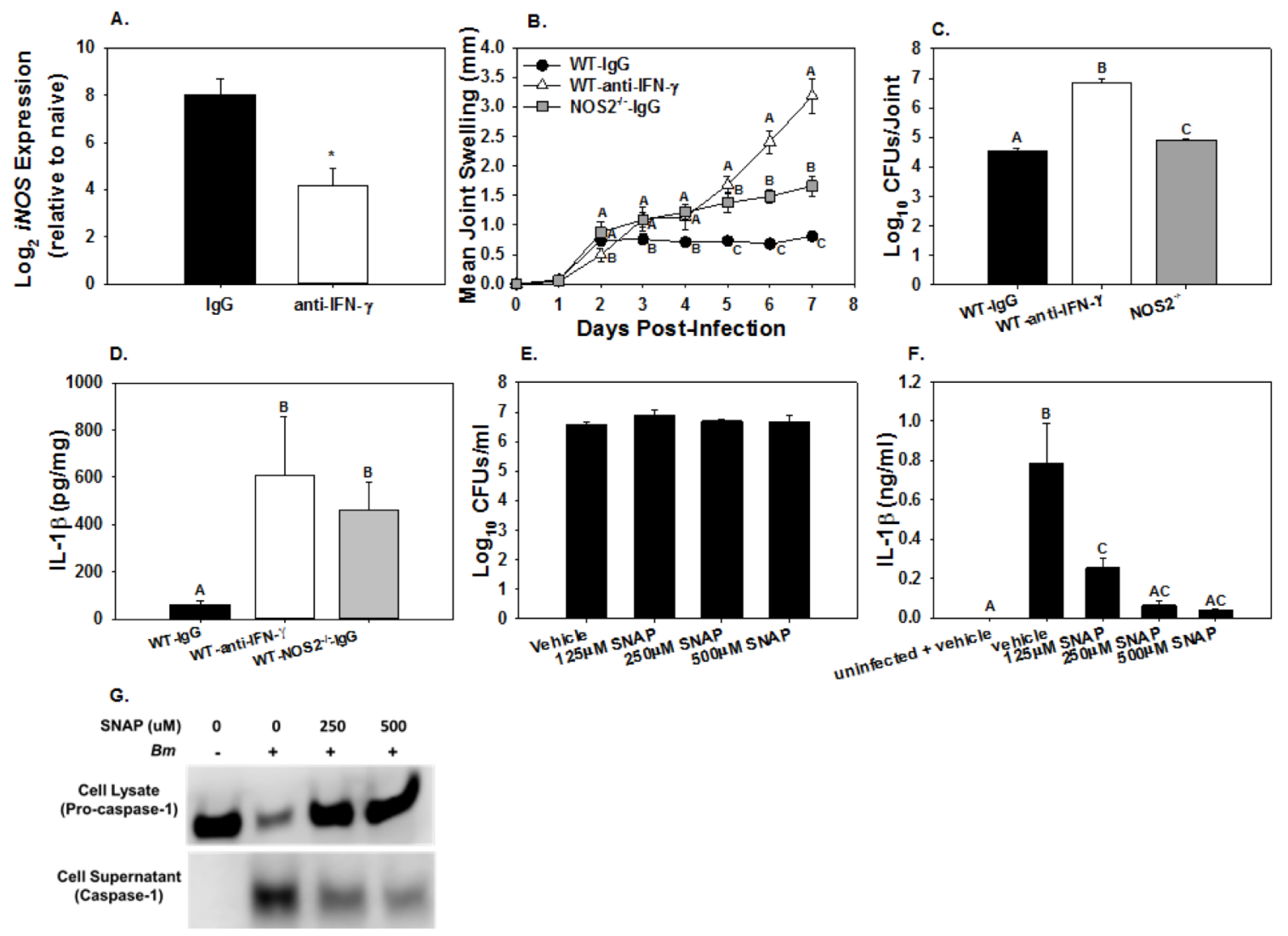

Figure 33. Nitric oxide inhibits Brucella-induced caspase-1 activation and IL-1 $\beta$ production.

WT mice were treated with either anti-IFN- $\gamma$ or an isotype control, then mice were infected in the rear footpads with $1 \times 10^{5} \mathrm{~B}$. melitensis $16 \mathrm{M}$. Mice were euthanized at day 2 post-infection along with naïve mice and RNA was isolated from the ankle joints and relative iNOS transcript was then determined. Data are representative of one experiment (3-4 mice/group). *, P<0.05 compared to WT mice (A). In a separate experiment, WT mice received anti-IFN- $\gamma$ while other WT mice along with $\mathrm{NOS}^{-1-}$ mice received an isotype control. Mice were infected in the rear footpads with $1 \times 10^{5} \mathrm{~B}$. melitensis $16 \mathrm{M}$ and monitored for swelling (B). Mice were euthanized at day 7 post-infection and joint CFUs and IL-1 $\beta$ were enumerated (C\&D). Data are representative of one experiment (4-5 mice/group). WT BMDMs were infected with B. melitensis $16 \mathrm{M}$ at an MOI of 100 for 6 hours. Media containing increasing doses of SNAP was added immediately after infection. 24 hours post-infection intracellular Brucella burdens were enumerated (E) and IL-1 $\beta$ levels in cell supernatants were determined (F). Cell supernatants and cell lysates were also subjected to western blot analysis (G). Data is representative of 2 independent experiments with 3-5 wells/group. Means with the same letter are not statistically different from each other as determined by ANOVA followed by Tukey's test. Error bars depict S.D. of the mean. 
mice $\left(\mathrm{NOS2}^{-{ }^{-}}\right.$) were evaluated following Brucella infection. NOS2 ${ }^{-/-}$mice displayed increased swelling relative to WT-IgG mice, but reduced swelling compared to WTanti-IFN- $\gamma$ treated animals (Fig 33B). At day 7 post-infection, iNOS deficiency also resulted in increased joint Brucella burdens relative to WT mice, but this difference was not as pronounced as that observed with anti-IFN-y treatment (Fig 33C). $\mathrm{NOS}^{-/-}$mice also had similarly elevated joint IL-1 $\beta$ as WT IFN- $\gamma$ neutralized mice (Fig 33D), despite bacterial loads nearly 100 -fold lower, indicating NO could limit inflammation by mechanisms beyond controlling Brucella burdens. Similarly, mice that received the iNOS inhibitor, aminoguanidine hemisulfate salt (AGHS), in their drinking water had increased joint inflammation and Brucella burdens at day 18 post-infection relative to controls (Fig 34A\&B). We also observed IL-1 $\beta$ was upregulated in the joints of AGHS treated animals compared to animals receiving regular drinking water (Fig 34C).

\section{Nitric oxide inhibits Brucella-induced caspase-1 activation}

To determine if NO can directly inhibit inflammasome activation during Brucella infection, BMDMs were infected with $B$. melitensis then treated with increasing doses of the NO donor, S-nitroso-N-acetyl-penicillamine (SNAP). In vitro media conditions and infection times were optimized so that intracellular Brucella loads were similar in WT and SNAP treated wells at the time of cell lysate and supernatant harvest (Fig 33E). Analysis of IL-1 $\beta$ in cell supernatants at 24 hours post-infection demonstrated reduced IL-1 $\beta$ release upon administration of SNAP (Fig 33F). Western blot analysis confirmed that caspase-1 is activated by 
A.

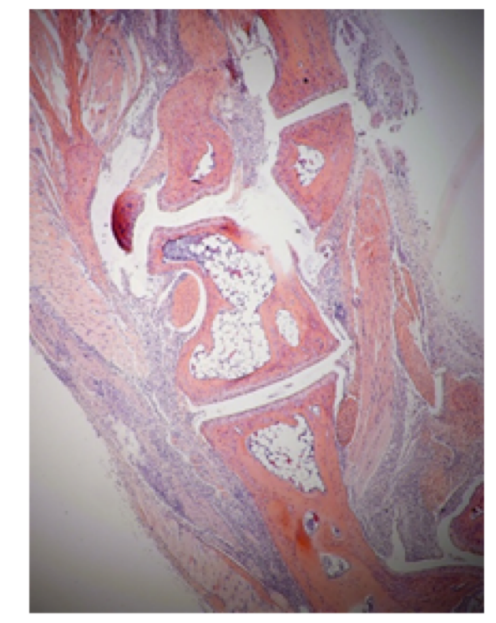

B.

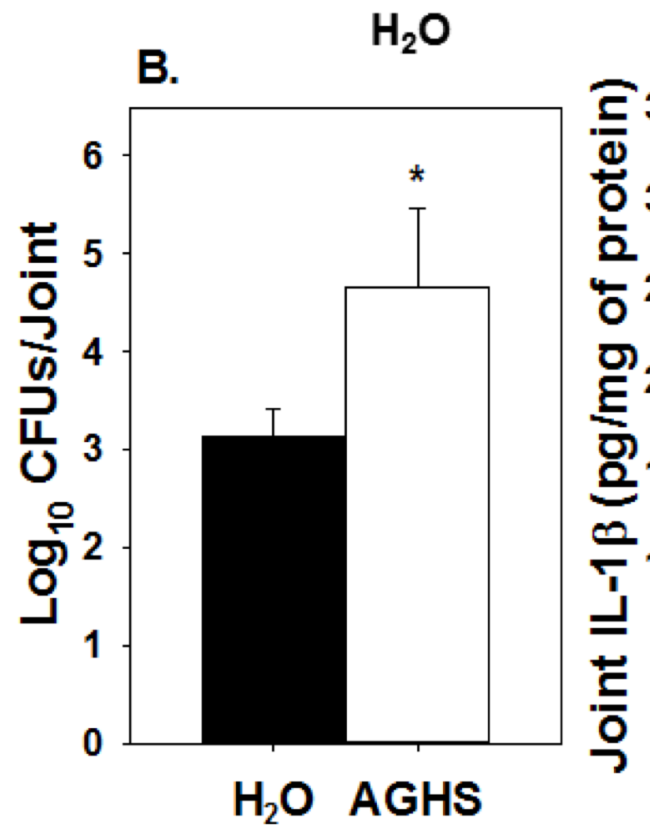

$\mathrm{H}_{2} \mathrm{O}$ AGHS

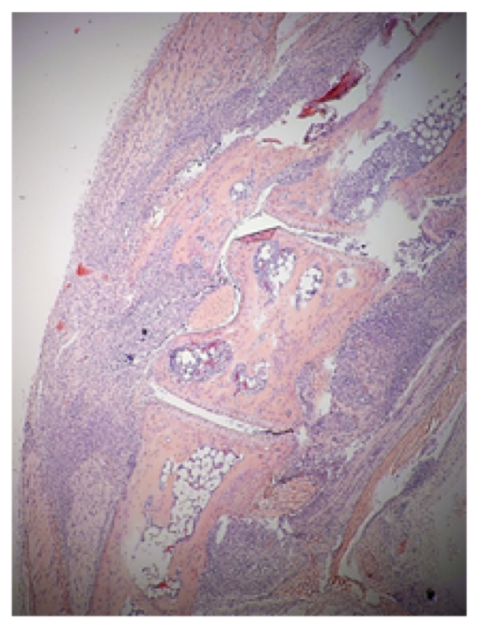

AGHS
C.

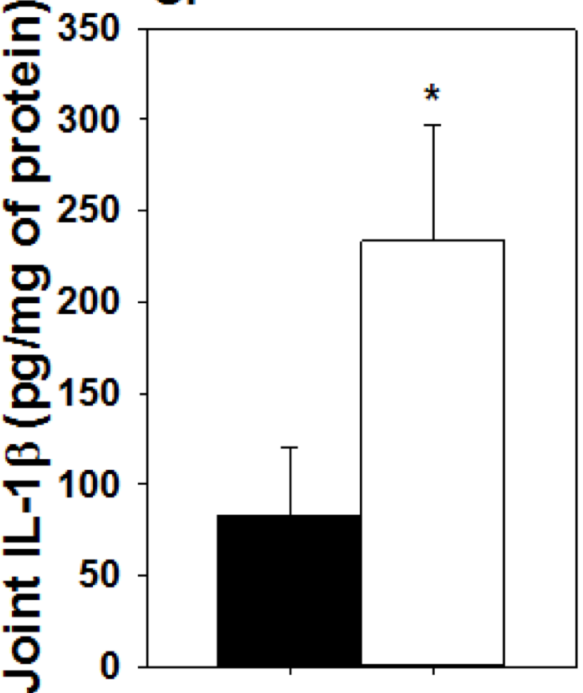

$\mathrm{H}_{2} \mathrm{O}$ AGHS

Figure 34. In vivo inhibition of iNOS with AGHS results in enhanced joint inflammation.

Some mice were given drinking water containing $2.5 \%$ AGHS while others were given normal drinking water. All mice were infected in each rear footpad with $1 \times 10^{5}$ B. melitensis $16 \mathrm{M}$. Animals were euthanized at day 18 post-infection and H\&E staining was conducted. Representative images (100x) from mice receiving normal drinking water or AGHS are depicted (A). Brucella burdens (B) and IL-1 $\beta$ (C) were also evaluated in joint homogenates ( $n=4-5$ mice/group). *, $\mathrm{P}<0.05$ compared to mice receiving normal drinking water. Error bars depict S.D. of the mean. Data are representative of one experiment. 
Brucella infection, as cleaved caspase-1 was found in the cell supernatant of infected but not uninfected macrophages. Furthermore, supernatants from Brucella infected cells treated with SNAP contained reduced activated caspase-1 compared to vehicle treated (Fig 33G).

\section{DISCUSSION}

Globally brucellosis is a prevalent zoonotic disease and Brucella-induced arthritis is the most common focal complication of human brucellosis, occurring in $40-80 \%$ of patients $(56,57)$. Here we investigated the role of inflammasomes in a murine model of Brucella induced arthritis. We show that inflammasomes initiate inflammation and arthritis in mouse ankle joints. Changes in articular inflammatory responses due to inflammasomes at day 2-post-infection were not dependent on joint Brucella burden, as all mouse genotypes examined in this study had similar joint Brucella loads at this time. Thus, day 2 was used to determine differences in initiation of joint inflammation.

Caspase- 1 and caspase- 11 both initiated a joint inflammatory response at day 2 post-Brucella footpad infection. Inflammasomes play a major role in inducing pathology in rheumatoid arthritis due to their ability to process IL-1 and IL-18 into their active forms $(136,217)$. Indeed, caspase-1/11 dependent inflammation was partially reliant on IL-18, while only modest inflammatory effects of IL-1 were observed. Although inflammasomes did not influence Brucella burdens early in infection we did uncover an antimicrobial role for caspase-1 and caspase-11 beyond day 6 post-infection. Caspase-1/11, ASC, AIM2 and NLRP3 have all been 
shown to be protective against systemic Brucella abortus colonization 4 weeks following i.p. inoculation (117), but nothing is known about caspase-11's antimicrobial role. Here we demonstrated caspase-1/11 reduced joint Brucella loads by roughly one log and $\sim 1 / 3$ of this phenotype was attributed to caspase- 11 . Caspase-11 traditionally recognizes enterobacteria LPS with hexa or pentaacylated lipid A $(232,233)$. Brucella's LPS structure is known to be highly immunoevasive in part due to its hepta-acylated lipid A, core oligosaccharide and its O-polysaccharide (224). Here we demonstrated caspase-11 contributes to joint inflammation, and both live Brucella and Brucella LPS induce caspase-11dependent cell death. Similarly, Acinetobacter baumannii, another Gram-negative bacterium with hepta-acylated lipid A, has also been shown to activate caspase11, suggesting caspase-11's repertoire of lipid A recognition may be more diverse than once thought $(234,235)$.

The bactericidal activity of caspase- 1 and caspase- 11 may be in part due to their ability to induce pyroptosis. This inflammatory form of cell death can damage intracellular bacteria and render them confined to pore-induced intracellular traps thereby allowing bacteria to be easily killed via efferocytosis (215). In Brucella infected macrophages, we observed caspase-1 and caspase-11 dependent cell death, suggesting infected macrophages die via pyroptosis. Wells with reduced cell death contained increased Brucella burdens per well, indicating pyroptosis may protect the host by eliminating macrophages as a reservoir of Brucella infection. This was surprising, as canonically Brucella is thought to inhibit the death of host cells (154). Additionally, we demonstrated that transfection of 
macrophages with $B$. abortus S19 LPS results in induction of caspase-11 dependent cell death. To our knowledge this is the first report of caspase-11 recognizing Brucella LPS, although one study did demonstrate chemical inhibition of caspases 4 and 5 (human homologues of murine caspase-11) reduces Brucella LPS-induced upregulation of Annexin $V$ on human neutrophils (236). DAMPs generated by caspase-11 dependent pyroptosis can activate NLRP3 and in turn caspase-1 (237). NLRP3 did not influence joint Brucella burdens, but caspase-11 was protective against Brucella colonization. Similarly, in our in vitro studies caspase-11 was required for inducing death of macrophages, while NLRP3 was dispensable, identifying caspase-11 has effects independent of the NLRP3caspase-1 axis. Taken together these results suggest caspase- 11 induces cell death and leads to restriction of Brucella infection.

Elevated lactate levels are observed in multiple forms of arthritis, including septic arthritis and rheumatoid arthritis (238). Patients with Brucella induced arthritis display similar synovial levels of lactate as patients with rheumatoid, or crystal-induced arthritis, but have higher lactate levels than osteoarthritis patients (239). Pyroptosis releases lactate dehydrogenase into the extracellular environment which can subsequently cause degradation of free L-lactate (240, 241). Alternatively, active caspase-1 can directly inhibit glycolysis and in turn Llactate by cleaving important glycolytic enzymes such as $\alpha$-enolase, aldolase and GAPDH (227). In an attempt to determine if inflammasomes restricted intracellular Brucella survival due to a low lactate environment, we inhibited glycolysis and subsequent lactate production using 2-DG in host macrophages. Treatment with 
2-DG reduced Brucella macrophage burdens; however, glycolysis inhibition also hindered IL-1 $\beta$ release, indicating 2-DG may impede inflammasome activity or IL$1 \beta$ mRNA expression. Glycolysis is important for NLRP3 inflammasome priming and has been shown to induce IL-1 $\beta$ in myeloid cells in response to LPS (242244). We demonstrated inhibiting glycolysis with 2-DG reduced swelling but did not alter joint Brucella loads in vivo. Further, experiments should determine if increasing doses of 2-DG or different glycolysis inhibitors could reduce Brucella burdens. Thus, glycolysis inhibitors could serve as potential therapeutics for Brucella-induced inflammation and infection due to their ability to inhibit excessive inflammasome-dependent inflammation while at the same time limiting lactate production and thus the survival of Brucella.

To address if inflammasomes restricted Brucella survival by reducing Llactate, without potentially altering inflammasome priming or activation, we made a Brucella mutant defective in L-lactate dehydrogenase. Similar to another study using B. abortus (101), we found that Brucella L-lactate dehydrogenase was important for intracellular B. melitensis survival. However, inflammasomes do not appear to aid in B. melitensis restriction through their ability to limit L-lactate, as the $\Delta / / d D$ complemented strain did not outcompete $\Delta / l d D$ to a greater degree in caspase-1/11-deficient cells relative to WT BMDMs. During acute or focal infection, there may be a requisite of L-lactate for Brucella survival in the host, suggesting host factors that induce L-lactate production may aid in Brucella's intracellular viability. Host metabolism is critical to Brucella infection, as the type of metabolism Brucella prefers may depend on the stage of infection $(100,101)$. One group 
demonstrated that in a model of chronic infection Brucella shifts host metabolism to fatty acid oxidation in order to utilize free host glucose (100). Thus, Brucella may prefer a glycolytic environment during acute or focal infection and beta-oxidation conditions during chronic infection.

Protection conferred by inflammasomes is due in part to IL-18. At day 7 post-infection restriction of Brucella burdens in the joint was partially dependent on IL-18, but independent of IL-1R signaling in the presence or absence of IL-18. Additionally, IL-1R was not responsible for protection during chronic joint infection (data not shown). Caspase-1/11 contributed to bacterial resistance to a greater extent than IL-18, indicating there are other inflammasome dependent mechanisms such as pyroptosis that contribute to Brucella restriction. IL-18 can induce production of IFN- $\gamma$, which is a well-known mediator of protection against Brucella infection and chronic Brucella-induced arthritis $(70,160)$. Some pathogen induced models have demonstrated IFN- $\gamma$ is secreted due to activation of inflammasomes and subsequent IL-18 production $(228,245)$. We found the bactericidal actions of caspase-1/11 and IFN- $\gamma$ were additive indicating the antimicrobial activity of caspase-1/11 is not completely dependent on inflammasome production of IFN- $\gamma$. Interestingly, the abrogation of IFN- $\gamma$ induced severe swelling and inflammation in WT mice starting at day 3 post-infection, and this inflammation was almost entirely dependent on caspase-1/11.

Prior to these studies, it was unclear if the protective effect of IFN- $\gamma$ against Brucella induced arthritis following intraperitoneal infection was due to restricting hematogenous spread of Brucella to the joint or if IFN- $\gamma$ acted locally to suppress 
arthritis $(70,165)$. Here we show IFN- $\gamma$ acts within the joint to reduce Brucella replication and inflammation, as footpad infected IFN- $\gamma$-deficient mice developed a more severe arthritis with increased joint Brucella burdens compared to WT mice. We previously demonstrated lymphocytes do not play a major role in the induction of arthritis in this model (160). Here we determined IFN- $\gamma$ neutralization enhances Brucella burdens in both Rag $1^{-/-}$mice and WT mice. This indicates there are cells independent of traditional lymphocytes that produce IFN- $\gamma$. Additionally, adaptive immune cell production of IFN- $\gamma$ is important for Brucella clearance, and NK cells or $\gamma \delta$ T-cells are not essential for protection against joint infection. Thus, the innate source of IFN- $\gamma$ remains unknown in our model. Tissue-resident type 1 innate lymphoid cells are known for their fast-acting production of IFN- $\gamma$ in response to pathogens and may be the protective IFN- $\gamma$ producing cell type $(246,247)$.

The ASC-dependent inflammasomes, AIM2 and NLRP3, are known to recognize Brucella and restrict $B$. abortus splenic burdens during chronic infection $(117,219)$. Surprisingly, AIM2 and NLRP3 were dispensable for control of joint infection. NLRP3 or AIM2 can compensate for one another as has been described in pulmonary aspergillosis, which could explain why we did not observe altered Brucella joint burdens in mice only lacking NLRP3 or AIM2 (248). Alternatively, caspase-1 may have an AIM2/NLRP3 independent activator specific to control of joint tissue. In addition to being dispensable for joint Brucella clearance, AIM2 did not induce joint swelling in the presence or absence of IFN-y. NLRP3 contributed modestly to joint swelling in the presence of IFN- $\gamma$, but only at day 2 post-infection. 
In contrast, NLRP3 played a major role in joint swelling in IFN-y neutralized mice throughout infection. Chronic human brucellosis patients develop an impaired IFN$\gamma$ response to Brucella compared to patients at the onset of disease (148). Thus, during chronic Brucella infection, attenuated IFN- $\gamma$ production may lead to enhanced NLRP3 activation and deleterious inflammation.

IFN- $\gamma$ can induce the NO producing enzyme iNOS. We found that iNOS expression in Brucella-infected mouse joints is IFN- $\gamma$-dependent. Although NO is generally thought to be inflammatory it can have immunosuppressive effects and is known to restrict Brucella replication in macrophages (143). Here we demonstrated iNOS deficiency increased arthritis and joint Brucella burden. Furthermore, iNOS deficiency enhanced production of IL-1 $\beta$ in the joint, possibly indicating increased inflammasome activation. NO can modulate signaling of kinases and transcription factors via thiol nitrosylation (s-nitrosylation), the addition of an NO group to a protein cysteine residue (249). NO can inhibit NLRP3 and caspase- 1 activation by s-nitrosylation of the proteins' active site $(231,250)$. This results in failed recruitment and activation of caspase-1, thus suppressing several key inflammatory processes $(161,230,250)$. The immunosuppressive effect of NO is protective in diseases with severe immunopathology such as LPS-induced septic shock (250), and in a murine model of tuberculosis (161). Similarly, IFNY/NO-dependent inhibition of inflammasome activation controls Brucella-induced inflammation, as we found that the NO donor, SNAP, inhibits caspase-1 activation in vitro, and that IFN- $\mathrm{y}$ is critical for controlling inflammasome-dependent pathology during Brucella infection in vivo. Unregulated inflammasome activation in the 
absence of IFN- $\gamma$ could also lead to mortality, as one study demonstrated T cell or IFN- $\gamma$ deficiency resulted in similarly elevated Brucella burdens in the spleen, lung and liver following intranasal infection; however, IFN- $\gamma$ deficient mice developed neutrophilia and succumbed to infection while T cell deficient mice do not (142). This suggests disease severity and mortality in IFN- $\gamma$ deficient mice is due to overwhelming pathology, possibly caused by an inability to inhibit inflammasome induced inflammation. Human brucellosis patients are more likely to have polymorphisms associated with reduced IFN-Y $(145,251)$; thus, impaired IFN-Y production in these patients could lead to deficient NO production and inflammasome-dependent pathology. NO donors are well tolerated in humans (252). Additionally, NO is antimicrobial against Brucella (143) and can also potentially inhibit deleterious caspase-1 and NLRP3-dependent inflammation (161). Thus, NO donors, in combination with antibiotics, might be potential therapeutic candidates to treat brucellosis, particularly in patients with impaired IFN-ץ production. 


\section{CHAPTER VI}

\section{Discussion}

Human brucellosis is a systemic disease of which arthritis is the most common focal complication $(56,57)$. Brucella antigen in the joints causes inflammation which initiates an often prolonged arthritis in humans (57). There are limited animal models for the study of human brucellosis as rodents do not recapitulate human symptomology and/or susceptibility. Thus, in vivo and tissue specific mechanisms of Brucella infection remain largely unexplored, despite a growing need for new therapeutics aimed at treating chronic arthritis. In these studies, we used a murine systemic infection model, and characterized a new model of Brucella-induced arthritis to understand the inflammatory responses induced by Brucella in the joints.

\section{Model Advantages and Disadvantages}

These studies utilized a previously described Brucella-induced arthritis model in which IFN- $\gamma$ deficient mice develop arthritis 2-4 weeks post systemic infection via inoculation by the intranasal, intraperitoneal or oral routes (165). We also defined and characterized a new model in which footpad infection of WT mice develop symptoms of arthritis as early as 1 day post-infection. While these models are the first reasonable murine systems for the study of Brucella-induced arthritis, both encompass several advantages and disadvantages. Humans generally 
develop arthritis through oral or aerosol transmission, thus the systemic model, but not the footpad model, recapitulates the traditional dissemination of bacteria to the joint $(16,17,165)$. In contrast, most humans have a sufficient IFN- $\gamma$ response, making the systemic IFN- $\gamma$-deficient model less biologically relevant $(165,253)$. It is also difficult to experimentally measure pertinent immune responses in mice that lack IFN- $\gamma$ signaling, as it is an important inducer of inflammation and bacterial clearance $(140,253,254)$. Inflammation generally, but not always, correlates with Brucella burdens in our studies. Mice containing genetic deletions may have increased dissemination of bacteria rather than a defect in the joint immune response, which can result in differential inflammation in the systemic model. Thus, in systemically infected mice it can be difficult to discern which immune responses may be mediated by local inflammation versus those resulting from differential dissemination. Footpad infected mice develop arthritis much earlier than intraperitoneal infected IFN- $\gamma$-deficient mice, indicating inflammation in this model is due to innate responses. When arthritis is evident in the systemic model, adaptive immune responses presumably have already been established, and mice succumb to infection around 6 weeks post-infection. Thus, these models may be advantageous depending on what responses are being measured (Table 4).

While these models present with various advantages and disadvantages experimental murine Brucella-induced arthritis is a valuable tool for studying immune responses. In both models swelling correlated with inflammation allowing us to conduct longitudinal studies in which swelling was measured as an indicator of the inflammatory response. Interesting time points could then be more 


\section{IFN- $\gamma$ deficient (systemic model)}

\section{Footpad (local model)}

- Biologically relevant route of joint infection (dissemination to the joint)

- Can study different routes of infection (e.g. oral, intranasal or intraperitoneal)
- WT mice (immune competent mice)

- Joint tissue specific immune responses

- Innate immune responses in the absence of adaptive priming

- Independent of bacterial dissemination

- Can study IFN-y responses

- Can study initiation and resolution of inflammation

- Can use murine infection models beyond 6 weeks post-infection

Table 4. Advantages of the murine models of Brucella-induced arthritis.

Comparisons of individual advantages of a systemic IFN- $\gamma$-deficient mouse model of Brucella-induced arthritis verse a local footpad model of Brucellainduced arthritis. 
thoroughly analyzed via necropsy of the animal and commencement of H\&E staining, CFU analysis, inflammatory cytokine quantification and other experimental assays. Similar to human arthritis both models exhibited joint inflammation independent of the Brucella species used to infect the animal (53-55, 165). With these new valuable models Brucella-induced arthritis, particularly footpad infected WT mice, are an invaluable system to not only study the immune response to Brucella, but to understand inflammatory processes within the joint.

\section{Inflammatory Dichotomy in the Joint Versus the Systemic Periphery}

Due to Brucella's immune evasion strategy and the many anti-inflammatory proteins it employs $(99,107,255)$, it is hypothesized that Brucella induces lowgrade inflammation which does not result in a large enough immune response to induce bacterial clearance. Thus, this low-grade inflammatory state is the presumed mechanism underlying bacterial persistence within the host (92). In contrast to this idea, we found that upon footpad infection tissues within the joint mount a strong and early inflammatory response to Brucella and then resolve inflammation. Similarly, brucellae begin to reach the joint at 2 weeks post-infection in systemically infected IFN- $\gamma$-deficient mice, concomitant with development of arthritis (165). Thus, we speculate that a robust inflammatory response to Brucella is specific to joint tissue and may be the reason for arthritic sequelae often associated with this disease. There may be truth to the dogma suggesting that a low grade inflammatory state induces bacterial persistence, as footpad infected mice inoculated with $1 \times 10^{6}$ bacteria resolve joint inflammation and induce joint 
Brucella clearance faster than mice infected with a dose of $1 \times 10^{3}$. We suspect efficient innate and adaptive immune priming requires a large amount of Brucella antigen and results in more rapid clearance and resolution of both the pathogen and inflammation. This may be a consequence of a stronger macrophage response during acute infection as inflammatory macrophages, specifically IFN- $\gamma$ activated macrophages contribute largely to Brucella clearance $(140,143)$. Therefore, footpad inoculation using large amounts of Brucella antigen and adjuvant may be an effective vaccine strategy as joint tissue induces an innate response that seems to prime adaptive immunity.

Previously it was found that following i.p. infection with $B$. melitensis, IL-1 contributes to clearance in the spleen of WT and IFN- $\gamma$ deficient mice at day 56 post-infection (165). In contrast, we did not observe a role for IL-1 in the clearance of bacteria from the joint or spleen following footpad infection of WT mice at day 30 post-infection. This indicates IL-1 may not be important for inducing Brucella clearance in joint tissues, or IL-1 only restricts of Brucella infection at timepoints later than 30 days post-infection. We suspect variation in the $\mathrm{IL}-1$ response between tissues could be due to the unique cellular subset, and how they respond to IL-1 present within the joint or periphery. Joints are composed of fibroblast-like synoviocytes (FLS), chondrocytes, osteocytes, immune cells and many other cell types (256). FLS are important for providing nutrients to the cartilage through their production of a lubricant called synovial fluid (257). FLS can also secrete a variety of pro-inflammatory cytokines during rheumatoid arthritis (258). Additionally, it is well known that synovial hyperplasia, or inability for FLS to undergo apoptosis, is 
a common manifestation of rheumatoid arthritis, and Brucella infection of FLS enhances host cell viability $(156,257,259,260)$. FLS hyperplasia, and subsequent increase in inflammatory cytokine production, can also be induced by IL-1 signaling (261). Since Brucella can infect FLS, proliferation of these cells may allow for an enhanced reservoir in the joint compared to the periphery (155). Future studies should investigate whether synoviocyte proliferation aids in Brucella survival within the joint or if their robust production of cytokines allows for Brucella clearance in joint tissues.

Prolonged inflammation is known to induce bone destruction through the differentiation of monocytes into osteoclasts. These specialized cells typically absorb bone during bone remodeling, a process known as osteoclastogenesis. Bone resorption is greater than formation during Brucella infection, thus causing erosion $(153,262,263)$. Inflammatory cytokines TNF- $\alpha$, IL-1 $\beta$ and IL-6 are known to induce bone resorption and are secreted upon FLS B. abortus infection, possibly contributing to the arthritic phenotype we observe $(187,264)$. Not much is known about chondrocytes during Brucella infection; however, they have been observed to secrete a variety of collagenases and proinflammatory cytokines such as IL-1 $\beta$ and TNF- $\alpha$ in osteoarthritis patients (265). Although these cells were not directly studied in our investigation, they may be important mediators of inflammation or pathology and aid in the understanding of how the pathways described here are related.

We found that activated IL-18 induces inflammation upon Brucella infection. Additionally, we showed CXCR2 but not CCR2 initiated Brucella-induced arthritis. 
These mechanisms may be joint specific as FLS can respond robustly to IL-18 which upregulates endothelial adhesion molecules and activates pro-inflammatory transcription factors (266). Additionally, human FLS from rheumatoid arthritis patients can produce the human CXCR2 ligand, CXCL8, which may result in enhanced neutrophil recruitment (267). IL-18 can also induce secretion of CXCR2 ligands but not CCR2 ligands in synovial fibroblasts isolated from rheumatoid arthritis patients (268). Thus, FLS may bridge the gap between inflammasome activated IL-18 and the recruitment of neutrophils via CXCR2 signaling. Alternatively, chondrocytes may inhibit synoviocyte IL-18 induced inflammation as they are strong producers of iNOS and subsequent NO production. NO manufactured by chondrocytes required IL-1 $\beta$ and/or TNF- $\alpha$ priming which we showed to be highly upregulated in Brucella infected joints (269).

\section{Innate and Adaptive IFN- $\gamma$ production}

Adaptive immune cells are partially responsible for arthritis resolution in IFN-Y sufficient mice, as Rag $1^{-1-}$ mice displayed increased inflammation at day 30 post footpad infection. This response was largely due to $\alpha \beta$ T-cells as mice lacking these cells exhibited defects in arthritis resolution, while mice deficient in B-cells did not express this phenotype (data not shown). Additionally, we demonstrated systemically infected IFN- $\gamma$-deficient mice do not resolve joint swelling. These data demonstrate T-cells mediate inflammation resolution in an IFN- $\gamma$ dependent manner. T-cells produce IFN- $\gamma$ especially when primed by IL-12 and IL-18 signaling (270). In WT footpad infected mice, we observed an increase in IFN- $\gamma$ producing 
CD8 and CD4 T-cells at 14 days post-infection compared to those at 8 days postinfection. Furthermore, footpad infected mice that lack adaptive immune cells displayed enhanced joint Brucella burdens compared to WT mice, and this protective effect was completely dependent on IFN- $\gamma$. Thus, T-cell induction of IFN$\gamma$ is needed for resolution of arthritis and Brucella joint clearance. IFN- $\gamma$ is also important for macrophage bactericidal activity and inflammatory macrophages play a key role in Brucella clearance $(140,143,271)$. Additionally, we did not, observe a difference in joint Brucella loads in WT IFN- $\gamma$-deficient mice compared to IFN- $\gamma$ neutralized mice which lacked the monocyte recruitment receptor CCR2. Thus, we predict IFN- $\gamma$ is needed for macrophage activation and in turn clearance of Brucella in the joint. Collectively we believe T-cell IFN- $\gamma$ production and in turn macrophage activation are vital for joint Brucella clearance and arthritis resolution.

Activation of T-cells and their subsequent IFN- $\gamma$ production generally has an onset of about 1-2 weeks (272). Footpad infected IFN- $\gamma$ deficient mice however, develop robust inflammation, compared to infected WT-IgG mice, as early as 3 days post-infection. Furthermore, systemically infected Rag $1^{-/-}$mice neutralized of IFN- $\gamma$ displayed reduced swelling compared to WT anti-IFN- $\gamma$ treated mice, but they still exhibited a robust amount of swelling compared to WT-IgG treated mice. Additionally, footpad infected WT mice depleted of NK cells or $\gamma \delta$ T-cells did not display an increase in their inflammatory phenotype or Brucella joint clearance compared to WT animals. This indicates that innate IFN- $\gamma$ also plays an important role in the clearance of Brucella from the joint, and that an unconventional cell type may induce IFN- $\gamma$. Systemic infection of the spleen begins to resolve inflammation 
and clear Brucella at roughly 2 weeks post-infection, which follows the timeline of adaptive IFN- $\gamma$ responses $(165,272)$. In contrast, Brucella clearance and swelling resolution are both generally evident by day 3 post-footpad infection, and leukocyte recruitment begins to dampen around day 7 . Thus, this timely IFN- $\gamma$ response appears to be native to the joint. ILCs may induce this early IFN- $\gamma$ response however this does not explain the dichotomy of clearance in the joints versus the spleen as ILCs are not cells solely native to the joint $(247,273)$. Thus, specific joint resident cells such as FLS may also be vital sources of IFN- $\gamma$ as cultured FLS spontaneously make IFN- $\gamma$ and it is upregulated in patients with rheumatoid arthritis (257).

\section{Inflammation v. Bacterial Clearance}

Dogma suggests there is often a tradeoff for inflammation. While inflammation works to clear the pathogen, it also induces pathology (76). Furthermore, Brucella preferentially replicates in immune cells such as macrophages further confounding this paradox, as macrophages can control Brucella burden (143). We found that deletion of CXCR2 reduced inflammation but did not increase bacterial loads. This may indicate that there is "counterproductive inflammation", or inflammation that contributes to pathology but not Brucella clearance. However, these experiments were conducted in an IFN- $\gamma$-deficient murine model. Thus, repeat studies in footpad infected WT and CXCR2 ${ }^{-/-}$mice could demonstrate if this inflammation is truly "counterproductive" or if CXCR2 signaling requires IFN- $\gamma$ to restrict Brucella burdens. Unlike CXCR2 signaling, 
MyD88, IL-18, caspase-1, and caspase-11 initiate early inflammation ( day 2) which appears to reduce Brucella loads at later time points ( day 7). MyD88 ${ }^{-/}$, IL$18^{-l_{-}}$, caspase $1 / 11^{--}$and caspase $-11^{-l_{-}}$mice all exhibited delayed inflammatory responses, as all developed increased swelling between days 3-7 post-infection. Delayed inflammation could be a result of increased recognition of Brucella PAMPs, but is more likely due to increased DAMPs induced by overwhelming Brucella infection. DAMPs can be powerful immune stimulants and are found in large abundance in the synovial tissue of patients with rheumatic disease (206). We found that pyroptosis was driven by Brucella infection, particularly Brucella LPS. This lytic form of cell death is known to release DAMPs (206). High mobility group box 1 (HMGB1) is a common DAMP that is released during an LPSmediated endotoxin shock model. HMGB1 induced inflammation in LPS treated mice in an inflammasome dependent manner, but inflammation was independent of IL-18 and IL-1 $\beta$ signaling (274). Thus, overwhelming CFUs and inflammasome activation may induce DAMPS which can lead to the inflammatory phenotype seen in Brucella-induced arthritis.

\section{Neutrophils are Mediators of Brucella-induced Inflammation}

Neutrophils were clearly one of the primary cell types recruited to the joint upon Brucella-induced inflammation. We demonstrated this recruitment was in large part CXCR2 dependent and CXCR2 ligands, CXCL1 and CXCL2 were substantially upregulated in infected mouse joints. Similarly, the human CXCR2 ligand, CXCL8, is elevated in humans with Brucella induced arthritis (66). 
Neutrophil recruitment correlated with severity of arthritis. For instance, we observed enhanced neutrophil recruitment in the joints of footpad infected antiIFN- $\gamma$ treated mice compared to WT mice. Similarly, we observed a reduction in neutrophils in MyD88 ${ }^{-/-}$mice compared to WT at day 2 post Brucella footpad infection. Thus, depleting neutrophils, neutrophil recruitment or CXCR2 ligands may work as a sufficient therapy for treating excessive inflammation in the joint.

\section{Possible Mechanisms of MyD88 Dependent Inflammation}

MyD88 has long been known to protect mice from Brucella infection (203, 204); however, it remains controversial as to what initial receptors, or TLRs, are contributing to MyD88 activation as Brucella generally has weak immune recognition in the systemic periphery. Our footpad model exhibits robust MyD88dependent "productive inflammation." Thus, it is an excellent candidate for studying Brucella activation of TLRs, and understanding their role in inflammation and bacterial clearance. Additionally, DAMPs released during pyroptosis, such as HMGB1, may also signal through MyD88 via HMGB1 initiation of TLR4 and TLR2 signaling $(275,276)$. TLR4 signaling can also signal through TRIF allowing inflammatory signal transduction to occur even in the absence of MyD88 (105). Although one study revealed TRIF does not contribute to clearance of Brucella in the spleen and peritoneal cavity following i.p. Brucella infection (205), TRIF signaling has not been evaluated in the joints. Hence, this signaling event could lead to the inflammation seen in MyD88 $8^{-/-}$mice around day 7 post-infection. 
MyD88 is essential for priming many inflammatory components including inflammasome proteins, (277) pro-IL-1 $\beta$, pro-IL-18 and other inflammatory cytokines and chemokines such as TNF- $\alpha$ and CXCR2 ligands $(117,278)$. We have demonstrated that MyD88 is critical for IL-1 $\beta$ production in the joint. Similarly, others have described MyD88 is required for caspase-1 activation in Brucella infected BMDMs (117). Considering MyD88's extensive role in regulating a multitude of inflammatory responses, MyD88 is likely required for some inflammasome dependent inflammation in Brucella induced arthritis. MyD88 mediated inflammation is "productive" and works to clear bacteria in the joint. This may be due to MyD88/inflammasome dependent IL-18, as IL-18 can encourage IFN- $\gamma$ production (279). However, MyD88 has protective effects beyond IFN- $\gamma$ as i.p. infected MyD88 ${ }^{-/}$-anti-IFN- $\gamma$ treated mice succumb to infection sooner than WT-anti-IFN- $\gamma$ treated animals (data not shown). Additionally, MyD88 ${ }^{-/-}$mice were defective in resolution of inflammation and bacterial clearance (day 28), which was similar to observations in mice lacking adaptive immune cells (Rag $1^{-/-}$mice). MyD88 $^{-/-}$mice also exhibited reduced IFN- $\gamma$ production by CD4 and CD8 T-cells, and displayed decreased anti-Brucella antibodies in sera compared to WT mice (data not shown). This indicates MyD88 is at least partially responsible for priming the adaptive immune response to Brucella. We suspect that MyD88 dependent IL18R signaling in T cells is important for clearance of Brucella at later time points. In particular, we hypothesize T cells produce IFN- $\gamma$ in response to IL-18 that aids in macrophage activation $(140,279,280)$. 


\section{Metabolism's Role During Brucella Infection}

Although we believe quick, robust, macrophage activation and, in turn, MyD88 signaling is important for clearing Brucella, MyD88 activation after the initial state of recognition may be a disadvantage to the host in certain cell types. Bacterial LPS can induce TLR-signaling, and presumably MyD88 signaling in macrophages, shifting their metabolism to a glycolytic state. (281). We and others have demonstrated that the byproduct of glycolysis, lactate, aids in Brucella survival (101), and preliminary studies indicate infected MyD88 ${ }^{-/-}$BMDMs do not produce lactate to the same magnitude as infected WT cells (data not shown). Although MyD88 ${ }^{-/}$mice exhibit defects in Brucella clearance, MyD88 signaling in infected cells may be intrinsically detrimental to the host. We suggest MyD88 dependent cytokines are important for restricting Brucella, but MyD88 intrinsic induction of lactate aids Brucella in establishing its replicative niche. Indeed, we found that mice lacking MyD88 in myeloid cells make reduced TNF- $\alpha$, which we found to be protective against Brucella infection, however MyD88-signaling in myeloid cells does not aid in Brucella clearance (data not shown). MyD88's production of lactate may contribute to Brucella's preference of macrophages as their favored host. While Brucella replicates in alternatively activated macrophages which utilize fatty acid $\beta$-oxidation as their primary metabolism (100, 242), it is unclear if Brucella prefers glycolytic cells over $\beta$-oxidative cells during acute infection or if increased lactate aids Brucella in establishing its intracellular niche. Future studies should elucidate the role of metabolism in acute versus 
chronic infection and determine if MyD88 signaling, and in turn glycolytic metabolism, aid in Brucella replication in specific cell types such as macrophages.

Enhanced MyD88-dependent glycolysis can also lead to the production of the pro-inflammatory cytokine IL-1 $\beta(103,242)$. We demonstrated the glycolysis inhibitor 2-dG restricts IL-1 $\beta$ secretion and intracellular Brucella burdens in macrophages. This may be due to reduced production of MyD88 dependent proIL-1 $\beta$ (242) or MyD88-dependent inflammasome activation/priming $(117,277)$, as glycolysis has been shown to induce inflammasome proteins such as NLRP3 (243, 244). Additionally, monocytes from rheumatoid arthritis patients produce increased NLRP3 dependent IL-1 $\beta$ when given increasing doses of glucose (282). When we inhibited glycolysis with 2-dG in the footpad model of Brucella induced arthritis, mice exhibited reduced joint swelling without altering joint Brucella burdens. 2-dG treated BMDMs also displayed decreased levels of IL-1 $\beta$, indicating glycolysis is critical for IL-1 $\beta$ priming and/or inflammasome priming/activation. Further studies need to investigate optimal dosage of 2-dG and if increasing the concentration of 2-dG can enhance or reduce Brucella clearance. Mechanisms for glycolysis dependent inflammation should additionally be studied in Brucella induced arthritis in order to determine the critical cell types involved in pathology and if glycolysis is truly MyD88-dependent in our model. Others have observed glucose metabolism in fibroblast-like synoviocytes can contribute to human rheumatoid arthritis (283). Taking these phenotypes into account 2-dG may be a complementary therapy for reducing Brucella induced arthritis. 


\section{IFN- $\gamma$ Dependent Mechanisms for Controlling Inflammasome Dependent Inflammation}

Inflammasomes contributed extensively to Brucella-induced arthritis particularly in the absence of IFN- $\gamma$. We demonstrated in vitro that NO inhibits caspase-1 activation, but it is unknown how and where in the inflammasome pathway NO is suppressive. Similar to other studies of inflammasome mediated pathology, further experiments should investigate if NO s-nitrosylates NLRP3 and/or caspase-1 $(161,231,250)$. Additionally, to our knowledge no studies have determined if the caspase-11 pathway can be suppressed by NO via snitrosylation. Thus, future investigations should also determine if NO donors such as SNAP can limit pathology in the joint or even in the systemic periphery of IFN$\gamma$-deficient mice. Intraperitoneal infected IFN- $\gamma$-deficient mice succumb to infection while T-cell deficient mice survive (142). Death in these mice is due to overwhelming neutrophilia, as both types of immunocompetent mice had similarly elevated bacterial loads. We suspect this phenotype is due to inflammasome activation and $\mathrm{IL}-18$ release which can result in increased recruitment of neutrophils (284).

In vivo experiments demonstrated $\mathrm{NOS}^{-/-}$mice developed more robust swelling compared to WT mice, but this phenotype was not as severe as that seen IFN- $\gamma$ neutralized mice. Additionally, IFN- $\gamma$ deficient mice had enhanced joint Brucella loads compared to WT and $\mathrm{NOS}^{-/-}$mice. Thus, IFN- $\gamma$ may also prevent inflammation by reducing Brucella loads and/or IFN- $\gamma$ may have other roles in limiting inflammasome-induced inflammation. A regulatory role of IFN- $\gamma$ has been observed in a murine rheumatoid arthritis model; however, suppression of 
inflammation was only speculated to be due to IFN- $\gamma$ inhibition of inflammasomes as IFN- $\gamma \mathrm{R}^{-/-}$mice had reduced levels of activated caspase-1 compared to WT (285). Preliminary experiments show caspase-11 is primed and activated to a greater degree in IFN- $\gamma$-deficient mouse joints (data not shown). We demonstrated caspase-11 contributes to arthritis in the absence of IFN- $\gamma$, indicating inflammation inhibited by NO could potentially be due to caspase-11. Macrophages in vitro require IFN- $\gamma$ or type I IFNs for caspase-11 priming (286). Hence, enhanced priming of capspase-11 may be due to type I IFNs and should be further investigated. ER stress can also induce priming of caspase-11 which has been shown to contribute to LPS induced pathology (287). Brucella is considered a model organism for activating host ER stress (288). Therefore, ER stress may lead to enhanced caspase-11 priming and in turn inflammation.

\section{Mechanism of IL-18 induced Inflammation}

We demonstrated inflammasome activation of IL-18 contributes to Brucellainduced arthritis development, but the mode in which IL-18 mediates inflammation is currently unknown in our model. IL-18 can activate neutrophils as human peripheral blood derived neutrophils constitutively express IL-18R. Neutrophils from rheumatoid arthritis patients respond to IL-18 by inducing enhanced production of inflammatory cytokine and chemokines. IL-18 activated neutrophils display an increase in granule release, increase respiratory bursts and secreted cytokine/chemokine including TNF- $\alpha$ and CXCL8 (284). Additionally, neutrophils are recruited to the peritoneal cavity upon i.p. administration of IL-18 in WT mice. 
Similarly, treatment with IL-18 receptor antagonist (IL-18RA) reduced swelling and concentrations of TNF- $\alpha$ and myeloperoxidase in the joints of a mouse model of paw edema (284). IL-18 also contributes to a model of streptococcal cell wall induced arthritis in an IFN- $\gamma$ independent manner (289). These studies along with our own underscore the possibility that IL-18R along with CXCR2 initiate the pathogenic recruitment of neutrophils in murine Brucella-induced arthritis. Moreover, CXCR2 antagonists in combination with IL-18RA may reduce pathology in human patients.

\section{Cell Death}

It is unclear what type of cell death is best for facilitating Brucella clearance. We demonstrated that Brucella LPS-induced cell death is mediated through pyroptosis. Additionally, caspase-1 and caspase-11 BMDMs contain less intracellular bacteria which was independent of NLRP3 or AIM2 signaling. We hypothesize caspase- 1 and caspase-11 clear bacteria by eliminating its macrophage reservoir, but pyroptosis has also been demonstrated to clear bacteria by trapping them in PITs then promoting neutrophil efferocytosis of the pyroptotic cell $(215,290)$. Brucella can resist bactericidal killing inside the neutrophil environment $(207,291)$; however the process of undergoing pyroptosis also damages the bacteria making them more susceptible to microbial killing by ROS and antimicrobial peptides $(215,290)$. Although some intracellular pathogens are cleared by apoptosis (292), Brucella canonically inhibits cell death to promote its replication (154). Brucella LPS can also dissociate from smooth to rough in 
order to encourage cell death, allowing release of the bacteria and further dissemination $(126,293)$. Considering pyroptosis eliminates the bacterial reservoir while possibly damaging the bacteria leading to effective neutrophil clearance, pyroptosis may be more advantageous to the host than apoptosis or other forms of cell death.

\section{Guanylate Binding Proteins as Inducers of Inflammasome Activation}

Recently it was shown that type I IFN dependent GBPs are important for canonical inflammasome recognition, and consequential caspase- 1 activation and clearance of Brucella (118). GBP have also been described as essential for noncanonical inflammasome activation, as GBPs can expose LPS contained inside the pathogen containing vacuole to the cytosol. This exposure allows LPS to be recognized by caspase-11 (294). Other studies suggest GBPs can act as an LPS adaptor which recognizes LPS and permits caspase-11 activation $(295,296)$. Although we have demonstrated IFNAR partially limits swelling at day 3 post footpad infection (data not shown), the role of type 1 IFNs and GBPs in our model is not clear. We found that Brucella's non-canonical hepta-acylated LPS activates caspase-11, but it is unknown if GBPs are required as adaptors for caspase-11 recognition and/or needed for LPS exposure to the cytosol. Future studies should identify if GBP, or caspase-11 in general, can recognize hepta-acylated LPS and other non-canonical bacterial LPS structures. Investigations should also analyze whether GBPs are needed for inflammasome induced inflammation/clearance and 
whether GBPs directly recognize LPS and/or release Brucella antigen from the BCV.

\section{Potential Therapeutics for the Treatment of Brucella-Induced Arthritis}

In these investigations, we identified several candidate targets for the therapeutic treatment of Brucella-induced arthritis. The most favorable therapeutics are likely those inhibiting inflammatory mediators that lead to "counter productive inflammation", but do not impact clearance of Brucella. Although extensive study of these potential treatments need to be investigated in several organ systems of WT mice, our most favorable candidates are CXCR2 antagonists, 2-dG and potentially NO donors. CXCR2 antagonists such as reparixin have been shown to reduce antigen induced arthritis in mice (192). CXCR2 antagonists have also been used in humans to decrease the growth of tumors and to treat pulmonary disease $(297,298)$. As numerous models of disease suggest a pathological role for CXCR2, extensive studies have been dedicated to safe and effective development of CXCR2 antagonists (190, 299-301). Additionally, 2-DG inhibits human monocyte chemotaxis as glycolysis can aid in myeloid cell mediated inflammation $(302,303)$. Similarly, studies have demonstrated 2-dG treatment can suppress neutrophil activity and 2-dG therapy is well tolerated in humans $(304,305)$. NO donors may also be effective therapies, as we demonstrated NO can inhibit inflammasome mediated inflammation and can be bactericidal against Brucella $(143,306)$. Further investigation on dosing and types of NO donors warrant deeper investigation. In addition, IL-1 was identified 
as a contributor of joint inflammation. Although IL-1 did not confer Brucella clearance in the joint, it was protective in the spleen of WT mice. Thus, it may be more advantageous to design an IL-1 therapy that would remain locally within the joint. Extensive research is working to advance intraarticular injections and novel drug delivery systems to keep drugs contained within the joints in order to combat articular inflammatory diseases $(307,308)$.

Mediators that induced inflammation but also contribute to clearance of Brucella may likewise be useful therapy targets, as all treatments described here are presumed to be used in combination with antibiotics. Inflammasome inhibitors for the treatment of several inflammatory diseases have long been suggested and several small molecule inflammasome inhibitors exist $(309,310)$. To our knowledge, no human clinical trials have investigated the effects of specific inflammasome inhibitors on inflammatory disease. However, Colchicine, a recognized anti-inflammatory agent, was demonstrated to reduce IL-1 $1 \beta$, IL-18 and pro-caspase-1 mRNA and active caspase-1 in humans with acute coronary syndrome (311). MyD88 suppression may also be an effective therapy; however, due to MyD88's central role in inflammation and subsequent bacterial clearance, it may be our least likely therapeutic candidate. Further studies should differentiate the "productive" versus "counterproductive" inflammatory role downstream or upstream (TLRs) of MyD88 in order to further hone in on the deleterious inflammation initiating pathology. 


\section{Conclusion}

This work represents substantial progress in understanding how Brucella activates the immune system and induces pathology. We demonstrated CXCR2 dependent neutrophil recruitment was largely pathogenic in the joints following i.p. Brucella infection in mice threated with anti-IFN- $\gamma$. Understanding the possible pitfalls of using IFN- $\gamma$-deficient mice we generated a new model of Brucella induced arthritis, giving researchers a valuable tool for understanding pathology in the joint following Brucella footpad infection. Pervious Brucella work has focused almost entirely on systemic responses which have weak innate immune recognition of Brucella. This new model has allowed us to investigate how joint specific tissues respond to infection independent of bacterial dissemination to the joint. This model also allows for longitudinal studies in mice that induce quick, robust, immune responses which we believe to be specific to joint tissue. We determined these vigorous immune responses initially rely on MyD88 signaling, which is important for subsequent clearance of Brucella and resolution of inflammation. This inflammatory state at least partly relies on glycolytic metabolism to initiate such responses. Finally, we demonstrated inflammasomes confer inflammation in an IL-18 and IL-1 dependent manner, and inflammasome-dependent IL-18 and pyroptosis restrict Brucella infection. Pyroptosis was shown to be caspase-11 dependent, a mechanism previously thought to be evaded by Brucella (122). IFN$\gamma$ is protective against Brucella-induced pathology partly due to its production of NO, which was shown to suppress inflammasome mediated inflammation. The inflammatory pathways described in this work are likely interconnected, are there 
are likely many others that are currently unknown (Fig 35). Further research should consider what cells, particularly those specific to the joint, recognize Brucella and initiate robust articular inflammatory responses. Studies may also examine if MyD88 dependent glycolytic cell metabolism is advantageous to the host or the pathogen during acute Brucella infection. Finally, investigations should examine other pathways in which IFN- $\gamma$ suppresses inflammasome mediated inflammation. 


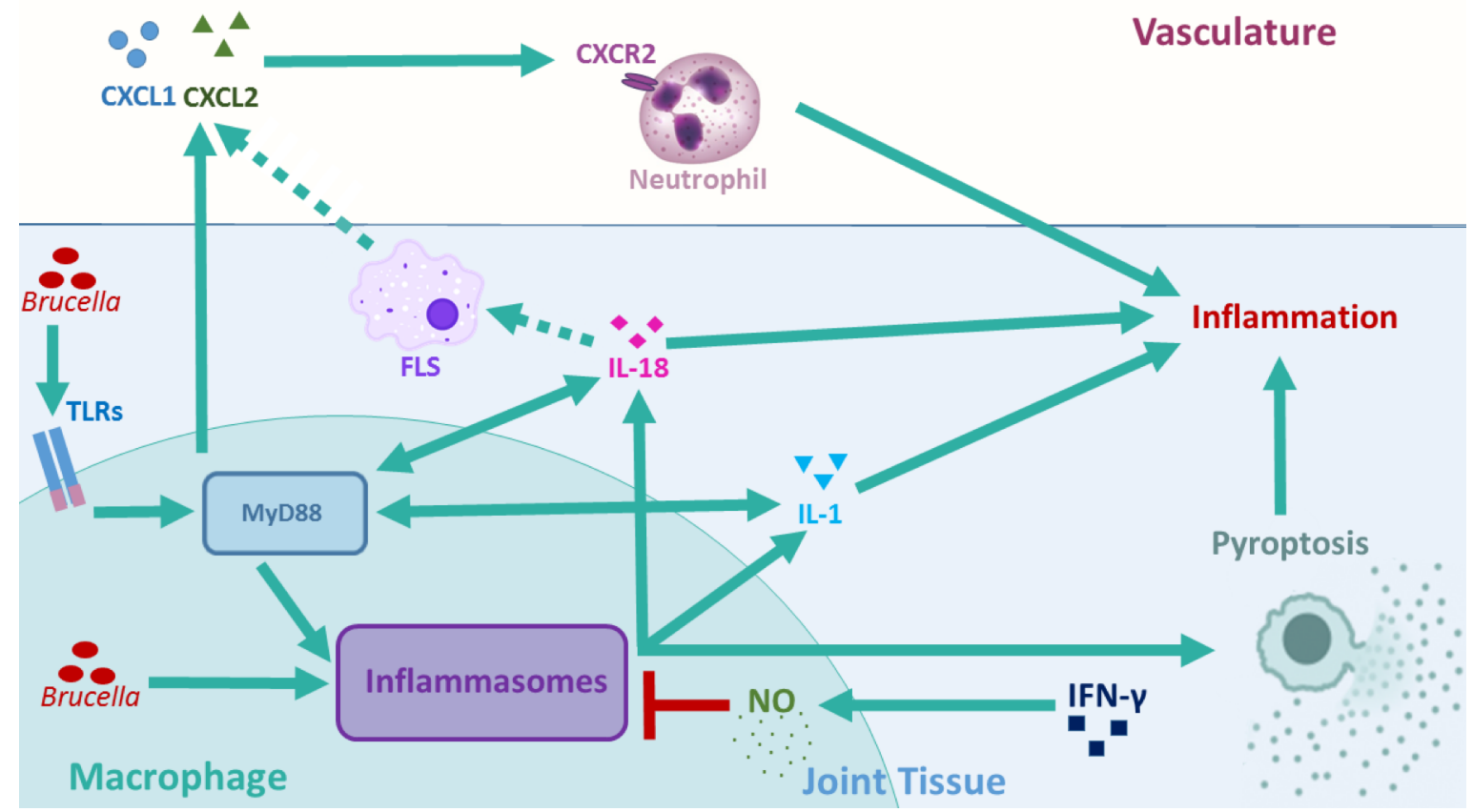

Figure 35. Possible mechanisms of inflammation during Brucella induced arthritis.

Brucella can be recognized by TLR signaling via MyD88 and inflammasomes. MyD88 can prime inflammasome components, IL-18, IL-1, CXCL1 and CXCL2. CXCL1 and CXCL2 signal through the neutrophil receptor CXCR2, then neutrophils are recruited to the joint tissue. Inflammasomes are required for IL-1 and IL-18 activation and secretion which all can enhance joint inflammation. IL-1 and IL-18 can signal through their respective receptors downstream to MyD88. IL18 may signal through FLS which can induce the production of CXCL1 and CXCL2. Inflammasomes can induce pyroptosis which may also lead to enhanced joint inflammation. Finally, IFN-y-dependent nitric oxide can inhibit inflammasome activation therefore inhibiting joint inflammation. Arrows represent pathways confirmed by us and others during Brucella infection, while dashed arrows indicate our hypothesis. $\vdash$ Indicates inhibition of a pathway. 


\section{References}

1. N Xavier M, A Paixao T, B den Hartigh A, M Tsolis R, L Santos R. 2010. Pathogenesis of Brucella spp. The Open Vet Sci J 4.

2. Roth F, Zinsstag J, Orkhon D, Chimed-Ochir G, Hutton G, Cosivi O, Carrin G, Otte J. 2003. Human health benefits from livestock vaccination for brucellosis: case study. Bull World Health Organ. 81:867-876.

3. Roop RM, Gaines JM, Anderson ES, Caswell CC, Martin DW. 2009. Survival of the fittest: how Brucella strains adapt to their intracellular niche in the host. Med Microbiol Immunol. 198:221-238.

4. Franco MP, Mulder M, Gilman RH, Smits HL. 2007. Human brucellosis. The Lancet Infect Dis. 7:775-786.

5. Young EJ. 1983. Human brucellosis. Review of Infectious Diseases 5:821-842.

6. Çelebi G, Külah C, Kiliç S, Üstündağ G. 2007. Asymptomatic Brucella bacteraemia and isolation of Brucella melitensis biovar 3 from human breast milk. Scand Jof Infect Dis. 39:205-208.

7. Young EJ. 1995. An overview of human brucellosis. Clin Infect Dis. 21:283-289.

8. Pappas G, Papadimitriou P, Akritidis N, Christou L, Tsianos EV. 2006. The new global map of human brucellosis. Lancet Infect Dis. 6:91-99.

9. Chomel BB, DeBess EE, Mangiamele DM, Reilly KF, Farver TB, Sun RK, Barrett LR. 1994. Changing trends in the epidemiology of human brucellosis in California from 1973 to 1992: a shift toward foodborne transmission. J Infect Dis. 170:1216-1223.

10. World Health Organization. 2012. Research priorities for zoonoses and marginalized infections. World Health Organ Tech Rep Ser:ix-xi, 1-119, $112 \mathrm{p}$ following 119.

11. Silva T, Costa EA, Paixão TA, Tsolis RM, Santos RL. 2011. Laboratory animal models for brucellosis research. J Biomed Biotechnol. 2011:518323.

12. Doganay M, Aygen B. 2003. Human brucellosis: an overview. Int J Infect Dis. 7:173-182.

13. Halling SM, Peterson-Burch BD, Bricker BJ, Zuerner RL, Qing Z, Li L-L, Kapur V, Alt DP, Olsen SC. 2005. Completion of the genome sequence of Brucella 
abortus and comparison to the highly similar genomes of Brucella melitensis and Brucella suis. J Bacteriol. 187:2715-2726.

14. Boschiroli M-L, Foulongne V, O'Callaghan D. 2001. Brucellosis: a worldwide zoonosis. Curr Opin Microbiol. 4:58-64.

15. Barquero-Calvo E, Conde-Alvarez R, Chacón-Díaz C, Quesada-Lobo L, Martirosyan A, Guzmán-Verri C, Iriarte M, Mancek-Keber M, Jerala R, Gorvel JP. 2009. The differential interaction of Brucella and Ochrobactrum with innate immunity reveals traits related to the evolution of stealthy pathogens. PloS one 4:e5893.

16. Zinsstag J, Roth F, Orkhon D, Chimed-Ochir G, Nansalmaa M, Kolar J, Vounatsou P. 2005. A model of animal-human brucellosis transmission in Mongolia. Prev Vet Med. 69:77-95.

17. Kaufmann AF, Fox MD, Boyce JM, Anderson DC, Potter ME, Martone WJ, Patton CM. 1980. Airborne spread of brucellosis. Ann N Y Acad Sci. 353:105-114.

18. Abo-Shehada MN, Odeh JS, Abu-Essud M, Abuharfeil N. 1996. Seroprevalence of brucellosis among high risk people in northern Jordan. Int $\mathrm{J}$ Epidemiol. 25:450-454.

19. Ruben B, Band J, Wong P, Colville J. 1991. Person-to-person transmission of Brucella melitensis. Lancet. 337:14-15.

20. Tuon FF, Gondolfo RB, Cerchiari N. 2017. Human-to-human transmission of Brucella - a systematic review. Trop Med Int Health. 22:539-546.

21. Weinstein RA, Singh K. 2009. Laboratory-acquired infections. Clin Infect Dis. 49:142-147.

22. Greenfield RA, Drevets DA, Machado LJ, Voskuhl GW, Cornea P, Bronze MS. 2002. Bacterial pathogens as biological weapons and agents of bioterrorism. Am J Med Sci. 323:299-315.

23. Pappas G, Panagopoulou P, Christou L, Akritidis N. 2006. Biological weapons. Cell Mol Life Sci. 63:2229-2236.

24. Rotz LD, Khan AS, Lillibridge SR, Ostroff SM, Hughes JM. 2002. Public health assessment of potential biological terrorism agents. Emerg Infect Dis. 8:225.

25. Ariza J, Corredoira J, Pallares R, Viladrich PF, Rufi G, Pujol M, Gudiol F. 1995. Characteristics of and risk factors for relapse of brucellosis in humans. Clin Infect Dis. 20:1241-1249. 
26. Ariza J, Gudiol F, Pallares R, Viladrich PF, Rufi G, Corredoira J, Miravitlles MR. 1992. Treatment of human brucellosis with doxycycline plus rifampin or doxycycline plus streptomycin: a randomized, double-blind study. Ann Intern Med. 117:25-30.

27. Ariza J, Bosilkovski M, Cascio A, Colmenero JD, Corbel MJ, Falagas ME, Memish ZA, Roushan MRH, Rubinstein E, Sipsas NV. 2007. Perspectives for the treatment of brucellosis in the 21st century: the loannina recommendations. PLoS Med. 4:e317.

28. Castaño MJ, Solera J. 2009. Chronic brucellosis and persistence of Brucella melitensis DNA. J Clin Microbiol. 47:2084-2089.

29. Khateeb MI, Araj GF, Majeed SA, Lulu A. 1990. Brucella arthritis: a study of 96 cases in Kuwait. Annals of the rheumatic diseases 49:994-998.

30. Foster JT, Beckstrom-Sternberg SM, Pearson T, Beckstrom-Sternberg JS, Chain PS, Roberto FF, Hnath J, Brettin T, Keim P. 2009. Whole-genome-based phylogeny and divergence of the genus Brucella. J Bacteriol. 191:2864-2870.

31. Sohn AH, Probert WS, Glaser CA, Gupta N, Bollen AW, Wong JD, Grace EM, McDonald WC. 2003. Human neurobrucellosis with intracerebral granuloma caused by a marine mammal Brucella spp. Emerg Infect Dis. 9:485.

32. Hernández-Mora G, Palacios-Alfaro J, Gonzalez-Barrientos R. 2013. Wildlife reservoirs of brucellosis: Brucella in aquatic environments. Rev Sci Tech. 32:89103.

33. Minas A, Minas M, Stournara A, Tselepidis S. 2004. The "effects" of Rev-1 vaccination of sheep and goats on human brucellosis in Greece. Prev Vet Med. 64:41-47.

34. Wallach J, Ferrero M, Delpino MV, Fossati C, Baldi P. 2008. Occupational infection due to Brucella abortus S19 among workers involved in vaccine production in Argentina. Clin Microbiol Infect. 14:805-807.

35. Ashford DA, Di Pietra J, Lingappa J, Woods C, Noll H, Neville B, Weyant R, Bragg SL, Spiegel RA, Tappero J. 2004. Adverse events in humans associated with accidental exposure to the livestock brucellosis vaccine RB51. Vaccine 22:3435-3439.

36. Corbel MJ. 1997. Brucellosis: an overview. Emerg Infect Dis. 3:213-221.

37. Davis D, Elzer P. 2002. Brucella vaccines in wildlife. Vet Microbiol. 90:533-544.

38. Godfroid J. 2002. Brucellosis in wildlife. Revue Scientifique et Technique-Office international des épizooties. 21:277-286. 
39. Nielsen K, Duncan JR. (ed). 1990. Animal brucellosis. CRC press. Boca Raton, Florida.

40. Corbel MJ. 2006. Brucellosis in humans and animals. World Health Organization. http://www.who.int/csr/resources/publications/Brucellosis.pdf.

41. Wanke MM. 2004. Canine brucellosis. Anim Reprod Sci. 82-83:195-207.

42. Mableson HE, Okello A, Picozzi K, Welburn SC. 2014. Neglected zoonotic diseases-the long and winding road to advocacy. PLoS Negl Trop Dis. 8:e2800.

43. Shen MW. 2008. Diagnostic and therapeutic challenges of childhood brucellosis in a nonendemic country. Pediatrics. 121:e1178-e1183.

44. Byndloss MX, Tsolis RM. 2016. Brucella spp. virulence factors and immunity. Annu Rev Anim Biosci. 4:111-127.

45. Gul S, Khan A. 2007. Epidemiology and epizootology of brucellosis: A review. Pak Vet J. 27:145.

46. Avila-Calderón ED, Lopez-Merino A, Sriranganathan N, Boyle SM, ContrerasRodríguez A. 2013. A history of the development of Brucella vaccines. BioMed Res Int. 2013:743509.

47. McDermott J, Grace D, Zinsstag J. 2013. Economics of brucellosis impact and control in low-income countries. Rev Sci Tech. 32:249-261.

48. Luna-Martínez JE, Mejía-Terán C. 2002. Brucellosis in Mexico: current status and trends. Vet Microbiol. 90:19-30.

49. Seleem MN, Boyle SM, Sriranganathan N. 2010. Brucellosis: a re-emerging zoonosis. Vet Microbiol.140:392-398.

50. Franc K, Krecek R, Häsler B, Arenas-Gamboa A. 2018. Brucellosis remains a neglected disease in the developing world: a call for interdisciplinary action. BMC public health. 18:125.

51. Costard S, Espejo L, Groenendaal H, Zagmutt FJ. 2017. Outbreak-related disease burden associated with consumption of unpasteurized cow's milk and cheese, United States, 2009-2014. Emerg Infect Dis. 23:957-964.

52. Angulo FJ, LeJeune JT, Rajala-Schultz PJ. 2009. Unpasteurized milk: a continued public health threat. Clin Infect Dis. 48:93-100.

53. Coventry MB, Ivins JC, Nichols DR, Weed LA. 1949. Infection of the hip by Brucella suis. JAMA. 141:320-325. 
54. Jaffray D, MacKenzie IG. 1979. Brucella abortus arthritis. Arthritis Rheum. 22:806.

55. Solera J, Lozano E, Martínez-Alfaro E, Espinosa A, Castillejos ML, Abad L. 1999. Brucellar spondylitis: Review of 35 cases and literature survey. Clinl Infect Dis. 29:1440-1449.

56. Rajapakse CN. 1995. Bacterial infections: osteoarticular brucellosis. Baillieres Clin Rheumatol. 9:161-177.

57. Gotuzzo E, Alarcon GS, Bocanegra TS, Carrillo C, Guerra JC, Rolando I, Espinoza LR. 1982. Articular involvement in human brucellosis: a retrospective analysis of 304 cases. Semin Arthritis Rheum. 12:245-255.

58. Skyberg JA, Thornburg T, Kochetkova I, Layton W, Callis G, Rollins MF, Riccardi C, Becker T, Golden S, Pascual DW. 2012. IFN-y-deficient mice develop IL-1-dependent cutaneous and musculoskeletal inflammation during experimental brucellosis. J Leukoc Biol. 92:375-387.

59. Yagupsky P, Peled N. 2002. Use of the Isolator 1.5 microbial tube for detection of Brucella melitensis in synovial fluid. J Clin Microbiol. 40:3878-3878.

60. Shaalan MA, Memish ZA, Mahmoud SA, Alomari A, Khan MY, Almuneef M, Alalola S. 2002. Brucellosis in children: clinical observations in 115 cases. Int J Infect Dis. 6:182-186.

61. al-Eissa YA, Kambal AM, Alrabeeah AA, Abdullah AM, al-Jurayyan NA, alJishi NM. 1990. Osteoarticular brucellosis in children. Ann Rheum Dis. 49:896900.

62. Madkour MM. 1989. Bone and joint brucellosis, p90-104. In Madkour (ed). Brucellosis. Butterworths, London.

63. Ayaşlıoğlu E, Özlük Ö, Kılıç D, Kaygusuz S, Kara S, Aydın G, Çokca F, Tekeli E. 2005. A case of brucellar septic arthritis of the knee with a prolonged clinical course. Rheumatol Internat. 25:69-71.

64. LAAJAM MA. 1985. Synovial rupture complicating Brucella arthritis. Rheumatol. 24:191-193.

65. Kelly PJ, Martin WJ, Schirger A, Weed LA. 1960. Brucellosis of the bones and joints: experience with thirty-six patients. JAMA. 174:347-353.

66. Wallach JC, Delpino MV, Scian R, Deodato B, Fossati CA, Baldi PC. 2010. Prepatellar bursitis due to Brucella abortus: case report and analysis of the local immune response. J Med Microbiol. 59:1514-1518. 
67. Martin-Hernandez C. B-JJ, Espallargas-Doñate T., Fuertes-Vallcorba A. . 2009. Arthroscopic synovectomy, an alternative in the treatment of brucellar arthritis of the knee with prolonged course. A report of two cases. Int J Orthopedic Surg. 13.

68. Pourbagher A, Pourbagher MA, Savas L, Turunc T, Demiroglu YZ, Erol I, Yalcintas D. 2006. Epidemiologic, clinical, and imaging findings in brucellosis patients with osteoarticular involvement. Am J Roentgenol.187:873-880.

69. Tekkök IH, Berker M, Özcan OE, Özgen T, Akalin E. 1993. Brucellosis of the spine. Neurosurgery 33:838-844.

70. Lacey CA, Keleher LL, Mitchell WJ, Brown CR, Skyberg JA. 2016. CXCR2 mediates Brucella-induced arthritis in interferon gamma-deficient mice. J Infect Dis. 214:151-60.

71. Bosseray N, Plommet M, De Rycke J. 1982. Evolution de l'infection de la souris par Brucella abortus, Brucella melitensis et Brucella suis vers l'état chronique et la guérison, Ann. Rech. Vet. 13:153-161.

72. Smither SJ, Perkins SD, Davies C, Stagg AJ, Nelson M, Atkins HS. 2009. Development and characterization of mouse models of infection with aerosolized Brucella melitensis and Brucella suis. Clin Vaccine Immunol. 16:779-783.

73. Grilló M-J, Blasco JM, Gorvel JP, Moriyón I, Moreno E. 2012. What have we learned from brucellosis in the mouse model? Vet Res. 43:29.

74. Johnson B, Mosier D, Morton R, Confer A. 1994. Experimental Brucella abortus strain 19 arthritis in young cattle. J Vet Diagn Invest. 6:56-61.

75. Magnani DM, Lyons ET, Forde TS, Shekhani MT, Adarichev VA, Splitter GA. 2013. Osteoarticular tissue infection and development of skeletal pathology in murine brucellosis. Dis Model Mech. 6:811-818.

76. Medzhitov R. 2010. Inflammation 2010: new adventures of an old flame. Cell. 140:771-776.

77. Pober JS, Sessa WC. 2007. Evolving functions of endothelial cells in inflammation. Nat Rev Immunol. 7:803-815.

78. Griffith JW, Sokol CL, Luster AD. 2014. Chemokines and chemokine receptors: positioning cells for host defense and immunity. Annu Rev Immunol. 32:659-702.

79. Petri B, Bixel MG. 2006. Molecular events during leukocyte diapedesis. FEBS J. 273:4399-4407. 
80. Amulic B, Cazalet C, Hayes GL, Metzler KD, Zychlinsky A. 2012. Neutrophil Function: From Mechanisms to Disease. Annu Rev Immunol 30:459-489.

81. Kim ND, Chou RC, Seung E, Tager AM, Luster AD. 2006. A unique requirement for the leukotriene B4 receptor BLT1 for neutrophil recruitment in inflammatory arthritis. J Exp Med. 203:829-835.

82. Chen M, Lam BK, Kanaoka Y, Nigrovic PA, Audoly LP, Austen KF, Lee DM. 2006. Neutrophil-derived leukotriene B4 is required for inflammatory arthritis. J Exp Med. 203:837-842.

83. Zawrotniak M, Rapala-Kozik M. 2013. Neutrophil extracellular traps (NETs) formation and implications. Acta Biochim Pol. 60:277-284.

84. Bennouna S, Bliss SK, Curiel TJ, Denkers EY. 2003. Cross-talk in the innate immune system: neutrophils instruct recruitment and activation of dendritic cells during microbial infection. J Immunol. 171:6052-6058.

85. Manfredi AA, Ramirez GA, Rovere-Querini P, Maugeri N. 2018. The neutrophil's choice: phagocytose vs make neutrophil extracellular traps. Front Immunol. 9:288.

86. Amer AO, Swanson MS. 2002. A phagosome of one's own: a microbial guide to life in the macrophage. Curr Opin Microbiol. 5:56-61.

87. Fang FC. 2004. Antimicrobial reactive oxygen and nitrogen species: concepts and controversies. Nat Rev Microbiol. 2:820.

88. Serbina NV, Jia T, Hohl TM, Pamer EG. 2008. Monocyte-mediated defense against microbial pathogens. Annu Rev Immunol. 26:421-452.

89. Hamidzadeh K, Christensen SM, Dalby E, Chandrasekaran P, Mosser DM. 2017. Macrophages and the recovery from acute and chronic inflammation. Annu Rev Physiol. 79:567-592.

90. Erwig LP, Henson PM. 2007. Immunological consequences of apoptotic cell phagocytosis. Am J Pathol. 171:2-8.

91. Mayadas TN, Cullere X, Lowell CA. 2014. The multifaceted functions of neutrophils. Ann Rev of Pathol. 9:181-218.

92. Baldi PC, Giambartolomei GH. 2013. Immunopathology of Brucella infection. Recent Pat Antiinfect Drug Discov. 8:18-26.

93. Anderson T, Cheville N, Meador V. 1986. Pathogenesis of placentitis in the goat inoculated with Brucella abortus. II. Ultrastructural studies. Vet pathol. 23:227-239. 
94. Watarai M, Makino Si, Fujii Y, Okamoto K, Shirahata T. 2002. Modulation of Brucella-induced macropinocytosis by lipid rafts mediates intracellular replication. Cell Microbiol. 4:341-355.

95. Gorvel JP, Moreno E. 2002. Brucella intracellular life: from invasion to intracellular replication. Vet Microbiol. 90:281-297.

96. von Bargen K, Gorvel J-P, Salcedo SP. 2012. Internal affairs: investigating the Brucella intracellular lifestyle. FEMS Microbiol Rev. 36:533-562.

97. Pizarro-Cerdá J, Moreno E, Gorvel J-P. 2000. Invasion and intracellular trafficking of Brucella abortus in nonphagocytic cells. Microbes Infect. 2:829-835.

98. Boschiroli ML, Ouahrani-Bettache S, Foulongne V, Michaux-Charachon S, Bourg G, Allardet-Servent A, Cazevieille C, Liautard JP, Ramuz M, O'Callaghan D. 2002. The Brucella suis virB operon is induced intracellularly in macrophages. Proc Natl Acad Sci. 99:1544-1549.

99. Celli J, de Chastellier C, Franchini DM, Pizarro-Cerda J, Moreno E, Gorvel JP. 2003. Brucella evades macrophage killing via VirB-dependent sustained interactions with the endoplasmic reticulum. J Exp Med. 198:545-556.

100. Xavier MN, Winter MG, Spees AM, den Hartigh AB, Nguyen K, Roux CM, Silva TM, Atluri VL, Kerrinnes T, Keestra AM, Monack DM, Luciw PA, Eigenheer RA, Baumler AJ, Santos RL, Tsolis RM. 2013. PPARgamma-mediated increase in glucose availability sustains chronic Brucella abortus infection in alternatively activated macrophages. Cell Host Microbe. 14:159-170.

101. Czyz DM, Willett JW, Crosson S. 2017. Brucella abortus Induces a Warburg shift in host metabolism that is linked to enhanced intracellular survival of the pathogen. J Bacteriol. 199.

102. Lawrence T, Natoli G. 2011. Transcriptional regulation of macrophage polarization: enabling diversity with identity. Nat Rev Immunol. 11:750-761.

103. Galván-Peña S, O'Neill LA. 2014. Metabolic reprograming in macrophage polarization. Front Immunol. 5:420.

104. Price JV, Vance RE. 2014. The macrophage paradox. Immunity. 41:685-693.

105. West AP, Koblansky AA, Ghosh S. 2006. Recognition and signaling by Toll-Like receptors. Annu Rev Cell Dev Biol. 22:409-437.

106. Chaudhary A, Ganguly K, Cabantous S, Waldo GS, Micheva-Viteva SN, Nag K, Hlavacek WS, Tung C-S. 2012. The Brucella TIR-like protein TcpB interacts with the death domain of MyD88. Biochem BiophysI Res Commun. 417:299-304. 
107. Radhakrishnan GK, Yu Q, Harms JS, Splitter GA. 2009. Brucella TIR domaincontaining protein mimics properties of the Toll-like receptor adaptor protein TIRAP. J Biol Chem. 284:9892-9898.

108. Ferrero MC, Hielpos MS, Carvalho NB, Barrionuevo P, Corsetti PP, Giambartolomei GH, Oliveira SC, Baldi PC. 2014. Key role of Toll-like receptor 2 in the inflammatory response and major histocompatibility complex class ii downregulation in Brucella abortus-infected alveolar macrophages. Infect Immun. 82:626-639.

109. Gomes MT, Campos PC, Pereira GdS, Bartholomeu DC, Splitter G, Oliveira SC. 2016. TLR9 is required for MAPK/NF-KB activation but does not cooperate with TLR2 or TLR6 to induce host resistance to Brucella abortus. J Leukoc Biol. 99:771-780.

110. de Almeida LA, Macedo GC, Marinho FA, Gomes MT, Corsetti PP, Silva AM, Cassataro J, Giambartolomei GH, Oliveira SC. 2013. Toll-like receptor 6 plays an important role in host innate resistance to Brucella abortus infection in mice. Infect Immun. 81:1654-1662.

111. Campos MA, Rosinha GM, Almeida IC, Salgueiro XS, Jarvis BW, Splitter GA, Qureshi N, Bruna-Romero O, Gazzinelli RT, Oliveira SC. 2004. Role of Toll-like receptor 4 in induction of cell-mediated immunity and resistance to Brucella abortus infection in mice. Infect Immun. 72:176-186.

112. Vieira ALS, Silva TM, Mol JP, Oliveira SC, Santos RL, Paixão TA. 2013. MyD88 and TLR9 are required for early control of Brucella ovis infection in mice. Res Vet Sci. 94:399-405.

113. Kawai T, Akira S. 2005. Toll-like receptor downstream signaling. Arthritis Res Ther. 7:12-19.

114. Chen Z, Su L, Xu Q, Katz J, Michalek SM, Fan M, Feng X, Zhang P. 2015. IL1R/TLR2 through MyD88 divergently modulates osteoclastogenesis through regulation of nuclear factor of activated T cells c1 (NFATc1) and B lymphocyteinduced maturation protein-1 (Blimp1). J Biol Chem. 290:30163-30174.

115. Kyo F, Futani H, Matsui K, Terada M, Adachi K, Nagata K, Sano H, Tateishi H, Tsutsui H, Nakanishi K. 2005. Endogenous Interleukin-6, but not tumor necrosis factor $\alpha$, contributes to the development of Toll-like receptor $4 /$ myeloid differentiation factor 88-mediated acute arthritis in mice. Arthritis Rheum. 52:2530-2540.

116. Joosten LA, Koenders MI, Smeets RL, Heuvelmans-Jacobs M, Helsen MM, Takeda K, Akira S, Lubberts E, van de Loo FA, van den Berg WB. 2003. Tolllike receptor 2 pathway drives streptococcal cell wall-induced joint inflammation: critical role of myeloid differentiation factor 88 . J Immunol. 171:6145-6153. 
117. Gomes MT, Campos PC, Oliveira FS, Corsetti PP, Bortoluci KR, Cunha LD, Zamboni DS, Oliveira SC. 2013. Critical role of ASC inflammasomes and bacterial type IV secretion system in caspase-1 activation and host innate resistance to Brucella abortus infection. J Immunol. 190:3629-3638.

118. Franco MMC, Marim F, Guimarães ES, Assis NR, Cerqueira DM, Alves-Silva J, Harms J, Splitter G, Smith J, Kanneganti T-D. 2018. Brucella abortus triggers a cGAS-Independent STING pathway to induce host protection that involves guanylate-binding proteins and inflammasome activation. J Immunol. 200:607622.

119. Man SM, Place DE, Kuriakose T, Kanneganti TD. 2017. Interferon-inducible guanylate-binding proteins at the interface of cell-autonomous immunity and inflammasome activation. J Leukoc Biol. 101:143-150.

120. Rathinam VA, Fitzgerald KA. 2016. Inflammasome Complexes: emerging mechanisms and effector functions. Cell. 165:792-800.

121. Ruhl S, Broz P. 2015. Caspase-11 activates a canonical NLRP3 inflammasome by promoting K(+) efflux. Eur J Immunol. 45:2927-2936.

122. Jakka P, Namani S, Murugan S, Rai N, Radhakrishnan G. 2017. The Brucella effector protein TcpB induces degradation of inflammatory caspases and thereby subverts noncanonical inflammasome activation in macrophages. J Biol Chem. 292(50):20613-20627.

123. Barquero-Calvo E, Chaves-Olarte E, Weiss DS, Guzmán-Verri C, ChacónDíaz C, Rucavado A, Moriyón I, Moreno E. 2007. Brucella abortus uses a stealthy strategy to avoid activation of the innate immune system during the onset of infection. PloS one 2:e631.

124. Lapaque N, Moriyon I, Moreno E, Gorvel J-P. 2005. Brucella lipopolysaccharide acts as a virulence factor. Curr Opin Microbiol. 8:60-66.

125. Conde-Álvarez R, Arce-Gorvel V, Iriarte M, Manček-Keber M, Barquero-Calvo E, Palacios-Chaves L, Chacón-Díaz C, Chaves-Olarte E, Martirosyan A, von Bargen K. 2012. The lipopolysaccharide core of Brucella abortus acts as a shield against innate immunity recognition. PLoS Pathog. 8:e1002675.

126. Ficht TA, Pei J, Kahl-McDonagh M. 2014. Brucella dissociation is essential for macrophage egress and bacterial dissemination. Front Cell Infect Microbiol. 4:23.

127. Kitsis E, Weissmann G. 1991. The role of the neutrophil in rheumatoid arthritis. Clin Orthop Relat Res. 63-72.

128. Talbot J, Bianchini FJ, Nascimento DC, Oliveira RD, Souto FO, Pinto LG, Peres RS, Silva JR, Almeida SC, Louzada-Junior P, Cunha TM, Cunha FQ, 
Alves-Filho JC. 2015. CCR2 expression in neutrophils plays a critical role in their migration into joints in rheumatoid arthritis. Arthritis Rheumatol. 67:1751-9.

129. Delpino MV, Fossati CA, Baldi PC. 2009. Proinflammatory response of human osteoblastic cell lines and osteoblast-monocyte interaction upon infection with Brucella spp. Infect Immun. 77:984-995.

130. Scian R, Barrionuevo P, Giambartolomei GH, Fossati CA, Baldi PC, Delpino MV. 2010. GM-CSF-and TNF-a-mediated matrix metalloproteinase production by human osteoblasts and monocytes upon infection with Brucella abortus. Infect Immun. 79:192-202.

131. Borish LC, Steinke JW. 2003. 2. Cytokines and chemokines. J of Allergy Clin Immunol. 111:S460-S475.

132. Firestein GS. 2003. Evolving concepts of rheumatoid arthritis. Nature. 423:356.

133. Dinarello CA. 2009. Immunological and inflammatory functions of the Interleukin1 Family. Annu Rev Immunol. 27:519-550.

134. Maltez VI, Tubbs AL, Cook KD, Aachoui Y, Falcone EL, Holland SM, Whitmire JK, Miao EA. 2015. Inflammasomes coordinate pyroptosis and natural killer cell cytotoxicity to clear infection by a ubiquitous environmental bacterium. Immunity 43:987-997.

135. Zhan Y, Stanley E, Cheers C. 1991. Prophylaxis or treatment of experimental brucellosis with Interleukin-1. Infect Immun. 59:1790-1794.

136. Gracie JA, Forsey RJ, Chan WL, Gilmour A, Leung BP, Greer MR, Kennedy K, Carter R, Wei XQ, Xu D, Field M, Foulis A, Liew FY, Mclnnes IB. 1999. A proinflammatory role for IL-18 in rheumatoid arthritis. J Clin Invest. 104:1393-1401.

137. Bresnihan B, Alvaro-Gracia JM, Cobby M, Doherty M, Domljan Z, Emery P, Nuki G, Pavelka K, Rau R, Rozman B. 1998. Treatment of rheumatoid arthritis with recombinant human Interleukin-1 receptor antagonist. Arthritis \& Rheumatol. 41:2196-2204.

138. Burger D, Dayer JM, Palmer G, Gabay C. 2006. Is IL-1 a good therapeutic target in the treatment of arthritis? Best Pract Res Clin Rheumatol. 20:879-896.

139. Tissi L, Puliti M, Barluzzi R, Orefici G, von Hunolstein C, Bistoni F. 1999. Role of tumor necrosis factor alpha, Interleukin-1beta, and Interleukin-6 in a mouse model of group B streptococcal arthritis. Infect Immun. 67:4545-4550.

140. Zhan Y, Cheers C. 1993. Endogenous gamma interferon mediates resistance to Brucella abortus infection. Infect Immun. 61:4899-4901. 
141. Murphy EA, Sathiyaseelan J, Parent MA, Zou B, Baldwin CL. 2001. Interferon$Y$ is crucial for surviving a Brucella abortus infection in both resistant C57BL/6 and susceptible BALB/c mice. Immunology 103:511-518.

142. Hanot Mambres D, Machelart A, Potemberg G, De Trez C, Ryffel B, Letesson JJ, Muraille E. 2016. Identification of immune effectors essential to the control of primary and secondary intranasal infection with Brucella melitensis in mice. J Immunol. 196:3780-3793.

143. Jiang X, Leonard B, Benson R, Baldwin CL. 1993. Macrophage control of Brucella abortus: role of reactive oxygen intermediates and nitric oxide. Cell Immunol. 151:309-319.

144. Hedayatizadeh-Omran A, Rafiei A, Hajilooi M, Haghshenas M. 2010. Interferon-gamma low producer genotype +5644 over presented in patients with focal brucellosis. Pak J Biol Sci. 13:1036-1041.

145. Bravo MJ, de Dios Colmenero J, Alonso A, Caballero A. 2003. Polymorphisms of the interferon gamma and Interleukin 10 genes in human brucellosis. Eur $\mathrm{J}$ Immunogenet. 30:433-435.

146. Rasouli M, Kiany S. 2007. Association of interferon-gamma and Interleukin-4 gene polymorphisms with susceptibility to brucellosis in Iranian patients. Cytokine 38:49-53.

147. Eskandari-Nasab E, Moghadampour M, Hasani SS, Hadadi-fishani M, Mirghanizadeh-Bafghi SA, Asadi-Saghandi A, Zare F, Sadeghi-Kalani B, Ghazali-bina M. 2013. Relationship between $\mathrm{Y}$-interferon gene polymorphisms and susceptibility to brucellosis infection. Microbiol Immunol. 57:785-791.

148. Rafiei A, Ardestani SK, Kariminia A, Keyhani A, Mohraz M, Amirkhani A. 2006. Dominant Th1 cytokine production in early onset of human brucellosis followed by switching towards Th2 along prolongation of disease. J Infect. 53:315-324.

149. Durward-Diioia M, Harms J, Khan M, Hall C, Smith JA, Splitter GA. 2015. CD8+ $T$ cell exhaustion, suppressed gamma interferon production, and delayed memory response induced by chronic Brucella melitensis infection. Infect immun. 83:47594771.

150. Zhang T, Kawakami K, Qureshi MH, Okamura H, Kurimoto M, Saito A. 1997. Interleukin-12 (IL-12) and IL-18 synergistically induce the fungicidal activity of murine peritoneal exudate cells against Cryptococcus neoformans through production of gamma interferon by natural killer cells. Infect Immun. 65:3594-3599.

151. Robinson D, Shibuya K, Mui A, Zonin F, Murphy E, Sana T, Hartley SB, Menon S, Kastelein R, Bazan F. 1997. IGIF does not drive Th1 development but 
synergizes with IL-12 for interferon- $\mathrm{y}$ production and activates IRAK and NFKB. Immunity. 7:571-581.

152. Gao L-Y, Kwaik YA. 2000. The modulation of host cell apoptosis by intracellular bacterial pathogens. Trends Microbiol. 8:306-313.

153. Delpino MV, Barrionuevo P, Macedo GC, Oliveira SC, Genaro SD, Scian R, Miraglia MC, Fossati CA, Baldi PC, Giambartolomei GH. 2012. Macrophageelicited osteoclastogenesis in response to Brucella abortus infection requires TLR2/MyD88-dependent TNF-alpha production. J Leukoc Biol. 91:285-298.

154. Gross A, Terraza A, Ouahrani-Bettache S, Liautard J-P, Dornand J. 2000. In vitro Brucella suis infection prevents the programmed cell death of human monocytic cells. Infect Immun. 68:342-351.

155. Scian R, Barrionuevo P, Rodriguez AM, Arriola Benitez PC, Garcia Samartino C, Fossati CA, Giambartolomei GH, Delpino MV. 2013. Brucella abortus invasion of synoviocytes inhibits apoptosis and induces bone resorption through RANKL expression. Infect Immun. 81:1940-1951.

156. Mor A, Abramson SB, Pillinger MH. 2005. The fibroblast-like synovial cell in rheumatoid arthritis: a key player in inflammation and joint destruction. Clin Immunol. 115:118-128.

157. Mancilla M. 2016. Smooth to rough dissociation in Brucella: the missing link to virulence. Front Cell Infect Microbiol. 5:98.

158. England JC, Perchuk BS, Laub MT, Gober JW. 2010. Global regulation of gene expression and cell differentiation in Caulobacter crescentus in response to nutrient availability. J Bacteriol. 192:819-833.

159. Kovach ME, Elzer PH, Hill DS, Robertson GT, Farris MA, Roop RM, Peterson KM. 1995. Four new derivatives of the broad-host-range cloning vector pBBR1MCS, carrying different antibiotic-resistance cassettes. Gene. 166:175176.

160. Lacey CA, Mitchell WJ, Brown CR, Skyberg JA. 2017. Temporal role for MyD88 in a model of Brucella-induced arthritis and musculoskeletal inflammation. Infect Immun. 85.

161. Mishra BB, Rathinam VA, Martens GW, Martinot AJ, Kornfeld H, Fitzgerald KA, Sassetti CM. 2013. Nitric oxide controls the immunopathology of tuberculosis by inhibiting NLRP3 inflammasome-dependent processing of IL-1beta. Nat Immunol. 14:52-60. 
162. Nielsen K, Kelly L, Mallory M. 1998. Standardization of smooth lipopolysaccharide preparations for use in diagnostic serological tests for bovine antibody Brucella abortus. J Immunoassay. 19:239-250.

163. Skyberg JA, Thornburg T, Rollins M, Huarte E, Jutila MA, Pascual DW. 2011. Murine and bovine gammadelta T cells enhance innate immunity against Brucella abortus infections. PLoS One. 6:e21978.

164. Schmittgen TD, Livak KJ. 2008. Analyzing real-time PCR data by the comparative C T method. Nat Protoc. 3:1101.

165. Skyberg JA, Thornburg T, Kochetkova I, Layton W, Callis G, Rollins MF, Riccardi C, Becker T, Golden S, Pascual DW. 2012. IFN-gamma-deficient mice develop IL-1-dependent cutaneous and musculoskeletal inflammation during experimental brucellosis. J Leukoc Biol. 92:375-387.

166. Jimenez de Bagues MP, Dudal S, Dornand J, Gross A. 2005. Cellular bioterrorism: how Brucella corrupts macrophage physiology to promote invasion and proliferation. Clin Immunol. 114:227-238.

167. Press J, Peled N, Buskila D, Yagupsky P. 2002. Leukocyte count in the synovial fluid of children with culture-proven brucellar arthritis. Clin Rheumatol. 21:191-193.

168. Jung H, Mithal DS, Park JE, Miller RJ. 2015. Localized CCR2 activation in the bone marrow niche mobilizes monocytes by desensitizing CXCR4. PLoS One. 10:e0128387.

169. Russo RC, Garcia CC, Teixeira MM, Amaral FA. 2014. The CXCL8/IL-8 chemokine family and its receptors in inflammatory diseases. Expert Rev Clin Immunol. 10:593-619.

170. Corsiero E, Pitzalis C, Bombardieri M. 2014. Peripheral and synovial mechanisms of humoral autoimmunity in rheumatoid arthritis. Drug Discov Today. 19:1161-1165.

171. Mellado M, Martinez-Munoz L, Cascio G, Lucas P, Pablos JL, RodriguezFrade JM. 2015. T cell migration in rheumatoid arthritis. Front Immunol. 6:384.

172. Brown CR, Reiner SL. 1999. Genetic control of experimental lyme arthritis in the absence of specific immunity. Infect Immun. 67:1967-1973.

173. Izadjoo MJ, Polotsky Y, Mense MG, Bhattacharjee AK, Paranavitana CM, Hadfield TL, Hoover DL. 2000. Impaired control of Brucella melitensis infection in Rag1-deficient mice. Infect Immun. 68:5314-5320. 
174. Goenka R, Parent MA, Elzer PH, Baldwin CL. 2011. B cell-deficient mice display markedly enhanced resistance to the intracellular bacterium Brucella abortus. J Infect Dis. 203:1136-1146.

175. Vitry MA, De Trez C, Goriely S, Dumoutier L, Akira S, Ryffel B, Carlier Y, Letesson JJ, Muraille E. 2012. Crucial role of gamma interferon-producing CD4+ Th1 cells but dispensable function of CD8+ T cell, B cell, Th2, and Th17 responses in the control of Brucella melitensis infection in mice. Infect Immun. 80:4271-4280.

176. Clapp B, Yang X, Thornburg T, Walters N, Pascual DW. 2016. Nasal vaccination stimulates CD8 T cells for potent protection against mucosal Brucella melitensis challenge. Immunol Cell Biol. 94: 496-508.

177. Quinones MP, Jimenez F, Martinez H, Estrada CA, Willmon O, Dudley M, Kuziel WA, Melby PC, Reddick RL, Ahuja SK, Ahuja SS. 2006. CC chemokine receptor (CCR)-2 prevents arthritis development following infection by Mycobacterium avium. J Mol Med (Berl). 84:503-512.

178. Brown CR, Blaho VA, Loiacono CM. 2003. Susceptibility to experimental Lyme arthritis correlates with KC and monocyte chemoattractant protein-1 production in joints and requires neutrophil recruitment via CXCR2. J Immunol. 171:893-901.

179. Kruger P, Saffarzadeh M, Weber AN, Rieber N, Radsak M, von Bernuth H, Benarafa C, Roos D, Skokowa J, Hartl D. 2015. Neutrophils: Between host defence, immune modulation, and tissue injury. PLoS Pathog. 11:e1004651.

180. Kobayashi Y. 2008. The role of chemokines in neutrophil biology. Front Biosci. 13:2400-2407.

181. Barquero-Calvo E, Martirosyan A, Ordonez-Rueda D, Arce-Gorvel V, AlfaroAlarcon A, Lepidi H, Malissen B, Malissen M, Gorvel JP, Moreno E. 2013. Neutrophils exert a suppressive effect on Th1 responses to intracellular pathogen Brucella abortus. PLoS Pathog. 9:e1003167.

182. Wallach JC, Delpino MV, Scian R, Deodato B, Fossati CA, Baldi PC. 2010. Prepatellar bursitis due to Brucella abortus: case report and analysis of the local immune response. J Med Microbiol. 59:1514-1518.

183. Wipke BT, Allen PM. 2001. Essential role of neutrophils in the initiation and progression of a murine model of rheumatoid arthritis. J Immunol. 167:1601-1608.

184. Tanaka D, Kagari T, Doi H, Shimozato T. 2006. Essential role of neutrophils in anti-type II collagen antibody and lipopolysaccharide-induced arthritis. Immunology. 119:195-202.

185. Brown CR, Blaho VA, Loiacono CM. 2004. Treatment of mice with the neutrophildepleting antibody RB6-8C5 results in early development of experimental lyme 
arthritis via the recruitment of Gr-1- polymorphonuclear leukocyte-like cells. Infect Immun. 72:4956-4965.

186. Ramos CD, Fernandes KS, Canetti C, Teixeira MM, Silva JS, Cunha FQ. 2006. Neutrophil recruitment in immunized mice depends on MIP-2 inducing the sequential release of MIP-1alpha, TNF-alpha and LTB(4). Eur J Immunol. 36:2025-2034.

187. Scian R, Barrionuevo P, Giambartolomei GH, De Simone EA, Vanzulli SI, Fossati CA, Baldi PC, Delpino MV. 2011. Potential role of fibroblast-like synoviocytes in joint damage induced by Brucella abortus infection through production and induction of matrix metalloproteinases. Infect Immun. 79:36193632.

188. Ritzman AM, Hughes-Hanks JM, Blaho VA, Wax LE, Mitchell WJ, Brown CR. 2010. The chemokine receptor CXCR2 ligand KC (CXCL1) mediates neutrophil recruitment and is critical for development of experimental Lyme arthritis and carditis. Infect Immun. 78:4593-4600.

189. Barsante MM, Cunha TM, Allegretti M, Cattani F, Policani F, Bizzarri C, Tafuri WL, Poole S, Cunha FQ, Bertini R, Teixeira MM. 2008. Blockade of the chemokine receptor CXCR2 ameliorates adjuvant-induced arthritis in rats. $\mathrm{Br} \mathrm{J}$ Pharmacol. 153:992-1002.

190. Podolin PL, Bolognese BJ, Foley JJ, Schmidt DB, Buckley PT, Widdowson KL, Jin Q, White JR, Lee JM, Goodman RB, Hagen TR, Kajikawa O, Marshall LA, Hay DW, Sarau HM. 2002. A potent and selective nonpeptide antagonist of CXCR2 inhibits acute and chronic models of arthritis in the rabbit. J Immunol. 169:6435-6444.

191. Jacobs JP, Ortiz-Lopez A, Campbell JJ, Gerard CJ, Mathis D, Benoist C. 2010. Deficiency of CXCR2, but not other chemokine receptors, attenuates autoantibody-mediated arthritis in a murine model. Arthritis Rheum. 62:1921-1932.

192. Coelho FM, Pinho V, Amaral FA, Sachs D, Costa VV, Rodrigues DH, Vieira AT, Silva TA, Souza DG, Bertini R, Teixeira AL, Teixeira MM. 2008. The chemokine receptors CXCR1/CXCR2 modulate antigen-induced arthritis by regulating adhesion of neutrophils to the synovial microvasculature. Arthritis Rheum. 58:2329-2337.

193. Morrison TE, Oko L, Montgomery SA, Whitmore AC, Lotstein AR, Gunn BM, Elmore SA, Heise MT. 2011. A mouse model of chikungunya virus-induced musculoskeletal inflammatory disease: evidence of arthritis, tenosynovitis, myositis, and persistence. Am J Pathol. 178:32-40. 
194. Lee JH. 2011. Involvement of T-cell immunoregulation by ochnaflavone in therapeutic effect on fungal arthritis due to Candida albicans. Arch Pharm Res. 34:1209-1217.

195. Adachi O, Kawai T, Takeda K, Matsumoto M, Tsutsui H, Sakagami M, Nakanishi K, Akira S. 1998. Targeted disruption of the MyD88 gene results in loss of IL-1- and IL-18-mediated function. Immunity. 9:143-150.

196. Miller LS, O'Connell RM, Gutierrez MA, Pietras EM, Shahangian A, Gross CE, Thirumala A, Cheung AL, Cheng G, Modlin RL. 2006. MyD88 mediates neutrophil recruitment initiated by IL-1R but not TLR2 activation in immunity against Staphylococcus aureus. Immunity. 24:79-91.

197. Isomäki P, Punnonen J. 1997. Pro-and anti-inflammatory cytokines in rheumatoid arthritis. Ann Med. 29:499-507.

198. Tarkowski A. 2006. Infectious arthritis. Best Pract Res Clin Rheumatol. 20:10291044.

199. Bosseray N. 1980. Colonization of mouse placentas by Brucella abortus inoculated during pregnancy. Br J Exp Pathol. 61:361.

200. Bosseray N, Plommet M. 1990. Brucella suis S2, Brucella melitensis Rev. 1 and Brucella abortus S19 living vaccines: residual virulence and immunity induced against three Brucella species challenge strains in mice. Vaccine 8:462-468.

201. Plommet M, Plommet A-M. 1983. Immune serum-mediated effects on brucellosis evolution in mice. Infect Immun. 41:97-105.

202. Pardon P. Resistance against a subcutaneous Brucella challenge of mice immunized with living or dead Brucella or by transfer of immune serum. Ann Immnol (Paris). 128: 1025-37.

203. Weiss DS, Takeda K, Akira S, Zychlinsky A, Moreno E. 2005. MyD88, but not Toll-like receptors 4 and 2, is required for efficient clearance of Brucella abortus. Infect Immun. 73:5137-5143.

204. Macedo GC, Magnani DM, Carvalho NB, Bruna-Romero O, Gazzinelli RT, Oliveira SC. 2008. Central role of MyD88-dependent dendritic cell maturation and proinflammatory cytokine production to control Brucella abortus infection. J Immunol. 180:1080-1087.

205. Copin R, De Baetselier P, Carlier Y, Letesson JJ, Muraille E. 2007. MyD88dependent activation of B220-CD11b+LY-6C+ dendritic cells during Brucella melitensis infection. J Immunol. 178:5182-5191. 
206. Foell D, Wittkowski H, Roth J. 2007. Mechanisms of disease: a'DAMP'view of inflammatory arthritis. Nat Rev Rheumatol. 3:382.

207. Keleher LL, Skyberg JA. 2016. Activation of bovine neutrophils by Brucella spp. Vet Immunol Immunopathol. 177:1-6.

208. Liu N, Montgomery RR, Barthold SW, Bockenstedt LK. 2004. Myeloid differentiation antigen 88 deficiency impairs pathogen clearance but does not alter inflammation in Borrelia burgdorferi-infected mice. Infect Immun. 72:3195-3203.

209. Bolz DD, Sundsbak RS, Ma Y, Akira S, Kirschning CJ, Zachary JF, Weis JH, Weis JJ. 2004. MyD88 plays a unique role in host defense but not arthritis development in Lyme disease. J Immunol. 173:2003-2010.

210. Abdollahi-Roodsaz S, Joosten LA, Roelofs MF, Radstake TR, Matera G, Popa C, van der Meer JW, Netea MG, van den Berg WB. 2007. Inhibition of Toll-like receptor 4 breaks the inflammatory loop in autoimmune destructive arthritis. Arthritis Rheum. 56:2957-2967.

211. Surendran N, Hiltbold EM, Heid B, Akira S, Standiford TJ, Sriranganathan N, Boyle SM, Zimmerman KL, Makris MR, Witonsky SG. 2012. Role of TLRs in Brucella mediated murine DC activation in vitro and clearance of pulmonary infection in vivo. Vaccine. 30:1502-1512.

212. Nair A, Kanda V, Bush-Joseph C, Verma N, Chubinskaya S, Mikecz K, Glant TT, Malfait AM, Crow MK, Spear GT, Finnegan A, Scanzello CR. 2012. Synovial fluid from patients with early osteoarthritis modulates fibroblast-like synoviocyte responses to Toll-like receptor 4 and Toll-like receptor 2 ligands via soluble CD14. Arthritis Rheum. 64:2268-2277.

213. Liu-Bryan R, Pritzker K, Firestein GS, Terkeltaub R. 2005. TLR2 signaling in chondrocytes drives calcium pyrophosphate dihydrate and monosodium urate crystal-induced nitric oxide generation. J Immunol. 174:5016-5023.

214. Storek KM, Monack DM. 2015. Bacterial recognition pathways that lead to inflammasome activation. Immunol Rev. 265:112-129.

215. Jorgensen I, Zhang Y, Krantz BA, Miao EA. 2016. Pyroptosis triggers poreinduced intracellular traps (PITs) that capture bacteria and lead to their clearance by efferocytosis. J Exp Med. 213:2113-2128.

216. Miller LS, Pietras EM, Uricchio LH, Hirano K, Rao S, Lin H, O'Connell RM, Iwakura Y, Cheung AL, Cheng G. 2007. Inflammasome-mediated production of IL-1 $\beta$ is required for neutrophil recruitment against Staphylococcus aureus in vivo. $\mathrm{J}$ Immunol. 179:6933-6942. 
217. Walle LV, Van Opdenbosch N, Jacques $P$, Fossoul A, Verheugen E, Vogel $P$, Beyaert R, Elewaut D, Kanneganti T-D, van Loo G. 2014. Negative regulation of the NLRP3 inflammasome by A20 protects against arthritis. Nature. 512:69.

218. Bronner DN, Abuaita BH, Chen X, Fitzgerald KA, Nunez G, He Y, Yin XM, O'Riordan MX. 2015. Endoplasmic reticulum stress activates the inflammasome via NLRP3- and Caspase-2-driven mitochondrial damage. Immunity. 43:451-462.

219. Marim FM, Franco MMC, Gomes MTR, Miraglia MC, Giambartolomei GH, Oliveira SC. 2017. The role of NLRP3 and AIM2 in inflammasome activation during Brucella abortus infection. Semin Immunopathol. 39:215-223.

220. Jakobs C, Perner S, Hornung V. 2015. AIM2 drives joint inflammation in a selfDNA triggered model of chronic polyarthritis. PLoS One. 10:e0131702.

221. Zhang Y, Zheng Y, Li H. 2016. NLRP3 inflammasome plays an important role in the pathogenesis of collagen-induced arthritis. Mediators Inflamm. 2016:9656270.

222. von Moltke J, Trinidad NJ, Moayeri M, Kintzer AF, Wang SB, van Rooijen N, Brown CR, Krantz BA, Leppla SH, Gronert K, Vance RE. 2012. Rapid induction of inflammatory lipid mediators by the inflammasome in vivo. Nature. 490:107-111.

223. Labbe K, Saleh M. 2008. Cell death in the host response to infection. Cell Death Differ. 15:1339.

224. Cardoso PG, Macedo GC, Azevedo V, Oliveira SC. 2006. Brucella spp noncanonical LPS: structure, biosynthesis, and interaction with host immune system. Microb Cell Fact. 5:13.

225. Barbier T, Zuniga-Ripa A, Moussa S, Plovier H, Sternon J, Lazaro-Anton L, Conde-Álvarez R, De Bolle X, Iriarte M, Moriyón I. 2018. Brucella central carbon metabolism: an update. Crit Rev Microbiol. 44:182-211.

226. Rayamajhi M, Zhang Y, Miao EA. 2013. Detection of pyroptosis by measuring released lactate dehydrogenase activity. Methods Mol Biol. 1040:85-90.

227. Shao W, Yeretssian G, Doiron K, Hussain SN, Saleh M. 2007. The caspase-1 digestome identifies the glycolysis pathway as a target during infection and septic shock. J Biol Chem. 282:36321-36329.

228. Skyberg JA, Lacey CA. 2017. Hematopoietic MyD88 and IL-18 are essential for IFN-gamma-dependent restriction of type A Francisella tularensis infection. J Leukoc Biol. 102:1441-1450.

229. Caligiuri MA. 2008. Human natural killer cells. Blood. 112:461-469. 
230. Hernandez-Cuellar E, Tsuchiya K, Hara H, Fang R, Sakai S, Kawamura I, Akira S, Mitsuyama M. 2012. Cutting edge: nitric oxide inhibits the NLRP3 inflammasome. J Immunol. 189:5113-5117.

231. Kim YM, Talanian RV, Li J, Billiar TR. 1998. Nitric oxide prevents IL-1beta and IFN-gamma-inducing factor (IL-18) release from macrophages by inhibiting caspase-1 (IL-1beta-converting enzyme). J Immunol. 161:4122-4128.

232. Hagar JA, Powell DA, Aachoui Y, Ernst RK, Miao EA. 2013. Cytoplasmic LPS activates caspase-11: implications in TLR4-independent endotoxic shock. Science. 341:1250-1253.

233. Shi J, Zhao Y, Wang Y, Gao W, Ding J, Li P, Hu L, Shao F. 2014. Inflammatory caspases are innate immune receptors for intracellular LPS. Nature. 514:187.

234. Wang W, Shao Y, Li S, Xin N, Ma T, Zhao C, Song M. 2017. Caspase-11 plays a protective role in pulmonary acinetobacter baumannii Infection. Infect Immun. 85:e00350-00317.

235. Boll JM, Tucker AT, Klein DR, Beltran AM, Brodbelt JS, Davies BW, Trent MS. 2015. Reinforcing lipid A acylation on the cell surface of Acinetobacter baumannii promotes cationic antimicrobial peptide resistance and desiccation survival. MBio. 6:e00478-00415.

236. Barquero-Calvo E, Mora-Cartín R, Arce-Gorvel V, Juana L, Chacón-Díaz C, Chaves-Olarte E, Guzmán-Verri C, Buret AG, Gorvel J-P, Moreno E. 2015. Brucella abortus induces the premature death of human neutrophils through the action of its lipopolysaccharide. PLoS Pathog. 11:e1004853.

237. Kayagaki N, Warming S, Lamkanfi M, Walle LV, Louie S, Dong J, Newton K, Qu Y, Liu J, Heldens S. 2011. Non-canonical inflammasome activation targets caspase-11. Nature. 479:117.

238. Gobelet C, Gerster J. 1984. Synovial fluid lactate levels in septic and non-septic arthritides. Ann Rheum Dis. 43:742-745.

239. Mavridis AK, Drosos AA, Tsolas O, Moutsopoulos HM. 1984. Lactate levels in Brucella arthritis. Rheumatol Int. 4:169-171.

240. Legrand C, Bour JM, Jacob C, Capiaumont J, Martial A, Marc A, Wudtke M, Kretzmer G, Demangel C, Duval D, Hache J. 1992. Lactate dehydrogenase (LDH) activity of the number of dead cells in the medium of cultured eukaryotic cells as marker. J Biotechnol. 25:231-243.

241. Passarella S, de Bari L, Valenti D, Pizzuto R, Paventi G, Atlante A. 2008. Mitochondria and I-lactate metabolism. FEBS Lett. 582:3569-3576. 
242. Tannahill GM, Curtis AM, Adamik J, Palsson-McDermott EM, McGettrick AF, Goel G, Frezza C, Bernard NJ, Kelly B, Foley NH, Zheng L, Gardet A, Tong Z, Jany SS, Corr SC, Haneklaus M, Caffrey BE, Pierce K, Walmsley S, Beasley FC, Cummins E, Nizet V, Whyte M, Taylor CT, Lin H, Masters SL, Gottlieb E, Kelly VP, Clish C, Auron PE, Xavier RJ, O'Neill LA. 2013. Succinate is an inflammatory signal that induces IL-1beta through HIF-1alpha. Nature. 496:238242.

243. Lee DW, Shin HY, Jeong JH, Han J, Ryu S, Nakahira K, Moon JS. 2017. Carbon monoxide regulates glycolysis-dependent NLRP3 inflammasome activation in macrophages. Biochem Biophys Res Commun. 493:957-963.

244. Moon JS, Hisata S, Park MA, DeNicola GM, Ryter SW, Nakahira K, Choi AMK. 2015. mTORC1-Induced HK1-Dependent glycolysis regulates NLRP3 inflammasome activation. Cell Rep. 12:102-115.

245. Mitchell AJ, Yau B, McQuillan JA, Ball HJ, Too LK, Abtin A, Hertzog P, Leib SL, Jones CA, Gerega SK, Weninger W, Hunt NH. 2012. Inflammasomedependent IFN-gamma drives pathogenesis in Streptococcus pneumoniae meningitis. J Immunol. 189:4970-4980.

246. Weizman O-E, Adams NM, Schuster IS, Krishna C, Pritykin Y, Lau C, DegliEsposti MA, Leslie CS, Sun JC, O'Sullivan TE. 2017. ILC1 confer early host protection at initial sites of viral infection. Cell. 171:795-808. e712.

247. Abt MC, Lewis BB, Caballero S, Xiong H, Carter RA, Sušac B, Ling L, Leiner I, Pamer EG. 2015. Innate immune defenses mediated by two ILC subsets are critical for protection against acute Clostridium difficile infection. Cell Host Microbe. 18:27-37.

248. Karki R, Man Si M, Malireddi RKS, Gurung P, Vogel P, Lamkanfi M, Kanneganti T-D. 2015. Concerted activation of the AIM2 and NLRP3 inflammasomes orchestrates host protection against Aspergillus Infection. Cell Host Microbe. 17:357-368.

249. Sengupta R, Holmgren A. 2012. The role of thioredoxin in the regulation of cellular processes by S-nitrosylation. Biochim Biophys Acta. 1820:689-700.

250. Mao K, Chen S, Chen M, Ma Y, Wang Y, Huang B, He Z, Zeng Y, Hu Y, Sun S, Li J, Wu X, Wang X, Strober W, Chen C, Meng G, Sun B. 2013. Nitric oxide suppresses NLRP3 inflammasome activation and protects against LPS-induced septic shock. Cell Res. 23:201-212.

251. Eskandari-Nasab E, Moghadampour M, Hasani SS, Hadadi-fishani M, Mirghanizadeh-Bafghi SA, Asadi-Saghandi A, Zare F, Sadeghi-Kalani B, Ghazali-bina M. 2013. Relationship between gamma-interferon gene 
polymorphisms and susceptibility to brucellosis infection. Microbiol Immunol. 57:785-791.

252. Knox CD, de Kam PJ, Azer K, Wong P, Ederveen AG, Shevell D, Morabito C, Meehan AG, Liu W, Reynders T. 2016. Discovery and clinical evaluation of MK8150 , a novel nitric oxide donor with a unique mechanism of nitric oxide release. $\mathrm{J}$ Am Heart Assoc. 5:e003493.

253. M A Farrar a, Schreiber RD. 1993. The molecular cell biology of interferongamma and its receptor. Ann Rev Immunol. 11:571-611.

254. Stevens MG, Pugh G, Tabatabai LB. 1992. Effects of gamma interferon and indomethacin in preventing Brucella abortus infections in mice. Infect Immun. 60:4407-4409.

255. Felix C, Kaplan Turkoz B, Ranaldi S, Koelblen T, Terradot L, O'Callaghan D, Vergunst AC. 2014. The Brucella TIR domain containing proteins BtpA and BtpB have a structural WxxxE motif important for protection against microtubule depolymerisation. Cell Commun Signal. 12:53.

256. Imboden JB. 2009. The immunopathogenesis of rheumatoid arthritis. Annu Rev Pathol. 4:417-434.

257. Bartok B, Firestein GS. 2010. Fibroblast-like synoviocytes: key effector cells in rheumatoid arthritis. Immunol Rev. 233:233-255.

258. Sweeney SE, Firestein GS. 2004. Rheumatoid arthritis: regulation of synovial inflammation. Int J Biochem Cell Biol. 36:372-378.

259. Tak PP, Bresnihan B. 2000. The pathogenesis and prevention of joint damage in rheumatoid arthritis: advances from synovial biopsy and tissue analysis. Arthritis Rheum. 43:2619-2633.

260. Firestein GS, Yeo M, Zvaifler NJ. 1995. Apoptosis in rheumatoid arthritis synovium. J Clin Invest. 96:1631-1638.

261. Inoue H, Takamori M, Nagata N, Nishikawa T, Oda H, Yamamoto S, Koshihara Y. 2001. An investigation of cell proliferation and soluble mediators induced by Interleukin $1 \beta$ in human synovial fibroblasts: comparative response in osteoarthritis and rheumatoid arthritis. Inflamm Res. 50:65-72.

262. Jimi E, Aoki K, Saito H, D'Acquisto F, May MJ, Nakamura I, Sudo T, Kojima T, Okamoto F, Fukushima H. 2004. Selective inhibition of NF-kB blocks osteoclastogenesis and prevents inflammatory bone destruction in vivo. Nat Med. 10:617-624. 
263. Hardy R, Cooper M. 2009. Bone loss in inflammatory disorders. J Endocrinol. 201:309-320.

264. Nair SP, Meghji S, Wilson M, Reddi K, White P, Henderson B. 1996. Bacterially induced bone destruction: mechanisms and misconceptions. Infect Immun. 64:2371-2380.

265. Fernandes JC, Martel-Pelletier J, Pelletier JP. 2002. The role of cytokines in osteoarthritis pathophysiology. Biorheology. 39:237-246.

266. Morel JC, Park CC, Zhu K, Kumar P, Ruth JH, Koch AE. 2002. Signal transduction pathways involved in rheumatoid arthritis synovial fibroblast Interleukin-18-induced vascular cell adhesion molecule-1 expression. J Biol Chem. 277:34679-34691.

267. Hwang SY, Kim JY, Kim KW, Park MK, Moon Y, Kim WU, Kim HY. 2004. IL-17 induces production of IL-6 and IL-8 in rheumatoid arthritis synovial fibroblasts via NF-kappaB- and PI3-kinase/Akt-dependent pathways. Arthritis Res Ther. 6:R120128.

268. Morel JC, Park CC, Kumar P, Koch AE. 2001. Interleukin-18 induces rheumatoid arthritis synovial fibroblast CXC chemokine production through NFKB activation. Lab Invest. 81:1371.

269. Palmer RMJ, Hickery MS, Charles IG, Moncada S, Bayliss MT. 1993. Induction of nitric oxide synthase in human chondrocytes. Biochem Biophys Res Commun. 193:398-405.

270. Munder M, Mallo M, Eichmann K, Modolell M. 1998. Murine macrophages secrete interferon y upon combined stimulation with Interleukin (IL)-12 and IL-18: a novel pathway of autocrine macrophage activation. J Exp Med. 187:2103-2108.

271. Jones SM, Winter AJ. 1992. Survival of virulent and attenuated strains of Brucella abortus in normal and gamma interferon-activated murine peritoneal macrophages. Infect Immun. 60:3011-3014.

272. Zhan Y, Cheers C. 1995. Endogenous Interleukin-12 is involved in resistance to Brucella abortus infection. Infect immun. 63:1387-1390.

273. Sonnenberg GF, Artis D. 2015. Innate lymphoid cells in the initiation, regulation and resolution of inflammation. Nat Med. 21:698.

274. Lamkanfi M, Sarkar A, Walle LV, Vitari AC, Amer AO, Wewers MD, Tracey KJ, Kanneganti T-D, Dixit VM. 2010. Inflammasome-dependent release of the alarmin HMGB1 in endotoxemia. J Immunol. 185:4385-4392. 
275. Park JS, Svetkauskaite D, He Q, Kim J-Y, Strassheim D, Ishizaka A, Abraham E. 2004. Involvement of Toll-like receptors 2 and 4 in cellular activation by high mobility group box 1 protein. J Biol Chem. 279:7370-7377.

276. Yu M, Wang H, Ding A, Golenbock DT, Latz E, Czura CJ, Fenton MJ, Tracey KJ, Yang H. 2006. HMGB1 signals through Toll-like receptor (TLR) 4 and TLR2. Shock. 26:174-179.

277. Creagh EM, O'Neill LA. 2006. TLRs, NLRs and RLRs: a trinity of pathogen sensors that co-operate in innate immunity. Trends Immunol. 27:352-357.

278. Miao EA, Andersen-Nissen E, Warren SE, Aderem A. TLR5 and Ipaf: dual sensors of bacterial flagellin in the innate immune system. Semin Immunopathol. 29: $275-288$.

279. Pasquali P, Adone R, Gasbarre LC, Pistoia C, Ciuchini F. 2002. Effect of exogenous interleukin-18 (IL-18) and IL-12 in the course of Brucella abortus 2308 infection in mice. Clin Diagn Lab Immunol. 9:491-492.

280. Baldwin C, Winter A. 1994. Macrophages and Brucella. Immunol Ser. 60:363380.

281. Rodriguez-Prados JC, Traves PG, Cuenca J, Rico D, Aragones J, Martin-Sanz P, Cascante M, Bosca L. 2010. Substrate fate in activated macrophages: a comparison between innate, classic, and alternative activation. J Immunol. 185:605-614.

282. Ruscitti P, Cipriani P, Di Benedetto P, Liakouli V, Berardicurti O, Carubbi F, Ciccia F, Alvaro S, Triolo G, Giacomelli R. 2015. Monocytes from patients with rheumatoid arthritis and type 2 diabetes mellitus display an increased production of Interleukin (IL)-1 $\beta$ via the nucleotide-binding domain and leucine-rich repeat containing family pyrin 3 (NLRP3)-inflammasome activation: a possible implication for therapeutic decision in these patients. Clin Exp Immunol. 182:35-44.

283. Garcia-Carbonell R, Divakaruni AS, Lodi A, Vicente-Suarez I, Saha A, Cheroutre H, Boss GR, Tiziani S, Murphy AN, Guma M. 2016. Critical role of glucose metabolism in rheumatoid arthritis fibroblast-like synoviocytes. Arthritis Rheumatol. 68:1614-1626.

284. Leung BP, Culshaw S, Gracie JA, Hunter D, Canetti CA, Campbell C, Cunha F, Liew FY, McInnes IB. 2001. A role for IL-18 in neutrophil activation. J Immunol. 167:2879-2886.

285. De Klerck B, Carpentier I, Lories RJ, Habraken Y, Piette J, Carmeliet G, Beyaert R, Billiau A, Matthys P. 2004. Enhanced osteoclast development in collagen-induced arthritis in interferon-y receptor knock-out mice as related to increased splenic CD11b+ myelopoiesis. Arthritis Res Ther. 6:R220. 
286. Aachoui Y, Kajiwara Y, Leaf IA, Mao D, Ting JP-Y, Coers J, Aderem A, Buxbaum JD, Miao EA. 2015. Canonical inflammasomes drive IFN-y to prime caspase-11 in defense against a cytosol-invasive bacterium. Cell Host Microbe. 18:320-332.

287. Endo M, Mori M, Akira S, Gotoh T. 2006. C/EBP homologous protein (CHOP) is crucial for the induction of caspase-11 and the pathogenesis of lipopolysaccharideinduced inflammation. J Immunol. 176:6245-6253.

288. Celli J, Tsolis RM. 2015. Bacteria, the endoplasmic reticulum and the unfolded protein response: friends or foes? Nat Rev Microbiol. 13:71-82.

289. Joosten LA, van De Loo FA, Lubberts E, Helsen MM, Netea MG, van Der Meer JW, Dinarello CA, van Den Berg WB. 2000. An IFN-gamma-independent proinflammatory role of IL-18 in murine streptococcal cell wall arthritis. J Immunol. 165:6553-6558.

290. Jorgensen I, Lopez JP, Laufer SA, Miao EA. 2016. IL-1 $\beta$, IL-18, and eicosanoids promote neutrophil recruitment to pore-induced intracellular traps following pyroptosis. Eur J Immunol. 46:2761-2766.

291. Young EJ, Borchert M, Kretzer FL, Musher DM. 1985. Phagocytosis and killing of Brucella by human polymorphonuclear leukocytes. J Infect Dis. 151:682-690.

292. Herbst S, Schaible UE, Schneider BE. 2011. Interferon gamma activated macrophages kill mycobacteria by nitric oxide induced apoptosis. PloS one 6:e19105.

293. Pei J, Turse JE, Wu Q, Ficht TA. 2006. Brucella abortus rough mutants induce macrophage oncosis that requires bacterial protein synthesis and direct interaction with the macrophage. Infect Immun. 74:2667-2675.

294. Meunier E, Dick MS, Dreier RF, Schürmann N, Broz DK, Warming S, RooseGirma M, Bumann D, Kayagaki N, Takeda K. 2014. Caspase-11 activation requires lysis of pathogen-containing vacuoles by IFN-induced GTPases. Nature. 509:366.

295. Pilla DM, Hagar JA, Haldar AK, Mason AK, Degrandi D, Pfeffer K, Ernst RK, Yamamoto M, Miao EA, Coers J. 2014. Guanylate binding proteins promote caspase-11-dependent pyroptosis in response to cytoplasmic LPS. Proc Natl Acad Sci. 111:6046-6051.

296. Santos JC, Dick MS, Lagrange B, Degrandi D, Pfeffer K, Yamamoto M, Meunier E, Pelczar P, Henry T, Broz P. 2018. LPS targets host guanylate-binding proteins to the bacterial outer membrane for non-canonical inflammasome activation. EMBO J. 37:e98089. 
297. Chapman R, Phillips J, Hipkin R, Curran A, Lundell D, Fine J. 2009. CXCR2 antagonists for the treatment of pulmonary disease. Pharmacol Ther. 121:55-68.

298. Singh S, Sadanandam A, Nannuru KC, Varney ML, Mayer-Ezell R, Bond R, Singh RK. 2009. Small-molecule antagonists for CXCR2 and CXCR1 inhibit human melanoma growth by decreasing tumor cell proliferation, survival, and angiogenesis. Clin Cancer Res. 15:2380-2386.

299. Bizzarri C, Beccari AR, Bertini R, Cavicchia MR, Giorgini S, Allegretti M. 2006. ELR+ CXC chemokines and their receptors (CXC chemokine receptor 1 and CXC chemokine receptor 2) as new therapeutic targets. Pharmacol Ther. 112:139-149.

300. Chapman RW, Minnicozzi M, Celly CS, Phillips JE, Kung TT, Hipkin RW, Fan X, Rindgen D, Deno G, Bond R. 2007. A novel, orally active CXCR1/2 receptor antagonist, Sch527123, inhibits neutrophil recruitment, mucus production, and goblet cell hyperplasia in animal models of pulmonary inflammation. J Pharmacol Exp Ther. 322:486-493.

301. Walters I, Austin C, Austin R, Bonnert R, Cage P, Christie M, Ebden M, Gardiner S, Grahames C, Hill S. 2008. Evaluation of a series of bicyclic CXCR2 antagonists. Bioorg Med Chem Lett. 18:798-803.

302. Kay NE, Bumol T, Douglas S. 1980. Effects of 2-deoxy-D-glucose on human monocyte metabolism and function. J Reticuloendothelial Soc. 28:367-379.

303. Cramer T, Yamanishi Y, Clausen BE, Förster I, Pawlinski R, Mackman N, Haase VH, Jaenisch R, Corr M, Nizet V, Firestein GS, Gerber H-P, Ferrara N, Johnson RS. 2003. HIF-1a is essential for myeloid cell-mediated inflammation. Cell. 112:645-657.

304. Boxer LA, Baehner RL, Davis J. 1977. The effect of 2-deoxyglucose on guinea pig polymorphonuclear leukocyte phagocytosis. J Cell Physiol. 91:89-102.

305. Dwarakanath B, Jain V. 2009. Targeting glucose metabolism with 2-deoxy-Dglucose for improving cancer therapy. Future oncol. 5:581-585.

306. Liu N, Wang L, Sun C, Yang L, Tang B, Sun W, Peng Q. 2015. Macrophage activation induced by Brucella DNA suppresses bacterial intracellular replication via enhancing NO production. Microb Pathog. 89:177-183.

307. Burt HM, Tsallas A, Gilchrist S, Liang LS. 2009. Intra-articular drug delivery systems: overcoming the shortcomings of joint disease therapy. Expert Opin Drug Deliv. 6:17-26. 
308. Butoescu N, Jordan O, Doelker E. 2009. Intra-articular drug delivery systems for the treatment of rheumatic diseases: A review of the factors influencing their performance. Eur J Pharm Biopharm. 73:205-218.

309. Choulaki C, Papadaki G, Repa A, Kampouraki E, Kambas K, Ritis K, Bertsias G, Boumpas DT, Sidiropoulos P. 2015. Enhanced activity of NLRP3 inflammasome in peripheral blood cells of patients with active rheumatoid arthritis. Arthritis Res Ther. 17:257.

310. Guo H, Callaway JB, Ting JP. 2015. Inflammasomes: mechanism of action, role in disease, and therapeutics. Nat Med. 21:677.

311. Robertson S, Martinez GJ, Payet CA, Barraclough JY, Celermajer DS, Bursill C, Patel S. 2016. Colchicine therapy in acute coronary syndrome patients acts on caspase-1 to suppress NLRP3 inflammasome monocyte activation. Clin Sci (Lond). 130:1237-1246. 


\section{VITA}

Carolyn A. Lacey was born to John and Nancy Lacey on March $11^{\text {th }}, 1991$ in Creve Coeur Missouri. She was raised in Troy, Missouri, and is the younger sister of Lauren Lacey and the older sister to Patrick Lacey. Carolyn graduated from Troy Buchanan High School in 2009 and did her undergraduate work at the University of Missouri where she received her Bachelor of Science in Biochemistry in 2013. She was accepted to the Molecular Pathogenesis and Therapeutics graduate program at the University of Missouri in 2013 where she trained under the supervision of Dr. Jerod A. Skyberg, who at the time was a newly appointed faculty member. Carolyn then received her PhD in Microbiology in May 2018. 